\title{
Asymptotic Analysis of a Storage Allocation Model with Finite Capacity: Joint Distribution
}

\author{
Eunju Sohn ${ }^{1}$ and Charles Knessl ${ }^{2}$ \\ ${ }^{1}$ Department of Science and Mathematics, Columbia College Chicago, 623 South Wabash Avenue, Chicago, IL 60605, USA \\ ${ }^{2}$ Department of Mathematics, Statistics and Computer Science, University of Illinois at Chicago, Chicago, IL 60607-7045, USA \\ Correspondence should be addressed to Eunju Sohn; esohn@colum.edu
}

Received 7 August 2015; Accepted 26 January 2016

Academic Editor: Hsien-Chung Wu

Copyright ( 2016 E. Sohn and C. Knessl. This is an open access article distributed under the Creative Commons Attribution License, which permits unrestricted use, distribution, and reproduction in any medium, provided the original work is properly cited.

We consider a storage allocation model with a finite number of storage spaces. There are $m$ primary spaces and $R$ secondary spaces. All of them are numbered and ranked. Customers arrive according to a Poisson process and occupy a space for an exponentially distributed time period, and a new arrival takes the lowest ranked available space. We let $N_{1}$ and $N_{2}$ denote the numbers of occupied primary and secondary spaces and study the joint distribution $\operatorname{Prob}\left[N_{1}=k, N_{2}=r\right]$ in the steady state. The joint process $\left(N_{1}, N_{2}\right)$ behaves as a random walk in a lattice rectangle. We study the problem asymptotically as the Poisson arrival rate $\lambda$ becomes large, and the storage capacities $m$ and $R$ are scaled to be commensurably large. We use a singular perturbation analysis to approximate the forward Kolmogorov equation(s) satisfied by the joint distribution.

\section{Introduction}

We consider the following storage allocation model. There are $m$ primary and $R$ secondary storage spaces. The primary spaces are numbered $\{1,2, \ldots, m\}$ and the secondary ones are numbered $\{m+1, m+2, \ldots, m+R\}$. Customers arrive according to a Poisson process of rate $\lambda$, and each customer occupies a storage space for an exponentially distributed amount of time, with the mean occupation time $1 / \mu$. A new arrival takes the lowest ranked available space. If all $m+R$ spaces are filled, then a new arrival is turned away and lost. The policy of taking the lowest ranked space is called "first-fit allocation."

We can consider the storage spaces as parking spaces of a restaurant. The primary spaces are in a lot right next to the restaurant, and the secondary spaces are located somewhere further away from the restaurant. Lower ranked spaces will be closer to the restaurant so it is natural for a customer to use the first-fit policy. Since spaces are occupied and emptied at random times, this model is called a dynamic storage allocation model. Design and analysis of algorithms for dynamic storage allocation are a fundamental part of computer science [1]. In such applications we can consider the customers as records, files, or lists and the storage device as a memory device. As time evolves, items are inserted and deleted, and the storage device, which is a linear array of "cells," will have regions of occupied cells alternating with interior holes. This is referred to as memory fragmentation in computers, and collapsing the holes corresponds to running a defragmentation program.

In the language of queueing theory, the model with finite secondary storage spaces can be called the $M / M /(m+$ $R) /(m+R)$ queue (the Erlang loss model) with ranked servers. The main contribution here is to study the effects of the finite storage capacity, for systems with a large number of both primary and secondary storage spaces and a commensurably large traffic intensity, which we denote by $\rho=\lambda / \mu$. Thus we study the model asymptotically for $\rho \rightarrow \infty$ with $m, R=\Theta(\rho)$.

We let $N_{1}$ and $N_{2}$ be the numbers of occupied primary and secondary spaces, and we will focus on the joint distribution of $N_{1}$ and $N_{2}$, in the steady state. The distributions of both $N_{1}$ and $N_{1}+N_{2}$ are readily computed, as these processes behave as Erlang loss models, with $m$ and $m+R$ servers, respectively. Thus their steady state distributions are 
truncated Poisson distributions. However, the distribution of the number $\mathrm{N}_{2}$ of occupied secondary spaces is much more complicated, as is the joint distribution $\operatorname{Prob}\left[N_{1}=k, N_{2}=r\right]$.

We focus here on only the steady state distribution but comment that the transient behavior of the standard Erlang loss model can be analyzed by singular perturbation methods of the type employed here (see [2]). Thus we believe that, with significant additional effort, the transient behavior of the joint process $\left(N_{1}, N_{2}\right)$ could also be ultimately analyzed.

There has been much past work on the model with an infinite (secondary) storage capacity $(R=\infty)$ since Kosten [3]. Various aspects of the solution were also studied in [4-7], but the solutions are in a complicated form, which is difficult to evaluate asymptotically for $\rho \rightarrow \infty$, due to the presence of an alternating sum. We derived the joint steady state distribution of the $\left(N_{1}, N_{2}\right)$ process in [8] using a discrete version of the classic method of separation of variables. We obtained the solution as a contour integral that involves certain polynomials related to hypergeometric functions. Such representations enabled us to obtain a complete set of asymptotic results including the joint distribution $\operatorname{Prob}\left[N_{1}=\right.$ $\left.k, N_{2}=r\right]$, for $R=\infty$ [9-12].

The solution of the finite capacity model with $R<\infty$ seems more complicated than the solution of the model with $R=\infty$. But we will show here that a singular perturbation analysis is again fruitful, and we will obtain a complete set of asymptotic results for $\pi(k, r)$, which depends also parametrically on $\rho$, and the numbers $m$ and $R$ of primary and secondary storage spaces. Most of the time we will scale all of $k, r, m$, and $R$ to be of the same order as the traffic intensity $\rho$. We will focus on understanding the effects of the finiteness of the secondary storage capacity $R$.

The remainder of the paper is organized as follows. In Section 2 we state the basic equations and briefly describe their forthcoming analysis. In Section 3 we summarize all of the main results, and the joint distribution will have different asymptotic expansions in three main regions of the state space, which is the lattice rectangle $\{(k, r): 0 \leqslant k \leqslant$ $m, 0 \leqslant r \leqslant R\}$. Moreover, there are also various boundary, corner, and transition curves where different expansions will be needed. In Section 4 we derive the asymptotics of the joint distribution in the three main regions, while in Sections 57 we treat the boundary, corner, and transition ranges. In Section 8 we will do some numerical comparisons to test the accuracy and robustness of our asymptotic results. Some discussion of our results also appears in Section 8. Since the analysis is quite technical, we have written this paper so that the derivations in Sections 4-7 can be omitted upon a first (and perhaps even later) reading(s).

\section{Statement of the Problem}

We consider a system with $m$ primary and $R$ secondary storage spaces (or servers). The primary spaces are ranked and numbered $1,2, \ldots, m$ while the secondary spaces are numbered $m+1, m+2, \ldots, m+R$. Customers arrive according to a Poisson process with rate parameter $\lambda$ and a new arrival takes the lowest ranked available space, if possible a primary one. If all $m+R$ spaces are occupied further arrivals are turned away and lost. All of the storage spaces are identical and a customer occupies a space for an exponentially distributed amount of time, with the mean occupation (or service) time being $1 / \mu$. We then let $N_{1}$ and $N_{2}$ be the numbers of occupied primary and secondary spaces, respectively. We also introduce a dimensionless parameter

$$
\rho=\frac{\lambda}{\mu}
$$

to denote the traffic intensity.

The joint process $\left(N_{1}, N_{2}\right)$ corresponds to a continuous time random walk in a lattice rectangle. Figure 1 indicates transition rates. The steady state distribution

$$
\begin{aligned}
& \pi(k, r)=\pi(k, r ; \rho, m, R)=\lim _{t \rightarrow \infty} \operatorname{Prob}\left[N_{1}(t)\right. \\
& \left.=k, N_{2}(t)=r \mid N_{1}(0)=k^{(0)}, N_{2}(0)=r^{(0)}\right]
\end{aligned}
$$

is independent of the initial values $N_{1}(0)$ and $N_{2}(0)$ and satisfies the following balance equations:

$$
\begin{aligned}
& (\rho+k+r) \pi(k, r)=(r+1) \pi(k, r+1) \\
& +(k+1) \pi(k+1, r) \\
& +\rho \pi(k-1, r), \\
& 1 \leqslant k \leqslant m-1,0 \leqslant r \leqslant R-1, \\
& (\rho+m+r) \pi(m, r)=(r+1) \pi(m, r+1) \\
& +\rho \pi(m-1, r) \\
& +\rho \pi(m, r-1) \text {, } \\
& 1 \leqslant r \leqslant R-1, \\
& (\rho+k+R) \pi(k, R)=(k+1) \pi(k+1, R) \\
& +\rho \pi(k-1, R), \\
& 1 \leqslant k \leqslant m-1, \\
& (\rho+r) \pi(0, r)=(r+1) \pi(0, r+1)+\pi(1, r), \\
& 0 \leqslant r \leqslant R-1 \text {, } \\
& (\rho+R) \pi(0, R)=\pi(1, R), \\
& (\rho+m) \pi(m, 0)=\rho \pi(m-1,0)+\pi(m, 1), \\
& (m+R) \pi(m, R)=\rho \pi(m, R-1)+\rho \pi(m-1, R) .
\end{aligned}
$$

The main balance equation (3) applies in the interior of the lattice rectangle and along the boundary $r=0$, (4)(6) correspond to boundary conditions along three of the four boundaries of the rectangle, and (7)-(9) are corner conditions. Also, (6) applies at $r=0$ so the corner condition at $(0,0)$ is $\rho \pi(0,0)=\pi(0,1)+\pi(1,0)$. We also have the normalization condition

$$
\sum_{k=0}^{m} \sum_{r=0}^{R} \pi(k, r)=1 .
$$




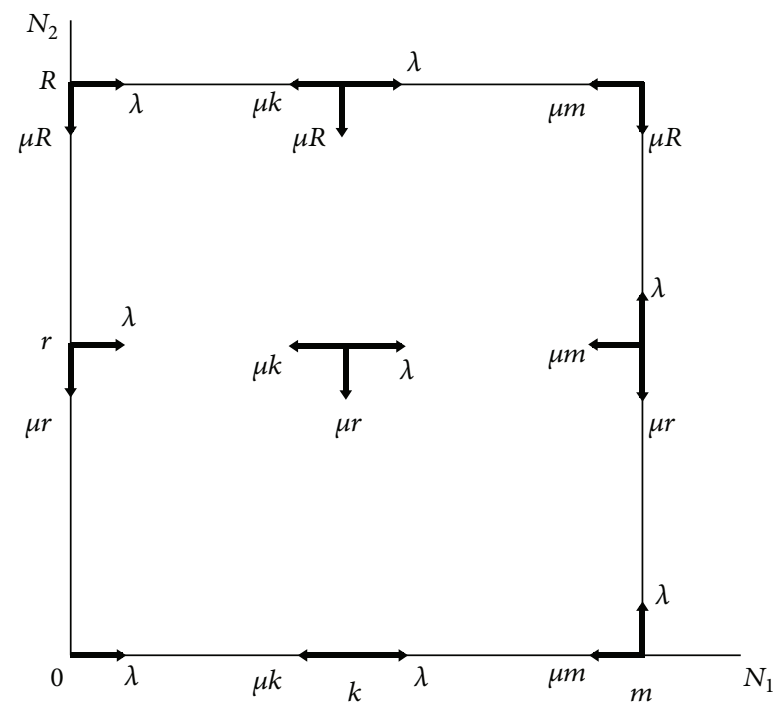

FIGURE 1: Steady state transition rates.

The process $N_{1}$ by itself behaves precisely as the Erlang loss model $(M / M / m / m$ queue with $m$ servers). This is well known to have, in the steady state, a truncated Poisson distribution; hence

$$
\sum_{r=0}^{R} \pi(k, r)=\frac{\rho^{k} e^{-\rho} / k !}{\sum_{k=0}^{m} \rho^{k} e^{-\rho} / k !}, \quad 0 \leqslant k \leqslant m .
$$

The total number, $N_{1}+N_{2}$, of occupied servers also follows a truncated Poisson distribution. Therefore,

$$
\begin{aligned}
& \sum_{k+r=L} \pi(k, r)=\sum_{k=\max \{0, L-R\}}^{\min \{m, L\}} \pi(k, L-k) \\
& =\frac{\rho^{L} e^{-\rho} / L !}{\sum_{\ell=0}^{m+R} \rho^{\ell} e^{-\rho} / \ell !}, \quad 0 \leqslant L \leqslant m+R \text {. }
\end{aligned}
$$

We recently obtained in [13] explicit expressions for the joint distribution $\pi(k, r)$, but they are not very insightful due to their complexity. Thus we study the problem asymptotically, for $\rho \rightarrow \infty$ with $m, R=O(\rho)$. This means that there are many arrivals but the numbers of storage spaces, both primary and secondary ones, are commensurately large. Note that if $\rho \rightarrow \infty$ with $m, R=O(1)$ then the probability distribution $\pi(k, r)$ would concentrate on a single lattice point, with $\pi(k, r) \rightarrow \delta_{k, m} \delta_{r, R}$ as $\rho \rightarrow \infty$. Here $\delta_{k, m}=0$, $k \neq m$, and $\delta_{k, m}=1, k=m$. Thus this limit would not be particularly interesting. There are, however, certain cases where either $m$ or $R$ is large but $o(\rho)$, that should lead to interesting results, but we do not analyze them here.

We next introduce the parameters

$$
\begin{aligned}
& X_{0}=\frac{m}{\rho}, \\
& Y_{0}=\frac{R}{\rho}
\end{aligned}
$$

and in the present limit we have $0<X_{0}, Y_{0}<\infty$, where we view $X_{0}$ and $Y_{0}$ as fixed as $\rho \rightarrow \infty$. We may then view the process $\left(N_{1}, N_{2}\right)$ on a "coarse" spatial scale, with

$$
\begin{aligned}
& X=\frac{k}{\rho}, \quad 0 \leqslant X \leqslant X_{0}, \\
& Y=\frac{r}{\rho}, \quad 0 \leqslant Y \leqslant Y_{0} .
\end{aligned}
$$

On the $(X, Y)$ scale the random walk takes small steps (= $1 / \rho)$ and the state space may be approximately viewed as the continuous rectangle

$$
\left\{(X, Y): 0 \leqslant X \leqslant X_{0}, 0 \leqslant Y \leqslant Y_{0}\right\}
$$

Setting

$$
\begin{aligned}
\pi(k, r) & =\pi(k, r ; \rho, m, R)=P(X, Y) \\
& =P\left(X, Y ; \rho, X_{0}, Y_{0}\right)
\end{aligned}
$$

the main balance equation (3) becomes

$$
\begin{aligned}
(1+X+Y) P(X, Y)= & \left(Y+\rho^{-1}\right) P\left(X, Y+\rho^{-1}\right) \\
& +\left(X+\rho^{-1}\right) P\left(X+\rho^{-1}, Y\right) \\
& +P\left(X-\rho^{-1}, Y\right)
\end{aligned}
$$

which is a difference equation with small differences, of order $O\left(\rho^{-1}\right)$. The boundary condition along $k=m$ in (4) may be replaced by the "artificial boundary condition"

$$
(m+1) \pi(m+1, r)=\rho \pi(m, r-1), \quad 1 \leqslant r \leqslant R-1 .
$$

The above is obtained by requiring that (3) holds also at $k=m$ and comparing this to (4). Introducing $\pi(m+1, r)$ simplifies some of the calculations, but this quantity has no physical meaning.

The asymptotic structure of the joint distribution will be very different for four main regions in the $\left(X_{0}, Y_{0}\right)$ parameter space. We call these regions $\mathscr{R}_{1}-\mathscr{R}_{4}$ and they are sketched in Figure 2. They are defined by the inequalities

$$
\begin{aligned}
\mathscr{R}_{1} & =\left\{\left(X_{0}, Y_{0}\right): X_{0}>1, Y_{0}>0\right\}, \\
\mathscr{R}_{2} & =\left\{\left(X_{0}, Y_{0}\right): 0<X_{0}<1, Y_{0}+X_{0}>1\right\}, \\
\mathscr{R}_{3} & =\left\{\left(X_{0}, Y_{0}\right): 0<X_{0}<1, \sqrt{X_{0}}-X_{0}<Y_{0}<1\right. \\
& \left.-X_{0}\right\}, \\
\mathscr{R}_{4} & =\left\{\left(X_{0}, Y_{0}\right): 0<X_{0}<1,0<Y_{0}<\sqrt{X_{0}}-X_{0}\right\} .
\end{aligned}
$$




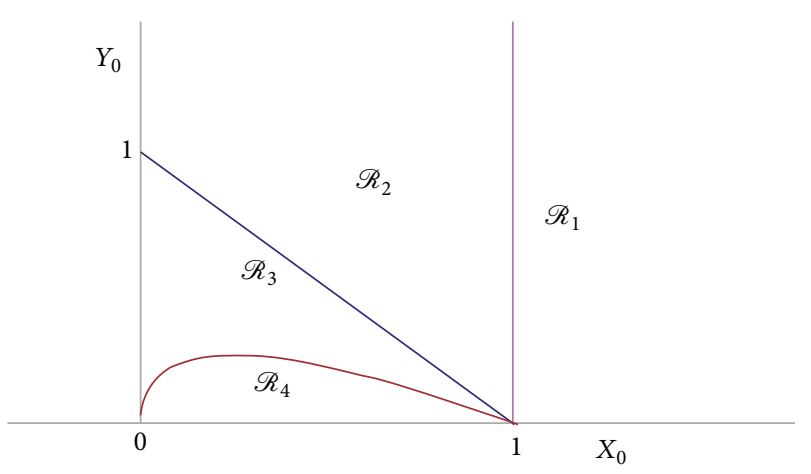

FIgURE 2: Four regions of the $\left(X_{0}, Y_{0}\right)$ parameter space.

It will also prove useful to define as follows the curves that separate these four regions:

$$
\begin{aligned}
& \mathscr{R}_{1} \cap \mathscr{R}_{2}=\left\{\left(X_{0}, Y_{0}\right): X_{0}=1, Y_{0}>0\right\}, \\
& \mathscr{R}_{2} \cap \mathscr{R}_{3}=\left\{\left(X_{0}, Y_{0}\right): X_{0}+Y_{0}=1,0<X_{0}<1\right\}, \\
& \mathscr{R}_{3} \cap \mathscr{R}_{4} \\
& \quad=\left\{\left(X_{0}, Y_{0}\right): X_{0}+Y_{0}=\sqrt{X_{0}}, 0<X_{0}<1\right\} .
\end{aligned}
$$

Note that the union of all the sets in (19)-(25) is the entire open quarter plane in parameter space. We purposefully exclude the coordinate axes $X_{0}=0$ and $Y_{0}=0$, as they would require entirely different asymptotic analyses. The separating curves in (23)-(25) will also require separate analyses, and we will obtain results that apply not only along the curves but also in small neighborhoods of these curves, which will be defined precisely later. This will produce results that asymptotically match to those in the main regions.

The presence of the different regions can be explained intuitively. If $X_{0}>1(m>\rho)$ there are enough primary spaces to service all storage requests and the secondary spaces will generally not be needed. If $X_{0}<1$ but $X_{0}+Y_{0}>$ $1(m+R>\rho)$ the primary spaces are insufficient but the total number of spaces is adequate. Then we might expect that typically all $m$ primary spaces and about $\rho-m(<R)$ secondary spaces will be occupied. If $X_{0}+Y_{0}<1$ then typically all primary and secondary spaces will be occupied. Then we might expect $\pi(k, r)$ to be concentrated near $k=m$, $r=R$. The further splitting of $X_{0}+Y_{0}<1$ into the regions $\mathscr{R}_{3}$ and $\mathscr{R}_{4}$ is difficult to explain intuitively in terms of the basic model, but we will explain this dichotomy via our asymptotic analysis. We also note that the asymptotic behavior of the distribution in (11) undergoes a transition when $m / \rho$ passes through 1 , while (12) undergoes an analogous transition when $(m+R) / \rho$ passes through 1 . However, neither (11) nor (12) undergoes a transition along $\mathscr{R}_{3} \cap \mathscr{R}_{4}$. In the analysis that follows we will also need to, for each region of parameter space, separately analyze several different regions of the state space, which corresponds to the rectangle $0 \leqslant X \leqslant X_{0}$, $0 \leqslant Y \leqslant Y_{0}$ on the coarse spatial scale. It will sometimes prove necessary to analyze boundary and corner regions where the discrete nature of the model must be considered.

\section{Summary of Results}

In the analysis it proves sometimes useful to use the variables $(n, p)$ where

$$
\begin{aligned}
& n=m-k, \\
& p=R-r,
\end{aligned}
$$

so that $n$ (resp., $p$ ) measures the number of unoccupied primary (resp., secondary) spaces. Then we also let

$$
\pi(k, r)=\pi(m-n, R-p)=Q(n, p)
$$

so that $Q(0,0)$ corresponds to the probability that all of the storage spaces are full. In (27) we did not indicate the dependence of $\pi$ and $Q$ on the parameters $\rho, m$, and $R$.

We begin by giving asymptotic results for $Q(0,0)$.

Proposition 1. For $\rho \rightarrow \infty$ and fixed $X_{0}, Y_{0}$ one has

$$
\begin{aligned}
& \pi(m, R)=Q(0,0) \sim 1-X_{0}-Y_{0}, \\
& \left(X_{0}, Y_{0}\right) \in \mathscr{R}_{3} \cup \mathscr{R}_{4}, \\
& Q(0,0) \sim \frac{1}{\sqrt{\rho}} \frac{e^{-\gamma^{2} / 2}}{\int_{-\infty}^{\gamma} e^{-u^{2} / 2} d u}, \\
& X_{0}+Y_{0}=1+\frac{\gamma}{\sqrt{\rho}}, \quad \gamma=O(1)\left(\mathscr{R}_{2} \cap \mathscr{R}_{3}\right),
\end{aligned}
$$

$Q(0,0)$

$$
\begin{array}{r}
\sim \frac{1}{\sqrt{\rho}} \frac{1}{\sqrt{2 \pi\left(X_{0}+Y_{0}\right)}} e^{\rho\left[X_{0}+Y_{0}-1-\left(X_{0}+Y_{0}\right) \log \left(X_{0}+Y_{0}\right)\right]}, \\
\left(X_{0}, Y_{0}\right) \in \mathscr{R}_{1} \cup \mathscr{R}_{2} .
\end{array}
$$

Here and throughout the paper, we use the convention that $\mathscr{R}_{1} \cup \mathscr{R}_{2}$ corresponds to the union of the open sets $\mathscr{R}_{1}$ and $\mathscr{R}_{2}$ and also the separating curve $\mathscr{R}_{1} \cap \mathscr{R}_{2}$ (cf. (23)). Similar comments apply for $\mathscr{R}_{3} \cup \mathscr{R}_{4}$ and $\mathscr{R}_{2} \cup \mathscr{R}_{3}$. We refer to the asymptotic limit in (29) as corresponding to $\mathscr{R}_{2} \cap \mathscr{R}_{3}$, where we now give the precise scaling, $X_{0}+Y_{0}-1=O\left(\rho^{-1 / 2}\right)$, that applies near the separating curve in (24). The results in (28)-(30) will follow from our asymptotic analysis of the joint distribution, but we note that these also follow easily from (12), by setting $N=m+R$ and expanding the result for $\rho \rightarrow \infty$ and different ranges of $Z_{0}=X_{0}+Y_{0}$ (thus $Z_{0}=N / \rho$ ).

It will prove convenient to express some of our results in terms of the three constants $C, C_{0}$, and $C_{1}$; these depend only on the parameters $\rho, X_{0}$, and $Y_{0}$. We summarize below the leading order asymptotics of these constants.

Proposition 2. Define the constants $C, C_{1}$, and $C_{0}$ by the relations

$$
\begin{aligned}
& C \sim \rho^{-1 / 2}, \quad\left(X_{0}, Y_{0}\right) \in \mathscr{R}_{1} \cup \mathscr{R}_{2}, \\
& C \sim \sqrt{\frac{2 \pi}{\rho}}\left[\int_{-\infty}^{\gamma} e^{-u^{2} / 2} d u\right]^{-1}, \quad\left(X_{0}, Y_{0}\right) \in \mathscr{R}_{2} \cap \mathscr{R}_{3},
\end{aligned}
$$




$$
\begin{aligned}
& C \sim \sqrt{2 \pi\left(X_{0}+Y_{0}\right)}\left(1-X_{0}-Y_{0}\right) \\
& \cdot e^{\rho\left[1-X_{0}-Y_{0}+\left(X_{0}+Y_{0}\right) \log \left(X_{0}+Y_{0}\right)\right]}, \quad\left(X_{0}, Y_{0}\right) \in \mathscr{R}_{3}, \\
& C_{1} \sim \rho^{-2 / 3}, \quad\left(X_{0}, Y_{0}\right) \in \mathscr{R}_{2}, \\
& C_{1} \sim \rho^{-2 / 3} \frac{\sqrt{2 \pi}}{\int_{-\infty}^{\gamma} e^{-u^{2} / 2} d u}, \quad\left(X_{0}, Y_{0}\right) \in \mathscr{R}_{2} \cap \mathscr{R}_{3} \text {, } \\
& C_{1} \sim \rho^{1 / 6} \sqrt{2 \pi\left(X_{0}+Y_{0}\right)}\left(1-X_{0}-Y_{0}\right) \\
& \cdot e^{\rho\left[1-X_{0}-Y_{0}+\left(X_{0}+Y_{0}\right) \log \left(X_{0}+Y_{0}\right)\right]}, \quad\left(X_{0}, Y_{0}\right) \in \mathscr{R}_{3}, \\
& C_{1} \sim \rho^{-1 / 6}\left(1-X_{0}-Y_{0}\right) \\
& \cdot e^{-\rho \Phi\left(X_{0}, Y_{0}\right)} \sqrt{2 \pi} X_{0}^{1 / 4} \int_{r_{0}}^{\infty} e^{\delta_{1} u} A i(u) d u, \\
& X_{0}+Y_{0}-\sqrt{X_{0}}=\rho^{-1 / 3} \delta_{*}=O\left(\rho^{-1 / 3}\right) \\
& \left(\mathscr{R}_{3} \cap \mathscr{R}_{4}\right) \text {, } \\
& \delta_{1}=\frac{\delta_{*}}{X_{0}^{1 / 6}\left(1-\sqrt{X_{0}}\right)^{1 / 3}}, \\
& \Phi\left(X_{0}, Y_{0}\right)=\left(Y_{0}+W_{0}\right) \log \left(Y_{0}+W_{0}\right)-Y_{0} \log Y_{0} \\
& -\frac{W_{0}}{2} \log W_{0}-\frac{X_{0}}{2} \log X_{0}+\sqrt{X_{0}}-1, \\
& W_{0}=\left(1-\sqrt{X_{0}}\right)^{2}
\end{aligned}
$$

where $A i(\cdot)$ is the Airy function and $r_{0}$ its maximal root

$$
\begin{aligned}
r_{0}= & \max \{z: A i(z)=0\}=-2.3381 \cdots \\
C_{1} & \sim \rho^{-5 / 6} \sqrt{2 \pi}\left(1-X_{0}-Y_{0}\right) A i^{\prime}\left(r_{0}\right) \\
& \cdot e^{-\rho \Phi\left(X_{0}, Y_{0}\right)} e^{-\rho^{1 / 3} \Phi_{*}\left(Y_{0}\right)} \\
& \cdot \frac{\sqrt{Y_{0}} X_{0}^{1 / 3}\left(1-\sqrt{X_{0}}\right)^{2 / 3}}{\left(\sqrt{X_{0}}-X_{0}-Y_{0}\right)^{2} \sqrt{W_{0}+Y_{0}}}\left(\frac{Y_{0}+W_{0}}{1-\sqrt{X_{0}}}\right)^{1 / 2 \sqrt{X_{0}}}, \\
\Phi_{*} & \left(Y_{0}\right)=-r_{0}\left(1-\sqrt{X_{0}}\right)^{2 / 3} X_{0}^{-1 / 6} \log \left(\frac{Y_{0}+W_{0}}{1-\sqrt{X_{0}}}\right), \\
C_{0} & \sim \rho^{-1 / 2}\left(1-X_{0}, Y_{0}\right) \in \mathscr{R}_{4}, \\
C_{0} & \sim \rho^{-1} \frac{e^{-\gamma^{2} / 2}}{\int_{-\infty}^{\gamma} e^{-u^{2} / 2} d u}, \quad\left(X_{0}, Y_{0}\right) \in \mathscr{R}_{3} \cup \mathscr{R}_{4}, \\
C_{0} & \sim \rho^{-1}\left[2 \pi\left(X_{0}+Y_{0}\right)\right]^{-1 / 2}, \mathscr{R}_{2} \cap \mathscr{R}_{3}, \\
& \cdot e^{\rho\left[X_{0}+Y_{0}-1-\left(X_{0}+Y_{0}\right) \log \left(X_{0}+Y_{0}\right)\right]},
\end{aligned}
$$

$$
\left(X_{0}, Y_{0}\right) \in \mathscr{R}_{1} \cup \mathscr{R}_{2}
$$

We note that the relation $C_{0} \sim \rho^{-1 / 2} Q(0,0)$ holds for all cases of the parameters. In (38) we have thus defined the precise scaling near the separating curve $\mathscr{R}_{3} \cap \mathscr{R}_{4}$, as $Y_{0}=$ $\sqrt{X_{0}}-X_{0}+O\left(\rho^{-1 / 3}\right)$. Note that $C$ is not defined for region $\mathscr{R}_{4}$ while $C_{1}$ is not defined for $\mathscr{R}_{1}$, as then the corresponding constant will play no role in the analysis. We conclude by giving the precise scaling for $\mathscr{R}_{1} \cap \mathscr{R}_{2}$ (near $X_{0}=1$ ), which will be

$$
X_{0}=1+\frac{\beta}{\sqrt{\rho}}=1+O\left(\rho^{-1 / 2}\right) \text {. }
$$

Note that (46) can be predicted from the marginal distribution in (11), as the sum in the denominator undergoes a transition for $m=\rho+O(\sqrt{\rho})$, which is the same scaling as in (46).

3.1. Joint Distribution and Its Limits. Now we consider the joint distribution; $\pi(k, r)=\pi(k, r ; \rho, m, R)=P(X, Y)=$ $P\left(X, Y ; \rho, X_{0}, Y_{0}\right)$ for $\rho \rightarrow \infty$. We recall that $X_{0}=m / \rho$ and $Y_{0}=R / \rho$ are the scaled numbers of primary and secondary spaces. The state space of the random walk is the lattice rectangle in Figure 1, and on the coarse $(X, Y)$ spatial scale this can be viewed as the continuous rectangle $\{(X, Y): 0 \leqslant X \leqslant$ $\left.X_{0}, 0 \leqslant Y \leqslant Y_{0}\right\}$. Our goal is to give a complete asymptotic description of the joint distribution for $\rho \rightarrow \infty$, including ranges of the state space where there is appreciable mass and also ranges where $\pi(k, r)$ is asymptotically small. This corresponds to the tails of the distribution and in such ranges $\pi(k, r)=P(X, Y)$ is typically exponentially small for large $\rho$. We first discuss the ranges where there is significant mass, and this will lead to certain limiting distributions, which will be very different for regions $\mathscr{R}_{1}-\mathscr{R}_{4}$ of parameter space in (19)-(25).

Proposition 3. For $\rho \rightarrow \infty$ one has the following limiting distributions:

(i) $X_{0}=m / \rho>1\left(\right.$ thus $\left.\left(X_{0}, Y_{0}\right) \in \mathscr{R}_{1}\right)$

$$
\pi(k, r) \approx \delta_{0 r} \frac{e^{-\rho} \rho^{k}}{k !}, \quad \frac{k}{\rho}=X<X_{0},
$$

which can be recast as the limit

$$
\begin{aligned}
& \sqrt{\rho} \pi\left(\rho+\sqrt{\rho} X^{\prime}, r\right) \longrightarrow \delta_{0 r} \frac{1}{\sqrt{2 \pi}} e^{-\left(X^{\prime}\right)^{2} / 2}, \\
& \quad \rho \longrightarrow \infty . \\
& \text { (ii) } 0<X_{0}<1, X_{0}+Y_{0}>1\left(\text { thus }\left(X_{0}, Y_{0}\right) \in \mathscr{R}_{2}\right) \\
& \pi(k, r) \sim \frac{1-X_{0}}{\sqrt{2 \pi \rho}} X_{0}^{n} \exp \left\{-\frac{\rho}{2}\left[Y-\left(1-X_{0}\right)\right]^{2}\right\}
\end{aligned}
$$

and this applies for $n=m-k=O(1)$ and $Y-\left(1-X_{0}\right)=$ $O\left(\rho^{-1 / 2}\right)$ (i.e., $r=\rho-m+O(\sqrt{\rho})$ ). 
(iii) $0<X_{0}+Y_{0}<1$ (thus $\left.\left(X_{0}, Y_{0}\right) \in \mathscr{R}_{3} \cup \mathscr{R}_{4}\right)$

$$
\begin{aligned}
& \pi(k, r) \sim \frac{1-X_{0}-Y_{0}}{X_{0}} \frac{1}{2 \pi i} \oint \frac{1-w}{z_{-}(w)-w}\left[z_{+}(w)\right]^{-n-1} \\
& \cdot w^{-p-1} d w \\
& z_{ \pm}(w)=\frac{1}{2 X_{0}}\left[1+X_{0}+Y_{0}-Y_{0} w\right. \\
& \left. \pm \sqrt{\left(1+X_{0}+Y_{0}-Y_{0} w\right)^{2}-4 X_{0}}\right]
\end{aligned}
$$

which holds for $n=m-k=O(1)$ and $p=R-r=O(1)$.

When $X_{0}>1$ we have $m>\rho$ so the secondary storage spaces will be rarely needed, and then $\pi(k, r)$ approximately follows the Poisson distribution in (47), which has also the Gaussian limit in (48). The results in (47) and (48) provide no information on $\pi(k, r)$ for $r \geqslant 1$, but later we will estimate precisely these probabilities. We also note that when $r=0$, (47) ceases to be valid for $k=\rho X_{0}-O(1)=m=O(1)$, for then if almost all primary spaces are full there may well be some secondary spaces also occupied, and thus $\pi(k, 0)$ may become comparable to $\pi(k, r)$ for $r \geqslant 1$, for this range of $k$. If $X_{0}<1$ and $X_{0}+Y_{0}>1$ the primary storage spaces are insufficient to meet the demand, but the total number of spaces does suffice. Then (49) shows that $m-O(1)$ primary spaces and $\rho\left(1-X_{0}\right)+O(\sqrt{\rho})=\rho-m+O(\sqrt{\rho})$ secondary spaces will tend to be occupied, with the joint distribution being a product of a geometric and a Gaussian. This also shows that, to leading order for large $\rho$, the processes $N_{1}$ and $N_{2}$ decouple. When $X_{0}+Y_{0}<1$ we have $m+R<\rho$ and the totality of storage spaces is not enough to meet the demand. Then typically all but a few spaces, both primary and secondary, will tend to be occupied, with the numbers $n$ and $p$ of available spaces following the discrete joint distribution in (50). From (50) we can easily show that

$$
\sum_{k+r=L} \pi(k, r) \sim\left(1-X_{0}-Y_{0}\right)\left(X_{0}+Y_{0}\right)^{L}
$$

so that the total number of empty spaces is geometrically distributed; this result also follows easily from the exact expression in (12). We will later see that the tail behavior of (50), for $n$ and/or $p \rightarrow \infty$, is quite different according as $\left(X_{0}, Y_{0}\right) \in \mathscr{R}_{3}$ or $\left(X_{0}, Y_{0}\right) \in \mathscr{R}_{4}$, which again will indicate that the triangle $0<X_{0}+Y_{0}<1$ in parameter space needs to be split into the two regions $\mathscr{R}_{3}$ and $\mathscr{R}_{4}$.

We next study the transitions between the three limiting results in Proposition 4.

Proposition 4. For $\rho \rightarrow \infty$ one has the limiting distributions: (i) $X_{0}-1=\beta / \sqrt{\rho}=O\left(\rho^{-1 / 2}\right), X-1=\alpha / \sqrt{\rho}$, and $Y=\Omega / \sqrt{\rho}$ (thus $k=m+\alpha \sqrt{\rho}$ and $r=\sqrt{\rho} \Omega$ )

$$
\begin{array}{r}
\pi(k, r) \sim \rho^{-1} e^{-\alpha^{2} / 4} \frac{1}{2 \pi i} \int_{-i \infty}^{i \infty} \frac{\Omega^{z-1} D_{z}(-\alpha)}{D_{z}(-\beta) D_{z-1}(-\beta)} d z, \\
-\infty<\alpha<\beta, \Omega>0,
\end{array}
$$

where $D_{z}(-\beta)$ is the parabolic cylinder function of order $Z$ and argument $-\beta$. When $\beta=0\left(X_{0}=1\right)$ the above simplifies to

$$
\pi(k, r) \sim \rho^{-1} \sqrt{\frac{2}{\pi}}(\Omega-\alpha) e^{-(\Omega-\alpha)^{2} / 2}, \quad \alpha<0, \Omega>0 .
$$

(ii) $X_{0}+Y_{0}-1=\gamma / \sqrt{\rho}=O\left(\rho^{-1 / 2}\right), n=m-k=O(1)$, and $Y_{0}-Y=\rho^{-1 / 2} \tilde{y}=O\left(\rho^{-1 / 2}\right)$

$$
\begin{aligned}
& \pi(k, r) \\
& \sim \rho^{-1 / 2}\left(1-X_{0}\right) X_{0}^{n}\left(\int_{-\infty}^{\gamma} e^{-u^{2} / 2} d u\right)^{-1} e^{-(\tilde{y}-\gamma)^{2} / 2},
\end{aligned}
$$$$
\tilde{y}>0 \text {. }
$$

As $\gamma \rightarrow+\infty$ the truncated Gaussian distribution in (55) approaches the free space Gaussian in (49), which applies for $1-Y_{0}<X_{0}<1$. For $\gamma \rightarrow-\infty$, (55) asymptotically matches to (50), when the latter is expanded for $X_{0}+Y_{0} \uparrow 1$ and simultaneously $p \rightarrow \infty$, with the product $\left(1-X_{0}-Y_{0}\right) p=$ $-\gamma \tilde{y}$ held fixed.

The complicated distribution in (53) is a necessary intermediate result since (47) and (49) do not asymptotically match. The right-hand side of (53) is of the form $\rho^{-1} \times$ (density in $(\alpha, \Omega))$, with the density having support in the quarter plane $\alpha<\beta, \Omega>0$. Thus if $m=\rho+O(\sqrt{\rho})$ there will tend to be $O(\sqrt{\rho})$ empty primary spaces and $O(\sqrt{\rho})$ full secondary spaces, with now an intricate coupling between the processes $N_{1}$ and $N_{2}$. Finally, we note that the results in items (i) and (ii) in Proposition 3 and in item (i) of Proposition 4 are independent of the secondary storage capacity $Y_{0}=R / \rho$, while item (iii) in Proposition 3 and item (ii) in Proposition 4 do depend upon $Y_{0}$.

3.2. Joint Distribution: Main Regions of State Space. The asymptotic expansion of $\pi(k, r)=P(X, Y)$ will be different for the four parameter ranges indicated in Figure 2 and also for three main regions of the state space, which we call $\mathscr{D}_{0}$, $\mathscr{D}_{+}$, and $\mathscr{D}_{-}$, and we define/discuss these below.

First consider region $\mathscr{R}_{1}$ of parameter space, so that $X_{0}>$ 1 , and define the curve

$$
\begin{aligned}
Y & =\tilde{Y}(X)=\tilde{Y}\left(X ; X_{0}, Y_{0}\right)=Y_{0}+\frac{Y_{0}}{2\left(X_{0}+Y_{0}\right)^{2}\left(X_{0}+Y_{0}-1\right)}\left\{X_{0}-\left(X_{0}+Y_{0}\right)^{2}+\left(X_{0}+Y_{0}\right)\left(X-X_{0}\right)\right. \\
& \left.+\sqrt{\left[\left(X_{0}+Y_{0}\right)^{2}+\left(X_{0}+Y_{0}\right)\left(X_{0}-X\right)-X_{0}\right]^{2}-4\left(X_{0}+Y_{0}\right)^{2}\left(X_{0}+Y_{0}-1\right)\left(X_{0}-X\right)}\right\} .
\end{aligned}
$$




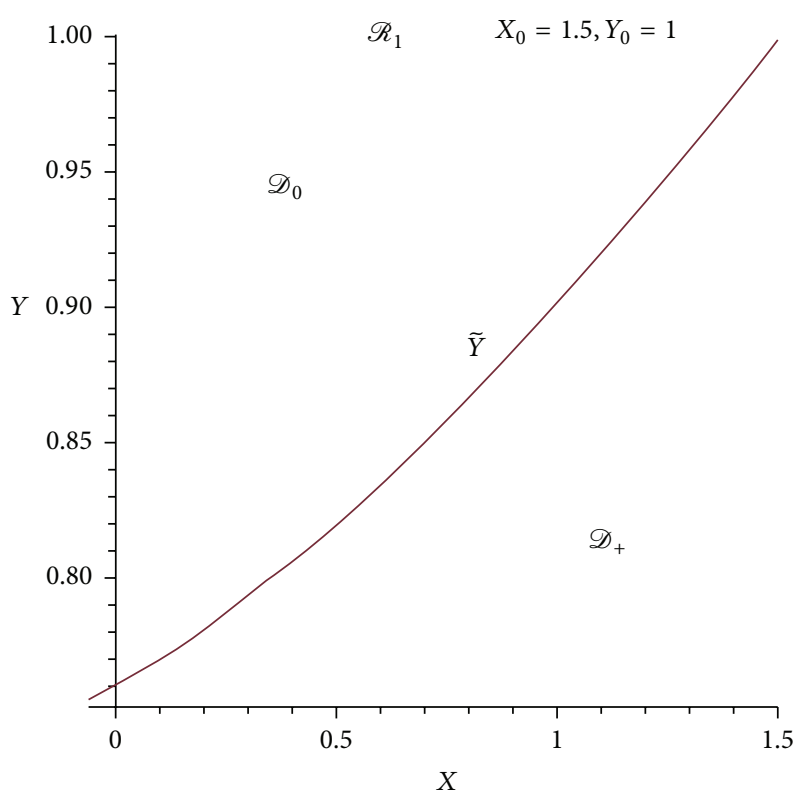

FIgURE 3: Region $\mathscr{R}_{1}$.

This curve depends on both $X_{0}$ and $Y_{0}$ and thus on both of the total numbers, $m$ and $R$, of primary and secondary storage spaces. The curve is defined for $0 \leqslant X \leqslant X_{0}$ and we have

$$
\widetilde{Y}(0)=Y_{0}\left[1-\frac{X_{0}}{\left(X_{0}+Y_{0}\right)^{2}}\right], \quad \widetilde{Y}\left(X_{0}\right)=Y_{0}
$$

For region $\mathscr{R}_{1}$ (and indeed also for $\mathscr{R}_{2}$ and $\mathscr{R}_{3}$ ) we have $\tilde{Y}(0)>0$ so that (56) connects the point $\left(0, \mathrm{Y}_{0}\left[1-X_{0} /\left(X_{0}+\right.\right.\right.$ $\left.\left.\left.Y_{0}\right)^{2}\right]\right)$ to the corner point $\left(X_{0}, Y_{0}\right)$ in the scaled state space. The curve $\widetilde{Y}(X)$ divides the state space into the two regions $\mathscr{D}_{0}$ and $\mathscr{D}_{+}$, with

$$
\begin{aligned}
& \mathscr{D}_{+}=\left\{(X, Y): 0<X \leqslant X_{0}, 0<Y<\widetilde{Y}(X)\right\}, \\
& \mathscr{D}_{0}=\left\{(X, Y): 0<X<X_{0}, \tilde{Y}(X)<Y<Y_{0}\right\} .
\end{aligned}
$$

Here we defined $\mathscr{D}_{0}$ as an open set, while $\mathscr{D}_{+}$is bounded by the four curves $X=0, Y=0, X=X_{0}$, and $Y=\tilde{Y}(X)$ and we include only the third of these $\left(X=X_{0}\right)$ as a part of $\mathscr{D}_{+}$. This is because the asymptotic expansion that will apply in the interior of $\mathscr{D}_{+}$will remain valid near $X=X_{0}$, but not near the other three bounding curves. The expansion valid in $\mathscr{D}_{0}$ will break down if either $X \approx 0, Y \approx Y_{0}$, or $Y \approx \widetilde{Y}(X)$. We sketch in Figure 3 the curve $\widetilde{Y}(X)$ and we recall that if $X_{0}>1$ most of the mass in $\pi(k, r)$ is concentrated in the range $r=0$ and $k=\rho+O(\sqrt{\rho})\left(X=1+O\left(\rho^{-1 / 2}\right)\right)$ (see Proposition 3), and this corresponds to the lower bounding curve for $\mathscr{D}_{+}$.

Proposition 5. For $\left(X_{0}, Y_{0}\right) \in \mathscr{R}_{1}\left(X_{0}>1\right)$ the asymptotic expansions of $\pi(k, r)=\pi(\rho X, \rho Y)$ are as follows: (i) $(X, Y) \in \mathscr{D}_{+}(0<Y<\tilde{Y}(X))$

$$
\begin{aligned}
& \pi(k, r) \sim C(\rho) K_{+}(X, Y) e^{\rho \Psi_{+}(X, Y)}, \\
& \text { where } C(\rho) \sim \rho^{-1 / 2},
\end{aligned}
$$

$\Psi_{+}(X, Y)=X_{0} \log X_{0}+X_{0}-1+s-2 X_{0}$

$\cdot \log \left(s+X_{0}\right)+\frac{s}{s+X_{0}}\left(1-e^{t}\right)$

$-\frac{s}{s+X_{0}}\left[s+X_{0}-1+e^{-t}\right]$

$$
\begin{aligned}
& \cdot \log \left[1+\left(s+X_{0}-1\right) e^{t}\right]+\left(e^{-t}-\frac{s}{s+X_{0}}\right) \\
& \cdot\left(1-X_{0}-s-e^{t}\right) \log \left[1-\frac{s}{s+X_{0}} e^{t}\right],
\end{aligned}
$$

where $(s, t)$ are related to $(X, Y)$ via the mapping, for $0<s<Y_{0}$,

$$
\begin{aligned}
X & =\left[1-\frac{s}{s+X_{0}} e^{t}\right]\left[1+\left(s+X_{0}-1\right) e^{-t}\right], \\
Y & =\frac{s}{s+X_{0}}\left[e^{-t}-1+X_{0}+s\right], \\
K_{+} & (X, Y)=\left[e^{-t}+s+X_{0}-1\right]^{-1 / 2} \\
& \cdot\left[e^{-t}-\frac{s}{s+X_{0}}\right]^{-1 / 2}\left|\Delta_{+}(s, t)\right|^{-1 / 2} \sqrt{\frac{X_{0}}{2 \pi}} \\
& \cdot \frac{\left[\left(s+X_{0}\right)^{2}-X_{0}\right]^{3 / 2}}{\left(s+X_{0}\right)^{3}},
\end{aligned}
$$

where $\Delta_{+}$is the Jacobian associated with (62); that is,

$$
\begin{aligned}
& \Delta_{+}(s, t)=X_{t} Y_{s}-X_{s} Y_{t}=\frac{1}{1-E}\left[D(E-D) e^{t}\right. \\
& -2 D(1-D)-(D-E)^{2} e^{-t} \\
& \left.+(E+D-2 E D) e^{-2 t}\right], \\
& D=\frac{s}{s+X_{0}}=1-\frac{X_{0}}{s+X_{0}}, E=1-X_{0}-s .
\end{aligned}
$$

(ii) $(X, Y) \in \mathscr{D}_{0}\left(\tilde{Y}(X)<Y<Y_{0}\right)$

$$
\pi(k, r) \sim C_{0}(\rho) K(X, Y) e^{\rho \Psi(X, Y)},
$$


where $C_{0}(\rho)$ is given by (45) for region $\mathscr{R}_{1}$

$$
\begin{aligned}
& \Psi(X, Y)=A\left(1-e^{\tau}\right)+X_{0} \log (1-A)+Y_{0} \\
& \cdot \log (1-B)-X \log \left(1-A e^{\tau}\right)-Y \log \left(1-B e^{\tau}\right) \\
& =-A e^{\tau}+\left[A e^{\tau}-1+\left(e^{-\tau}-A\right)\left(1-\frac{X_{0}}{1-A}\right)\right] \\
& \quad \cdot \log \left(1-A e^{\tau}\right)+\frac{Y_{0}\left(e^{-\tau}-B\right)}{B-1} \log \left(1-B e^{\tau}\right)+A \\
& +X_{0} \log (1-A)+Y_{0} \log (1-B), \\
& B=\frac{A\left(1-A-X_{0}\right)}{(1-A)\left(A+X_{0}+Y_{0}\right)-X_{0}},
\end{aligned}
$$$$
\text { where }(A, \tau) \text { are related to }(X, Y) \text { by }
$$

$$
\begin{aligned}
X & =\left(e^{-\tau}-A\right)\left(\frac{X_{0}}{1-A}-1+e^{\tau}\right) \\
Y & =Y_{0}-\left(1-e^{-\tau}\right)\left(A+X_{0}+Y_{0}-\frac{X_{0}}{1-A}\right) \\
& =Y_{0}\left(\frac{B-e^{-\tau}}{B-1}\right)
\end{aligned}
$$

where $A \in\left(A_{\min }, A_{\max }\right)$ with

$$
\begin{aligned}
& A_{\text {min }}=1-\sqrt{X_{0}}, \\
& A_{\max }=\frac{1}{2}\left[1-X_{0}-Y_{0}+\sqrt{\left(X_{0}+Y_{0}+1\right)^{2}-4 X_{0}}\right],
\end{aligned}
$$

with $A \in\left(Y_{0} /\left(X_{0}+Y_{0}\right), A_{\max }\right)$ corresponding to $\mathscr{D}_{0}$, and

$$
\begin{aligned}
& K(X, Y)=\left[\left(e^{-\tau}-A\right)\left(e^{-\tau}-B\right)\right]^{-1 / 2}|\Delta|^{-1 / 2} \\
& \cdot \frac{A(1-A) \sqrt{Y_{0}}\left[(1-A)^{2}-X_{0}\right]}{\sqrt{2 \pi}\left[Y_{0}-A\left(X_{0}+Y_{0}\right)\right]\left[Y_{0}+\left(1-X_{0}-Y_{0}\right) A-A^{2}\right]},
\end{aligned}
$$

where $\Delta$ is the Jacobian associated with (68), so that

$$
\begin{aligned}
\Delta & =X_{\tau} Y_{A}-X_{A} Y_{\tau}=\left(e^{-\tau}-1\right) \\
& \cdot\left\{\left[1-\frac{X_{0}}{(1-A)^{2}}\right]\left[-A e^{\tau}+\left(1-\frac{X_{0}}{1-A}\right) e^{-\tau}\right]\right. \\
& \left.+\frac{Y_{0}}{1-B}\left[1+\frac{X_{0}}{(1-A)^{2}} e^{-\tau}\right]\right\}=\left(e^{-\tau}-1\right)\left\{A+X_{0}\right. \\
& +Y_{0}-\frac{X_{0}}{1-A}+A e^{\tau}\left[\frac{X_{0}}{(1-A)^{2}}-1\right] \\
& \left.+e^{-\tau}\left[1-\frac{2 X_{0}}{1-A}+\frac{X_{0}\left(X_{0}+Y_{0}\right)}{(1-A)^{2}}\right]\right\}
\end{aligned}
$$

We can view (68) as representing a family of curves in the $(X, Y)$ plane, with $A$ indexing the family and $\tau$ increasing along a curve. When $\tau=0$ the curves in (68) meet at the corner point $(X, Y)=\left(X_{0}, Y_{0}\right)$ and we also note that the Jacobian in (71) vanishes when $\tau=0$, indicating a singularity in the transformation in (68). When $A=A_{\max }$ the curve becomes the horizontal segment $Y=Y_{0}$. But then (69) shows that $\left(1-A_{\max }\right)\left(A_{\max }+X_{0}+Y_{0}\right)=X_{0}$ so that $B=\infty$ in (67). Thus near $Y=Y_{0}$ the expansion in (68) becomes invalid. When $A=Y_{0} /\left(X_{0}+Y_{0}\right)$ the curve in (68) becomes

$$
\begin{aligned}
& Y=Y_{0}\left(1-\frac{1-e^{-\tau}}{X_{0}+Y_{0}}\right) \\
& X=\left(X_{0}+Y_{0}-1+e^{\tau}\right)\left(e^{-\tau}-\frac{Y_{0}}{X_{0}+Y_{0}}\right)
\end{aligned}
$$

and eliminating $\tau$ we see that (72) is precisely the curve $Y=\tilde{Y}(X)$ in $(56)$ that separates $\mathscr{D}_{0}$ from $\mathscr{D}_{+}$. For $A \epsilon$ $\left(A_{\text {min }}, Y_{0} /\left(X_{0}+Y_{0}\right)\right)$ the curves in (68) fill a portion of $\mathscr{D}_{+}$, but then the leading term for $\pi(k, r)$ is given by (60), and (65) corresponds to only an exponentially small correction to (60). When $A=A_{\text {min }}=1-\sqrt{X_{0}}$ the curve in (68) is tangent to the line $X=X_{0}$ at the point $Y=Y_{0}$, which will have significance for the parameter region $\mathscr{R}_{4}$. We also note that for $A \in$ $\left(Y_{0} /\left(X_{0}+Y_{0}\right), A_{\max }\right)$ when $\tau=-\log A$ the curves in (68) hit the $y$-axis (then $X=0$ by (68)) and then the first factor in (70) becomes singular, which indicates that the asymptotics become invalid. Along $A=Y_{0} /\left(X_{0}+Y_{0}\right)$ corresponding to $Y=\tilde{Y}(X), K(X, Y)$ in (70) is again singular. Thus (70) is singular when $A=Y_{0} /\left(X_{0}+Y_{0}\right), A=A_{\max }(B=\infty)$, and $e^{-\tau}=A$, corresponding to the three curves $(Y=\tilde{Y}(X)$, $Y=Y_{0}$, and $X=0$ ) that bound the region $\mathscr{D}_{0}$. We will give the appropriate expansions near these bounding curves in Section 3.3.

For $0<s<Y_{0}$ the curves in (62) fill the entire region $\mathscr{D}_{+}$, with $s=0$ corresponding to the line segment $Y=0,0<X<$ $X_{0}$, and $s=Y_{0}$ corresponding to the curve $Y=\tilde{Y}(X)$ (then (62) coincides with (72)). When $X=X_{0}$ we have $t=0$ and the curves in (62) hit the line $X=X_{0}$ at finite and nonzero slopes, for all $0 \leqslant s \leqslant Y_{0}$. As $t$ increases each curve will hit first either the $x$-axis or the $y$-axis. When $s=0$ the $x$-axis is hit first. For $1<X<X_{0}$ this occurs at a finite value of $t$, when $t=\log \left[\left(X_{0}-1\right) /(X-1)\right]$, but if $0<X<1$ in order to approach the $x$-axis we must let $s \rightarrow 0$ and $t \rightarrow \infty$ in such a way that $s e^{t}$ is held fixed. In this limit (62) may be approximated by $X \sim 1-s e^{t} / X_{0}$ and $Y \sim\left(X_{0}-1\right) s / X_{0}$. We discuss in more detail the behavior of (60) as $Y \rightarrow 0$ later, when we give the asymptotic expansion(s) for $\pi(k, r)$ that apply for $X_{0}>1$ and $r=O(1)$. When $s>0$ the curves in (62) hit the $y$-axis when $t=\log \left(1+X_{0} / s\right)$, for then $X=0$. In particular if $s=Y_{0}$ the corresponding curve hits the $y$-axis at $\tilde{Y}(0)$ in (57). Near both the $X$ - and $Y$-axes, (60) will have singular behaviors and other expansions must be constructed. Note, however, that (60) is not singular along the curve $Y=\tilde{Y}(X)$, whereas (65) 


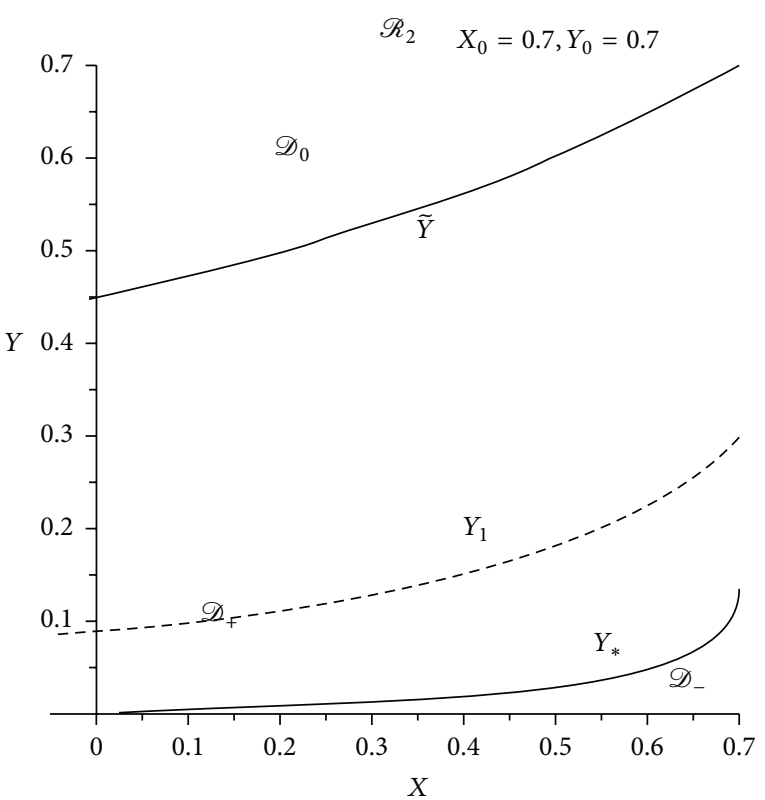

FIGURE 4: Region $\mathscr{R}_{2}$.

is singular. We can simplify (60) near $X=X_{0}$, and then we obtain the more explicit form

$$
\begin{aligned}
& \pi(k, r) \\
& \quad \sim \rho^{-1 / 2}\left(\frac{X_{0}}{X_{0}+Y}\right)^{m-k} \frac{\left(X_{0}+Y\right)^{2}-X_{0}}{\left(X_{0}+Y\right)^{5 / 2} \sqrt{2 \pi}} e^{\rho \Psi_{+}\left(X_{0}, Y\right)}, \\
& \Psi_{+}\left(X_{0}, Y\right)=Y+X_{0}-1-\left(X_{0}+Y\right) \log \left(X_{0}+Y\right),
\end{aligned}
$$

which holds for $m-k=\rho\left(X_{0}-X\right)=O(1)$ and $0<Y<Y_{0}$. However, we note that since $X_{0}>1$ we have $\Psi_{+}\left(X_{0}, Y\right)<$ 0 and thus $\pi(k, r)$ in (73) is exponentially small in $\rho$. This is true for the entire domains $\mathscr{D}_{0}$ and $\mathscr{D}_{+}$, as there is very little probability mass in these ranges if $X_{0}>1$.

We next consider regions $\mathscr{R}_{2}$ and $\mathscr{R}_{3}$ in parameter space, where it will become necessary to break up the state space into the three regions $\mathscr{D}_{+}, \mathscr{D}_{-}$, and $\mathscr{D}_{0}$. These regions are sketched in Figures 4 and 5. The curve that separates $\mathscr{D}_{+}$from $\mathscr{D}_{0}$ is again given by (56), while the curve separating $\mathscr{D}_{+}$from $\mathscr{D}_{-}$ will be

$$
\begin{aligned}
Y & =Y_{*}(X)=Y_{*}\left(X ; X_{0}\right)=\frac{1}{2}\left[2 \sqrt{X_{0}}-X_{0}-X\right. \\
& \left.-\sqrt{X_{0}-X} \sqrt{\left(2-\sqrt{X_{0}}\right)^{2}-X}\right]
\end{aligned}
$$

and thus, since now $X_{0}<1$,

$$
\begin{aligned}
Y_{*}(0) & =0, \\
Y_{*}\left(X_{0}\right) & =\sqrt{X_{0}}-X_{0} \\
& \left(\in\left(0, Y_{0}\right)\right) .
\end{aligned}
$$

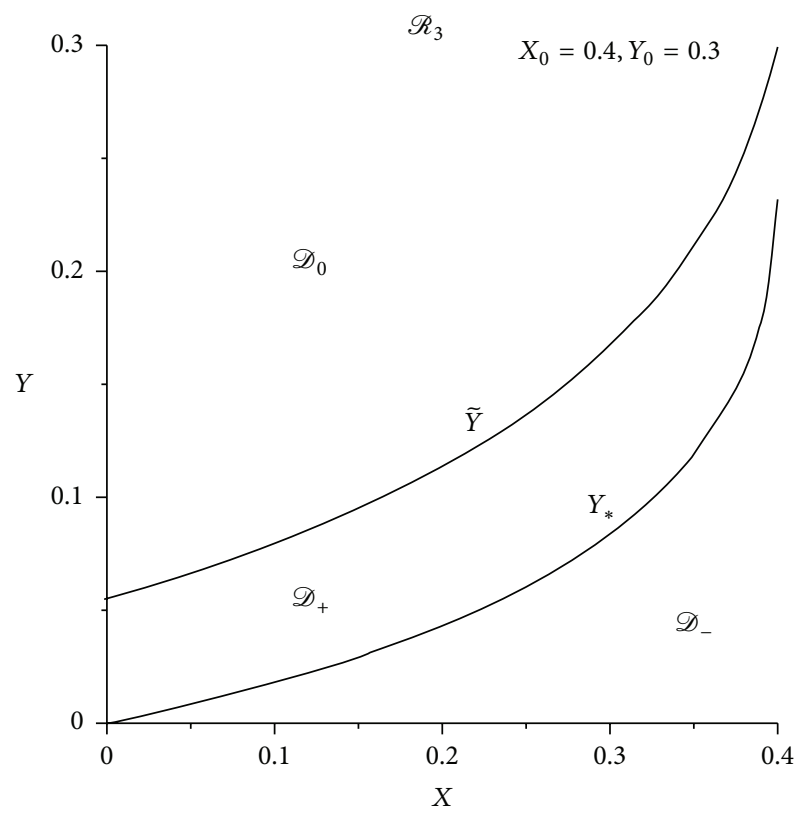

FIGURE 5: Region $\mathscr{R}_{3}$.

We thus define $\mathscr{D}_{-}$as

$$
\mathscr{D}_{-}=\left\{(X, Y): 0<X<X_{0}, 0<Y<Y_{*}(X)\right\}
$$

and, for regions $\mathscr{R}_{2}$ and $\mathscr{R}_{3}, \mathscr{D}_{+}$now becomes

$$
\mathscr{D}_{+}=\left\{(X, Y): 0<X<X_{0}, Y_{*}(X)<Y<\tilde{Y}(X)\right\} \text {, }
$$

with $\mathscr{D}_{0}$ still defined by (59).

Proposition 6. For $\left(X_{0}, Y_{0}\right) \in \mathscr{R}_{2} \cup \mathscr{R}_{3}$ the asymptotic expansions of $\pi(k, r)$ are as follows:

(i) $(X, Y) \in \mathscr{D}_{-}\left(0<Y<Y_{*}(X)\right)$

$$
\pi(k, r) \sim C_{1}(\rho) L(X, Y) e^{\rho \Phi(X, Y)} e^{\rho^{1 / 3} \Phi_{1}(X, Y)},
$$

where

$$
\begin{aligned}
& \Phi(X, Y)=W_{0} \log \left(W_{0}+s_{1}\right)+Y \log \left(Y+W_{0}\right)-Y \\
& \cdot \log Y-X \log \left[1-\sqrt{W_{0}} \frac{W_{0}+s_{1}}{W_{0}+Y}\right] \\
& -\frac{\sqrt{W_{0}}\left(W_{0}+s_{1}\right)}{W_{0}+Y}-\frac{1}{2} W_{0} \log W_{0}, \\
& s_{1}=s_{1}(X, Y)=W_{0}+\frac{Y+W_{0}}{2 \sqrt{W_{0}}}\left[W_{0}+1-X\right. \\
& \left.+\sqrt{\left(W_{0}+1-X\right)^{2}-4 W_{0}}\right],
\end{aligned}
$$




$$
\begin{aligned}
& \Phi_{1}(X, Y)=\Phi_{*}\left(s_{1}\right)=-r_{0} \frac{\left(1-\sqrt{X_{0}}\right)^{2 / 3}}{X_{0}^{1 / 6}} \\
& \cdot \log \left(\frac{s_{1}+W_{0}}{1-\sqrt{X_{0}}}\right) \\
& L(X, Y)=\frac{\left(W_{0}+s_{1}\right)^{3 / 2}}{\sqrt{Y} \sqrt{W_{0}+Y}} \frac{1}{\sqrt{s_{1}-Y}} \\
& \cdot \frac{\left(s_{1}+W_{0}\right) \sqrt{X_{0}}-s_{1}}{\sqrt{2 W_{0}+s_{1}+Y}}\left[1-\sqrt{W_{0}} \frac{W_{0}+s_{1}}{W_{0}+Y}\right]^{-1 / 2} \frac{1}{2 \pi} \\
& \cdot X_{0}^{-1 / 6}\left(1-\sqrt{X_{0}}\right)^{-5 / 6}\left[A i^{\prime}\left(r_{0}\right)\right]^{-2} \\
& \cdot \exp \left[-\frac{1+\sqrt{X_{0}}}{2 \sqrt{X_{0}}} \log \left(\frac{W_{0}+s_{1}}{1-\sqrt{X_{0}}}\right)\right] \text {, }
\end{aligned}
$$

with $W_{0}=\left(1-\sqrt{X_{0}}\right)^{2}$ and $r_{0}$ is the maximal root of the Airy function Ai(.). In (79), $C_{1} \sim \rho^{-2 / 3}$ for $\left(X_{0}, Y_{0}\right) \in$ $\mathscr{R}_{2}, C_{1}$ is given by (35) for $\left(X_{0}, Y_{0}\right) \in \mathscr{R}_{2} \cap \mathscr{R}_{3}$ (then $X_{0}+Y_{0}=1+\gamma / \sqrt{\rho}$ ), and $C_{1}$ is given by (36) for $\left(X_{0}, Y_{0}\right) \in \mathscr{R}_{3}$.

(ii) $(X, Y) \in \mathscr{D}_{+}\left(Y_{*}(X)<\mathrm{Y}<\tilde{Y}(X)\right)$.

The expression in (60) applies with $C \sim \rho^{-1 / 2}$ for $\left(X_{0}, Y_{0}\right) \in \mathscr{R}_{2}, C$ is given by (32) for $\left(X_{0}, Y_{0}\right) \in$ $\mathscr{R}_{2} \cap \mathscr{R}_{3}$, and $C$ is given by (33) for $\left(X_{0}, Y_{0}\right) \in \mathscr{R}_{3}$.

(iii) $(X, Y) \in \mathscr{D}_{0}\left(\tilde{Y}(X)<Y<Y_{0}\right)$.

The expression in (65) applies with $C_{0}$ given by (45) for $\left(X_{0}, Y_{0}\right) \in \mathscr{R}_{2}, C_{0}$ is given by (44) for $\left(X_{0}, Y_{0}\right) \in \mathscr{R}_{2} \cap$ $\mathscr{R}_{3}$, and $C_{0} \sim \rho^{-1 / 2}\left(1-X_{0}-Y_{0}\right)$ for $\left(X_{0}, Y_{0}\right) \in \mathscr{R}_{3}$.

In contrast to regions $\mathscr{D}_{+}$and $\mathscr{D}_{0}$, the expansion (79) in $\mathscr{D}_{-}$is a completely explicit function of $X, Y$, and $X_{0}$. We also note that $s_{1}(X, Y)$ has a simple linear dependence upon $Y$, and $s_{1}\left(X_{0}, Y\right)=Y$. In Section 4 we give a more geometric interpretation of this expansion, and we also observe that the form of (79) is slightly different from the expansions in $\mathscr{D}_{+}$and $\mathscr{D}_{0}$, as the former contains an additional factor that is of order $\exp \left[O\left(\rho^{1 / 3}\right)\right]$, and thus gives an additional subexponential dependence on $\rho$. While the forms of $C$, $C_{0}$, and $C_{1}$ change according to whether $\left(X_{0}, Y_{0}\right)$ lies in the regions $\mathscr{R}_{2}, \mathscr{R}_{3}$ or $\mathscr{R}_{2} \cap \mathscr{R}_{3}$ of parameter space, the ratios $C_{1}: C: C_{0}$ remain the same for these three cases.

The expansion in (79) is valid only in the interior of $\mathscr{D}_{-}$. As $Y \rightarrow 0$ there is a singularity due to the factor $1 / \sqrt{Y}$ in $L(X, Y)$ in (83). For $X \rightarrow X_{0}$ there is also a singularity due to the factor $1 / \sqrt{s_{1}-Y}$ in (83), and as $X \rightarrow X_{0}$, we find that $\left(s_{1}-Y\right)^{-1 / 2}=O\left[\left(X_{0}-X\right)^{-1 / 4}\right]$. The curve $Y=Y_{*}(X)$ that separates $\mathscr{D}_{+}$from $\mathscr{D}_{-}$corresponds to $s_{1}(X, Y)=\sqrt{X_{0}}-X_{0}$, and along $Y=Y_{*}(X)$ the factor $\left(s_{1}+W_{0}\right) \sqrt{X_{0}}-s_{1}=$ $-\left(1-\sqrt{X_{0}}\right)\left[s_{1}+X_{0}-\sqrt{X_{0}}\right]$ vanishes. Thus (83) shows that $L\left(X, Y_{*}(X)\right)=0$ which also indicates a nonuniformity in the asymptotics. Later we will give appropriate expansions near the three bounding curves $\left(Y=0, X=X_{0}\right.$, and $\left.Y=Y_{*}(X)\right)$ of region $\mathscr{D}_{-}$.

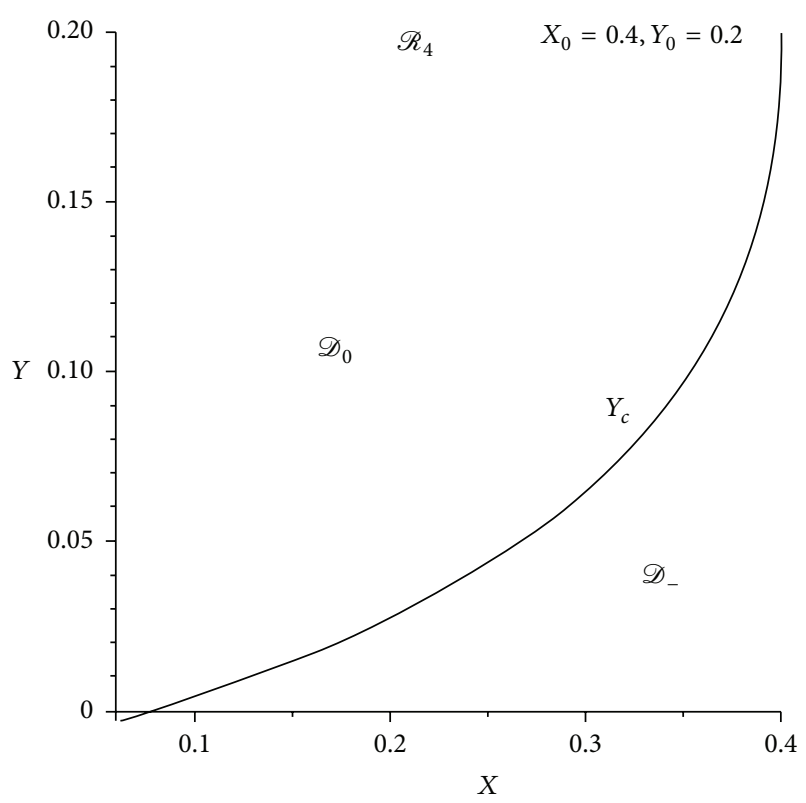

Figure 6: Region $\mathscr{R}_{4}$.

In Figure 4 we also indicate the curve

$$
Y=Y_{1}(X)=Y_{1}\left(X ; X_{0}\right)=\frac{\left(1-X_{0}\right)^{2}}{1-X}
$$

which lies entirely within $\mathscr{D}_{+}\left(\right.$when $\left.\left(X_{0}, Y_{0}\right) \in \mathscr{R}_{2}\right)$ and corresponds to $\partial \Psi_{+} / \partial Y=0$, where $\Psi_{+}$is in (61). We can also show that $\Psi_{+, Y Y}\left(X, Y_{1}(X)\right)<0$ so that, for a fixed $X \in\left(0, X_{0}\right)$, $\Psi_{+}$achieves a local maximum along $Y=Y_{1}(X)$. Note that $Y=Y_{1}(X)$ corresponds to $s(X, Y)=1-X_{0}\left(>\sqrt{X_{0}}-X_{0}\right)$ in (62), for then $X=1+\left(X_{0}-1\right) e^{t}$ and $Y=\left(1-X_{0}\right) e^{-t}$.

For $\left(X_{0}, Y_{0}\right) \in \mathscr{R}_{3}$ the curve in (84) plays no role, as it lies outside of $\mathscr{D}_{+}$, but now the curve

$$
Y=Y_{2}(X)=Y_{2}\left(X ; X_{0}, Y_{0}\right)=Y_{0} \frac{1-X_{0}}{1-X}
$$

lies within $\mathscr{D}_{0}$, connecting the points $\left(0, Y_{0}\left(1-X_{0}\right)\right)$ and $\left(X_{0}, Y_{0}\right)$. Along $Y=Y_{2}(X)$ we have $\Psi_{Y}=0$ and then $A=$ $1-X_{0}$ and $B=0$, so that (68) becomes $X=1-\left(1-X_{0}\right) e^{\tau}$ and $Y=Y_{0} e^{-\tau}$. Then for region $\mathscr{R}_{3}, \Psi$ will have a local maximum along $Y=Y_{2}$ in $\mathscr{D}_{0}$.

For $\left(X_{0}, Y_{0}\right) \in \mathscr{R}_{3}$ most of the mass will lie near the corner point $(X, Y)=\left(X_{0}, Y_{0}\right)$, where $\mathscr{D}_{0}$ and $\mathscr{D}_{+}$meet, but neither (60) nor (65) (with the appropriate $C_{0}$ and $C$ ) are valid there. For $\left(X_{0}, Y_{0}\right) \in \mathscr{R}_{2}, \pi(k, r)$ will be maximal near the point $(X, Y)=\left(X_{0}, 1-X_{0}\right)$, and (73) applies for $k=m-O(1)$ (or $X=X_{0}-O\left(\rho^{-1}\right)$ ) for both regions $\mathscr{R}_{1}$ and $\mathscr{R}_{2}$. By expanding (73) about $Y=1-X_{0}$, which is where $\Psi_{+}\left(X_{0}, Y\right)$ is maximal, we obtain precisely the expression in (49).

Next we consider region $\mathscr{R}_{4}$ of parameter space. Now the state space will be split into $\mathscr{D}_{0}$ and $\mathscr{D}_{-}$, and $\mathscr{D}_{+}$will be absent. This is sketched in Figure 6, and we also observe that as $X_{0}+Y_{0} \downarrow \sqrt{X_{0}}$ in region $\mathscr{R}_{3}$, the curves $Y_{*}(X)$ and $\tilde{Y}(X)$ in (75) and (56) become identical, and thus $\mathscr{D}_{+}$shrinks to 
this curve. For $\mathscr{R}_{4}$ there is a new curve that comes into play; namely,

$$
\begin{aligned}
Y & =Y_{c}(X)=Y_{c}\left(X ; X_{0}, Y_{0}\right)=-W_{0}+\frac{Y_{0}+W_{0}}{2 \sqrt{W_{0}}}\left[W_{0}\right. \\
& \left.+1-X-\sqrt{\left(W_{0}+1-X\right)^{2}-4 W_{0}}\right] .
\end{aligned}
$$

We have $Y_{c}\left(X_{0}\right)=Y_{0}$ and the curve hits the $x$-axis $(Y=0)$ when

$$
\begin{aligned}
Y_{c}(X) & =0 \Longleftrightarrow \\
X & =X_{0}-\frac{Y_{0}^{2}}{Y_{0}+W_{0}} \frac{1}{\sqrt{W_{0}}} .
\end{aligned}
$$

The curve $Y=Y_{c}(X)$ now separates $\mathscr{D}_{0}$ from $\mathscr{D}_{-}$and corresponds to $A=A_{\min }=1-\sqrt{X_{0}}$ in (68), and also $s_{1}(X, Y)=Y_{0}$ in $(81)$.

Proposition 7. For $\left(X_{0}, Y_{0}\right) \in \mathscr{R}_{4}$ (thus $0<Y_{0}<\sqrt{X_{0}}-X_{0}$ ) the asymptotic expansions of $\pi(k, r)$ are as follows:

(i) $(X, Y) \in \mathscr{D}_{-}, 0<Y<Y_{c}(X), X_{0}-Y_{0}^{2} /\left[\sqrt{W_{0}}\left(Y_{0}+\right.\right.$ $\left.\left.W_{0}\right)\right]<X<X_{0}$.

The expansion in (79) applies with now $C_{1}(\rho)$ given by (41).

(ii) $(X, Y) \in \mathscr{D}_{0}\left(\max \left\{0, Y_{c}(X)\right\}<Y<Y_{0}\right)$.

The expansion in (65) applies for $A \in\left(A_{\min }, A_{\max }\right)$ with $C_{0}(\rho) \sim \rho^{-1 / 2}\left(1-X_{0}-Y_{0}\right)$.

Again, different expansions must be given near $Y=Y_{c}(X)$ and near all boundaries of the state space. The curve $Y=$ $Y_{2}(X)$ in (85) still lies within $\mathscr{D}_{0}$ and is sketched in Figure 6, with again $\Psi_{Y}=0$ along this curve.

We conclude by noting that, for all regions of parameter space $\mathscr{R}_{j}$, the expansion in $\mathscr{D}_{0}$ depends upon the secondary storage capacity $Y_{0}$ (or $R$ ). For regions $\mathscr{R}_{1}$ and $\mathscr{R}_{2}$ the expansions in $\mathscr{D}_{+}$and $\mathscr{D}_{-}\left(\mathscr{R}_{2}\right.$ only) are independent of $Y_{0}$, except through the curve $\tilde{Y}(X)$ that bounds $\mathscr{D}_{+}$. Thus if $X_{0}+Y_{0}>1$ the effects of the finite storage capacity appear in $\mathscr{D}_{0}$ only, and then letting $Y_{0} \rightarrow \infty$ will recover the results for the storage model in $[10,11]$, which assumes that $R=\infty$. For $\left(X_{0}, Y_{0}\right) \in \mathscr{R}_{3}$, hence $\sqrt{X_{0}}<X_{0}+Y_{0}<1$, the expansions in $\mathscr{D}_{-}$and $\mathscr{D}_{+}$depend upon $Y_{0}$ only through the multiplicative constants $C_{1}$ and $C$, which now depend on $Y_{0}$ in view of (33) and (36). For $\left(X_{0}, Y_{0}\right) \in \mathscr{R}_{4}$, hence $0<Y_{0}<\sqrt{X_{0}}-X_{0}$, again the $\mathscr{D}_{-}$result depends on $Y_{0}$ only through the constant $C_{1}$ (cf. (41)). For $\left(X_{0}, Y_{0}\right) \in \mathscr{R}_{3} \cap \mathscr{R}_{4}$, that is, along and near the curve $X_{0}+Y_{0}=\sqrt{X_{0}}$, we have $Y_{3}(X)=Y_{c}(X)=\tilde{Y}(X)=Y_{*}(X)$ so all four of these curves coalesce. A special analysis is required for $X_{0}+Y_{0}-\sqrt{X_{0}}=O\left(\rho^{-1 / 3}\right)$ for values of $(X, Y)$ near this curve(s).

This completes our summary of the asymptotic expansions in the three main regions, $\mathscr{D}_{0}, \mathscr{D}_{+}$, and $\mathscr{D}_{-}$, of the state space.
3.3. Joint Distribution: Boundary, Corner, and Transition Regions. We next analyze the four boundary segments of the state space rectangle, namely, the line segments $\{Y=0,0<$ $\left.X<X_{0}\right\},\left\{X=X_{0}, 0<Y<Y_{0}\right\},\left\{X=0,0<Y<Y_{0}\right\}$, and $\left\{Y=Y_{0}, 0<X<X_{0}\right\}$. As we previously discussed, the expansions in the three main regions become invalid near the boundary of the state space, with the exception of the $\mathscr{D}_{+}$expansion in (60), which remains valid near $X=X_{0}$, reducing to (73) when $k=m-O(1)\left(X=X_{0}-O\left(\rho^{-1}\right)\right)$, at least for $\left(X_{0}, Y_{0}\right) \in \mathscr{R}_{1} \cup \mathscr{R}_{2}$. For the four boundary segments we will typically consider the respective scales $r=O(1)$, $k=m-O(1), k=O(1)$, and $r=R-O(1)$, though at times a different scaling must be considered in addition to these discrete scales. Note also that a particular point on a boundary segment may also require a separate expansion, and this occurs in $\mathscr{R}_{2} \cup \mathscr{R}_{3}$, when $Y_{*}(X)$ hits $X=X_{0}$ at the point $\left(X_{0}, \sqrt{X_{0}}-X_{0}\right)$. Also, in $\mathscr{R}_{1} \cup \mathscr{R}_{2} \cup \mathscr{R}_{3}$, the curve $\tilde{Y}(X)$ that separates $\mathscr{D}_{0}$ from $\mathscr{D}_{+}$hits the $y$-axis when $Y=Y_{0}\left[1-X_{0} /\left(X_{0}+Y_{0}\right)^{2}\right](>0)$ and this point requires a separate analysis. Finally, in $\mathscr{R}_{4}$ the curve $Y_{c}(X)$ hits the $x$ axis when $X=X_{0}-Y_{0}^{2} /\left[\left(Y_{0}+W_{0}\right) \sqrt{W_{0}}\right]$.

After treating the four boundary segments we will give asymptotic results that are valid near the four corner points, $(0,0),\left(X_{0}, 0\right),\left(X_{0}, Y_{0}\right)$, and $\left(0, Y_{0}\right)$, of the state space. Finally we give results for points $(X, Y)$ that lie on or near the transition curves $\tilde{Y}(X)$ (for $\mathscr{R}_{1} \cup \mathscr{R}_{2} \cup \mathscr{R}_{3}$ ), $Y_{*}(X)$ (for $\mathscr{R}_{2} \cup$ $\mathscr{R}_{3}$ ), and $Y_{c}(X)$ (for $\left.\mathscr{R}_{4}\right)$. Unlike the previous subsections where we listed the results by region $\mathscr{R}_{j}$ of parameter space, here we go by region of state space, and each proposition will correspond to one such region and the different results for the different $\mathscr{R}_{j}$ will be collected in that proposition.

Proposition 8. For $r=O(1)$ and $k=\rho X, 0<X<X_{0}$, one has the following expansions:

$$
\begin{aligned}
& \text { (i) } \mathscr{R}_{1}: X_{0}>1,0<X<1 \\
& \pi(k, r) \sim \rho^{-r} \frac{X_{0}-1}{2 \pi \sqrt{X X_{0}}}(1-X)^{-r}\left(X_{0}-1\right)^{-2 r} \\
& \cdot(r-1) ! e^{\rho\left(-2+X+X_{0}-X \log X-X_{0} \log X_{0}\right)}, \quad r \geqslant 1, \\
& \pi(k, 0)-\rho^{k} \frac{e^{-\rho}}{k !} \sim \rho^{-1} \frac{-X e^{\rho\left(-2+X+X_{0}-X \log X-X_{0} \log X_{0}\right)}}{2 \pi \sqrt{X X_{0}}\left(X_{0}-1\right)(1-X)} . \\
& \text { (ii) } \mathscr{R}_{1}: X_{0}>1, X=1+\alpha / \sqrt{\rho}, \alpha=O(1) \\
& \pi(k, r) \sim \rho^{-r / 2} \frac{X_{0}-1}{2 \pi \sqrt{X_{0}}}\left(X_{0}-1\right)^{-2 r} \\
& \cdot\left[\int_{-\alpha}^{\infty}(\alpha+u)^{r-1} e^{-u^{2} / 2} d u\right] e^{\rho\left(-1+X_{0}-X_{0} \log X_{0}\right)}, \\
& \pi(k, 0)-\rho^{k} \frac{e^{-\rho}}{k !} \sim \rho^{-1 / 2} \\
& \cdot \frac{-e^{\rho\left(-1+X_{0}-X_{0} \log X_{0}\right)}}{2 \pi \sqrt{X_{0}}\left(X_{0}-1\right)}\left[\int_{-\alpha}^{\infty} e^{-u^{2} / 2} d u\right] .
\end{aligned}
$$


(iii) $\mathscr{R}_{1}: X_{0}>1,1<X<X_{0}$

$$
\begin{aligned}
& \pi(k, r) \sim \rho^{-1 / 2} \frac{\left(X_{0}-1\right)^{3}}{\sqrt{2 \pi X_{0}}}(X-1)^{r-1} \\
& \cdot\left[X-1+\left(X_{0}-1\right)^{2}\right]^{-r-1} e^{\rho\left(-1+X_{0}-X_{0} \log X_{0}\right)},
\end{aligned}
$$

$r \geqslant 1$,

$$
\begin{aligned}
\pi(k, 0)-\rho^{k} \frac{e^{-\rho}}{k !} \sim \rho^{-1 / 2} \\
\cdot \frac{-\left(X_{0}-1\right) e^{\rho\left(-1+X_{0}-X_{0} \log X_{0}\right)}}{\sqrt{2 \pi X_{0}}\left[X-1+\left(X_{0}-1\right)^{2}\right]} .
\end{aligned}
$$

(iv) $\mathscr{R}_{1} \cap \mathscr{R}_{2}: X_{0}=1+\beta / \sqrt{\rho}, \beta=O(1), 0<X<1$

$$
\begin{aligned}
\pi(k, r) \sim & \rho^{-1 / 2} \frac{1}{\sqrt{X}} e^{\rho(-1+X-X \log X)} \frac{1}{2 \pi i} \\
& \cdot \int_{B r_{+}} \frac{\Gamma(z+r)(1-X)^{z}}{r ! D_{z}(-\beta) D_{z-1}(-\beta)} d z
\end{aligned}
$$

where $\mathrm{Br}_{+}$is the imaginary axis in the $z$-plane if $r \geqslant 1$, and if $r=0$ the contour is to be indented to the right of the pole at $z=0$. Here $\Gamma(\cdot)$ is the gamma function. When $\beta=0$ the expression in (94) simplifies to

$\pi(k, r)$

$$
\sim \rho^{-1 / 2} \sqrt{\frac{2}{\pi}} \frac{(1-X)}{\sqrt{X}}(2-X)^{-r-1} e^{\rho(-1+X-X \log X)} .
$$

(v) $\mathscr{R}_{2} \cup \mathscr{R}_{3}: 0<X_{0}<1, X_{0}+Y_{0}>\sqrt{X_{0}}, 0<X<X_{0}$

$$
\begin{aligned}
& \pi(k, r) \sim \sqrt{\rho} C_{1}(\rho) e^{\rho \Phi(X, 0)} e^{\rho^{1 / 3} \Phi_{*}\left(s_{1}^{0}\right)} \frac{\rho^{r} W_{0}^{r}}{r !} \\
& \cdot \frac{\left(1-\sqrt{X_{0}}\right)^{1 / 6}\left(W_{0}+s_{1}^{0}\right) \sqrt{\sqrt{X_{0}}-X_{0}-s_{1}^{0}}}{\sqrt{2 \pi}\left[\operatorname{Ai}^{\prime}\left(r_{0}\right)\right]^{2} X_{0}^{1 / 6} \sqrt{s_{1}^{0}\left(s_{1}^{0}+2 W_{0}\right)}}\left(\frac{\sqrt{W_{0}}}{s_{1}^{0}+W_{0}}\right)^{1 / 2 \sqrt{X_{0}}},
\end{aligned}
$$

where $W_{0}=\left(1-\sqrt{X_{0}}\right)^{2}$,

$$
\begin{aligned}
& s_{1}^{0} \\
& =-W_{0} \\
& \quad+\frac{\sqrt{W_{0}}}{2}\left[W_{0}+1-X+\sqrt{\left(W_{0}+1-X\right)^{2}-4 W_{0}}\right],
\end{aligned}
$$

so that $s_{1}^{0}$ corresponds to $s_{1}(X, 0)$ in $(81), \Phi_{*}(\cdot)$ is defined in (82), and $\Phi(\mathrm{X}, 0)$ can be computed from (80) by setting $Y=0$ and replacing $s_{1}$ by $s_{1}^{0}$. The value of $C_{1}$ in (96) is given by (34) for $\mathscr{R}_{2}$, by (35) for $\mathscr{R}_{2} \cap \mathscr{R}_{3}\left(X_{0}+Y_{0}=1+O\left(\rho^{-1 / 2}\right)\right)$, and by (36) for $\mathscr{R}_{3}$.

(vi) $\mathscr{R}_{4}: 0<Y_{0}<\sqrt{X_{0}}-X_{0}, X_{0}-\left(Y_{0}^{2} /\left[\sqrt{W_{0}}\left(Y_{0}+W_{0}\right)\right]\right)<$ $X<X_{0}$.

The expression in (96) applies with $C_{1}$ now given by (41). For region $\mathscr{R}_{3} \cap \mathscr{R}_{4}$ (hence $X_{0}+Y_{0}=\sqrt{X_{0}}+$ $\left.O\left(\rho^{-1 / 3}\right)\right)$, (96) holds for $0<X<X_{0}$, where $C_{1}$ can be computed from (37).

(vii) $\mathscr{R}_{4}: 0<Y_{0}<\sqrt{X_{0}}-X_{0}, 0<X<X_{0}-Y_{0}^{2} /\left[\sqrt{W_{0}}\left(Y_{0}+\right.\right.$ $\left.\left.W_{0}\right)\right] \equiv X_{L}$

$\pi(k, r) \sim\left(1-X_{0}-Y_{0}\right) \frac{\rho^{r}}{r !}\left(\frac{Y_{0} B_{0}}{1-B_{0}}\right)^{r} g(X) e^{\rho \Psi(X, 0)}$,

$\Psi(X, 0)$

$$
\begin{aligned}
= & A_{0}\left(1-\frac{1}{B_{0}}\right)+X_{0} \log \left(1-A_{0}\right) \\
& +Y_{0} \log \left(1-B_{0}\right)-X \log \left(1-\frac{A_{0}}{B_{0}}\right),
\end{aligned}
$$

where $A_{0}=A_{0}(X)=A_{0}\left(X ; X_{0}, Y_{0}\right)$ is the solution of the algebraic equation

$$
\begin{aligned}
& \left(A_{0}-1+X_{0}+Y_{0}\right) \\
& \cdot\left[Y_{0}\left(1-A_{0}\right)^{2}+X_{0} A_{0}\left(1-X_{0}-A_{0}\right)\right]=(-X) \\
& \cdot\left(1-X_{0}-A_{0}\right)\left[\left(1-A_{0}\right)\left(A_{0}+X_{0}+Y_{0}\right)-X_{0}\right]
\end{aligned}
$$

that satisfies $A_{0}(0)=1-X_{0}-Y_{0}$ and $A_{0}\left(X_{L}\right)=1-$ $\sqrt{X_{0}}=\sqrt{W_{0}}$,

$$
B_{0}=\frac{A_{0}\left(1-X_{0}-A_{0}\right)}{\left(1-A_{0}\right)\left(A_{0}+X_{0}+Y_{0}\right)-X_{0}},
$$

so that $B_{0}(0)=1-X_{0}-Y_{0}$ and $B_{0}\left(X_{L}\right)=W_{0} /\left(W_{0}+Y_{0}\right)$, and

$$
\begin{aligned}
& g(X)=\frac{A_{0}\left(1-A_{0}\right)\left[\left(1-A_{0}\right)^{2}-X_{0}\right] Y_{0}}{\left(1-B_{0}\right) \sqrt{B_{0}-A_{0}}\left[Y_{0}-A_{0}\left(X_{0}+Y_{0}\right)\right]\left[Y_{0}-A_{0}^{2}+\left(1-X_{0}-Y_{0}\right) A_{0}\right]}\left\{A_{0}+X_{0}+Y_{0}-\frac{X_{0}}{1-A_{0}}\right. \\
& \left.\quad+\frac{A_{0}}{B_{0}}\left[\frac{X_{0}}{\left(1-A_{0}\right)^{2}}-1\right]+B_{0}\left[1-\frac{2 X_{0}}{1-A_{0}}+\frac{X_{0}\left(X_{0}+Y_{0}\right)}{\left(1-A_{0}\right)^{2}}\right]\right\}^{-1 / 2} .
\end{aligned}
$$


For $X \rightarrow 0$ and $X \rightarrow X_{L}$ one can obtain $g(X)$ more explicitly, with

$$
\begin{array}{r}
g(X) \sim \sqrt{\frac{X_{0}+Y_{0}}{X}}, \quad X \longrightarrow 0, \\
g(X) \sim \frac{2}{Y_{0}} \frac{\left(W_{0}+Y_{0}\right)^{3 / 2}\left(1-\sqrt{X_{0}}\right)^{5 / 2}\left(X_{L}-X\right)}{\left(2 W_{0}+Y_{0}\right)^{3 / 2}\left(\sqrt{X_{0}}-X_{0}-Y_{0}\right)^{3 / 2}}, \\
X \rightarrow X_{L} .
\end{array}
$$

(viii) $\mathscr{R}_{4}: 0<Y_{0}<\sqrt{X_{0}}-X_{0}, X-X_{L}=\rho^{-1 / 3} \Lambda=O\left(\rho^{-1 / 3}\right)$

$$
\begin{gathered}
\pi(k, r) \sim\left(1-X_{0}-Y_{0}\right) \frac{\rho^{r} W_{0}^{r}}{r !} \rho^{-1 / 3} \\
\cdot \frac{\sqrt{W_{0}+Y_{0}} X_{0}^{1 / 6}\left(1-\sqrt{X_{0}}\right)^{5 / 6}}{\sqrt{2 W_{0}+Y_{0}}\left(\sqrt{X_{0}}-X_{0}-Y_{0}\right)^{3 / 2}} \exp \left[\rho \Psi\left(X_{L}, 0\right)\right. \\
\left.+\rho^{2 / 3} \Psi_{X}\left(X_{L}, 0\right) \Lambda+\frac{1}{2} \rho^{1 / 3} \Psi_{X X}\left(X_{L}, 0\right) \Lambda^{2}\right] \exp \left[\frac{\Lambda^{3}}{6}\right. \\
\left.\cdot \frac{W_{0}\left(Y_{0}+W_{0}\right)^{3}\left(Y_{0}^{3}+3 W_{0} Y_{0}^{2}+4 W_{0}^{2} Y_{0}-2 \sqrt{X_{0}} W_{0}^{5 / 2}\right)}{Y_{0}^{3}\left(Y_{0}+2 W_{0}\right)^{3}\left(\sqrt{X_{0}}-X_{0}-Y_{0}\right)^{2}}\right] \\
\cdot \frac{1}{2 \pi i} \int_{-i \infty}^{i \infty} \frac{1}{A i(\theta)} \exp \left[\frac{\left(Y_{0}+W_{0}\right)\left(1-\sqrt{X_{0}}\right)^{5 / 3}}{Y_{0}\left(Y_{0}+2 W_{0}\right) X_{0}^{1 / 6}} \Lambda \theta\right] d \theta,
\end{gathered}
$$

where

$$
\begin{gathered}
\Psi\left(X_{L}, 0\right)=Y_{0} \log \left(\frac{Y_{0}}{W_{0}+Y_{0}}\right)-\frac{Y_{0}}{\sqrt{W_{0}}}+\frac{1}{2} X_{0} \\
\cdot \log X_{0}+\left(\frac{Y_{0}^{2}}{Y_{0}+W_{0}} \frac{1}{\sqrt{W_{0}}}-X_{0}\right) \\
\cdot \log \left(\frac{\sqrt{X_{0}}-X_{0}-Y_{0}}{\sqrt{W_{0}}}\right), \\
\Psi_{X}\left(X_{L}, 0\right)=-\log \left(\frac{\sqrt{X_{0}}-X_{0}-Y_{0}}{1-\sqrt{X_{0}}}\right) \\
\Psi_{X X}\left(X_{L}, 0\right)=-\frac{\left(Y_{0}+W_{0}\right)^{2} \sqrt{W_{0}}}{Y_{0}\left(Y_{0}+2 W_{0}\right)\left(\sqrt{X_{0}}-X_{0}-Y_{0}\right)} .
\end{gathered}
$$

We note that the regions of validity of the expansions in Proposition 8 are such that the four corner points of the state space are excluded. For regions $\mathscr{R}_{1}$ and $\mathscr{R}_{2}$ the expansions for $r=O(1)$ are independent of the secondary storage capacity $Y_{0}$. For region $\mathscr{R}_{3}$ the result in (96) does depend upon $Y_{0}$, but only through the multiplicative constant $C_{1}$, which now depends on $Y_{0}$ in view of (36). This is also true for region $\mathscr{R}_{4}$ (and $\mathscr{R}_{3} \cap \mathscr{R}_{4}$ ) when $X>X_{L}$, as then $C_{1}$ is given by
(41) or (37). However, for $0<X<X_{L}$ and $X \approx X_{L}$, the expressions in (98) and (105) show that now $\pi(k, r)$ depends in an intricate way on $Y_{0}$. Thus the finiteness of the secondary storage capacity affects the probabilities that there are even a few secondary spaces occupied.

For region $\mathscr{R}_{1}, \pi(k, r)$ is exponentially small for $r \geqslant 1$ and $\pi(k, 0)$ approximately follows a Poisson distribution, as indicated previously in Proposition 3. The results in (88)(93) better quantify (47) and estimate the exponentially small error from the Poisson approximation. For regions $\mathscr{R}_{2}, \mathscr{R}_{3}$, and $\mathscr{R}_{4}, \pi(k, r)$ is always exponentially small for $r=O(1)$, and the approximations do not distinguish $r=0$ from $r \geqslant 1$. By evaluating the contour integral in (105) for $\Lambda \rightarrow+\infty$ we can verify that (105) asymptotically matches to (96) (with $C_{1}$ given by (41)), as $X \downarrow X_{L}$. Similarly, for $\Lambda \rightarrow-\infty$ (105) will match to (98) as $X \uparrow X_{L}$.

Next we consider points $(k, r)$ near $X=X_{0}$, with $X_{0}-X=$ $o(1)$. Note that only regions $\mathscr{D}_{+}$and $\mathscr{D}_{-}$can be bounded by $X=X_{0}$, for $0<Y<Y_{0}$.

Proposition 9. For $X \sim X_{0}$, one uses the scales $k=m-n$ and $X=X_{0}-\rho^{-2 / 3} v_{1}$, so that $n=\rho^{1 / 3} v_{1}=\rho\left(X_{0}-X\right)$ denotes the number of empty primary storage spaces. The expansions are now the following:

(i) $\mathscr{R}_{2} \cup \mathscr{R}_{3}: 0<X_{0}<1, X_{0}+Y_{0}>\sqrt{X_{0}}$; $n=O(1)$, $0<Y<\sqrt{X_{0}}-X_{0}$

$$
\pi(k, r) \sim \rho^{-1 / 6} C_{1}(\rho) e^{\rho \Phi\left(X_{0}, Y\right) e^{\rho^{1 / 3}} \Phi_{*}(Y)}\left(\sqrt{X_{0}}\right)^{n}
$$

$$
\begin{aligned}
& \cdot \frac{1}{\sqrt{2 \pi}} \frac{1}{A i^{\prime}\left(r_{0}\right)}\left(1-\sqrt{X_{0}}\right)^{-1 / 6} \\
& \cdot X_{0}^{-5 / 6}\left[n+\frac{\sqrt{X_{0}}\left(Y+W_{0}\right)}{\left(Y+W_{0}\right) \sqrt{X_{0}}-Y}\right] \\
& \cdot \frac{\left(Y+W_{0}\right) \sqrt{X_{0}}-Y}{\sqrt{Y}} \\
& \cdot \exp \left[-\frac{1+\sqrt{X_{0}}}{2 \sqrt{X_{0}}} \log \left(\frac{W_{0}+Y}{1-\sqrt{X_{0}}}\right)\right],
\end{aligned}
$$

where $C_{1}$ is given by (34), (35), and (36) for parameter regions $\mathscr{R}_{2}, \mathscr{R}_{2} \cap \mathscr{R}_{3}$, and $\mathscr{R}_{3}$, respectively, $\Phi_{*}(Y)$ is obtained by replacing $s_{1}$ by $s_{1}\left(X_{0}, Y\right)=Y$ in (82), and

$$
\begin{aligned}
\Phi\left(X_{0}, Y\right)= & \left(Y+W_{0}\right) \log \left(Y+W_{0}\right)-Y \log Y \\
& +\sqrt{X_{0}}-1-\frac{1}{2} X_{0} \log X_{0} \\
& -\frac{1}{2} W_{0} \log W_{0} .
\end{aligned}
$$


(ii) $\mathscr{R}_{2} \cup \mathscr{R}_{3}: v_{1}=O(1)$ with $v_{1}>0,0<Y<\sqrt{X_{0}}-X_{0}$ $\pi(k, r) \sim \rho^{-1 / 6} C_{1}(\rho) e^{\rho \Phi\left(X_{0}, Y\right)} e^{\rho^{1 / 3}\left[\nu_{1} \log \left(\sqrt{X_{0}}\right)+\Phi_{*}(Y)\right]}$

$$
\begin{aligned}
& \cdot \frac{1}{\sqrt{2 \pi}} \frac{1}{\left[A i^{\prime}\left(r_{0}\right)\right]^{2}} \frac{\left(Y+W_{0}\right) \sqrt{X_{0}}-Y}{\left[Y X_{0}\left(1-\sqrt{X_{0}}\right)\right]^{1 / 2}} \\
& \cdot \exp \left[-\frac{1+\sqrt{X_{0}}}{2 \sqrt{X_{0}}} \log \left(\frac{W_{0}+Y}{1-\sqrt{X_{0}}}\right)\right] \\
& \cdot A i\left(\nu+r_{0}\right),
\end{aligned}
$$

where $v=\left(1-\sqrt{X_{0}}\right)^{1 / 3} X_{0}^{-1 / 3} v_{1}$ and $C_{1}$ is again given in Proposition 2 for the different ranges $\mathscr{R}_{j}$.

(iii) $\mathscr{R}_{3} \cap \mathscr{R}_{4}$ or $\mathscr{R}_{4}: n=O(1)$ or $\nu_{1}=O(1)$.

Now the expressions in (109) and (111) apply over all $Y\left(0<Y<Y_{0}\right)$, with $C_{1}$ given by (37) for $\mathscr{R}_{3} \cap \mathscr{R}_{4}$ and (41) for $\mathscr{R}_{4}$.

(iv) $\mathscr{R}_{2} \cup \mathscr{R}_{3}: v_{1}=O(1), Y-\left(\sqrt{X_{0}}-X_{0}\right)=\rho^{-1 / 3} \omega_{1}=$ $O\left(\rho^{-1 / 3}\right)$

$$
\begin{aligned}
& \pi(k, r) \sim \rho^{-1 / 6} C_{1}(\rho) e^{\rho\left[-1+\sqrt{X_{0}}-\sqrt{X_{0}} \log \left(\sqrt{X_{0}}\right)\right]} \\
& \cdot \exp \left\{-\rho^{2 / 3} \omega_{1} \log \left(\sqrt{X_{0}}\right)\right. \\
& \left.+\rho^{1 / 3}\left[\nu_{1} \log \left(\sqrt{X_{0}}\right)-\frac{\omega_{1}^{2}}{2 \sqrt{X_{0}}}\right]\right\} \frac{\left(1-\sqrt{X_{0}}\right)^{1 / 3}}{\sqrt{2 \pi} X_{0}^{7 / 12}} \\
& \cdot \exp \left[\frac{1+\sqrt{X_{0}}}{6 X_{0}\left(1-\sqrt{X_{0}}\right)} \omega_{1}^{3}\right] \frac{1}{2 \pi i} \\
& \cdot \int_{-i \infty}^{i \infty} \frac{A i(\nu+\theta)}{A i^{2}(\theta)} e^{-\omega \theta} d \theta,
\end{aligned}
$$

where $\omega=\left(1-\sqrt{X_{0}}\right)^{-1 / 3} X_{0}^{-1 / 6} \omega_{1}$, so that $\nu_{1} \omega_{1}=$ $\sqrt{X_{0}} v \omega$, and $C_{1}$ is given again in Proposition 2 for the three cases $\mathscr{R}_{2}, \mathscr{R}_{2} \cap \mathscr{R}_{3}$, and $\mathscr{R}_{3}$.

(v) $\mathscr{R}_{1} \cup \mathscr{R}_{2} \cup \mathscr{R}_{3}: 0<Y<Y_{0}\left(\mathscr{R}_{1}\right)$ or $\sqrt{X_{0}}-X_{0}<Y<$ $Y_{0}\left(\mathscr{R}_{2} \cup \mathscr{R}_{3}\right), n=O(1)$

$\pi(k, r)$

$$
\sim C(\rho) \frac{\left(X_{0}+Y\right)^{2}-X_{0}}{\sqrt{2 \pi}\left(X_{0}+Y\right)^{5 / 2}}\left(\frac{X_{0}}{X_{0}+Y}\right)^{n} e^{\rho \Psi_{+}\left(X_{0}, Y\right)},
$$

where $\Psi_{+}\left(X_{0}, Y\right)$ is given in (74) and $C$ has the expansions in (31)-(33).

Note that the asymptotics are most complicated in (112), which occurs when $(X, Y) \approx\left(X_{0}, \sqrt{X_{0}}-X_{0}\right)$ and this is where the curve $Y_{*}(X)$ hits the line $X=X_{0}$. This intersection occurs only for regions $\mathscr{R}_{2}$ and $\mathscr{R}_{3}$. The integrand in (112) is a meromorphic function of $\theta$, having double poles at all roots of the Airy function $\mathrm{Ai}(\cdot)$.
Proposition 10. For $k=O(1)$ and $r=\rho Y, 0<Y<Y_{0}$, one has the following expansions:

(i) $\mathscr{R}_{1} \cup \mathscr{R}_{2} \cup \mathscr{R}_{3}: 0<Y<Y_{0}\left[1-X_{0} /\left(X_{0}+Y_{0}\right)^{2}\right] \equiv Y_{U}$ $\pi(k, r)$

$$
\sim \sqrt{\rho} C(\rho) e^{\rho \Psi_{+}(0, Y)} \frac{\rho^{k}}{k !} \frac{\left(1+Y-D_{0}^{2}\right)^{k}\left(1-D_{0}\right) Y}{D_{0} \sqrt{X_{0}\left[Y+2 D_{0}^{2}\left(1-D_{0}\right)\right]}},
$$

where $D_{0}=D_{0}(Y)$ is the root of the cubic equation

$$
D_{0}^{3}-2 D_{0}^{2}+\left(1-X_{0}-Y\right) D_{0}+Y=0
$$

that satisfies $D_{0}(0)=1-\sqrt{X_{0}}=\sqrt{W_{0}}$ and

$$
\begin{aligned}
\Psi_{+}(0, Y)= & D_{0}+\frac{X_{0}}{1-D_{0}}-2-X_{0} \log X_{0}-Y \log Y \\
& +2 X_{0} \log \left(1-D_{0}\right)+2 Y \log D_{0} .
\end{aligned}
$$

The expansion of $C$ is given by (31)-(33) according to subregions of $\mathscr{R}_{1} \cup \mathscr{R}_{2} \cup \mathscr{R}_{3}$.

(ii) $\mathscr{R}_{1} \cup \mathscr{R}_{2} \cup \mathscr{R}_{3} \cup \mathscr{R}_{4}: Y_{U}<Y<Y_{0}\left(\mathscr{R}_{1} \cup \mathscr{R}_{2} \cup \mathscr{R}_{3}\right)$ or $0<Y<Y_{0}\left(\mathscr{R}_{4}\right)$

$$
\begin{gathered}
\pi(k, r) \sim Q(0,0) e^{\rho \Psi(0, Y)} \frac{\rho^{k}}{k !}\left[\frac{A_{1}\left(A_{1}+X_{0}-2\right)+1}{1-A_{1}}\right]^{k} \\
\cdot \frac{\sqrt{Y_{0} A_{1}} \sqrt{A_{1}\left(A_{1}+X_{0}-2\right)+1}\left[\left(1-A_{1}\right)^{2}-X_{0}\right]}{\sqrt{Y\left(Y_{0}-Y\right)} \sqrt{\left|\Delta_{L}(Y)\right|}\left[Y_{0}-A_{1}\left(X_{0}+Y_{0}\right)\right]},
\end{gathered}
$$

where

$$
\begin{aligned}
& A_{1}=A_{1}(Y)=A_{1}\left(Y ; X_{0}, Y_{0}\right)=\frac{1}{2}\left[1-X_{0}-Y_{0}\right. \\
& \left.\quad+\sqrt{\left(X_{0}+Y_{0}-1\right)^{2}+4 Y}\right], \\
& \Psi(0, Y)=A_{1}-1+X_{0} \log \left(1-A_{1}\right)+\left(Y_{0}-Y\right) \\
& \quad \cdot \log \left(1-A_{1}\right)+Y \log A_{1}-Y \log Y-\left(Y_{0}-Y\right) \\
& \quad \cdot \log \left(Y_{0}-Y\right)+Y_{0} \log Y_{0}, \\
& -\Delta_{L}(Y)=\left(1-A_{1}\right)\left\{A_{1}+X_{0}+Y_{0}+\frac{X_{0}}{\left(1-A_{1}\right)^{2}}\right. \\
& \quad-\frac{X_{0}}{1-A_{1}}-1 \\
& \left.\quad+A_{1}\left[1-\frac{2 X_{0}}{1-A_{1}}+\frac{X_{0}\left(X_{0}+Y_{0}\right)}{\left(1-A_{1}\right)^{2}}\right]\right\}
\end{aligned}
$$

and the expansion of $Q(0,0)$ is given in Proposition 1 for the three cases $\mathscr{R}_{1} \cup \mathscr{R}_{2}, \mathscr{R}_{2} \cap \mathscr{R}_{3}$, and $\mathscr{R}_{3} \cup \mathscr{R}_{4}$ (when $\left.Q(0,0) \sim 1-X_{0}-Y_{0}\right)$. 
(iii) $\mathscr{R}_{1} \cup \mathscr{R}_{2} \cup \mathscr{R}_{3}: Y-Y_{U}=y_{*} / \sqrt{\rho}=O\left(\rho^{-1 / 2}\right)$

$$
\begin{aligned}
& \pi(k, r) \sim Q(0,0) e^{\rho \Psi(0, \tilde{Y}(0))} e^{\sqrt{\rho} y_{*} \Psi_{Y}(0, \tilde{Y}(0))} \sqrt{\rho} \\
& \cdot \frac{\rho^{k}}{k !}\left[Y_{0}+\frac{X_{0}}{X_{0}+Y_{0}}\right]^{k} \exp \left[\frac{1}{2} y_{*}^{2} \Psi_{+, Y Y}(0, \tilde{Y}(0))\right] \\
& \cdot \sqrt{\frac{X_{0}}{Y_{0}}} \\
& \cdot \frac{\left(X_{0}+Y_{0}\right)^{2}-X_{0}}{\sqrt{\left(X_{0}+Y_{0}\right)^{3}+X_{0}\left(Y_{0}-X_{0}\right)}}\left(\int_{a_{c} y_{*}}^{\infty} e^{-u^{2} / 2} d u\right),
\end{aligned}
$$

where

$$
\begin{aligned}
& \Psi(0, \tilde{Y}(0)) \\
& =X_{0} \log X_{0}+Y_{0} \log Y_{0}-\left(X_{0}+Y_{0}\right) \log \left(X_{0}+Y_{0}\right)-\frac{X_{0}}{X_{0}+Y_{0}} \\
& \quad+\frac{X_{0} Y_{0}}{\left(X_{0}+Y_{0}\right)^{2}}\left[2 \log \left(X_{0}+Y_{0}\right)-\log Y_{0}\right] \\
& \quad+Y_{0}\left[\frac{X_{0}}{\left(X_{0}+Y_{0}\right)^{2}}-1\right] \log \left[1-\frac{X_{0}}{\left(X_{0}+Y_{0}\right)^{2}}\right], \\
& \Psi_{Y}(0, \tilde{Y}(0))=\Psi_{+, Y}(0, \tilde{Y}(0)) \\
& =\log Y_{0}-\log \left[\left(X_{0}+Y_{0}\right)^{2}-X_{0}\right], \\
& \Psi_{+, Y Y}(0, \tilde{Y}(0)) \\
& =\frac{X_{0}}{Y_{0}} \frac{\left(X_{0}+Y_{0}\right)^{2}}{\left(X_{0}+Y_{0}\right)^{3}+X_{0}\left(Y_{0}-X_{0}\right)}\left[1-\frac{Y_{0}}{X_{0}} \frac{\left(X_{0}+Y_{0}\right)^{2}+X_{0}}{\left(X_{0}+Y_{0}\right)^{2}-X_{0}}\right],
\end{aligned}
$$

$a_{c}$

$$
=\frac{\left(X_{0}+Y_{0}\right)^{5 / 2} \sqrt{\left(X_{0}+Y_{0}\right)^{2}-X_{0}}}{\sqrt{X_{0} Y_{0}} \sqrt{\left(X_{0}+Y_{0}\right)^{3}+X_{0}\left(Y_{0}-X_{0}\right)} \sqrt{\left(X_{0}+Y_{0}\right)^{2}+Y_{0}-X_{0}}} .
$$

$$
\pi(k, r) \sim Q(0,0) e^{\rho \Psi\left(X, Y_{0}\right)} \frac{\rho^{p}}{p !}\left[Y_{0}\left(e^{\tau_{U}}-1\right)\right]^{p} \frac{\sqrt{1-A_{\max }}}{\sqrt{e^{-\tau_{U}-A_{\max }}}}\left[\frac{\left(1-X_{0}-Y_{0}\right)^{2}+4 Y_{0}}{\left(1-Y_{0}-X\right)^{2}-4 A_{\max }\left(1-X_{0} /\left(1-A_{\max }\right)\right)}\right]^{1 / 4}
$$

where $\tau_{U}=\tau_{U}(X)=\tau_{U}\left(X ; X_{0}, Y_{0}\right)$ is given by

$$
\begin{aligned}
\tau_{U} & =-\log \left[1-Y_{0}-X\right. \\
& \left.-\sqrt{\left(1-Y_{0}-X\right)^{2}-4 A_{\max }\left(1-\frac{X_{0}}{1-A_{\max }}\right)}\right] \\
& +\log \left[2\left(1-\frac{X_{0}}{1-A_{\max }}\right)\right],
\end{aligned}
$$

where $A_{\max }$ is given in (69), $Q(0,0)$ has the respective expansions in (28)-(30), and

$$
\Psi\left(X, Y_{0}\right)=A_{\max }\left(1-e^{\tau_{U}}\right)+X_{0} \log \left(1-A_{\max }\right)
$$

We see that for each of the three cases the dependence of $\pi(k, r)$ on $k$ is of the form $\rho^{k}\left[A_{*}(Y)\right]^{k} / k$ ! (times a function of $Y$ or $\left.y_{*}\right)$, where the geometric ratio $A_{*}$ depends upon $Y=r / \rho$ and undergoes a transition when $Y$ increases past $Y_{U}=\tilde{Y}(0)$, which can occur only for regions $\mathscr{R}_{1}, \mathscr{R}_{2}$, and $\mathscr{R}_{3}$. We note that $A_{1}\left(Y_{U}\right)=Y_{0} /\left(X_{0}+Y_{0}\right)$ and $D_{0}\left(Y_{U}\right)=Y_{0} /\left(X_{0}+Y_{0}\right)$, so that $A_{*}$ is continuous along $Y=Y_{U}$, with $A_{*}\left(Y_{U}\right)=1+$ $Y_{0}-X_{0} /\left(X_{0}+Y_{0}\right)$, as indicated in (121). The expansion in (117) develops a singularity as $Y \downarrow Y_{U}$, in view of the factor $\left[Y_{0}-A_{1}\left(X_{0}+Y_{0}\right)\right]^{-1}$. The expansion is also singular as $Y \uparrow Y_{0}$ (for all regions $\mathscr{R}_{j}$ ) and as $Y \downarrow 0$ (for region $\mathscr{R}_{4}$ only). Then we are approaching the corner points $\left(0, Y_{0}\right)$ or $(0,0)$ of the state space. Using the expansions

$$
\begin{aligned}
& \int_{a_{c} y_{*}}^{\infty} e^{-u^{2} / 2} d u \\
& \quad \sim \begin{cases}\sqrt{2 \pi}, & y_{*} \longrightarrow-\infty \\
\left(a_{c} y_{*}\right)^{-1} \exp \left(-\frac{a_{c}^{2} y_{*}^{2}}{2}\right), & y_{*} \longrightarrow+\infty\end{cases}
\end{aligned}
$$

we can easily show that (121) matches to (117) (for region $\mathscr{R}_{1} \cup$ $\mathscr{R}_{2} \cup \mathscr{R}_{3}$ in the intermediate limit where $y_{*} \rightarrow+\infty$ and $Y \downarrow$ $Y_{U}$ ) and to (114) (with now $y_{*} \rightarrow-\infty$ and $Y \uparrow Y_{U}$ ). Note also that the ratio $Q(0,0) / C(\rho)$ is asymptotically the same for each of the three regions $\mathscr{R}_{1}, \mathscr{R}_{2}$, and $\mathscr{R}_{3}$, in view of (28)(33). For any region $\mathscr{R}_{j}$, for $k=O(1)$ and $Y \in\left(0, Y_{0}\right), \pi(k, r)$ is exponentially small in $\rho$.

Proposition 11. For $R-r=p=O(1)$ and $0<X<X_{0}$ one has (for all regions $\mathscr{R}_{j}$ in parameter space)

$$
\begin{aligned}
& {\left[\left(1-Y_{0}-X\right)^{2}-4 A_{\max }\left(1-X_{0} /\left(1-A_{\max }\right)\right)\right]} \\
& -Y_{0} \tau_{U}-X \log \left(1-A_{\max } e^{\tau_{U}}\right) .
\end{aligned}
$$

Thus (127) gives the expansion when there are only a few secondary spaces empty and a fraction $X / X_{0}=k / m \in(0,1)$ of primary spaces occupied. The expressions can be simplified in the limits $X \rightarrow 0$ (then $\left.\tau_{U}(0)=-\log \left(A_{\max }\right)\right)$ and $X \rightarrow X_{0}$ (then $\tau_{U}\left(X_{0}\right)=0$ ), but then other "corner" expansions will apply. Note that (127) is a completely explicit expression in terms of $p$ and $X$.

Next we examine the four corners of the state space, where $(X, Y)=\left(0, Y_{0}\right),(0,0),\left(X_{0}, 0\right)$, and $\left(X_{0}, Y_{0}\right)$. We recall that these ranges are important in that for region $\mathscr{R}_{1} \cap \mathscr{R}_{2}\left(X_{0} \approx\right.$ 1) most of the mass concentrates near the corner $\left(X_{0}, 0\right)$, while for region $\mathscr{R}_{3} \cup \mathscr{R}_{4}\left(X_{0}+Y_{0}<1\right)$ most of the mass 
occurs near $\left(X_{0}, Y_{0}\right)$. We will start with the corner $\left(0, Y_{0}\right)$ and proceed about the perimeter of the state space in a counterclockwise manner.

Proposition 12. For $k=O(1), p=R-r=O(1)$, and any parameter region $\mathscr{R}_{j}$ one has

$$
\begin{gathered}
\pi(k, r) \sim \sqrt{2 \pi \rho} Q(0,0) \frac{\rho^{k+p}}{k ! p !}\left(1+Y_{0}\right)^{k} \\
\cdot\left[\frac{Y_{0}\left(1-A_{\max }\right)}{A_{\max }}\right]^{p} \\
\cdot e^{\rho \Psi\left(0, Y_{0}\right)} \sqrt{\frac{1-A_{\max }}{A_{\max }}}\left[\left(1-X_{0}-Y_{0}\right)^{2}+4 Y_{0}\right]^{1 / 4},
\end{gathered}
$$

where $Q(0,0)$ is given in Proposition $1, A_{\max }$ is in (69), and

$$
\begin{aligned}
\Psi\left(0, Y_{0}\right)= & A_{\max }-1+X_{0} \log \left(1-A_{\max }\right) \\
& +Y_{0} \log \left(A_{\max }\right) .
\end{aligned}
$$

This gives the expansion when there are but a few primary spaces occupied and a few secondary spaces empty. Next we consider the corner point $(0,0)$, and this will typically correspond to $k, r=O(1)$. The results will be very different for region $\mathscr{R}_{1}$ compared to those for the remaining regions.

Proposition 13. For $k, r=O(1)$ and $\rho \rightarrow \infty$ one has

(i) $\left(X_{0}, Y_{0}\right) \in \mathscr{R}_{1}\left(X_{0}>1\right)$

$\pi(k, r)$

$$
\sim \sqrt{\frac{\rho}{2 \pi}} \frac{(r-1) !}{k !} \rho^{k-r} \frac{\left(X_{0}-1\right)^{1-2 r}}{\sqrt{X_{0}}} e^{\rho\left(-2+X_{0}-X_{0} \log X_{0}\right)},
$$

$$
r \geqslant 1
$$

$\pi(k, 0)-\frac{\rho^{k} e^{-\rho}}{k !} \sim-\sqrt{\frac{\rho}{2 \pi}} \frac{\rho^{k}}{(k-1) !} \frac{e^{\rho\left(-2+X_{0}-X_{0} \log X_{0}\right)}}{X_{0}-1}$.

(ii) $\left(X_{0}, Y_{0}\right) \in \mathscr{R}_{1} \cap \mathscr{R}_{2}\left(X_{0}-1=\beta / \sqrt{\rho}=O\left(\rho^{-1 / 2}\right)\right)$, $r \geqslant 0$

$\pi(k, r) \sim \frac{\rho^{k} e^{-\rho}}{k ! r !} \frac{1}{\sqrt{2 \pi} i} \int_{\mathrm{Br}_{+}} \frac{\Gamma(z+r)}{D_{z}(-\beta) D_{z-1}(-\beta)} d z$,

where $B r_{+}$is as in (94), and when $\beta=0$

$$
\pi(k, r) \sim \frac{\rho^{k} e^{-\rho}}{k !} 2^{-r} \quad(\beta=0) .
$$

(iii) $\left(X_{0}, Y_{0}\right) \in \mathscr{R}_{2} \cup \mathscr{R}_{3}\left(X_{0}<1, Y_{0}+X_{0}>\sqrt{X_{0}}\right)$

$$
\pi(k, r) \sim \rho^{2 / 3} C_{1}(\rho) \frac{\rho^{k+r} e^{-\rho}}{k ! r !} W_{0}^{r}\left(1-W_{0}\right)^{k},
$$

where $C_{1}$ is given by (34), (35), or (36) for $\mathscr{R}_{2}, \mathscr{R}_{2} \cap \mathscr{R}_{3}$, or $\mathscr{R}_{3}$, respectively.

$$
\begin{aligned}
\text { (iv) }\left(X_{0}, Y_{0}\right) \in \mathscr{R}_{4}\left(0<Y_{0}<\sqrt{X_{0}}-X_{0}\right) \\
\pi(k, r) \sim \sqrt{2 \pi \rho} \sqrt{X_{0}+Y_{0}}\left(1-X_{0}-Y_{0}\right) \\
\cdot e^{\rho\left[-X_{0}-Y_{0}+\left(X_{0}+Y_{0}\right) \log \left(X_{0}+Y_{0}\right)\right]} \\
\cdot \frac{\rho^{k+r}}{k ! r !}\left[Y_{0}+\frac{X_{0}}{X_{0}+Y_{0}}\right]^{k}\left[\frac{Y_{0}}{X_{0}+Y_{0}}-Y_{0}\right]^{r} .
\end{aligned}
$$

(v) $\left(X_{0}, Y_{0}\right) \in \mathscr{R}_{2} \cup \mathscr{R}_{3} ; k=\rho^{2 / 3} X^{\prime}, r=\rho^{2 / 3} Y^{\prime} ; X=$ $\rho^{-1 / 3} X^{\prime}, Y=\rho^{-1 / 3} Y^{\prime}$$$
\pi(k, r) \sim \rho^{2 / 3} C_{1}(\rho) \frac{\rho^{k+r}}{k ! r !} W_{0}^{r}\left(1-W_{0}\right)^{k} \frac{1}{2 \pi i}
$$$$
\int_{-i \infty}^{i \infty} \frac{e^{-\xi^{\prime} z}}{[A i(z)]^{2}} d z
$$$$
\cdot \exp \left\{\frac{1}{2} \rho^{1 / 3}\left[\frac{\left(Y^{\prime}\right)^{2}}{W_{0}}-\frac{W_{0}}{\left(1-W_{0}\right)^{2}}\left(X^{\prime}\right)^{2}\right]\right.
$$$$
\left.-\frac{1}{6}\left[\frac{\left(Y^{\prime}\right)^{3}}{W_{0}^{2}}+\frac{W_{0}\left(W_{0}+2\right)}{\left(1-W_{0}\right)^{4}}\left(X^{\prime}\right)^{3}\right]\right\} \text {, }
$$$$
\xi^{\prime}=\frac{\left(1-\sqrt{X_{0}}\right)^{2 / 3}}{X_{0}^{1 / 6}}\left[\frac{Y^{\prime}}{W_{0}}-\frac{X^{\prime}}{1-W_{0}}\right] \text {. }
$$

We note that (136) and (137) are continuous along the curve $X_{0}+Y_{0}=\sqrt{X_{0}}$, corresponding to $\mathscr{R}_{3} \cap \mathscr{R}_{4}$, in view of the expansion in (36) for $C_{1}$ in $\mathscr{R}_{3}$. Thus the leading term for $k, r=O(1)$ for $\left(X_{0}, Y_{0}\right) \in \mathscr{R}_{3} \cap R_{4}$ can be obtained by either using (36) to compute $C_{1}$ in (136) or replacing $Y_{0}$ by $\sqrt{X_{0}}-X_{0}$ in (137). The scale $k, r=O\left(\rho^{2 / 3}\right)$ must be considered (cf. (138)), as the approximation in (136) cannot directly match to those for $k=O(1), 0<Y<Y_{U}$ (cf. (114)) or $r=O(1)$, $0<X<X_{0}$ (cf. (96)). We can view (136) as a special case of (138), letting $X^{\prime}, Y^{\prime} \rightarrow 0$ in the latter and noting that

$$
(2 \pi i)^{-1} \int_{-i \infty}^{i \infty}[\mathrm{Ai}(z)]^{-2} d z=1
$$

For region $\mathscr{R}_{4}$ we see from Figure 6 that only state space region $\mathscr{D}_{0}$ meets the corner $(X, Y)=(0,0)$. Then $(137)$ matches directly to (98), in the limits $k \rightarrow \infty, X \rightarrow 0$, and to (117), in the limits $r \rightarrow \infty, Y \rightarrow 0$. Note that, for region $\mathscr{R}_{4}$, $A_{1}(0)=1-X_{0}-Y_{0}$, which follows from (118) (this is true also for $\mathscr{R}_{3}$, but then (117) applies only for $Y>Y_{U}$ and thus not near the corner $(0,0))$. We can obtain a result analogous to (138) for the transitional range $\mathscr{R}_{3} \cap \mathscr{R}_{4}$, but we do not give that for the sake of brevity.

Proposition 14. For $k=m-O(1)$ and $r=O(1)$, or for $k=$ $m-O(\sqrt{\rho})$ and $r=O(\sqrt{\rho})$, the expansions of $\pi(k, r)$ are as follows: 
(i) $\left(X_{0}, Y_{0}\right) \in \mathscr{R}_{1}, m-k=n \geqslant 0$

$\pi(k, r) \sim \frac{1}{\sqrt{2 \pi \rho X_{0}}} e^{\rho\left(-1+X_{0}-X_{0} \log X_{0}\right)}\left(X_{0}-1\right) X_{0}^{-r-1}$,

$r \geqslant 1$

$\pi(k, 0) \sim \frac{1}{\sqrt{2 \pi \rho X_{0}}} e^{\left(-1+X_{0}-X_{0} \log X_{0}\right)}\left(X_{0}^{n}-\frac{1}{X_{0}}\right)$.

(ii) $\left(X_{0}, Y_{0}\right) \in \mathscr{R}_{1} \cap \mathscr{R}_{2}\left(X_{0}-1=\beta / \sqrt{\rho}\right), m-k=n \geqslant 0$

$\pi(k, r)$

$\sim \rho^{-1} \rho^{-z_{0} / 2} \frac{e^{-\beta^{2} / 4}}{r ! \Delta_{0}(\beta)} \Gamma\left(z_{0}+r+1\right)\left[\frac{n z_{0}}{z_{0}+r}+1\right]$,

where $z_{0}$ is the minimal root of the parabolic cylinder function $D_{z}(\cdot)$; that is, $z_{0}=z_{0}(\beta)=\min \{z$ : $\left.D_{z}(-\beta)=0\right\}$, and $\Delta_{0}(\beta)=-\left.(d / d z) D_{z}(-\beta)\right|_{z=z_{0}(\beta)}$.

(iii) $\left(X_{0}, Y_{0}\right) \in \mathscr{R}_{1} \cap \mathscr{R}_{2}\left(X_{0}-1=\beta / \sqrt{\rho}\right), k=\rho+\sqrt{\rho} \alpha$, and $r=\sqrt{\rho} \Omega$, with $\alpha \leqslant \beta$ and $\Omega>0$

$\pi(k, r) \sim \rho^{-1} \frac{1}{2 \pi i} \int_{-i \infty}^{i \infty} \frac{e^{-\alpha^{2} / 4} D_{z}(-\alpha) \Omega^{z-1}}{D_{z}(-\beta) D_{z-1}(-\beta)} d z$

which when $\beta=0$ simplifies to

$\pi(k, r) \sim \rho^{-1} \sqrt{\frac{2}{\pi}}(\Omega-\alpha) e^{-(\Omega-\alpha)^{2} / 2} ;$

$$
\alpha<0, \Omega>0 \text {. }
$$

(iv) $\left(X_{0}, Y_{0}\right) \in \mathscr{R}_{1} \cap \mathscr{R}_{2}\left(X_{0}-1=\beta / \sqrt{\rho}\right), k=\rho+\sqrt{\rho} \alpha$, $\alpha<\beta, r=O(1)$

$\pi(k, r)$

$$
\sim \rho^{-1 / 2} \rho^{-z_{0} / 2} e^{-\alpha^{2} / 4} \frac{\Gamma\left(r+z_{0}\right)}{r !} \frac{D_{z_{0}}(-\alpha)}{\Delta_{0}(\beta) D_{z_{0}-1}(-\beta)} .
$$

(v) $\left(X_{0}, Y_{0}\right) \in \mathscr{R}_{1} \cap \mathscr{R}_{2}$ with now $X_{0}-1=\beta_{*} \rho^{-1 / 4}=$ $O\left(\rho^{-1 / 4}\right)$ and $\beta_{*}>0, r=O(1), k=\rho+\sqrt{\rho} \alpha, \alpha=O(1)$

$\pi(k, r) \sim \frac{\beta_{*}^{3}}{2 \pi \rho^{1 / 4}} \otimes_{*} \int_{-\alpha}^{\infty} \frac{(u+\alpha)^{r-1}}{\left(u+\alpha+\beta_{*}^{2}\right)^{r+1}} e^{-u^{2} / 2} d u$

$r \geqslant 1$

$\pi(k, 0)-\frac{\rho^{k} e^{-\rho}}{k !} \sim-\frac{\beta_{*}}{2 \pi \rho^{1 / 4}} \otimes_{*} \int_{-\alpha}^{\infty} \frac{e^{-u^{2} / 2}}{u+\alpha+\beta_{*}^{2}} d u$,

$\otimes_{*}=\exp \left[-\frac{\sqrt{\rho}}{2} \beta_{*}^{2}+\frac{\rho^{1 / 4}}{6} \beta_{*}^{3}-\frac{1}{12} \beta_{*}^{4}\right]$. (vi) $\left(X_{0}, Y_{0}\right) \in \mathscr{R}_{2} \cup \mathscr{R}_{3} \cup \mathscr{R}_{4}, m-k=n \geqslant 0$

$$
\begin{gathered}
\pi(k, r) \sim \rho^{1 / 3} C_{1}(\rho) \frac{\rho^{r} W_{0}^{r}}{r !}\left(\sqrt{X_{0}}\right)^{n}(n+1) \\
\cdot e^{\rho \Phi\left(X_{0}, 0\right)} e^{\rho^{1 / 3} \Phi_{*}(0)} \frac{\left(1-\sqrt{X_{0}}\right)^{4 / 3}}{A i^{\prime}\left(r_{0}\right) X_{0}^{1 / 3}} \\
\cdot \exp \left[-\frac{1}{2 \sqrt{X_{0}}} \log \left(1-\sqrt{X_{0}}\right)\right],
\end{gathered}
$$

$\Phi_{*}(0)=-r_{0} \frac{\left(1-\sqrt{X_{0}}\right)^{2 / 3}}{X_{0}^{1 / 6}} \log \left(1-\sqrt{X_{0}}\right)$,

$\Phi\left(X_{0}, 0\right)=\frac{1}{2} W_{0} \log \left(X_{0}\right)-\frac{1}{2} X_{0} \log \left(X_{0}\right)+\sqrt{X_{0}}$

$-1$

and $C_{1}$ is given in Proposition 2 for the different regions $\mathscr{R}_{j}$.

For region $\mathscr{R}_{1}$, (141) show that the dependence of $\pi(k, r)$ on $m-k$ and $r$ is quite simple, but we have to distinguish the cases $r=0$ and $r \geqslant 1$. The same is true for regions $\mathscr{R}_{2}, \mathscr{R}_{3}$, and $\mathscr{R}_{4}$, where (149) applies for all $r \geqslant 0$, except now the form of $C_{1}$ in (149) is different for the five cases in Proposition 2. However, when $X_{0} \approx 1$, the asymptotic structure of $\pi(k, r)$ is quite complicated, and we must consider separately the scales $X_{0}-1=O\left(\rho^{-1 / 2}\right)(m-\rho=O(\sqrt{\rho}))$ and $X_{0}-1=$ $O\left(\rho^{-1 / 4}\right)\left(m-\rho=O\left(\rho^{3 / 4}\right)\right)$. For $X_{0}-1=O\left(\rho^{-1 / 2}\right)$ we obtain the limiting density in (53) or (143), as the limit of $\rho \pi(\rho+\sqrt{\rho} \alpha, \sqrt{\rho} \Omega)=\rho \pi(m+\sqrt{\rho}(\alpha-\beta), \sqrt{\rho} \Omega)$ for $\rho \rightarrow \infty$. This expansion applies for $\Omega>0$, for any $\alpha \leqslant \beta$, but becomes invalid as $\Omega \rightarrow 0$. For $\Omega \rightarrow 0$ the asymptotic behavior of the contour integral in (143) is determined by the singularity at $z=z_{0}(\beta)$, and the density behaves as $O\left(\Omega^{z_{0}-1}\right)$ for $\Omega \rightarrow 0$ and $\alpha<\beta$. This corresponds to either an integrable singularity or a zero of the density (unless $\beta=0$ then $z_{0}=1$ ) and in either case indicates a nonuniformity in the asymptotics. Thus we need the expansion in (145) for $r=O(1)$. For $r \rightarrow \infty$ we have, by Stirling's formula,

$$
\frac{\Gamma\left(r+z_{0}\right)}{r !}=\frac{\Gamma\left(r+z_{0}\right)}{\Gamma(r+1)} \sim r^{z_{0}-1}=(\sqrt{\rho} \Omega)^{z_{0}-1}
$$

and then (145) matches to (143) in the intermediate limit where $r \rightarrow \infty$ but $\Omega=r / \sqrt{\rho} \rightarrow 0$. The expansion in (145) itself breaks down when $\alpha \rightarrow \beta$, since by the definition of $z_{0}(\beta)$ we have $D_{z_{0}(\beta)}(-\alpha) \rightarrow 0$ as $\alpha \uparrow \beta$. Then for $\alpha \sim \beta$ we have the expansion in (142), which holds for $n=O(1)$ and we note that $n=m-k=(\beta-\alpha) / \sqrt{\rho}$ so that $\alpha=\beta-n \rho^{-1 / 2}$.

We can show also that the expansions for $n, r=O(1)$ (cf. (141), (142), and (149)) match in appropriate intermediate limits. Consider (142) for $\beta \rightarrow-\infty$ (then we are moving into the range $\left.X_{0}<1\right)$. We now have $z_{0} \rightarrow \infty$ and more precisely

$$
z_{0}(\beta)=\frac{\beta^{2}}{4}-r_{0}\left(-\frac{\beta}{2}\right)^{2 / 3}-\frac{1}{2}+o(1), \quad \beta \longrightarrow-\infty
$$


so the approximation to the minimal root of the parabolic cylinder function $D_{z}(-\beta)$ involves the maximal root of the Airy function $\operatorname{Ai}(\cdot)$. Thus, for $\beta \rightarrow-\infty$ we have

$$
\Delta_{0}(\beta) \sim z_{0}^{z_{0} / 2} e^{-z_{0} / 2}\left(\frac{2}{-\beta}\right)^{1 / 3} \sqrt{2 \pi} \mathrm{Ai}^{\prime}\left(r_{0}\right)
$$

which can be obtained by approximated $D_{z}(-\beta)$ by Airy functions in the double limit where $\beta \rightarrow-\infty$ and $z \rightarrow$ $\infty$, with $z-\beta^{2} / 4=O\left[(-\beta)^{2 / 3}\right]$. Using (154) in (142) and expanding $\Gamma\left(z_{0}(\beta)+r+1\right)$ by Stirling's formula (since now $\left.z_{0} \rightarrow \infty\right)$ for a fixed $r$, (142) becomes

$$
\rho^{-1} \rho^{-z_{0} / 2}(n+1)\left(-\frac{\beta}{2}\right)^{4 / 3} \frac{e^{-\beta^{2} / 4}}{\operatorname{Ai}^{\prime}\left(r_{0}\right)} z_{0}^{z_{0} / 2} e^{-z_{0} / 2} \frac{z_{0}^{r}}{r !}
$$

Then (155) must agree with the expansion of (149) as $X_{0} \uparrow 1$. Then we use the fact that $\rho^{1 / 3} C_{1} \sim \rho^{-1 / 3}$ in region $\mathscr{R}_{2}$. Also, as $X_{0} \rightarrow 1, W_{0}=\left(1-\sqrt{X_{0}}\right)^{2} \sim \beta^{2} /(4 \rho)$,

$$
\begin{aligned}
& \rho\left[-1+\sqrt{X_{0}}-\frac{1}{2} X_{0} \log X_{0}+W_{0} \log \left(1-\sqrt{X_{0}}\right)\right] \\
& =\frac{\beta^{2}}{4} \log \left(\frac{-\beta}{2 \sqrt{\rho}}\right)-\frac{3}{8} \beta^{2}+o(1), \\
& -\rho^{1 / 3} r_{0} X_{0}^{-1 / 6}\left(1-\sqrt{X_{0}}\right)^{2 / 3} \log \left(1-\sqrt{X_{0}}\right) \\
& \sim-r_{0}\left(-\frac{\beta}{2}\right)^{2 / 3} \log \left(-\frac{\beta}{2 \sqrt{\rho}}\right) .
\end{aligned}
$$

Using (156) we see that as $X_{0} \uparrow 1$, (149) becomes

$$
\begin{aligned}
& \rho^{-1 / 3} \frac{1}{r !}\left(\frac{\beta^{2}}{4}\right)^{r} \frac{(n+1)}{\mathrm{Ai}^{\prime}\left(r_{0}\right)}\left(-\frac{\beta}{2 \sqrt{\rho}}\right)^{4 / 3} \\
& \cdot \exp \left\{\left[\frac{\beta^{2}}{4}-r_{0}\left(-\frac{\beta}{2}\right)^{2 / 3}-\frac{1}{2}\right] \log \left(-\frac{\beta}{2 \sqrt{\rho}}\right)\right. \\
& \left.-\frac{3}{8} \beta^{2}\right\} .
\end{aligned}
$$

But by using (153) in (155) we obtain again the expression in (157), which verifies the matching.

Now consider (141) for $X_{0} \downarrow 1$ and (142) for $\beta \rightarrow+\infty$. We now have

$$
\begin{aligned}
& z_{0}(\beta) \sim \frac{\beta}{\sqrt{2 \pi}} e^{-\beta^{2} / 2}, \\
& \Delta_{0}(\beta) \sim \frac{\sqrt{2 \pi}}{\beta} e^{\beta^{2} / 4},
\end{aligned}
$$

$$
\beta \longrightarrow+\infty
$$

so that $z_{0}$ is exponentially small and $\Delta_{0}$ is exponentially large. The last factor in (142), namely, $n z_{0} /\left(z_{0}+r\right)+1$, can be approximated by 1 if $r \geqslant 1$ and is equal to $n+1$ if $r=0$. Now also $\Gamma\left(z_{0}+r+1\right) / r ! \rightarrow 1$ and (142) becomes, for $\beta \rightarrow+\infty$,

$$
\frac{\beta}{\sqrt{2 \pi}} e^{-\beta^{2} / 2} \rho^{-1} \begin{cases}1, & r \geqslant 1 \\ n+1, & r=0,\end{cases}
$$

and the above clearly agrees with (141), when we expand these for $X_{0} \rightarrow 1$.

The results in (146)-(148) assume the scaling $X_{0}-1=$ $\beta_{*} \rho^{-1 / 4}$, and these are needed to asymptotically connect the parameter ranges $X_{0}-1=O\left(\rho^{-1 / 2}\right)$ and $X_{0}>1$. It is only near the corner $(X, Y)=\left(X_{0}, 0\right)$ that we must consider this scaling (and indeed also the $\beta$-scale). For other ranges of $X$ and $r=$ $O(1)$, we can get the expansions of $\pi(k, r)$ as limiting cases of other expansions, as they lie in the asymptotic matching range where $X_{0} \rightarrow 1$ but $\left(X_{0}-1\right) \sqrt{\rho}(=\beta) \rightarrow \infty$. For a fixed $\beta_{*}>0$ and $0<X<1$ we have

$$
\pi(k, r) \sim \frac{(r-1) ! \beta_{*}^{1-2 r} \rho^{-r / 2} \otimes_{*}}{2 \pi \sqrt{X} \rho^{1 / 4}(1-X)^{r}} e^{\rho(-1+X-X \log X)},
$$

$$
r \geqslant 1
$$

for $X-1=\alpha_{*} \rho^{-1 / 4}=O\left(\rho^{-1 / 4}\right)$ with $0<\alpha_{*}<\beta_{*}$ we have

$$
\pi(k, r) \sim \frac{\otimes_{*}}{\sqrt{2 \pi} \rho^{3 / 4}} \frac{\beta_{*}^{3}}{\alpha_{*}^{2}}, \quad r \geqslant 1 ;
$$

and for the scale $m-k=n=O(1)$ we have $\alpha_{*} \sim \beta_{*}$ so the last factor in (161) may be approximated by $\beta_{*}^{3} \alpha_{*}^{-2} \sim \beta_{*}$. We can easily verify that as $X \uparrow 1$, (159) matches to (146) as $\alpha \rightarrow-\infty$. Note that in this limit we have

$$
\begin{aligned}
\int_{-\infty}^{\infty} & \frac{(u+\alpha)^{r-1}}{\left(u+\alpha+\beta_{*}^{2}\right)^{r+1}} e^{-u^{2} / 2} d u \\
& \sim \beta_{*}^{-2-2 r}(r-1) ! e^{-\alpha^{2} / 2}(-\alpha)^{-r} .
\end{aligned}
$$

For $\alpha \rightarrow+\infty$ we have

$$
\int_{-\alpha}^{\infty} \frac{(u+\alpha)^{r-1}}{\left(u+\alpha+\beta_{*}^{2}\right)^{r+1}} e^{-u^{2} / 2} d u \sim \frac{\sqrt{2 \pi}}{\alpha^{2}}=\frac{\sqrt{2 \pi}}{\sqrt{\rho} \alpha_{*}^{2}}
$$

and then (146) clearly matches to (160) and in fact contains the latter as a limiting case. When $r=0$ we have, for $0<X<$ 1 ,

$$
\pi(k, 0)-\frac{\rho^{k} e^{-\rho}}{k !} \sim \frac{-\sqrt{X}}{1-X} \frac{\otimes_{*}}{2 \pi \rho^{3 / 4} \beta_{*}} e^{\rho(-1+X-X \log X)} ;
$$

for $X-1=\alpha_{*} \rho^{-1 / 4}, \alpha_{*}>0$, we have

$$
\pi(k, 0)-\frac{\rho^{k} e^{-\rho}}{k !} \sim-\frac{\otimes_{*}}{\sqrt{2 \pi} \rho^{1 / 2}} \frac{\beta_{*}}{\alpha_{*}} ;
$$

and for $n=m-k=\sqrt{\rho}(\beta-\alpha)=\rho^{3 / 4}\left(\beta_{*}-\alpha_{*}\right)=O(1)$

$$
\pi(k, 0) \sim \frac{\otimes_{*}}{\sqrt{2 \pi} \rho^{3 / 4}} \beta_{*}(n+1) .
$$


We can again easily verify that (147) matches to (164) for $X \rightarrow$ 1 and $\alpha \rightarrow-\infty$ and to (165) for $\alpha \rightarrow+\infty$ and $\alpha_{*}=\alpha \rho^{-1 / 4} \rightarrow$ 0 . Recalling that $k=\rho X=\rho+\rho^{3 / 4} \alpha_{*}=\rho+\rho^{3 / 4} \beta_{*}-n$, we have

$$
\begin{aligned}
& \frac{\rho^{k} e^{-\rho}}{k !}-\frac{\otimes_{*}}{\sqrt{2 \pi} \rho^{1 / 2}} \frac{\beta_{*}}{k !}=\frac{\rho^{k} e^{-\rho}}{k !}-\frac{\otimes_{*}}{\sqrt{2 \pi \rho}} \frac{\beta_{*}}{\beta_{*}-\rho^{-3 / 4} n} \\
& =\frac{\otimes_{*}}{\sqrt{2 \pi \rho}}\left[\exp \left(\frac{n \beta_{*}}{\rho^{1 / 4}}\right)-1+O\left(\rho^{-3 / 4}\right)\right] \\
& \sim \frac{\otimes_{*}}{\sqrt{2 \pi} \rho^{3 / 4}} \beta_{*} n
\end{aligned}
$$

and this agrees with (166) for $n \rightarrow \infty$, which verifies the matching between (165) and (166). Thus we have given $\pi(k, r)$ for all ranges of $k$ for $r=O(1)$ and the scaling $m=\rho+\rho^{3 / 4} \beta_{*}$, $\beta_{*}>0$. However, only for the range $k=\rho+O(\sqrt{\rho})$ do we get the new results in (146) and (147).

Next we examine the corner $(X, Y)=\left(X_{0}, Y_{0}\right)$, so both primary and secondary spaces will be nearly full. For regions $\mathscr{R}_{3} \cup \mathscr{R}_{4}$ most of the mass is in the range. We will need to consider the scales $m-k, R-r=O(1)$ and also $m-k=$ $O\left(\rho^{1 / 3}\right)$. Consider $R-r=O\left(\rho^{2 / 3}\right)$.

Proposition 15. For $k=m-o(\rho)$ and $r=R-o(\rho)$ one uses the variables $m=m-k=\rho^{1 / 3} v_{1}$ and $p=R-r=\rho^{2 / 3} T_{1}$, and the expansions are as follows:

(i) $\left(X_{0}, Y_{0}\right) \in \mathscr{R}_{1} \cup \mathscr{R}_{2} \cup \mathscr{R}_{3} \cup \mathscr{R}_{4}, n=O(1), p=O(1)$

$$
\pi(k, r) \sim \frac{Q(0,0)}{X_{0}} \frac{1}{2 \pi i} \oint \frac{1-w}{z_{-}-w} z_{+}^{-n-1} w^{-p-1} d w
$$

where $z_{ \pm}(w)$ are defined in (50) and the expansions for $Q(0,0)$ are in Proposition 1; in particular for region $\mathscr{R}_{3} \cup \mathscr{R}_{4}$ one has $Q(0,0) \sim 1-X_{0}-Y_{0}>0$ and then (168) is a proper discrete distribution over the range(s) $n \geqslant 0, p \geqslant 0$.

(ii) $\left(X_{0}, Y_{0}\right) \in \mathscr{R}_{4}, m-k=\rho^{1 / 3} v_{1}=O\left(\rho^{1 / 3}\right), v_{1}>0$, $R-r=\rho^{2 / 3} T_{1}=O\left(\rho^{2 / 3}\right)$

$$
\begin{aligned}
\pi(k, r) \sim \rho^{-2 / 3}\left(1-X_{0}-Y_{0}\right) \\
\cdot \frac{\left(1-\sqrt{X_{0}}\right)^{5 / 3} X_{0}^{-1 / 6}}{\left(\sqrt{X_{0}}-X_{0}-Y_{0}\right)\left(W_{0}+Y_{0}\right)} \mathscr{F}(\nu, T)\left(\sqrt{X_{0}}\right)^{n} \\
\cdot\left(\frac{Y_{0}}{Y_{0}+W_{0}}\right)^{p} \\
\cdot \exp \left[-\frac{\rho^{1 / 3} W_{0}}{2 Y_{0}\left(W_{0}+Y_{0}\right)} T_{1}^{2}-\frac{W_{0}\left(W_{0}+2 Y_{0}\right)}{6 Y_{0}^{2}\left(W_{0}+Y_{0}\right)^{2}} T_{1}^{3}\right]
\end{aligned}
$$

where

$$
\begin{aligned}
\mathscr{F}(\nu, T) & =\frac{1}{2 \pi i} \frac{d}{d T}\left\{\int_{-i \infty}^{i \infty} \frac{A i(\nu+\theta)}{\theta A i(\theta)} e^{\theta T} d \theta\right\} \\
& =\sum_{j=0}^{\infty} \frac{A i\left(\nu+r_{j}\right)}{A i^{\prime}\left(r_{j}\right)} e^{r_{j} T}, \quad T>0, \\
\nu_{1} & =\nu\left(\frac{X_{0}}{1-\sqrt{X_{0}}}\right)^{1 / 3}, \\
T_{1} & =\frac{\left(W_{0}+Y_{0}\right) X_{0}^{1 / 6}}{\left(1-\sqrt{X_{0}}\right)^{2 / 3}} T,
\end{aligned}
$$

and $r_{j}$ are the roots of $A i(\cdot)$, ordered as $0>r_{0}>r_{1}>$ ...

(iii) $\left(X_{0}, Y_{0}\right) \in \mathscr{R}_{4}, m-k=n=O(1), R-r=p=\rho^{2 / 3} T_{1}=$ $O\left(\rho^{2 / 3}\right)$

$$
\begin{aligned}
\pi(k, r) \sim \rho^{-1}\left(1-X_{0}-Y_{0}\right) & \frac{W_{0} X_{0}^{-1 / 2}}{\left(\sqrt{X_{0}}-X_{0}-Y_{0}\right)\left(W_{0}+Y_{0}\right)}\left(\sqrt{X_{0}}\right)^{n} \\
\cdot & \left(\frac{Y_{0}}{Y_{0}+W_{0}}\right)^{p}\left[n+\frac{\sqrt{X_{0}}\left(Y_{0}+W_{0}\right)}{\left(Y_{0}+W_{0}\right) \sqrt{X_{0}}-Y_{0}}\right] \\
\cdot & {\left[\sum_{j=0}^{\infty} e^{r_{j} T}\right] \exp \left[-\rho^{1 / 3} \frac{W_{0}}{2 Y_{0}\left(W_{0}+Y_{0}\right)} T_{1}^{2}\right.} \\
- & \left.\frac{W_{0}\left(W_{0}+2 Y_{0}\right)}{6 Y_{0}^{2}\left(W_{0}+Y_{0}\right)^{2}} T_{1}^{3}\right], \quad T>0 .
\end{aligned}
$$

(iv) $\left(X_{0}, Y_{0}\right) \in \mathscr{R}_{3} \cap \mathscr{R}_{4}, m-k=n=\rho^{1 / 3} v_{1}=O\left(\rho^{1 / 3}\right)$, $v_{1} \geqslant 0, R-r=p=\rho^{2 / 3} T_{1}=O\left(\rho^{2 / 3}\right)$

$$
\begin{gathered}
\pi(k, r) \sim \rho^{-1 / 3}\left(1-\sqrt{X_{0}}\right)^{4 / 3} X_{0}^{-1 / 3}\left(\sqrt{X_{0}}\right)^{n} \\
\cdot\left(\frac{Y_{0}}{Y_{0}+W_{0}}\right)^{p} \exp \left[-\rho^{1 / 3} \frac{W_{0}}{2 Y_{0}\left(W_{0}+Y_{0}\right)} T_{1}^{2}\right. \\
\left.-\frac{W_{0}\left(W_{0}+2 Y_{0}\right)}{6 Y_{0}^{2}\left(W_{0}+Y_{0}\right)^{2}} T_{1}^{3}\right] \overline{\mathscr{F}}\left(\nu, T ; \delta_{1}\right),
\end{gathered}
$$

where

$$
\begin{aligned}
& \overline{\mathscr{F}}\left(\nu, T ; \delta_{1}\right)=\frac{1}{2 \pi i} \int_{-i \infty}^{i \infty} \frac{A i(\nu+\theta)}{[A i(\theta)]^{2}} e^{\theta\left(T-\delta_{1}\right)} \\
& \cdot\left(\int_{\theta}^{\infty} e^{\delta_{1} u} A i(u) d u\right) d \theta, \\
& X_{0}+Y_{0}=\sqrt{X_{0}}+\rho^{-1 / 3} \delta_{*}, \\
& \delta_{*}=X_{0}^{1 / 6}\left(1-\sqrt{X_{0}}\right)^{1 / 3} \delta_{1}
\end{aligned}
$$

and $(\nu, T)$ is given by (171) in terms of $\left(\nu_{1}, T_{1}\right)$. 
When $\left(X_{0}, Y_{0}\right) \in \mathscr{R}_{3} \cup \mathscr{R}_{4}$ most mass concentrates on the $(n, p)$ scale so there tend to be but a few available primary and secondary spaces. For $\left(X_{0}, Y_{0}\right) \in \mathscr{R}_{1} \cup \mathscr{R}_{2}\left(X_{0}+\right.$ $\left.Y_{0}>1\right)$, the result in (168) still applies but now $Q(0,0)$ is exponentially small (cf. (30)). For $X_{0}+Y_{0}-1=O\left(\rho^{-1 / 2}\right)$ we have $Q(0,0)=O\left(\rho^{-1 / 2}\right)$. Later we will study the behavior of the contour integral in (168) as $n$ and/or $p \rightarrow \infty$, and we will see that for $\mathscr{R}_{1} \cup \mathscr{R}_{2}$ we can get exponential growth in certain sectors, such as if $p \rightarrow \infty$ with $n=O(1)$. From Proposition 15 we also see that the probabilities of finding $O\left(\rho^{1 / 3}\right)$ empty primary spaces and $O\left(\rho^{2 / 3}\right)$ secondary spaces are quite complicated, and their estimation involves contour integrals of Airy functions. These probabilities are however quite small, in view of the factors $\left(\sqrt{X_{0}}\right)^{n}=\left(\sqrt{X_{0}}\right)^{\rho^{1 / 3} \gamma_{1}}$ and $\left[Y_{0} /\left(Y_{0}+W_{0}\right)\right]^{p}=\left[Y_{0} /\left(Y_{0}+W_{0}\right)\right]^{\rho^{2 / 3} T_{1}}$ in (169) and (173). In (173) we can use (175) and rewrite the result in terms of $X_{0}$ and $\delta_{*}$, thus eliminating $Y_{0}$.

Finally we give results that apply near the transition curves that separate $\mathscr{D}_{0}, \mathscr{D}_{-}$, and $\mathscr{D}_{+}$. Note $\mathscr{D}_{0}$ meets $\mathscr{D}_{+}$for region(s) $\mathscr{R}_{1} \cup \mathscr{R}_{2} \cup \mathscr{R}_{3}, \mathscr{D}_{+}$meets $\mathscr{D}_{-}$for region $\mathscr{R}_{2} \cup \mathscr{R}_{3}$, and $\mathscr{D}_{0}$ meets $\mathscr{D}_{-}$for region $\mathscr{R}_{4}$ (see also Figures 3-6).

Proposition 16. For $\left(X_{0}, Y_{0}\right) \in \mathscr{R}_{1} \cup \mathscr{R}_{2} \cup \mathscr{R}_{3}, Y-\widetilde{Y}(X) \equiv$ $\eta_{*} / \sqrt{\rho}=O\left(\rho^{-1 / 2}\right)$, and $0<X<X_{0}$ one has

$$
\begin{aligned}
& \pi(k, r) \sim C(\rho) K_{+}(X, \widetilde{Y}(X)) \\
& \quad \cdot \frac{1}{\sqrt{2 \pi}}\left(\int_{\eta_{*} / \sqrt{b\left(t_{*}\right)}}^{\infty} e^{-u^{2} / 2} d u\right) \exp \left[\rho \Psi_{+}(X, \widetilde{Y}(X))\right. \\
& \quad+\sqrt{\rho} \Psi_{+, Y}(X, \widetilde{Y}(X)) \eta_{*} \\
& \left.\quad+\frac{1}{2} \Psi_{+, Y Y}(X, \widetilde{Y}(X)) \eta_{*}^{2}\right],
\end{aligned}
$$

where

$$
\begin{aligned}
& \Psi_{+}(X, \widetilde{Y}(X))=X_{0} \log X_{0}+X_{0}+Y_{0}-1-2 X_{0} \log \left(X_{0}+Y_{0}\right)+\frac{Y_{0}}{X_{0}+Y_{0}}\left(1-e^{t_{*}}\right)-X \log \left(1-\frac{Y_{0} e^{t_{*}}}{X_{0}+Y_{0}}\right)-\widetilde{Y}(X) \\
& \cdot \log \left[1-\left(1-X_{0}-Y_{0}\right) e^{t_{*}}\right] \text {, } \\
& t_{*}=\log Y_{0}-\log \left[Y_{0}-\left(X_{0}+Y_{0}\right)\left(Y_{0}-\tilde{Y}(X)\right)\right] \text {, } \\
& \Psi_{+, Y}(X, \widetilde{Y}(X))=\log \left[\frac{Y_{0}-\left(X_{0}+Y_{0}\right)\left(Y_{0}-\tilde{Y}(X)\right)}{\left(X_{0}+Y_{0}\right) \tilde{Y}(X)}\right], \\
& \Psi_{+, Y Y}(X, \widetilde{Y}(X))=-e^{t_{*}} \frac{s_{Y}^{0}-\left(1-X_{0}-Y_{0}\right) t_{Y}^{0}}{1-\left(1-X_{0}-Y_{0}\right) e^{t_{*}}}, \\
& s_{Y}^{0}=\frac{\left(X_{0}+Y_{0}\right)\left(X_{0}+Y_{0}+Y_{0} e^{-t_{*}} t_{Y}^{0}\right)}{\left(X_{0}+Y_{0}\right)^{2}+\left(e^{-t_{*}}-1\right) X_{0}}, \\
& t_{Y}^{0}=e^{t_{*}}\left(1-e^{t_{*}}\right)\left(X_{0}+Y_{0}\right)\left[X_{0} e^{t_{*}}+\left(X_{0}+Y_{0}\right)^{2}\right] \\
& \cdot\left\{\left(X_{0}+Y_{0}\right)\left(X_{0}^{2}-Y_{0}^{2}-X_{0}\right)+e^{t_{*}}\left[X_{0}-\left(X_{0}+Y_{0}\right)^{2}\right]^{2}+e^{2 t_{*}}\left(2-e^{t_{*}}\right) X_{0} Y_{0}+e^{3 t_{*}} Y_{0}\left(X+Y_{0}\right)^{2}\right\}^{-1}, \\
& K_{+}(X, \tilde{Y}(X))=\frac{Y_{0} \sqrt{X_{0}}\left[\left(X_{0}+Y_{0}\right)^{2}-X_{0}\right]^{3 / 2}}{\sqrt{2 \pi}\left|\Delta_{+}^{0}\right|^{1 / 2}\left(X_{0}+Y_{0}\right)^{3} \sqrt{\tilde{Y}(X)} \sqrt{X_{0} Y_{0}-\left(X_{0}+Y_{0}\right)^{2}\left(Y_{0}-\tilde{Y}(X)\right)}}, \\
& b\left(t_{*}\right)=\frac{X_{0} Y_{0}\left|\Delta^{0}\right|\left|\Delta_{+}^{0}\right|}{\left(X_{0}+Y_{0}\right)\left[\left(X_{0}+Y_{0}\right)^{2}-X_{0}\right]}\left[\left(1-X_{0}-Y_{0}\right) e^{-t_{*}}-\frac{Y_{0} e^{t_{*}}}{X_{0}+Y_{0}}\right]^{-2} \text {, } \\
& \Delta_{+}^{0}=\Delta_{+}^{0}(X)=\frac{1}{1-E_{*}}\left[D_{*}\left(E_{*}-D_{*}\right) e^{t_{*}}-2 D_{*}\left(1-D_{*}\right)-\left(D_{*}-E_{*}\right)^{2} e^{-t_{*}}+\left(D_{*}+E_{*}-2 D_{*} E_{*}\right) e^{-2 t_{*}}\right], \\
& D_{*}=\frac{Y_{0}}{X_{0}+Y_{0}} \\
& E_{*}=1-X_{0}-Y_{0} \\
& \Delta^{0}=\Delta^{0}(X)=\left(e^{-t_{*}}-1\right)\left[\frac{Y_{0}}{X_{0}+Y_{0}}+\frac{Y_{0}\left(\left(X_{0}+Y_{0}\right)^{2}-X_{0}\right)}{X_{0}\left(X_{0}+Y_{0}\right)} e^{t_{*}}+e^{-t_{*}}\left(1-2\left(X_{0}+Y_{0}\right)+\left(X_{0}+Y_{0}\right)^{3} X_{0}^{-1}\right)\right] \text {. }
\end{aligned}
$$


$\widetilde{Y}(X)$ is given by (56), and $C(\rho)$ has the expansions in (31)-(33) for the different regions $\mathscr{R}_{j}$.

For $\eta_{*} \rightarrow-\infty$ we are moving into region $\mathscr{D}_{+}$and the integral in (176) approaches $\sqrt{2 \pi}$, and then (176) becomes simply the expansion of the $\mathscr{D}_{+}$result in (60), about $\mathrm{Y}=$ $\tilde{Y}(X)$. Thus the matching of (176) to $\mathscr{D}_{+}$is immediate, and we can also verify the matching to (65), by expanding the latter as $Y \downarrow \tilde{Y}(X)$, and (176) for $\eta_{*} \rightarrow+\infty$. We recall that Proposition 2 shows that the expansion of $C_{0}(\rho) / C(\rho)$ is the same for regions $\mathscr{R}_{1}, \mathscr{R}_{2}$, and $\mathscr{R}_{3}$. The function $t_{*}=t_{*}(X)$ in (178) is obtained by setting $s=Y_{0}$ and then $Y=\tilde{Y}(X)$ in (62). The function $\Delta_{+}^{0}(X)$ is obtained by setting $s=Y_{0}$ and $t=t_{*}(X)$ in (64) and corresponds to the values of this Jacobian along the curve $Y=\widetilde{Y}(X)$. Note also that $t_{*}(X)$ can be obtained by setting $A=Y_{0} /\left(X_{0}+Y_{0}\right)$ and $Y=\widetilde{Y}(X)$ in (68), and $\Delta^{0}(X)$ corresponds to the Jacobian in (71) along the curve $Y=\tilde{Y}(X)$, with $\tau=t_{*}(X)$.

Proposition 17. For $\left(X_{0}, Y_{0}\right) \in \mathscr{R}_{2} \cup \mathscr{R}_{3}, Y-Y_{*}(X)=$ $\rho^{-1 / 3} \xi_{1}=O\left(\rho^{-1 / 3}\right)$, and $0<X<X_{0}$ one has

$$
\begin{aligned}
& \pi(k, r) \sim \rho^{-1 / 2} C(\rho) \exp \left[\rho \Phi\left(X, Y_{*}(X)+\rho^{-1 / 3} \xi_{1}\right)\right] \\
& \cdot \frac{1}{2 \pi} \frac{W_{0}}{Y_{*}(X)} \frac{1}{\sqrt{W_{0}-\left(W_{0}+Y_{*}(X)\right)^{2}}} \frac{1}{2 \pi i} \\
& \cdot \int_{-i \infty}^{i \infty} \frac{1}{[A i(\theta)]^{2}} \exp \left[-\frac{\left(1-\sqrt{X_{0}}\right)^{2 / 3}}{X_{0}^{1 / 6}\left(W_{0}+Y_{*}(X)\right)}\right. \\
& \left.\cdot \xi_{1} \theta\right] d \theta,
\end{aligned}
$$

where

$$
\begin{aligned}
& \rho \Phi\left(X, Y_{*}+\rho^{-1 / 3} \xi_{1}\right) \\
& =\rho \Phi\left(X, Y_{*}\right)+\rho^{2 / 3} \Phi_{Y}\left(X, Y_{*}\right) \xi_{1} \\
& \quad+\frac{1}{2} \rho^{1 / 3} \Phi_{Y Y}\left(X, Y_{*}\right) \xi_{1}^{2}+\frac{1}{6} \Phi_{Y Y Y}\left(X, Y_{*}\right) \xi_{1}^{3} \\
& \quad+o(1), \\
& \Phi\left(X, Y_{*}\right) \\
& =Y_{*} \log \left(1+\frac{W_{0}}{Y_{*}}\right)-\frac{W_{0}}{W_{0}+Y_{*}} \\
& \quad-X \log \left(\frac{Y_{*}}{W_{0}+Y_{*}}\right) \\
& \Phi_{Y}\left(X, Y_{*}\right)=\log \left(1+\frac{W_{0}}{Y_{*}}\right)
\end{aligned}
$$

$$
\begin{aligned}
& \Phi_{Y Y}\left(X, Y_{*}\right)=-\frac{W_{0}}{Y_{*}\left(W_{0}+Y_{*}\right)}, \\
& \Phi_{Y Y Y}\left(X, Y_{*}\right)=\frac{W_{0}\left(W_{0}+2 Y_{*}\right)}{Y_{*}^{2}\left(W_{0}+Y_{*}\right)^{2}},
\end{aligned}
$$

and $C(\rho)$ has the expansions in (31)-(33) (one can replace $\rho^{-1 / 2} C(\rho)$ by $\rho^{-1 / 3} C_{1}(\rho)$ in (188)), and $Y_{*}=Y_{*}(X)=$ $Y_{*}\left(X ; X_{0}\right)$ is given in $(75)$.

For $\xi_{1} \rightarrow+\infty$ we can expand the integral in (188) by the saddle point method, after shifting the integration contour far to the right, with $\operatorname{Re}(\theta) \gg 1$. Then the Airy function in the integrand may be approximated using

$$
\operatorname{Ai}(z) \sim \frac{1}{2 \sqrt{\pi}} z^{-1 / 4} e^{-(2 / 3) z^{3 / 2}}
$$

$$
z \longrightarrow \infty,|\arg z|<\pi
$$

and we can verify that (188) for $\xi_{1} \rightarrow+\infty$ matches to the $\mathscr{D}_{+}$ result in (60), as $Y \downarrow Y_{*}(X)$. For $\xi_{1} \rightarrow-\infty$ the behavior of the integral in (188) is determined by the singularity with the largest real part, which is the double pole at $\theta=r_{0}(<0)$. Then standard singularity analysis can be used to show that (188) for $\xi_{1} \rightarrow-\infty$ agrees with the expansion of (79) as $Y \uparrow$ $Y_{*}(X)$. Note that in this limit $\rho^{1 / 3} \Phi_{*}\left(s_{1}\right)$ becomes $O(1)$ and proportional to $\xi_{1}$.

Proposition 18. For $\left(X_{0}, Y_{0}\right) \in \mathscr{R}_{4}, Y-Y_{c}(X)=\rho^{-1 / 3} \eta_{* *}=$ $O\left(\rho^{-1 / 3}\right)$, and $X_{L}<X<X_{0}$ (with $X_{L}$ defined in item (vii) of Proposition 8) one has

$$
\pi(k, r) \sim \frac{1-X_{0}-Y_{0}}{\rho^{5 / 6} \sqrt{2 \pi}} \frac{\left(1-\sqrt{X_{0}}\right)^{4 / 3} X_{0}^{1 / 6}}{\sqrt{X_{0}}-X_{0}-Y_{0}}
$$

$$
\begin{aligned}
& \cdot \sqrt{\frac{Y_{0}}{Y_{c}(X)}} \sqrt{\frac{W_{0}+Y_{0}}{Y_{0}-Y_{c}(X)}}\left\{\left[2 W_{0}+Y_{0}+Y_{c}(X)\right]\right. \\
& \left.\cdot\left[W_{0}+Y_{c}(X)-\sqrt{W_{0}}\left(W_{0}+Y_{0}\right)\right]\right\}^{-1 / 2} \\
& \cdot \exp \left\{\rho\left[\Phi\left(X, Y_{c}(X)+\rho^{-1 / 3} \eta_{* *}\right)-\Phi\left(X_{0}, Y_{0}\right)\right]\right\} \\
& \cdot \frac{1}{2 \pi i} \int_{-i \infty}^{i \infty} \frac{1}{A i(\theta)} \exp \left[-\frac{\left(1-\sqrt{X_{0}}\right)^{2 / 3}}{X_{0}^{1 / 6}\left(W_{0}+Y_{0}(X)\right)}\right. \\
& \left.\cdot \eta_{* *} \theta\right] d \theta,
\end{aligned}
$$

where $Y_{c}(X)=Y_{c}\left(X ; X_{0}, Y_{0}\right)$ is defined in (86).

Thus the transition from $\mathscr{D}_{0}$ to $\mathscr{D}_{-}$involves a slightly different integral (cf. (191)) compared to the transition from $\mathscr{D}_{+}$to $\mathscr{D}_{-}$(cf. (188)), as the former has simple poles at the 
Airy roots. Using standard asymptotic analysis we can show that

$$
\begin{aligned}
& \frac{1}{2 \pi i} \int_{-i \infty}^{i \infty} \frac{e^{-\star \theta}}{\operatorname{Ai}(\theta)} d \theta \sim 2 \star e^{-\star^{3} / 3}, \quad \star \longrightarrow+\infty, \\
& \frac{1}{2 \pi i} \int_{-i \infty}^{i \infty} \frac{e^{-\star \theta}}{\operatorname{Ai}(\theta)} d \theta \sim \frac{e^{-\star r_{0}}}{\operatorname{Ai}^{\prime}\left(r_{0}\right)}, \quad \star \longrightarrow-\infty
\end{aligned}
$$

and (192) can be used to verify the matching between (191) and the $\mathscr{D}_{0}$ result in (65), where $C_{0} \sim \rho^{-1 / 2}\left(1-X_{0}-Y_{0}\right)$ in $\mathscr{R}_{4}$. Similarly (193) can be used to verify matching to the $\mathscr{D}_{-}$ result in (79), with $C_{1}$ now given by (41). The factor involving $\rho \Phi\left(X, Y_{c}+\rho^{-1 / 3} \eta_{* *}\right)$ in (191) can be expanded in Taylor series, similarly to (189), by replacing $Y_{*}(X)$ by $Y_{c}(X)$ and $\xi_{1}$ by $\eta_{* *}$.

As we approach $\mathscr{R}_{3} \cap \mathscr{R}_{4}$ (where $X_{0}+Y_{0}=\sqrt{X_{0}}$ ) from within $\mathscr{R}_{4}$ we see that (191) develops a singularity, in view of the factor $\left(\sqrt{X_{0}}-X_{0}-Y_{0}\right)^{-1}$. As we approach $\mathscr{R}_{3} \cap \mathscr{R}_{4}$ from within $\mathscr{R}_{3}$, the expression for $K_{+}(X, \widetilde{Y}(X))$ in (183) vanishes and that for $b\left(t_{*}\right)$ in (184) develops a singularity. Thus the expansions in both Propositions 16 and 18 become invalid, and below we give a new result that applies for $\mathscr{R}_{3} \cap \mathscr{R}_{4}$, where $\mathscr{D}_{0}$ meets $\mathscr{D}_{-}$.

Proposition 19. For $\left(X_{0}, Y_{0}\right) \in \mathscr{R}_{3} \cap \mathscr{R}_{4}$, with $X_{0}+Y_{0}=$ $\sqrt{X_{0}}+\delta_{*} \rho^{-1 / 3}$ and $\delta_{*}=O(1)$,

$$
\begin{aligned}
\pi(k, r) \sim & \frac{\left(1-\sqrt{X_{0}}\right)^{3} X_{0}^{1 / 4} \exp \left\{\rho\left[\Phi\left(X, Y_{c}(X)+\rho^{-1 / 3} \eta_{* *}\right)-\Phi\left(X_{0}, Y_{0}\right)\right]\right\}}{\sqrt{2 \pi \rho} Y_{*}(X) \sqrt{\sqrt{X_{0}}-X_{0}-Y_{*}(X)} \sqrt{Y_{*}(X)+\left(1-\sqrt{X_{0}}\right)\left(2-\sqrt{X_{0}}\right)} \frac{1}{2 \pi i}} \\
& \cdot \int_{-i \infty}^{i \infty} \frac{e^{-\theta \delta_{1}} \int_{\theta}^{\infty} e^{\delta_{1} u} \operatorname{Ai}(u) d u}{[\operatorname{Ai}(\theta)]^{2}} \exp \left[-\frac{\left(1-\sqrt{X_{0}}\right)^{2 / 3}}{X_{0}^{1 / 6}\left(W_{0}+Y_{*}(X)\right)} \eta_{* *} \theta\right] d \theta,
\end{aligned}
$$

where $\delta_{1}=X_{0}^{-1 / 6}\left(1-\sqrt{X_{0}}\right)^{-1 / 3} \delta_{*}$ and $Y_{c}(X)$ is in (86) and $Y_{*}(X)$ in (75).

With the scaling $X_{0}+Y_{0}-\sqrt{X_{0}}=O\left(\rho^{-1 / 3}\right)$, the curves $Y_{c}(X), Y_{*}(X)$, and $\widetilde{Y}(X)$ nearly coincide, with the differences being $O\left(\rho^{-1 / 3}\right)$. In (194) we can again replace $\Phi\left(X, Y_{c}+\right.$ $\left.\rho^{-1 / 3} \eta_{* *}\right)$ by its Taylor expansion and replace $Y_{0}$ everywhere by $\sqrt{X_{0}}-X_{0}+\delta_{*} \rho^{-1 / 3}$, thus writing the result in terms of $X_{0}$ and $\delta_{*}\left(\right.$ or $\left.\delta_{1}\right)$, along with $X$ and $\eta_{* *}$. Note that $\eta_{* *}$ is still defined by $\eta_{* *}=\rho^{1 / 3}\left[Y-Y_{c}(X)\right]$, which differs from $\rho^{1 / 3}\left[Y-Y_{*}(X)\right]$ by an amount that is $O(1)$. Thus, for region $\mathscr{R}_{3} \cap \mathscr{R}_{4}$ the transition range in state space, between $\mathscr{D}_{-}$ and $\mathscr{D}_{0}$, involves a somewhat more complicated integral than those in Propositions 18 and 19.

This completes our summary and discussion of the various regions of state space, which carry zero volume in $(X, Y)$ space but are necessary since the results in $\mathscr{D}_{0}, \mathscr{D}_{+}$, and $\mathscr{D}_{-}$do not always apply.

\section{Asymptotic Expansion in Region $\mathscr{D}_{0}$}

In this section we will construct the expansion for $(X, Y) \in$ $\mathscr{D}_{0}$, that is, (65). The analysis for the complementary regions $\mathscr{D}_{+}$and $\mathscr{D}_{-}$is virtually identical to that for the model with an infinite secondary capacity $(R=\infty)$, and the detailed calculations can be found in [10]. Here we will only discuss, for regions $\mathscr{D}_{+}$and $\mathscr{D}_{-}$, those aspects that change when $R<$ $\infty$. In order to uniquely determine the expansion in $\mathscr{D}_{0}$, we will need to use asymptotic matching to the corner expansion that applies on the scale $k=m-O(1), r=R-O(1)$, and this is discussed in Section 4.2. For parameter region $\mathscr{R}_{4}$ (and also for $\mathscr{R}_{3} \cap \mathscr{R}_{4}$ ) we will also need to carefully analyze the scale $k=m-O\left(\rho^{1 / 3}\right)$ and $r=R-O\left(\rho^{2 / 3}\right)$, which will be necessary to determine the multiplicative constant $C_{1}=C_{1}\left(\rho ; X_{0}, Y_{0}\right)$ that arises in the asymptotic expansion in $\mathscr{D}_{-}$; this analysis is done in Section 4.3.

4.1. Ray Expansion in the Interior of $\mathscr{D}_{0}$. We analyze the scaled equation in (17) using the ray method of geometrical optics, where we assume an expansion of the form

$$
\begin{aligned}
& P(X, Y)=C_{0}(\rho) e^{\rho \Psi(X, Y)}\left[K(X, Y)+\rho^{-1} K^{(1)}(X, Y)\right. \\
& \left.+\rho^{-2} K^{(2)}(X, Y)+\cdots\right] .
\end{aligned}
$$

Then we have

$$
\begin{aligned}
& \frac{P\left(X \pm \rho^{-1}, Y\right)}{P(X, Y)}=e^{ \pm \Psi_{X}(X, Y)}[1 \\
& \left.+\rho^{-1}\left(\frac{ \pm K_{X}}{K}+\frac{1}{2} \Psi_{X X}(X, Y)\right)+O\left(\rho^{-2}\right)\right]
\end{aligned}
$$

and using (195) in (17) we obtain in the limit as $\rho \rightarrow \infty$ the “eikonal" equation

$$
1+X+Y=Y e^{\Psi_{Y}}+X e^{\Psi_{X}}+e^{-\Psi_{X}}
$$

and at the next order in $\rho^{-1}$ the "transport" equation

$$
\begin{aligned}
e^{\Psi}\left(Y K_{Y}+\frac{1}{2} Y \Psi_{Y Y} K+K\right) \\
+e^{\Psi_{X}}\left(X K_{X}+\frac{1}{2} X \Psi_{X X} K+K\right) \\
+e^{-\Psi_{X}}\left(-K_{X}+\frac{1}{2} \Psi_{X X} K\right)=0 .
\end{aligned}
$$


The first-order PDE in (197) can be solved by the method of characteristics (see [14]), where one must solve the five ODEs

$$
\begin{aligned}
\frac{d X}{d \tau} & =e^{-\Psi_{X}}-X e^{\Psi_{X}}, \\
\frac{d Y}{d \tau} & =-Y e^{\Psi_{Y}}, \\
\frac{d \Psi}{d t} & =\Psi_{X} \frac{d X}{d \tau}+\Psi_{Y} \frac{d Y}{d \tau} \\
& =\Psi_{X}\left(e^{-\Psi_{X}}-X e^{\Psi_{X}}\right)-Y \Psi_{Y} e^{\Psi_{Y}}, \\
\frac{d \Psi_{X}}{d \tau} & =e^{\Psi_{X}}-1, \\
\frac{d \Psi_{Y}}{d \tau} & =e^{\Psi_{Y}}-1 .
\end{aligned}
$$

Here $\tau$ is a parameter along a given characteristic curve, which is also called a "ray," due to applications in optics.

To uniquely specify the solution $\Psi(X, Y)$ to (197), we must either specify $\Psi$ along some curve, called the "initial manifold," in the $(X, Y)$ plane, or use a singular solution, where all the rays emanate from a single point. The appropriate solution to the eikonal equation must be determined for each individual problem, and we will see that for the present model we will need to use three different solutions $\Psi$ to (197), with two having the boundary $X=X_{0}$ as the initial manifold and the third corresponding to all the rays emanating from the corner point $\left(X_{0}, Y_{0}\right)$. The first two solutions will correspond to regions $\mathscr{D}_{+}$and $\mathscr{D}_{-}$and the third to $\mathscr{D}_{0}$. Since $\mathscr{D}_{+}$and $\mathscr{D}_{-}$ arose also in the infinite capacity model, where $R=\infty$, we discuss these only briefly, and the details of the corresponding solutions to (197)-(201) can be found in [10].

Let us denote the solution in region $\mathscr{D}_{+}$as $C(\rho) K_{+}(X, Y) e^{\rho \Psi_{+}(X, Y)}$ to distinguish it from (195), which will apply in $\mathscr{D}_{0}$. If this expansion will satisfy the boundary equation along $X=X_{0}$ (or $k=m$ ) in (4), or, equivalently, (18), we must have

$$
X_{0} e^{\Psi_{+, X}\left(X_{0}, Y\right)}=e^{-\Psi_{+, Y}\left(X_{0}, Y\right)} .
$$

Requiring $\Psi_{+}(X, Y)$ to satisfy (202) is equivalent, up to an additive constant in $\Psi_{+}$which can be incorporated into $C(\rho)$, to specifying $\Psi_{+}$along the initial manifold $X=X_{0}$. Solving (199)-(201) subject to (202) leads to

$$
\begin{aligned}
& \Psi_{+, X}=-\log \left(1-\frac{s}{s+X_{0}} e^{t}\right), \\
& \Psi_{+, Y}=-\log \left[1+\left(s+X_{0}-1\right) e^{t}\right]
\end{aligned}
$$

and then the rays are given in parametric form by (62), which relates $(s, t)$ to $(X, Y)$. When $t=0$ we have $(X, Y)=\left(X_{0}, s\right)$ so that $s$ is the point where a ray hits the line $X=X_{0}$. We use now $t$ instead of $\tau$ as the parameter along a given ray, and $s$ is used to index the family. Finally, solving (200) with $(\Psi, \tau)$ replaced by $\left(\Psi_{+}, t\right)$ leads to the expression in (61). We also note that when $t=0\left(X=X_{0}\right)$ we have

$$
\Psi_{+}\left(X_{0}, Y\right)=X_{0}-1+Y-\left(X_{0}+Y\right) \log \left(X_{0}+Y\right)
$$

which is an explicit function of $Y$. From the first expression in (62) we find that for $t \rightarrow 0$

$$
X_{0}-X=\frac{\left(Y+X_{0}\right)^{2}-X_{0}}{Y+X_{0}} t+O\left(t^{2}\right) .
$$

It follows that for $t>0$ the rays that start from $X=X_{0}$ enter the state space, where $X \leqslant X_{0}$, for $t>0$ only if $\left(Y+X_{0}\right)^{2}-X_{0}>$ 0 , or $Y+X_{0}>\sqrt{X_{0}}$. If $X_{0}>1$ this condition holds for all $Y \geqslant 0$ and hence the rays fill the region $\mathscr{D}_{+}$indicated in Figure 3 . If $X_{0}<1$ and $Y_{0}+X_{0}>\sqrt{X_{0}}$ then the condition holds only for $Y$ in the interval $\sqrt{X_{0}}-X_{0}<Y<Y_{0}$. Then these rays fill the domain $\mathscr{D}_{+}$indicated in Figures 4 and 5. But if $X_{0}<1$ and $X_{0}+Y_{0}<\sqrt{X_{0}}$, which is true for parameter region $\mathscr{R}_{4}$, the condition never holds and then this ray expansion plays no role in the analysis (see also Figure 6). Once we compute $\Psi_{+}$in $\mathscr{D}_{+}$, we can integrate (198) to obtain $K_{+}(X, Y)$ in (63). Thus we have shown that (63), up to the constant $C(\rho)$ which we have yet to determine, holds in the portion $\mathscr{D}_{+}$of the state space, for parameter regions $\mathscr{R}_{1} \cup \mathscr{R}_{2} \cup \mathscr{R}_{3}$.

Now we observe that if $X_{0}>1, \Psi_{+}\left(X_{0}, Y\right)$ is maximal at $Y=0$ and by evaluating also $K_{+}$at $X=X_{0}$ and $Y=0$ we find that near the corner $(X, Y)=\left(X_{0}, 0\right)$ with $X=X_{0}(k=m)$ and $Y=r / \rho$, and we have

$$
C K_{+} e^{\rho \Psi_{+}} \sim C \frac{1}{\sqrt{2 \pi}} \frac{X_{0}-1}{X_{0}^{3 / 2}} X_{0}^{-r} e^{\rho\left(X_{0}-1-X_{0} \log X_{0}\right)} .
$$

The scale $r=O(1)$ for $X_{0}>1$ must be analyzed separately and the details are carried out in [10]. For $r=O(1)$ and $m-k=$ $n=O(1)$ the result in (141) applies, and by asymptotically matching this to (206) (for $r \geqslant 1$ ) we conclude that

$$
\begin{aligned}
C(\rho) & \sim \rho^{-1 / 2}, \\
\left(X_{0}, Y_{0}\right) & \in \mathscr{R}_{1} .
\end{aligned}
$$

If $X_{0}<1$ and $X_{0}+Y_{0}>1$ then $\Psi_{+}\left(X_{0}, Y\right)$ in (204) is maximal at $Y=1-X_{0}$, which lies in the range $\left(0, Y_{0}\right)$ precisely for parameter region $\mathscr{R}_{2}$. Expanding $\Psi_{+}(X, Y)$ in (61) about $X=$ $X_{0}$ and $Y=1-X_{0}$ leads to

$$
\begin{aligned}
& C K_{+} e^{\rho \Psi_{+}} \\
& \quad \sim C\left(1-X_{0}\right) X_{0}^{n} \frac{1}{\sqrt{2 \pi}} \exp \left\{-\frac{1}{2 \rho}[r-(\rho-m)]^{2}\right\} .
\end{aligned}
$$

For parameter region $\mathscr{R}_{2}$ the expansions in $\mathscr{D}_{-}$and $\mathscr{D}_{0}$ will be uniformly exponentially small in $\rho$, as there is little mass in these state space ranges. Thus the main contribution to the double sum in the normalization condition (10) will come from $\mathscr{D}_{+}$and in particular from the scale $m-k=n=$ $O(1)$ and $r=\rho-m+O(\sqrt{\rho})$ (corresponding to $Y=1-$ $\left.X_{0}+O\left(\rho^{-1 / 2}\right)\right)$. Then normalizing the approximation in (208), after approximating the sum over $r$ by an integral over $Y$, we conclude that $C(\rho) \sim \rho^{-1 / 2}$, and hence (207) applies also for parameter region $\mathscr{R}_{2}$ (and, by continuity, the relation holds in $\mathscr{R}_{1} \cap \mathscr{R}_{2}\left(X_{0}-1=O\left(\rho^{-1 / 2}\right)\right)$ also $)$.

We have thus shown that the ray expansion in $\mathscr{D}_{+}$has the state space regions $\mathscr{D}_{-}$and $\mathscr{D}_{0}$ as "shadows," and thus the 
other solutions to (197) must apply. To construct the solution in $\mathscr{D}_{\text {_ }}$ we must slightly modify the ansatz in (195) and now expand the joint distribution as

$$
\begin{gathered}
P(X, Y)=C_{1}(\rho) e^{\rho \Phi(X, Y)} e^{\rho^{1 / 3} \Phi_{1}(X, Y)}[L(X, Y) \\
\left.\quad+\rho^{-1 / 3} L^{(1)}(X, Y)+\rho^{-2 / 3} L^{(2)}(X, Y)+\cdots\right] .
\end{gathered}
$$

Now $\Phi$ will satisfy (197) and $L$ will satisfy (198), and the subexponential term $\Phi_{1}$ will satisfy the PDE

$$
Y e^{\Phi_{Y}} \Phi_{1, Y}+\left(X e^{\Phi_{X}}-e^{-\Phi_{X}}\right) \Phi_{1, X}=0 .
$$

Again the detailed analysis can be found in [10]. We now find that

$$
\begin{aligned}
& \Phi_{X}=-\log \left[1-\left(1-\sqrt{X_{0}}\right) e^{t_{1}}\right] \\
& \Phi_{Y}=-\log \left[1-\frac{W_{0}}{W_{0}+s_{1}} e^{t_{1}}\right]
\end{aligned}
$$

and the rays are given in parametric form by

$$
\begin{aligned}
& X=\left[1-\left(1-\sqrt{X_{0}}\right) e^{t_{1}}\right]\left[1-\left(1-\sqrt{X_{0}}\right) e^{-t_{1}}\right], \\
& Y=-W_{0}+\left(W_{0}+s_{1}\right) e^{-t_{1}},
\end{aligned}
$$

where we recall that $W_{0}=\left(1-\sqrt{X_{0}}\right)^{2}$. When $t_{1}=0$ we have $X=X_{0}$ and $\mathrm{Y}=s_{1}$, but unlike the rays in $\mathscr{D}_{+}$, those in $\mathscr{D}_{-}$ are all tangent to the boundary $X=X_{0}$. Thus the boundary is a "caustic boundary," for $0<Y<\sqrt{X_{0}}-X_{0}$ for region $\mathscr{R}_{2} \cup \mathscr{R}_{3}$ and for all $Y$ with $0<Y<Y_{0}$ for region $\mathscr{R}_{4}$. The solution $\Phi$ to (197) is now given by

$$
\begin{aligned}
\Phi(X, Y)=[ & \left.W_{0}-\left(W_{0}+s_{1}\right) e^{-t_{1}}\right] \log \left[1-\frac{W_{0} e^{t_{1}}}{W_{0}+s_{1}}\right] \\
& -\left(1-\sqrt{X_{0}}-e^{-t_{1}}\right)\left(1-\sqrt{X_{0}}-e^{t_{1}}\right) \\
& \cdot \log \left(1-\sqrt{W_{0}} e^{t_{1}}\right)-\sqrt{W_{0}} e^{t_{1}}+W_{0} \\
& \cdot \log \left(W_{0}+s_{1}\right)-\frac{1}{2} W_{0} \log W_{0} .
\end{aligned}
$$

For region $\mathscr{D}_{-}$we can invert transformation (212) and write $s_{1}$ and $t_{1}$ explicitly in terms of $X$ and $Y$. This leads to the expression in (81) for $s_{1}=s_{1}(X, Y)$, and then (80) gives $\Phi$ in terms of $X$ and $Y$.

Equation (210) implies that $\Phi_{1}(X, Y)$ is constant along a caustic ray, so we write $\Phi_{1}(X, Y)=\Phi_{*}\left(s_{1}\right)$. To determine $\Phi_{*}(\cdot)$ and also completely determine $L(X, Y)$ in (209), we need to construct two "nested" boundary layer corrections to the expansion in (209), near $X=X_{0}$, corresponding to the scales $X_{0}-X=O\left(\rho^{-2 / 3}\right)\left(m-k=O\left(\rho^{1 / 3}\right)\right)$ and $X_{0}-X=O\left(\rho^{-1}\right)(m-k=O(1))$. The boundary condition in (18) can only be imposed on the expansion that applies for $n=m-k=O(1)$. Using asymptotic matching between the expansions for $X_{0}-X=O\left(\rho^{-1}\right), X_{0}-X=O\left(\rho^{-2 / 3}\right)$, and the ray expansion in (209) allows us to determine (209) up to the multiplicative constant $C_{1}$.

To determine $C_{1}$ we need to, for regions $\mathscr{R}_{2}$ and $\mathscr{R}_{3}$, first relate $C_{1}(\rho)$ to the constant $C(\rho)$ in the $\mathscr{D}_{+}$expansion. This can be done by analyzing the transition curve $Y=Y_{*}(X)$, with the scaling $Y-Y_{*}(X)=O\left(\rho^{-1 / 3}\right)$. The details are again presented in [10], and this leads to the conclusion that

$$
\frac{C_{1}(\rho)}{C(\rho)} \sim \rho^{-1 / 6}, \quad\left(X_{0}, Y_{0}\right) \in \mathscr{R}_{2} \cup \mathscr{R}_{3} .
$$

In $\mathscr{R}_{2}$ we have $C(\rho) \sim \rho^{-1 / 2}$ so that $C_{1}(\rho) \sim \rho^{-2 / 3}$, but we have yet to determine either $C$ or $C_{1}$ for region $\mathscr{R}_{3}$. The conclusion in (214) can also be reached by constructing an expansion near the point $(X, Y)=\left(X_{0}, \sqrt{X_{0}}-X_{0}\right)$, with the scaling $X_{0}-X=O\left(\rho^{-2 / 3}\right)$ and $Y-\left(\sqrt{X_{0}}-X_{0}\right)=O\left(\rho^{-1 / 3}\right)$ (see subsection 5.3 in [10] for that analysis). Note also that the curve $Y=Y_{*}(X)$ corresponds to the ray with $s_{1}=\sqrt{X_{0}}-X_{0}$ in (212) and also the ray with $s=\sqrt{X_{0}}-X_{0}$ in (62). Then we have $\Psi_{+}\left(X, Y_{*}(X)\right)=\Phi\left(X, Y_{*}(X)\right)$ so that the exponential factors in the expansions in (60) and (209) are continuous along $Y=Y_{*}(X)$, which separates $\mathscr{D}_{+}$from $\mathscr{D}_{-}$.

The region $\mathscr{D}_{0}$ is a shadow of both the $\mathscr{D}_{+}$and $\mathscr{D}_{-}$rays for parameter regions $\mathscr{R}_{2} \cup \mathscr{R}_{3}$ and a shadow of the $\mathscr{D}_{-}$rays for parameter region $\mathscr{R}_{4}$. For $\mathscr{R}_{4}$, the caustic rays that fill $\mathscr{D}_{-}$ correspond to $0<s_{1}<Y_{0}$ in (212), and $s_{1}=Y_{0}$ corresponds to the curve $Y=Y_{c}(X)$ in (86), which separates $\mathscr{D}_{-}$from $\mathscr{D}_{0}$. To fill the shadow $\mathscr{D}_{0}$ and thus obtain an approximation to $P(X, Y)$ for $(X, Y) \in \mathscr{D}_{0}$, we must use a singular solution to (197), one that has all rays start from the corner point $(X, Y)=$ $\left(X_{0}, Y_{0}\right)$. To construct this solution we first integrate the two ODEs in (201), which yields

$$
\begin{aligned}
& \Psi_{X}=-\log \left(1-A e^{\tau}\right), \\
& \Psi_{Y}=-\log \left(1-B e^{\tau}\right),
\end{aligned}
$$

where $A$ and $B$ are constant along a given ray. Evaluating the eikonal equation in (197) along the corner $X=X_{0}, Y=Y_{0}$ and using (215) with $\tau=0$ gives

$$
1+X_{0}+Y_{0}=\frac{Y_{0}}{1-B}+\frac{X_{0}}{1-A}+1-A
$$

and this leads to the relation between $A$ and $B$ in (67). Here we take $\tau=0$ to correspond to when a ray starts from the corner point $X=X_{0}, Y=Y_{0}$. Using (215) we solve the two ODEs in (199), subject to $\left.X\right|_{\tau=0}=X_{0}$ and $\left.Y\right|_{\tau=0}=Y_{0}$, and we thus obtain the expressions in (68), which give the corner rays in parametric form. Using (215) and (68) we then integrate (200), and choosing $\Psi\left(X_{0}, Y_{0}\right)=0$ for convenience, we obtain (66). Note that $\Psi$ can only be determined from (197) up to an additive constant, but such a constant can be incorporated into $C_{0}=C_{0}\left(\rho ; X_{0}, Y_{0}\right)$ in (195).

We can view $A$ as indexing this family of rays. If $A$ is such that $\left(A+X_{0}+Y_{0}\right)(1-A)-X_{0}=0$ then $B$ in (216) and (67) becomes infinite, and this corresponds to $A=A_{\max }$ in (69). This critical ray corresponds to $Y=Y_{0}$, and for $\tau \in$ $\left(0,-\log \left(A_{\max }\right)\right)$ we have $X \in\left(0, X_{0}\right)$, so the ray is the upper 
boundary of the state space rectangle, where all secondary storage spaces are full. The ray $A=A_{\min }=1-\sqrt{X_{0}}$ (for $X_{0}<$ 1) corresponds to the curve $Y=Y_{c}(X)=Y_{c}\left(X ; X_{0}, Y_{0}\right)$ in (86). For regions $\mathscr{R}_{1} \cup \mathscr{R}_{2} \cup \mathscr{R}_{3}$ we have $Y_{0} /\left(X_{0}+Y_{0}\right)>1-\sqrt{X_{0}}$ and then the ray with $A=Y_{0} /\left(X_{0}+Y_{0}\right)$ is the curve $\tilde{Y}(X)$ in (56). Thus for regions $\mathscr{R}_{1} \cup \mathscr{R}_{2} \cup \mathscr{R}_{3}$ it suffices to consider $A$ in the range $\left(Y_{0} /\left(X_{0}+Y_{0}\right), A_{\text {max }}\right)$ to fill region $\mathscr{D}_{0}$ in state space. We also note that in order for the rays, which all start from $(X, Y)=\left(X_{0}, Y_{0}\right)$, to enter the state space we need $\left.X_{\tau}\right|_{\tau=0}<0$ and $\left.Y_{\tau}\right|_{\tau=0}<0$, which implies that $1-A-X_{0} /(1-A)<0$ and $X_{0} /(1-A)-\left(A+X_{0}+Y_{0}\right)<0$. Adding the last two inequalities implies that $A>(1 / 2)\left(1-X_{0}-Y_{0}\right)$ and this is certainly true if $A>A_{\min }$.

We next solve the transport equation (198) for $K(X, Y)$. This equation can be written as an ODE along a ray, with

$$
\begin{aligned}
\frac{K_{\tau}}{K}= & \left(\frac{1}{2} Y \Psi_{Y Y}+1\right) e^{\Psi_{Y}}+\left(\frac{1}{2} X \Psi_{X X}+1\right) e^{\Psi_{X}} \\
& +\frac{1}{2} \Psi_{X X} e^{-\Psi_{X}} \\
= & e^{\Psi_{X}}+e^{\Psi_{Y}}-\frac{1}{2}\left(X_{\tau \tau}+X_{\tau} e^{\Psi_{X}}\right) \\
& -\frac{1}{2}\left(Y_{\tau \tau}+Y_{\tau} e^{\Psi_{Y}}\right),
\end{aligned}
$$

where we used (199), and the last equality in (217) follows by differentiating (199) with respect to $\tau$. Introducing the Jacobian $\Delta \equiv X_{\tau} Y_{A}-X_{A} Y_{\tau}$ associated with the mapping from $(\tau, A)$ to $(X, Y)$ variables, after some calculation we find that (217) becomes

$$
\frac{K_{\tau}}{K}=\frac{1}{2} \frac{1}{1-A e^{\tau}}+\frac{1}{2} \frac{1}{1-B e^{\tau}}-\frac{1}{2} \frac{\Delta_{\tau}}{\Delta}
$$

and the most general solution to (218) is given by

$$
\begin{aligned}
& K(X, Y) \\
& \quad=\left[\left(e^{-\tau}-A\right)\left(e^{-\tau}-B\right)|\Delta(\tau, A)|\right]^{-1 / 2} K_{0}(A) .
\end{aligned}
$$

Here $K_{0}(A)$ is an arbitrary function of the parameter that indexes the rays and is thus constant along any particular ray. We have thus determined the expansion in (195) up to the constant $C_{0}$ and the function $K_{0}(\cdot)$. To complete the $\mathscr{D}_{0}$ ray expansion we will need to use asymptotic matching to a local expansion valid near the corner $(X, Y)=\left(X_{0}, Y_{0}\right)$. This is constructed in Section 4.2. In order to accomplish the matching we will need the behavior of (195) as $X \uparrow X_{0}$, $Y \uparrow Y_{0}$, and this is examined next.

We note that $(X, Y) \rightarrow\left(X_{0}, Y_{0}\right)$ corresponds to $\tau \rightarrow 0$ and in this limit the Jacobian in (71) vanishes, and

$\Delta$

$$
\begin{array}{r}
\sim(-\tau)\left[\left(1+X_{0}+Y_{0}\right)\left(1+\frac{X_{0}}{(1-A)^{2}}\right)-\frac{4 X_{0}}{1-A}\right], \\
\tau \longrightarrow 0^{+} .
\end{array}
$$

For $\tau \rightarrow 0$ we also have

$$
\begin{aligned}
X_{0}-X & \sim\left(\frac{X_{0}}{1-A}+A-1\right) \tau, \\
Y_{0}-Y & \sim \frac{Y_{0}}{1-B} \tau
\end{aligned}
$$

so that

$$
\begin{aligned}
\frac{Y_{0}-Y}{X_{0}-X}=\frac{p}{n} \sim \frac{\left(A+X_{0}+Y_{0}\right)(1-A)-X_{0}}{X_{0}-(1-A)^{2}} & , \\
\tau \longrightarrow 0 . &
\end{aligned}
$$

Here we recall that $Y=Y_{0}-p / \rho$ and $X=X_{0}-n / \rho$, and (222) gives the slope at which the ray indexed by $A$ hits the corner point $\left(X_{0}, Y_{0}\right)$.

We can thus invert the transformation in (68) locally, with (222) corresponding to a quadratic equation for $A=A(n / p)$, and hence

$$
\begin{aligned}
A & \sim A_{s} \equiv 1+\frac{1}{2} \frac{1}{p-n}\left[\left(1+X_{0}+Y_{0}\right) n\right. \\
& \left.-\sqrt{\left(1+X_{0}+Y_{0}\right)^{2} n^{2}+4\left(p^{2}-n^{2}\right) X_{0}}\right] .
\end{aligned}
$$

Then we define $B_{s}$ by replacing $A$ by $A_{s}$ in (67). Note that the right-hand side of (223) approaches $A_{\max }$ if $n \rightarrow \infty$, approaches $A_{\min }=1-\sqrt{X_{0}}$ as $p \rightarrow \infty$, and is equal to $Y_{0} /\left(X_{0}+Y_{0}\right)$ if $n / p=\left[\left(X_{0}+Y_{0}\right)^{2}-X_{0}\right] / Y_{0}$. For $\tau \rightarrow 0$ we also have $\Psi(X, Y) \rightarrow 0$ with

$$
\begin{aligned}
& \Psi(X, Y)=\left[\left(A+\frac{X_{0}}{1-A}-1\right) \log (1-A)\right. \\
& \left.+\frac{Y_{0}}{1-B} \log (1-B)\right] \tau+O\left(\tau^{2}\right)=\left(X_{0}-X\right) \\
& \quad \cdot \log \left(1-A_{s}\right)+\left(Y_{0}-Y\right) \log \left(1-B_{s}\right)+O\left(\tau^{2}\right)
\end{aligned}
$$

so that $e^{\rho \Psi(X, Y)} \sim\left(1-A_{s}\right)^{n}\left(1-B_{s}\right)^{p}$. We have thus shown that as $\tau \rightarrow 0$ we have

$$
\begin{aligned}
C_{0} K(\mathrm{X}, Y) e^{\rho \Psi(X, Y)} \sim C_{0} \frac{K_{0}\left(A_{s}\right)}{\sqrt{1-A_{s}} \sqrt{1-B_{s}}}(1 \\
\left.-A_{s}\right)^{n}\left(1-B_{s}\right)^{p} \\
\cdot \frac{1}{\sqrt{\tau}}\left[\left(1+X_{0}+Y_{0}\right)\left(1+\frac{X_{0}}{\left(1-A_{s}\right)^{2}}\right)\right. \\
\left.-\frac{4 X_{0}}{1-A_{s}}\right]^{-1 / 2},
\end{aligned}
$$

where $A \sim A_{s}$ was approximated by (223), and by (221) we have

$$
\tau \sim \frac{\left(1-B_{s}\right)\left(Y_{0}-Y\right)}{Y_{0}} \sim \frac{1-B_{s}}{Y_{0}} \frac{p}{\rho},
$$

so that (225) becomes an explicit function of $n$ and $p$. 
4.2. Analysis of the Scale $k=m-O(1), r=R-O(1)$. We use the variables $n=m-k$ and $p=R-r$ and obtain an approximation to $\pi(k, r)$ that is valid when all but a few of the primary and secondary spaces are occupied. This is likely for parameter regions $\mathscr{R}_{3}$ and $\mathscr{R}_{4}$ but unlikely for $\mathscr{R}_{1}$ and $\mathscr{R}_{2}$. We define $Q$ by

$$
\pi(k, r)=\pi(k, r ; \rho, m, R)=Q\left(n, p ; \rho, X_{0}, Y_{0}\right) .
$$

We thus denote by $Q(n, p)$ the exact probability that there are $n$ (resp., $p$ ) empty primary (resp., secondary) spaces, and we will later denote by $Q_{L}(n, p)$ the leading term in an asymptotic expansion of this probability, which is valid for the scale $n, p=O(1)$. Then clearly $Q(0,0) \sim Q_{L}(0,0)$ so in asymptotic relations involving $Q(0,0)$ we can drop the subscript $L$.

Writing the balance equations in (3), (4), and (5) in terms of $(n, p)$ leads to

$$
\begin{aligned}
{[1+} & \left.X_{0}+Y_{0}-\rho^{-1}(n+p)\right] Q(n, p) \\
= & {\left[Y_{0}+\rho^{-1}(1-p)\right] Q(n, p-1) } \\
& +\left[X_{0}+\rho^{-1}(1-n)\right] Q(n-1, p) \\
& +Q(n+1, p) ; \quad n \geqslant 1, p \geqslant 1, \\
{[1+} & \left.X_{0}+Y_{0}-\rho^{-1} p\right] Q(0, p) \\
= & {\left[Y_{0}+\rho^{-1}(1-p)\right] Q(0, p-1)+Q(1, p) } \\
& +Q(0, p+1), \quad p \geqslant 1, \\
{[1+} & \left.X_{0}+Y_{0}-\rho^{-1} n\right] Q(n, 0) \\
= & {\left[X_{0}+\rho^{-1}(1-n)\right] Q(n-1,0)+Q(n+1,0), }
\end{aligned}
$$

$n \geqslant 1$

and the corner condition in (9) becomes

$$
\left(X_{0}+Y_{0}\right) Q(0,0)=Q(1,0)+Q(0,1) .
$$

Note that near this corner only the boundaries $X=X_{0}$ and $Y=Y_{0}$ of the state space rectangle are relevant, and the problem in (228)-(231) corresponds to a random walk in a quarter plane, in $(n, p)$ space. For $\rho \rightarrow \infty$ the problem in (228)-(231) may be further approximated by

$$
\begin{aligned}
(1+ & \left.X_{0}+Y_{0}\right) Q_{L}(n, p) \\
= & Y_{0} Q_{L}(n, p-1)+X_{0} Q_{L}(n-1, p) \\
& \quad+Q_{L}(n+1, p) ; n \geqslant 1, p \geqslant 1, \quad p \geqslant 1, \\
(1+ & \left.X_{0}+Y_{0}\right) Q_{L}(0, p) \\
= & Y_{0} Q_{L}(0, p-1)+Q_{L}(1, p)+Q_{L}(0, p+1), \\
(1+ & \left.X_{0}+Y_{0}\right) Q_{L}(n, 0) \\
= & X_{0} Q_{L}(n-1,0)+Q_{L}(n+1,0), \quad n \geqslant 1 .
\end{aligned}
$$

Here $Q_{L}(n, p)$ in (232)-(234) is understood to be the leading term in an asymptotic expansion of $Q(n, p ; \rho)$, for $\rho \rightarrow \infty$, and the corner equation in (231) must also be satisfied by this leading term.

We introduce the double generating function

$$
\widetilde{Q}(z, w)=\sum_{n=0}^{\infty} \sum_{p=0}^{\infty} Q_{L}(n, p) z^{n} w^{p}
$$

and from (231)-(234) we obtain

$$
\begin{aligned}
& {\left[1+X_{0}(1-z)+Y_{0}(1-w)-\frac{1}{z}\right] \widetilde{Q}(z, w)} \\
& =\left(\frac{1}{w}-\frac{1}{z}\right) \widetilde{Q}(0, w)+\left(1-\frac{1}{w}\right) Q_{L}(0,0) .
\end{aligned}
$$

From (236), by setting $w=1$ we obtain

$$
\widetilde{Q}(z, 1)=\frac{\widetilde{Q}(0,1)}{1-z X_{0}}
$$

and if $z=w$ we find that $\widetilde{Q}(z, z)=Q_{L}(0,0) /\left[1-\left(X_{0}+Y_{0}\right) z\right]$. Setting $z=w$ then in (235) leads to

$$
\sum_{n+p=\ell} Q_{L}(n, p)=Q_{L}(0,0)\left(X_{0}+Y_{0}\right)^{\ell}, \quad \ell \geqslant 0
$$

The expression in (238) shows that the total number of empty spaces $(=m+R-k-r=n+p)$ follows asymptotically a geometric distribution if $X_{0}+Y_{0}<1$, and this could be also deduced from (12), by expanding the exact (truncated Poisson) distribution for $\rho \rightarrow \infty$. Note, however, that (238) holds also for $X_{0}+Y_{0} \geqslant 1$. The factor in brackets in the lefthand side of (236) vanishes when $z=z_{ \pm}(w)$, where $z_{ \pm}$are given in (51). Requiring $\widetilde{Q}(z, w)$ to be analytic at the smaller root $z_{-}(w)$ determines $\widetilde{Q}(0, w)$ as

$$
\widetilde{Q}(0, w)=\frac{z_{+}(w)(1-w)}{\left(z_{-}(w)-w\right) X_{0}} Q_{L}(0,0) .
$$

Then using (239) in (236) and partially inverting the transform in (236) lead to

$$
\begin{aligned}
& \sum_{p=0}^{\infty} w^{p} Q_{L}(n, p) \\
& \quad=\frac{1-w}{\left(z_{-}(w)-w\right) X_{0}}\left[z_{+}(w)\right]^{-n-1} Q_{L}(0,0),
\end{aligned}
$$

$n \geqslant 0$,

and hence by the Cauchy integral formula

$$
\begin{aligned}
Q_{L} & (n, p) \\
& =\frac{Q_{L}(0,0)}{X_{0}(2 \pi i)} \oint \frac{1-w}{z_{-}(w)-w}\left[z_{+}(w)\right]^{-n-1} w^{-p-1} d w,
\end{aligned}
$$

where the contour is over a small loop about $w=0$. 
When $X_{0}+Y_{0}<1\left(m+R<\rho\right.$, region $\left.\mathscr{R}_{3} \cup \mathscr{R}_{4}\right)$, most primary and secondary spaces will be full, and then (241) becomes a normalized discrete distribution with

$$
Q(0,0) \sim Q_{L}(0,0)=1-X_{0}-Y_{0}, \quad \mathscr{R}_{3} \cup \mathscr{R}_{4},
$$

leading to the limit law in (50).

For regions $\mathscr{R}_{1} \cup \mathscr{R}_{2}$, (241) still represents a local approximation to $\pi(k, r)$, but now different arguments must be used to determine $Q_{L}(0,0)$, and indeed now $Q_{L}(0,0)$ will turn out to be exponentially small for large $\rho$. We proceed to relate (241) to the ray expansions in $\mathscr{D}_{0}, \mathscr{D}_{+}$, and $\mathscr{D}_{-}$, noting that, for parameter region $\mathscr{R}_{1} \cup \mathscr{R}_{2} \cup \mathscr{R}_{3}, \mathscr{D}_{0}$ and $\mathscr{D}_{+}$both border the corner point, while, for $\mathscr{R}_{4}, \mathscr{D}_{0}$ and $\mathscr{D}_{\text {- border }}$ this point. We will need to expand (241) asymptotically, for $n$ and/or $p \rightarrow \infty$. The integrand in (241) has branch points at $w=\left(1+X_{0}+Y_{0} \pm 2 \sqrt{X_{0}}\right) / Y_{0}$ and possible poles at solutions of $z_{-}(w)=w$. Now, $z_{-}(1)=1$ for $X_{0}>1$ but $w=1$ is not a pole in view of the factor $1-w$ in the numerator. The only other possible solution to $z_{-}(w)=w$ occurs at $w=1 /\left(X_{0}+Y_{0}\right)$ and this is a pole if

$$
\begin{aligned}
\frac{2 X_{0}}{X_{0}+Y_{0}}= & 1+X_{0}+Y_{0}-\frac{Y_{0}}{X_{0}+Y_{0}} \\
& -\sqrt{\left(1+X_{0}+Y_{0}-\frac{Y_{0}}{X_{0}+Y_{0}}\right)^{2}-4 X_{0}} \\
= & X_{0}+Y_{0}+\frac{X_{0}}{X_{0}+Y_{0}}-\frac{\left|\left(X_{0}+Y_{0}\right)^{2}-X_{0}\right|}{X_{0}+Y_{0}} .
\end{aligned}
$$

But (243) holds precisely when $\left(X_{0}+Y_{0}\right)^{2}>X_{0}$, which is true for regions $\mathscr{R}_{1} \cup \mathscr{R}_{2} \cup \mathscr{R}_{3}$. Thus for $\mathscr{R}_{4}$ the pole is absent and along the transition curve $\mathscr{R}_{3} \cap \mathscr{R}_{4}$ (cf. (25)) the pole coalesces with the lower branch point, both being at $w=1 / \sqrt{X_{0}}(>1)$. We can recast the integral in (241) by using the conformal map $u=z_{+}(w)$, so that the inverse is

$$
w=1+\frac{1}{Y_{0}}\left(1+X_{0}-X_{0} u-\frac{1}{u}\right)
$$

and (241) becomes

$$
\begin{aligned}
& Q_{L}(n, p)=\frac{Q_{L}(0,0)}{2 \pi i} \\
& \quad \cdot \int_{\Gamma} \frac{(1-u)\left(X_{0} u^{2}-1\right) Y_{0}^{p} u^{p-n-2}}{\left(X_{0}+Y_{0}-X_{0} u\right)\left[\left(1+X_{0}+Y_{0}\right) u-X_{0} u^{2}-1\right]^{p+1}} d u,
\end{aligned}
$$

where $\Gamma$ is a small loop about $u=z_{+}(0)$, with

$z_{+}(0)$

$$
=\frac{1}{2 X_{0}}\left[1+X_{0}+Y_{0}+\sqrt{\left(1+X_{0}+Y_{0}\right)^{2}-4 X_{0}}\right],
$$

and the integrand in (245) has a pole of order $p+1$ at $u=$ $z_{+}(0)$. Below we collect some asymptotic results for $Q_{L}(n, p)$ that will be used in the matching calculations.

Proposition 20. For $n$ and/or $p \rightarrow \infty$ the function $Q_{L}(n, p)$ has the following asymptotic expansions: (i) $p=O(1), n \rightarrow \infty$

$$
\begin{aligned}
Q_{L}(n, p) \sim & Q(0,0) \frac{\left[z_{+}(0)\right]^{-n}}{p !} \\
& \cdot n^{p}\left(\frac{Y_{0}}{\sqrt{\left(1+X_{0}+Y_{0}\right)^{2}-4 X_{0}}}\right)^{p} .
\end{aligned}
$$

(ii) $p, n \rightarrow \infty$ with $0 \leqslant n / p<\theta_{1}, \theta_{1}=\left[\left(X_{0}+Y_{0}\right)^{2}-\right.$ $\left.X_{0}\right] / Y_{0}$, for $\left(X_{0}, Y_{0}\right) \in \mathscr{R}_{1} \cup \mathscr{R}_{2} \cup \mathscr{R}_{3}$

$Q_{L}(n, p)$

$\sim Q(0,0)\left[\left(X_{0}+Y_{0}\right)^{2}-X_{0}\right] X_{0}^{n}\left(X_{0}+Y_{0}\right)^{p-n-2}$.

(iii) $p, n \rightarrow \infty$ with $\theta_{1}<n / p<\infty$, for $\mathscr{R}_{1} \cup \mathscr{R}_{2} \cup \mathscr{R}_{3}$

$$
\begin{aligned}
& Q_{L}(n, p) \\
& \sim \frac{Q(0,0)}{\sqrt{2 \pi}}\left[n^{2}\left(1+X_{0}+Y_{0}\right)^{2}+4\left(p^{2}-n^{2}\right) X_{0}\right]^{-1 / 4} \\
& \cdot \frac{\left(1-u_{s}\right)\left(X_{0} u_{s}^{2}-1\right)}{u_{s}^{3 / 2}\left(X_{0}+Y_{0}-X_{0} u_{s}\right) \sqrt{\left(1+X_{0}+Y_{0}\right) u_{s}-1-X_{0} u_{s}^{2}}} \\
& \cdot u_{s}^{p-n} Y_{0}^{p}\left[\left(1+X_{0}+Y_{0}\right) u_{s}-1-X_{0} u_{s}^{2}\right]^{-p} \text {, }
\end{aligned}
$$

where

$$
\begin{aligned}
u_{s} & =u_{s}\left(\frac{n}{p}\right)=\frac{1}{2 X_{0}(n+p)}\left[\left(1+X_{0}+Y_{0}\right) n\right. \\
& \left.+\sqrt{\left(1+X_{0}+Y_{0}\right)^{2} n^{2}+4 X_{0}\left(p^{2}-n^{2}\right)}\right] .
\end{aligned}
$$

(iv) $p, n \rightarrow \infty, n / p \approx \theta_{1}$ (with $n-p \theta_{1}=O(\sqrt{p})$ ), for $\mathscr{R}_{1} \cup \mathscr{R}_{2} \cup \mathscr{R}_{3}$

$$
\begin{aligned}
& Q_{L}(n, p) \sim Q(0,0) X_{0}^{n}\left(X_{0}+Y_{0}\right)^{p-n-2}\left[\left(X_{0}+Y_{0}\right)^{2}-X_{0}\right] \frac{1}{\sqrt{2 \pi}} \\
& \cdot \int_{-\infty}^{\eta} e^{-v^{2} / 2} d v
\end{aligned}
$$

$$
\begin{aligned}
\eta & =\frac{Y_{0} \sqrt{p}}{\sqrt{X_{0}+Y_{0}} \sqrt{\left(X_{0}+Y_{0}\right)^{3}+X_{0}+\left(X_{0}+Y_{0}\right)\left(Y_{0}-2 X_{0}\right)}}\left(\theta_{1}\right. \\
& \left.-\frac{n}{p}\right)=O(1) .
\end{aligned}
$$

(v) $p, n \rightarrow \infty, 0<n / p \leqslant \infty$, for region $\mathscr{R}_{4}$.

The expression in (249) holds for all $0<n / p<\infty$ and (247) holds for $n \rightarrow \infty$ with $p=O(1)$. 
(vi) $n=O(1), p \rightarrow \infty$, for region $\mathscr{R}_{4}$

$$
\begin{aligned}
& Q_{L}(n, p) \sim \frac{Q(0,0)}{2 \sqrt{\pi}} X_{0}^{n / 2-1 / 4}\left(\frac{Y_{0}}{Y_{0}+W_{0}}\right)^{p} \\
& \frac{\sqrt{W_{0}+Y_{0}}\left(1-\sqrt{X_{0}}\right)}{\sqrt{X_{0}}-X_{0}-Y_{0}} \\
& \cdot p^{-3 / 2}\left[n+\frac{\sqrt{X_{0}}}{\sqrt{X_{0}}-1}+\frac{\sqrt{X_{0}}}{\sqrt{X_{0}}-X_{0}-Y_{0}}\right] .
\end{aligned}
$$

(vii) $n, p \rightarrow \infty$ with $n=O(\sqrt{p})$, for region $\mathscr{R}_{3} \cap \mathscr{R}_{4}$, with $Y=\sqrt{X_{0}}-X_{0}+\rho^{-1 / 2} \delta, \delta=O(1)$

$$
\begin{aligned}
& Q_{L}(n, p) \sim \frac{Q(0,0)}{\sqrt{\pi}} X_{0}^{n / 2}\left(\frac{Y_{0}}{Y_{0}+W_{0}}\right)^{p}\left\{\frac{\sqrt{1-\sqrt{X_{0}}}}{X_{0}^{1 / 4} \sqrt{p}}\right. \\
& \cdot \exp \left[\frac{n^{2}}{4 p}\left(1-\frac{1}{\sqrt{X_{0}}}\right)\right]+\frac{\delta}{\sqrt{\rho}} \sqrt{\frac{2}{X_{0}}} \\
& \cdot \exp \left[\frac{p \delta^{2}}{\rho \sqrt{X_{0}}\left(1-\sqrt{X_{0}}\right)}-\frac{n \delta}{\sqrt{\rho} \sqrt{X_{0}}}\right] \\
& \left.\cdot\left(\int_{\eta_{0}}^{\infty} e^{-v^{2} / 2} d v\right)\right\}, \\
& \eta_{0}=\frac{\sqrt{1-\sqrt{X_{0}}}}{\sqrt{2} X_{0}^{1 / 4}} \frac{\sqrt{2}}{\sqrt{p}}-\frac{\sqrt{1-\sqrt{X_{0}}} X_{0}^{1 / 4}}{\frac{\sqrt{p} \delta}{\sqrt{\rho}} .}
\end{aligned}
$$

The results in (247)-(255) may be obtained by expanding the integrals in (241) or (245) by a combination of singularity analysis and the saddle point method. Good references on asymptotic expansion of integrals are the books [15-19], but since these methods are now well established, we merely sketch the proof of Proposition 20.

To obtain (247) we approximate the integrand in (241) for $w=O\left(n^{-1}\right)$, scaling $w=w_{1} / n$ and using

$$
\begin{aligned}
& {\left[z_{+}(w)\right]^{-n-1}} \\
& \quad \sim\left[z_{+}(0)\right]^{-n-1}\left[1+\frac{w z_{+}^{\prime}(0)}{z_{+}(0)}+O\left(w^{2}\right)\right]^{-n-1} \\
& \quad \sim\left[z_{+}(0)\right]^{-n-1} \exp \left(-\frac{z_{+}^{\prime}(0)}{z_{+}(0)} w_{1}\right)
\end{aligned}
$$

Note that (247) is not only asymptotically true but also an exact expression when $p=0$, since $Q(n, 0)$ satisfies the boundary equation in (234). Turning to (245) we see that the integrand has a simple pole at $u=u_{*} \equiv\left(X_{0}+Y_{0}\right) / X_{0}$ and saddle point(s) where

$$
\begin{aligned}
& \frac{d}{d u}\{(p-n) \log u \\
& \left.\quad-p \log \left[\left(1+X_{0}+Y_{0}\right) u-X_{0} u^{2}-1\right]\right\}=0,
\end{aligned}
$$

and (257) is equivalent to the quadratic equation

$$
\left(\frac{n}{p}+1\right) X_{0} u^{2}-\frac{n}{p}\left(1+X_{0}+Y_{0}\right) u+\frac{n}{p}-1=0 .
$$

One root of (258) is given by $u_{s}=u_{s}(n / p)$ in (250), and the complementary root, with a minus sign in front of the square root, will correspond to a second saddle which will not play any role in the analysis. The pole $u_{*}$ and saddle $u_{s}$, whose location depends on the ratio $n / p$, coalesce when $n / p=\theta_{1}$, for regions $\mathscr{R}_{1}, \mathscr{R}_{2}$, and $\mathscr{R}_{3}$. Note also that as $n / p \rightarrow \infty$ we have $u_{s} \rightarrow z_{+}(0)$ and that the integrand in (245) has a zero at $u=1 / \sqrt{X_{0}}$. For region $\mathscr{R}_{1} \cup \mathscr{R}_{2} \cup \mathscr{R}_{3}$ and $n / p \in\left(0, \theta_{1}\right)$ we have the ordering

$$
\frac{1}{\sqrt{X_{0}}}<u_{s}<u_{*}<z_{+}(0)
$$

and we note that $u_{*}<z_{+}(0)$ is always true. Then we dilate the small loop $\Gamma$ about $u=z_{+}(0)$ to the saddle point contour $\left|u-z_{+}(0)\right|=\left|u_{s}-z_{+}(0)\right|$, which is a circular contour that traverses the saddle $u_{s}$ in the steepest descent directions, which are $\arg \left(u-u_{s}\right)= \pm \pi / 2$. But in doing the dilation we must take into account the contribution from the residue at the pole $u=u_{*}$, in view of (259). It turns out that the residue dominates the saddle point contribution, and we thus obtain the expression in (248). For $n / p \in\left(\theta_{1}, \infty\right)$ we have the ordering

$$
\frac{1}{\sqrt{X_{0}}}<u_{*}<u_{s}<z_{+}(0)
$$

and then the pole does not contribute as we dilate $\Gamma$ to the saddle point contour. Setting

$$
\begin{gathered}
G(u)=\frac{(1-u)\left(X_{0} u^{2}-1\right)}{\left(X_{0}+Y_{0}\right) u-1} \\
\cdot \frac{1}{u^{2}\left[\left(1+X_{0}+Y_{0}\right) u-X_{0} u^{2}-1\right]}, \\
F(u)=F\left(u ; \frac{n}{p}\right)=\log Y_{0}+\left(1-\frac{n}{p}\right) \log u \\
-\log \left[\left(1+X_{0}+Y_{0}\right) u-X_{0} u^{2}-1\right]
\end{gathered}
$$

we use for (245) the standard saddle point estimate

$$
\begin{aligned}
& \frac{1}{2 \pi i} \int_{\Gamma} G(u) e^{p F(u)} d u=\frac{1}{2 \pi i} \int_{\Gamma_{s}} G(u) e^{p F(u)} d u \\
& \sim \frac{1}{\sqrt{2 \pi p}} \frac{G\left(u_{s}\right)}{\sqrt{F^{\prime \prime}\left(u_{s}\right)}} e^{p F\left(u_{s}\right)},
\end{aligned}
$$

and this leads to the expression in (249). The transitional result in (251) corresponds to $n / p \approx \theta_{1}$ and then we have $u_{*} \approx$ $u_{s}$, so the saddle is close to a simple pole. Such situations are discussed in detail in [15], and by expanding the integrand in (245) about $u_{*}$ we ultimately obtain a simpler integrand that is 
related to $D_{-1}(\cdot)$, the parabolic cylinder function of order -1 , which can in turn be expressed in terms of the standard error function, leading to (251). Note that as $\eta \rightarrow+\infty$ we approach the region $n / p<\theta_{1}$ and then (251) reduces to (248).

The pole at $u_{*}$ and zero at $1 / \sqrt{X_{0}}$ coalesce when $X_{0}+Y_{0}=$ $\sqrt{X_{0}}$, which is precisely the curve $\mathscr{R}_{3} \cap \mathscr{R}_{4}$ which separates $\mathscr{R}_{3}$ from $\mathscr{R}_{4}$ in parameter space. For $\left(X_{0}, Y_{0}\right) \in \mathscr{R}_{4}$ we have the ordering

$$
u_{*}<\frac{1}{\sqrt{X_{0}}}<u_{s}<z_{+}(0) .
$$

Then for any $n / p \in(0, \infty)$ we can deform $\Gamma$ into the saddle point contour and obtain (249) as the approximation to $Q_{L}(n, p)$. The limits $n=O(1), p \rightarrow \infty$, and $n \rightarrow \infty$, $p=O(1)$ require a separate analysis. For the latter $u_{s}$ becomes close to $z_{+}(0)$ and we again obtain (247), while for the former the saddle $u_{s}$ gets close to zero at $1 / \sqrt{X_{0}}$. By expanding the integrand near $u=1 / \sqrt{X_{0}}$, setting $u=1 / \sqrt{X_{0}}+v / \sqrt{p}$, we obtain

$$
\begin{gathered}
Q_{L}(n, p)=Q_{L}(0,0)\left(\sqrt{X_{0}}\right)^{n}\left(\frac{Y_{0}}{Y_{0}+W_{0}}\right)^{p} \frac{X_{0}}{Y_{0}+W_{0}} \\
\cdot \frac{1}{X_{0}+Y_{0}-\sqrt{X_{0}}} \frac{1}{2 \pi i} \int_{-i \infty}^{i \infty} \frac{v}{p}\left[2 \sqrt{X_{0}}\left(\sqrt{X_{0}}-1\right)\right. \\
\left.+\mathscr{B} \frac{v}{\sqrt{p}}+O\left(p^{-1}\right)\right]\left[1-\sqrt{X_{0}} n \frac{v}{\sqrt{p}}\right][1 \\
\left.-\frac{X_{0}^{2}}{Y_{0}+W_{0}} \frac{v^{3}}{\sqrt{p}}\right] \exp \left(\frac{X_{0}^{3 / 2} v^{2}}{Y_{0}+W_{0}}\right) d v,
\end{gathered}
$$

where

$$
\mathscr{B}=-5 X_{0}^{3 / 2}+3 X_{0}+\frac{2 X_{0}^{3 / 2}\left(\sqrt{X_{0}}-1\right)}{X_{0}+Y_{0}-\sqrt{X_{0}}} .
$$

Evaluating the integral(s) in (264) we obtain (253). Note that the coefficient of the $O\left(p^{-1}\right)$ term in the integral is zero, so the result is $O\left(p^{-3 / 2}\right)$.

When $Y_{0}+X_{0} \approx \sqrt{X_{0}}$ the pole at $u_{*}$ and zero at $1 / \sqrt{X_{0}}$ are close together. If $n, p \rightarrow \infty$ so that $n / p$ remains fixed and positive, the saddle lies well to the right of these, and then (249) holds. But if $n, p \rightarrow \infty$ with $n=O(\sqrt{p})$ then the saddle, pole, and zero are all close. Then we must reexamine the integrand in (245) and expand it about $u=1 / \sqrt{X_{0}}$. Again setting $v=\sqrt{\rho}\left(u-1 / \sqrt{X_{0}}\right)=O(1)$ and replacing $Y_{0}$ by $\sqrt{X_{0}}-X_{0}+\delta / \sqrt{\rho}$ (with $\delta=O(1)$ ), (245) becomes asymptotically

$$
\begin{aligned}
& Q_{L}(n, p) \sim Q(0,0) X_{0}^{n / 2}\left(\frac{Y_{0}}{Y_{0}+W_{0}}\right)^{p} \frac{2 \sqrt{X_{0}}}{\sqrt{\rho}} \frac{1}{2 \pi i} \\
& \cdot \int_{\mathrm{Br}_{+}} \frac{X_{0} v}{X_{0} v-\delta} \exp \left[-\frac{n}{\sqrt{\rho}} \sqrt{X_{0}} v\right. \\
& \left.+\frac{p}{\rho} \frac{X_{0}^{3 / 2}}{Y_{0}+W_{0}} v^{2}\right] d v,
\end{aligned}
$$

where $\mathrm{Br}_{+}$is a vertical contour in the complex $v$-plane, which is to the right of the pole at $v=\delta / X_{0}$. The integral in (266) may be evaluated as a combination of a Gaussian and an error function, and this ultimately leads to (254) with (255). Since the large parameter $\rho$ appears explicitly in (254) and (266) we can define the asymptotic limit more precisely, as $\rho \rightarrow \infty$ with $Y_{0}+X_{0}-\sqrt{X_{0}}=O\left(\rho^{-1 / 2}\right), p=O(\rho)$, and $n=O(\sqrt{\rho})$.

With Proposition 20 we are now ready to relate the corner approximation $Q_{L}(n, p)$ to the various ray expansions, via asymptotic matching. First consider parameter region $\mathscr{R}_{1} \cup$ $\mathscr{R}_{2} \cup \mathscr{R}_{3}$, so that $\mathscr{D}_{0}$ and $\mathscr{D}_{+}$come together at the corner point $\left(X_{0}, Y_{0}\right)$. In $\mathscr{D}_{+}$the function $\Psi_{+}(X, Y)$ can be expanded in Taylor series about $(X, Y)=\left(X_{0}, Y_{0}\right)$ and as $(X, Y) \rightarrow$ $\left(X_{0}, Y_{0}\right)$ we obtain

$$
\begin{aligned}
& C(\rho) K_{+}(X, Y) e^{\rho \Psi_{+}(X, Y)} \sim C(\rho) K_{+}\left(X_{0}, Y_{0}\right) \\
& \cdot e^{\rho \Psi_{+}\left(X_{0}, Y_{0}\right)} \exp \left[\rho\left(X-X_{0}\right) \Psi_{+, X}\left(X_{0}, Y_{0}\right)\right. \\
& \left.+\rho\left(Y-Y_{0}\right) \Psi_{+, Y}\left(X_{0}, Y_{0}\right)\right]=C(\rho) \\
& \quad \cdot \frac{\left(X_{0}+Y_{0}\right)^{2}-X_{0}}{\left(X_{0}+Y_{0}\right)^{5 / 2} \sqrt{2 \pi}} \\
& \quad \cdot e^{\rho\left[X_{0}+Y_{0}-1-\left(X_{0}+Y_{0}\right) \log \left(X_{0}+Y_{0}\right)\right]}\left(\frac{X_{0}}{X_{0}+Y_{0}}\right)^{n}\left(X_{0}\right. \\
& \left.+Y_{0}\right)^{p}
\end{aligned}
$$

where we used $\rho\left(X-X_{0}\right)=-n$ and $\rho\left(Y-Y_{0}\right)=-p$. Near $X=X_{0}$, the curve $Y=\tilde{Y}(X)$ that separates $\mathscr{D}_{0}$ from $\mathscr{D}_{+}$has the slope $\tilde{Y}^{\prime}\left(X_{0}\right)=Y_{0} /\left[\left(X_{0}+Y_{0}\right)^{2}-X_{0}\right]$ so that $\tilde{Y}(X)$ can be approximated by the straight line $Y-Y_{0}=\tilde{Y}^{\prime}\left(X_{0}\right)\left(X-X_{0}\right)$, which is the same as $p / n=1 / \theta_{1}$. Then $\mathscr{D}_{+}$meets the corner in the sector $n / p \in\left[0, \theta_{1}\right)$, and this corresponds precisely to where the asymptotic result (248) applies. Comparing (267) to $(248)$ we see that the matching is possible, if $Q(0,0)$ and $C(\rho)$ are related by

$$
\begin{aligned}
& C(\rho) \\
& \sim \sqrt{2 \pi} \sqrt{X_{0}+Y_{0}} e^{\rho\left[1-X_{0}-Y_{0}+\left(X_{0}+Y_{0}\right) \log \left(X_{0}+Y_{0}\right)\right]} Q(0,0)
\end{aligned}
$$

and this holds throughout $\mathscr{R}_{1} \cup \mathscr{R}_{2} \cup \mathscr{R}_{3}$. For $\mathscr{R}_{1} \cup \mathscr{R}_{2}$ we have previously determined that $C(\rho) \sim \rho^{-1 / 2}$ and thus (268) determines $Q(0,0)$, as in (30). For region $\mathscr{R}_{3}$ we have $Q(0,0) \sim 1-X_{0}-Y_{0}$ and then (268) leads to (33). For $\mathscr{R}_{1} \cap \mathscr{R}_{2}$, corresponding to $X_{0}+Y_{0}-1=\gamma / \sqrt{\rho}=O\left(\rho^{-1 / 2}\right)$, the approximation in (208) holds for $n=O(1)$ and $Y-\left(1-X_{0}\right)=$ $O\left(\rho^{-1 / 2}\right)$, but now the upper boundary $Y=Y_{0}$ lies within this range. Then applying the normalization condition in (10) to (208) leads to

1

$$
\sim \sum_{p=0}^{\infty} \sum_{n=0}^{\infty} \frac{C}{\sqrt{2 \pi}}\left(1-X_{0}\right) X_{0}^{n} \exp \left[-\frac{\rho}{2}\left(Y+X_{0}-1\right)^{2}\right]
$$




$$
\begin{aligned}
& =\frac{C}{\sqrt{2 \pi}} \sum_{p=0}^{\infty} \exp \left[-\frac{\rho}{2}\left(\frac{\gamma}{\sqrt{\rho}}-\frac{p}{\rho}\right)^{2}\right] \\
& \sim \frac{\sqrt{\rho}}{\sqrt{2 \pi}} C \int_{0}^{\infty} e^{-(\gamma-v)^{2} / 2} d v=\frac{\sqrt{\rho}}{\sqrt{2 \pi}} C \int_{-\infty}^{\gamma} e^{-v^{2} / 2} d v
\end{aligned}
$$

and then $C$ is given by (33). Then $Q_{L}(0,0) \sim Q(0,0)$ can be computed from (268), and since $\rho\left[1-X_{0}-Y_{0}+\left(X_{0}+\right.\right.$ $\left.\left.Y_{0}\right) \log \left(X_{0}+Y_{0}\right)\right] \sim \gamma^{2} / 2$, we obtain the expression in (29). We have thus determined both $Q(0,0)$ and $C(\rho)$ for all cases of the parameters $X_{0}, Y_{0}$.

To match $Q(n, p)$ to the ray expansion in $\mathscr{D}_{0}$ we note that, for regions $\mathscr{R}_{1} \cup \mathscr{R}_{2} \cup \mathscr{R}_{3}, \mathscr{D}_{0}$ meets the corner over the sector $n / p \in\left(\theta_{1}, \infty\right)$, while for region $\mathscr{R}_{4}$ this holds for all $n / p \in(0, \infty)$. We recall that for $\mathscr{R}_{4}$ the curve $Y=Y_{c}(X)$ separates $\mathscr{D}_{0}$ from $\mathscr{D}_{-}$, but this curve has infinite slope at $X=X_{0}$ and is thus tangent to the line $X=X_{0}$ at $Y=Y_{0}$. So if we approach the corner along any straight line (excluding slopes 0 and $\infty$ ) we are approaching from within $\mathscr{D}_{0}$. By the asymptotic matching principle, the expansion of $Q_{L}(n, p)$ for $n, p \rightarrow \infty$ should agree with the expansion of $C_{0} K e^{\rho \Psi}$ as $X \rightarrow X_{0}, Y \rightarrow Y_{0}$. Thus in $\mathscr{D}_{0}$ we must compare (249) to (225). But from (250) and (223) we see that $\left(1-A_{s}\right) u_{s}=1$ and from (67) we obtain

$$
1-B_{s}=\frac{Y_{0} u_{s}}{\left(X_{0}+Y_{0}-1\right) u_{s}-1-X_{0} u_{s}^{2}} .
$$

It follows that

$$
\begin{aligned}
& \left(1-A_{s}\right)^{n}\left(1-B_{s}\right)^{p} \\
& \quad=u_{s}^{-n}\left\{\frac{u_{s} Y_{0}}{\left[\left(1+X_{0}+Y_{0}\right) u_{s}-1-X_{0} u_{s}^{2}\right]}\right\}^{p}
\end{aligned}
$$

and the matching is possible if

$$
\begin{gathered}
\frac{C_{0} K_{0}\left(A_{s}\right) \sqrt{Y_{0}}}{\sqrt{1-A_{s}}\left(1-B_{s}\right)} \sqrt{\frac{\rho}{p}}\left[\left(1+X_{0}+Y_{0}\right)\left(1+\frac{X_{0}}{\left(1-A_{s}\right)^{2}}\right)-\frac{4 X_{0}}{1-A_{s}}\right]^{-1 / 2} \\
\sim \frac{Q(0,0)}{\sqrt{2 \pi}} \frac{\left(1-u_{s}\right)\left(X_{0} u_{s}^{2}-1\right)\left[n^{2}\left(1+X_{0}+Y_{0}\right)^{2}+4\left(p^{2}-n^{2}\right) X_{0}\right]^{-1 / 4}}{u_{s}^{3 / 2}\left(X_{0}+Y_{0}-X_{0} u_{s}\right) \sqrt{\left(1+X_{0}+Y_{0}\right) u_{s}-1-X_{0} u_{s}^{2}}} .
\end{gathered}
$$

Using (258) to express $n / p$ in terms of $u_{s}$, after some calculation we find that

$$
\begin{aligned}
(1 & \left.+X_{0}+Y_{0}\right)^{2} \frac{n^{2}}{p^{2}}+4 X_{0}\left(1-\frac{n^{2}}{p^{2}}\right) \\
& =\left[\frac{\left(X_{0} u_{s}^{2}+1\right)\left(1+X_{0}+Y_{0}\right)-4 X_{0} u_{s}}{X_{0} u_{s}^{2}+1-\left(1+X_{0}+Y_{0}\right) u_{s}}\right]^{2} .
\end{aligned}
$$

Then choosing $C_{0}(\rho)=Q_{L}(0,0) / \sqrt{\rho}$ we have

$$
C_{0}(\rho) \sim \frac{1}{\sqrt{\rho}} Q(0,0) \quad\left(\mathscr{R}_{1} \cup \mathscr{R}_{2} \cup \mathscr{R}_{3} \cup \mathscr{R}_{4}\right)
$$

Then we use (273) to simplify (272) to

$$
\begin{aligned}
& \frac{K_{0}\left(A_{s}\right) \sqrt{Y_{0}}}{\sqrt{1-A_{s}}\left(1-B_{s}\right)}=\frac{1}{\sqrt{2 \pi}} \frac{1-u_{s}}{u_{s}^{3 / 2}} \frac{X_{0} u_{s}^{2}-1}{X_{0}+Y_{0}-X_{0} u_{s}} \\
& =\frac{1}{\sqrt{2 \pi}} \frac{A_{s}\left[\left(1-A_{s}\right)^{2}-X_{0}\right]}{\sqrt{1-A_{s}}\left[Y_{0}-\left(X_{0}+Y_{0}\right) A_{s}\right]}
\end{aligned}
$$

so we have determined the functional form of $K_{0}(\cdot)$, and (275) along with (219) leads to (70). We have thus completely determined the ray expansion in $\mathscr{D}_{0}$, as $C_{0}$ is known for all parameter regions $\mathscr{R}_{j}$ via (274).
It remains only to determine the constant $C_{1}$ in the caustic ray expansion, for regions $\mathscr{R}_{4}$ and $\mathscr{R}_{3} \cap \mathscr{R}_{4}\left(X_{0}+Y_{0} \approx\right.$ $\sqrt{X_{0}}$ ) (for $\mathscr{R}_{3}, C_{1}$ can be inferred from (214)). For this we must relate $Q(0,0)$ to $C_{1}(\rho)$ by asymptotic matching, but this matching will require the analysis of another scale, which is intermediate to the $(X, Y)$ and $(n, p)$ scales. This analysis is carried out in the next subsection.

4.3. Analysis of the Scale $k=m-O\left(\rho^{1 / 3}\right), r=R-O\left(\rho^{2 / 3}\right)$. We will consider $m-k=v_{1} \rho^{1 / 3}=O\left(\rho^{1 / 3}\right)$ and $R-r=T_{1} \rho^{2 / 3}=$ $O\left(\rho^{2 / 3}\right)$. Then $X_{0}-X=O\left(\rho^{-2 / 3}\right)$ and $Y_{0}-Y=O\left(\rho^{-1 / 3}\right)$ so we are examining the vicinity of the corner point $\left(X_{0}, Y_{0}\right)$ along parabolas, where $\left(Y_{0}-Y\right)^{2} /\left(X_{0}-X\right)$ is constant. We note that the scale $k=m-O\left(\rho^{1 / 3}\right)$ (with $r=\rho Y$ and $Y>0$ ) was also important in the analysis of the model with $R=\infty$ (see [10]). There, a more geometric interpretation is given, in terms of caustic rays and caustic boundaries and also in terms of sample paths of large deviations. Since the caustic rays in region $\mathscr{D}_{\text {_ }}$ cannot fill the entire domain (for parameter regions $\mathscr{R}_{4}$ and $\mathscr{R}_{3} \cap \mathscr{R}_{4}$ ) we would expect a boundary effect near $Y=Y_{0}$ or $r=R$. The corner scale $k=m-O(1), r=R-O(1)$ that we analyzed in Section 4.2 is insufficient for fully understanding this boundary effect, and hence we analyze the scaling $r=R-O\left(\rho^{2 / 3}\right)$, which will connect the cases $r=R-O(1)$ and $r=\rho Y, Y<Y_{0}$. We cannot give an a priori probabilistic argument of why this scale is needed but mention that it leads to an interesting PDE, namely, (285), and such problems arise in many other areas, 
including queues with time-dependent arrival rates (see [20]) and steady two-dimensional convection-diffusion problems past curved obstacles (see [21]).

We first set

$$
Q(n, p)=\left(\sqrt{X_{0}}\right)^{n}\left(\frac{Y_{0}}{Y_{0}+W_{0}}\right)^{p} Q(n, p)
$$

with which the main balance equation (228) becomes

$$
\begin{aligned}
& {\left[1+X_{0}+Y_{0}-\rho^{-1}(n+p)\right] Q(n, p)} \\
& =\left[W_{0}+Y_{0}+\rho^{-1}(1-p) \frac{W_{0}+Y_{0}}{Y_{0}}\right] Q(n, p-1) \\
& \quad+\left[\sqrt{X_{0}}+\rho^{-1}(1-n) \frac{1}{\sqrt{X_{0}}}\right] Q(n-1, p) \\
& +\sqrt{X_{0}} \mathscr{Q}(n+1, p) .
\end{aligned}
$$

To obtain a limiting PDE on the $\left(\nu_{1}, T_{1}\right)$ scale, we first formally expand (277) using $Q(n \pm 1, p)=\mathbb{Q}(n, p) \pm \mathbb{Q}_{n}(n, p)+$ $(1 / 2) Q_{n n}(n+p)+\cdots$, where we anticipate that $n$ will be scaled to be large, with $n=O\left(\rho^{1 / 3}\right)$ and also $p=O\left(\rho^{2 / 3}\right)$. We thus rewrite (277) as

$$
\begin{aligned}
0= & \rho^{-1}\left[n+p+\frac{W_{0}+Y_{0}}{Y_{0}}(1-p)+\frac{1}{\sqrt{X_{0}}}(1-n)\right] \\
& \cdot \mathbb{Q}(n, p)-\left[W_{0}+Y_{0}+\rho^{-1} \frac{W_{0}+Y_{0}}{Y_{0}}(1-p)\right] \\
& \cdot \mathbb{Q}_{p}(n, p)+\frac{1}{2}\left(W_{0}+Y_{0}\right) \mathbb{Q}_{p p}(n, p)+\rho^{-1} \\
& \cdot \frac{1}{\sqrt{X_{0}}}(n-1) \mathbb{Q}_{n}(n, p)+\sqrt{X_{0}} \mathscr{Q}_{n n}(n, p)+\cdots,
\end{aligned}
$$

where the error term(s) involve derivatives of $\mathbb{Q}$ of order $\geqslant 3$ and terms of order $O\left(\rho^{-1} p Q_{p p}\right)$. We furthermore set

$$
\begin{aligned}
\mathcal{Q}(n, p) & =\exp \left[-\frac{W_{0}}{2 Y_{0}\left(W_{0}+Y_{0}\right)} \frac{p^{2}}{\rho}\right] \overline{\mathbb{Q}}(n, p) \\
& =\exp \left[-\frac{W_{0}}{2 Y_{0}\left(W_{0}+Y_{0}\right)} \rho^{1 / 3} T_{1}^{2}\right] \bar{Q}(n, p)
\end{aligned}
$$

so that

$$
\begin{aligned}
\mathcal{Q}_{p} & =\left(\frac{2 a p}{\rho} \bar{Q}+\bar{Q}_{p}\right) e^{a p^{2} / \rho}, \quad a=-\frac{W_{0}}{2 Y_{0}\left(W_{0}+Y_{0}\right)}, \\
\widehat{Q}_{p p} & =\left(\frac{4 a^{2} p^{2}}{\rho^{2}} \bar{Q}+\frac{4 a p}{\rho} \bar{Q}_{p}+\frac{2 a}{\rho} \bar{Q}+\bar{Q}_{p p}\right) e^{a p^{2} / \rho} \\
& \sim \frac{W_{0}^{2} T_{1}^{2}}{Y_{0}^{2}\left(W_{0}+Y_{0}\right)^{2}} \rho^{-2 / 3} \bar{Q} e^{a \rho^{1 / 3} T_{1}^{2}},
\end{aligned}
$$

where we isolated dominant term in (281) on the scale $p=$ $O\left(\rho^{2 / 3}\right)$. Setting $p=\rho^{2 / 3} T_{1}$ and $n=\rho^{1 / 3} v_{1}$, multiplying (278) by $\rho^{2 / 3} e^{-a \rho^{1 / 3} T_{1}^{2}}$, and using (279)-(281), we obtain the limiting PDE

$$
\begin{aligned}
\sqrt{X_{0}} \frac{\partial^{2} \bar{Q}}{\partial \nu_{1}^{2}}+\nu_{1}\left(1-\frac{1}{\sqrt{X_{0}}}\right) \bar{Q}-\left(W_{0}+Y_{0}\right) \frac{\partial \bar{Q}}{\partial T_{1}} \\
-\frac{W_{0}\left(W_{0}+2 Y_{0}\right)}{2 Y_{0}^{2}\left(W_{0}+Y_{0}\right)} T_{1}^{2} \bar{Q}=0,
\end{aligned}
$$

which applies over the quarter plane $\nu_{1}>0, T_{1}>0$, and we now view $\bar{Q}$ as a function of $\left(\nu_{1}, T_{1}\right)$ instead of $(n, p)$. By rescaling $\nu_{1}$ and $T_{1}$ using

$$
\begin{aligned}
& v_{1}=\left(\frac{X_{0}}{1-\sqrt{\mathrm{X}_{0}}}\right)^{1 / 3} \nu, \\
& T_{1}=\frac{\left(Y_{0}+W_{0}\right) X_{0}^{1 / 6}}{\left(1-\sqrt{X_{0}}\right)^{2 / 3}} T
\end{aligned}
$$

and letting

$$
\begin{gathered}
\bar{Q}\left(\nu_{1}, T_{1}\right)=\frac{\rho^{-2 / 3}\left(1-\sqrt{X_{0}}\right)^{5 / 3} Q(0,0)}{\left(\sqrt{X_{0}}-X_{0}-Y_{0}\right) X_{0}^{1 / 6}\left(W_{0}+Y_{0}\right)} \\
\cdot \exp \left[-\frac{T_{1}^{3}}{6} \frac{W_{0}\left(W_{0}+2 Y_{0}\right)}{Y_{0}^{2}\left(W_{0}+Y_{0}\right)^{2}}\right] \mathscr{F}(\nu, T)
\end{gathered}
$$

we obtain from (282) the separable, parabolic PDE

$$
\mathscr{F}_{\nu v}-v \mathscr{F}=\mathscr{F}_{T} ; \quad \nu>0, T>0 .
$$

Including the exponential factor in (284) allows us to eliminate the last term in the left-hand side of (282), while the other factors (such as $\rho^{-2 / 3}$ and $Q(0,0)$ ) are purely for convenience. We will show, by asymptotic matching between the $(n, p)$ and $(\nu, T)$ scales, that $\bar{Q}$ must be proportional to $Q(0,0)$, and including the factor $\rho^{-2 / 3}$ in (284) will lead to $\mathscr{F}$ being asymptotically $O(1)$.

Next we examine the boundary condition in (18), which can be also written as $\left(X_{0}+\rho^{-1}\right) P\left(X_{0}+\rho^{-1}, Y\right)=P\left(X_{0}, Y-\right.$ $\left.\rho^{-1}\right)$, or, using the $(n, p)$ variables, as

$$
\left(X_{0}+\rho^{-1}\right) Q(-1, p)=Q(0, p+1) \text {. }
$$

In view of (276) and (279) we can also write (286) as

$$
\begin{gathered}
\left(\sqrt{X_{0}}+\frac{1}{\sqrt{X_{0}}} \rho^{-1}\right) \mathscr{Q}(-1, p) \\
=\frac{Y_{0}}{Y_{0}+W_{0}} Q(0, p+1)
\end{gathered}
$$

or

$$
\begin{aligned}
& \left(\sqrt{X_{0}}+\frac{1}{\sqrt{X_{0}}} \rho^{-1}\right) \bar{Q}(-1, p)=\frac{Y_{0}}{Y_{0}+W_{0}} \\
& \cdot \exp \left[\frac{a}{\rho}(2 p+1)\right] \bar{Q}(0, p+1)=\frac{Y_{0}}{Y_{0}+W_{0}}[1 \\
& \left.-\frac{W_{0}}{Y_{0}\left(W_{0}+Y_{0}\right)} \rho^{-1 / 3} T_{1}+O\left(\rho^{-2 / 3}\right)\right] \bar{Q}(0, p+1),
\end{aligned}
$$

where we used also (280) and (284). 
On the $\left(\nu_{1}, T_{1}\right)$ scale we have

$$
\begin{aligned}
\bar{Q}(-1, p) & =\bar{Q}\left(-\rho^{-1 / 3}, T_{1}\right) \\
& =\bar{Q}\left(0, T_{1}\right)+O\left(\rho^{-1 / 3}\right), \\
\bar{Q}(0, p+1) & =\bar{Q}\left(0, T_{1}+\rho^{-2 / 3}\right) \\
& =\bar{Q}\left(0, T_{1}\right)+O\left(\rho^{-2 / 3}\right)
\end{aligned}
$$

so as long as $\sqrt{X_{0}} \neq Y_{0} /\left(Y_{0}+W_{0}\right)$ we conclude from (288) that $\bar{Q}$ must vanish along $v_{1}=0$ for all $T_{1}>0$, and thus, in view of (284) so must $\mathscr{F}$; hence

$$
\mathscr{F}(0, T)=0, \quad T>0 .
$$

The case where $\sqrt{X_{0}}=Y_{0} /\left(Y_{0}+W_{0}\right)$ corresponds to $\mathscr{R}_{3} \cap$ $\mathscr{R}_{4}$, and then the boundary condition will become more complicated. First we analyze the interior of $\mathscr{R}_{4}$ where (290) holds.

To analyze (285) with (290), we first derive the behavior of $\mathscr{F}$ as $\nu, T \rightarrow 0$, by matching the expansions on the $(n, p)$ and $(\nu, T)$ scales. To this end we need, for region $\mathscr{R}_{4}$, the behavior of $Q_{L}(n, p)$ for $n, p \rightarrow \infty$ with $n=O(\sqrt{p})$. This asymptotic limit lies in the asymptotic matching region between (249) (which applies for $0<n / p<\infty$ for region $\mathscr{R}_{4}$ ) and (253), which applies for $p \rightarrow \infty$ but with $n=O(1)$. Setting $u=$ $1 / \sqrt{X_{0}}+v / \sqrt{p}$ in the integral in (245) and shifting the contour $\Gamma$ toward $\operatorname{Re}(u)=1 / \sqrt{X_{0}}$ we obtain

$$
\begin{gathered}
Q_{L}(n, p) \sim Q(0,0)\left(\sqrt{X_{0}}\right)^{n}\left(\frac{Y_{0}}{W_{0}+Y_{0}}\right)^{p} \\
\cdot \frac{2 X_{0}^{3 / 2}\left(\sqrt{X_{0}-1}\right)}{p\left(W_{0}+Y_{0}\right)\left(X_{0}+Y_{0}-\sqrt{X_{0}}\right)} \frac{1}{2 \pi i} \\
\cdot \int_{-i \infty}^{i \infty} v \exp \left[-\frac{n}{\sqrt{p}} \sqrt{X_{0}} v+\frac{X_{0}^{3 / 2}}{W_{0}+Y_{0}} v^{2}\right] d v .
\end{gathered}
$$

Here we used

$$
u^{-n}=\left(\frac{1}{\sqrt{X_{0}}}+\frac{v}{\sqrt{p}}\right)^{-n} \sim X_{0}^{n / 2} \exp \left(-\frac{n}{\sqrt{p}} \sqrt{X_{0} v}\right)
$$

and approximated $\Gamma$ by a vertical contour. Evaluating the integral in (291) explicitly we conclude that

$$
\begin{aligned}
& Q_{L}(n, p) \sim \frac{Q(0,0)}{2 \sqrt{\pi}}\left(\sqrt{X_{0}}\right)^{n}\left(\frac{Y_{0}}{Y_{0}+W_{0}}\right)^{p} \\
& \cdot \frac{\left(1-\sqrt{X_{0}}\right) \sqrt{W_{0}+Y_{0}}}{X_{0}^{1 / 4}\left(\sqrt{X_{0}}-X_{0}-Y_{0}\right)} \frac{n}{p^{3 / 2}} \\
& \cdot \exp \left(-\frac{W_{0}+Y_{0}}{4 \sqrt{X_{0}}} \frac{n^{2}}{p}\right) ; \\
& n, p \longrightarrow \infty \text { with } n=O(\sqrt{p}) .
\end{aligned}
$$

By asymptotic matching the expansion on the $\left(\nu_{1}, T_{1}\right)$ scale must behave as the right side of (293), when $\nu_{1}, T_{1} \rightarrow 0$ with $v_{1} / \sqrt{T_{1}}$ fixed. But $n=\rho^{1 / 3} v_{1}, p=\rho^{2 / 3} T_{1}$, and (283) shows that

$$
\begin{aligned}
\frac{n^{2}}{p} & =\frac{v_{1}^{2}}{T_{1}}=\frac{\sqrt{X_{0}}}{W_{0}+Y_{0}} \frac{v^{2}}{T}, \\
\frac{n}{p^{3 / 2}} & =\frac{\left(1-\sqrt{X_{0}}\right)^{2 / 3} X_{0}^{1 / 12}}{\rho^{2 / 3}\left(W_{0}+Y_{0}\right)^{3 / 2}} \frac{v}{T^{3 / 2}} .
\end{aligned}
$$

Then comparing (293) with (276) (using (279) and (284)) we conclude that

$$
\begin{aligned}
& \mathscr{F}(\nu, T) \sim \frac{v}{2 \sqrt{\pi} T^{3 / 2}} \exp \left(-\frac{\nu^{2}}{4 T}\right) ; \\
& \nu, T \longrightarrow 0 \text { with } \nu=O(\sqrt{T}) .
\end{aligned}
$$

Note that (295) is consistent with the boundary condition in (290) along $v=0$. The exponential factors in (279) and (284) do not enter the matching condition, since $T_{1}=O(1)$ and $\rho^{1 / 3} T_{1}^{2}=\rho^{-1} p^{2}$, and we can choose $p$ so that $p=o\left(\rho^{1 / 2}\right)$ in the matching region.

To solve (285) subject to (295) it is useful to view the function $(\pi T)^{-1 / 2} \exp \left[-\nu^{2} /(4 T)\right]$ as being an approximation to the delta function $\delta(v)$ for $T \rightarrow 0$, with the mass concentrated in the range $v>0$. Then the right side of (295) corresponds to the dipole $-\delta^{\prime}(v)$. Consider the problem

$$
\begin{gathered}
\mathscr{F}_{\nu v}^{*}-v \mathscr{F}^{*}=\mathscr{F}_{T}^{*} ; \quad v>0, T>0 \\
\mathscr{F}^{*}(\nu, 0)=\delta\left(v-v_{0}\right), \quad v_{0}>0 \\
\mathscr{F}^{*}(0, T)=0, \quad T>0 .
\end{gathered}
$$

Introducing the Laplace transform

$$
\widehat{\mathscr{F}}=\widehat{\mathscr{F}}\left(\nu, \theta ; \nu_{0}\right)=\int_{0}^{\infty} e^{-\theta T} \mathscr{F}^{*}\left(\nu, T ; v_{0}\right) d T,
$$

(296) becomes

$$
\widehat{\mathscr{F}}_{\nu \nu}-(\nu+\theta) \widehat{\mathscr{F}}=-\delta\left(\nu-v_{0}\right), \quad v>0
$$

and (298) implies that $\widehat{\mathscr{F}}(0, \theta)=0$. The problem in (300) is a standard Green's function problem with solution

$$
\begin{aligned}
\widehat{\mathscr{F}} & =-\pi \frac{\operatorname{Ai}\left(\nu_{0}+\theta\right)}{\operatorname{Ai}(\theta)}[\operatorname{Ai}(\nu+\theta) \operatorname{Bi}(\theta) \\
& -\operatorname{Bi}(\nu+\theta) \operatorname{Ai}(\theta)], \quad 0<\nu<v_{0}, \\
\widehat{\mathscr{F}} & =-\pi \frac{\operatorname{Ai}(\nu+\theta)}{\operatorname{Ai}(\theta)}\left[\operatorname{Ai}\left(\nu_{0}+\theta\right) \operatorname{Bi}(\theta)\right. \\
& \left.-\operatorname{Bi}\left(v_{0}+\theta\right) \operatorname{Ai}(\theta)\right], \quad v>v_{0},
\end{aligned}
$$

where $\operatorname{Ai}(\cdot)$ and $\operatorname{Bi}(\cdot)$ are the Airy functions. Then the solution $\mathscr{F}$ to (285) will be

$$
\mathscr{F}(\nu, T)=\left.\frac{\partial}{\partial \nu_{0}} \mathscr{F}\left(\nu, T ; \nu_{0}\right)\right|_{\nu_{0}=0} .
$$


By differentiating (302) with respect to $\nu_{0}$, setting $\nu_{0}=0$, and noting the Wronskian identity $\operatorname{Ai}(\theta) \operatorname{Bi}^{\prime}(\theta)-\operatorname{Ai}^{\prime}(\theta) \operatorname{Bi}(\theta)=\pi^{-1}$ we find that

$$
\left.\frac{\partial \widehat{\mathscr{F}}}{\partial \nu_{0}}\right|_{\nu_{0}=0}=\frac{\operatorname{Ai}(\nu+\theta)}{\operatorname{Ai}(\theta)}, \quad v>0 .
$$

Inverting the Laplace transform in (299) leads to $\mathscr{F}(\nu, T)$ in (170), so we have established the expansion on the $\left(\nu_{1}, T_{1}\right)$ scale. We can also easily verify that (295) is indeed satisfied, since

$$
\begin{aligned}
\mathscr{F} & (\nu, T)=\frac{1}{2 \pi i} \frac{d}{d T} \int_{-i \infty}^{i \infty} \frac{\operatorname{Ai}(\nu+\theta)}{\operatorname{Ai}(\theta)} e^{\theta T} d \theta \\
& \sim \frac{1}{2 \pi i} \frac{d}{d T} \int_{-i \infty}^{i \infty} \frac{e^{\theta T-\nu \sqrt{\theta}}}{\theta}\left[1+O\left(\frac{\nu^{2}}{\sqrt{\theta}}\right)\right] d \theta \\
\sim & \frac{1}{\pi i} \int_{-i \infty}^{i \infty} e^{\omega^{2} T-\omega \nu} \omega d \omega \\
& =\frac{v}{2 \sqrt{\pi} T^{3 / 2}} \exp \left(-\frac{\nu^{2}}{4 T}\right) .
\end{aligned}
$$

Here we approximated the first integrand for $\theta \rightarrow \infty$ using asymptotic properties of $\mathrm{Ai}(\cdot)$.

We next relate the constant $C_{1}$ in $\mathscr{D}_{-}$to $Q(0,0)(\sim 1-$ $X_{0}-Y_{0}$ in $\left.\mathscr{R}_{4}\right)$, having expressed the expansions near the corner point $\left(X_{0}, Y_{0}\right)$ in terms of $Q(0,0)$ on both the $(n, p)$ and $\left(\nu_{1}, T_{1}\right)$ scales. As we discussed previously, the caustic ray expansion in $\mathscr{D}_{-}$does not satisfy the boundary condition along $X=X_{0}$, and different expansions must be constructed on the $\nu_{1}$ and $n$ scales, corresponding to $X_{0}-X=O\left(\rho^{-2 / 3}\right)$ and $X_{0}-X=O\left(\rho^{-1}\right)$. For region $\mathscr{R}_{4}$, these expansions are given, respectively, in (111) and (109) (with (110)), up to the constant $C_{1}$. The details of their construction are given in [10]. We will need to carefully estimate $\pi(k, r)$ for $n=m-k=O(1)$. For region $\mathscr{R}_{4},(109)$ applies for $n=O(1)$ and all $Y \in\left(0, Y_{0}\right)$ (but breaks down as either $Y \downarrow 0$ or $\left.Y \uparrow Y_{0}\right)$. For $n=O(1)$ and $p=O(1),(241)$ holds as then $\pi(k, r) \sim Q_{L}(n, p)$, and in (253) we gave the asymptotics for $Q(n, p)$ for $p \rightarrow \infty$ and $n=O(1)$. However, we must derive yet another expansion on the scale $T_{1}=O(1)\left(Y_{0}-Y=O\left(\rho^{-1 / 3}\right)\right)$ with $T_{1}>0$ and $n=O(1)$. The expansion in (169) develops a nonuniformity as $v \rightarrow 0$, since $\mathscr{F}(0, T)=0$. Since by definition of the Airy roots $r_{j}$ we have $\operatorname{Ai}\left(r_{j}\right)=0$, from the infinite series form in (170) we conclude that

$$
\begin{array}{r}
\mathscr{F}(\nu, T) \sim \nu \sum_{j=0}^{\infty} e^{r_{j} T}=\left(\frac{1-\sqrt{X_{0}}}{X_{0}}\right)^{1 / 3} \frac{n}{\rho^{1 / 3}} \sum_{j=0}^{\infty} e^{-\left|r_{j}\right| T}, \\
v \longrightarrow 0,
\end{array}
$$

and this holds for any fixed $T>0$. Consider the scale $n=O(1)$ and $T_{1}>0$. Viewing $Q$ in (277) now as a function of $n$ and $T_{1}$ and noting that $p \rightarrow p \pm 1$ corresponds to $T_{1} \rightarrow T_{1} \mp \rho^{-2 / 3}$ the limiting form of (277) becomes

$$
\begin{aligned}
0= & \left(W_{0}-1-X_{0}\right) \mathbb{Q}\left(n, T_{1}\right) \\
& +\sqrt{X_{0}}\left[\mathcal{Q}\left(n-1, T_{1}\right)+\mathscr{Q}\left(n+1, T_{1}\right)\right] .
\end{aligned}
$$

But $W_{0}=\left(1-\sqrt{X_{0}}\right)^{2}=1+X_{0}-2 \sqrt{X_{0}}$ so that the general solution of (307) is the linear function $a\left(T_{1}\right)+n b\left(T_{1}\right)$, where $a(\cdot)$ and $b(\cdot)$ are arbitrary functions of $T_{1}$. Note also that (307) applies asymptotically whether the exponential factors in (279) and (284) are included or excluded. The boundary condition in (287) can be applied on the $n=O(1)$ scale and leads asymptotically to $\left(W_{0}+Y_{0}\right) \sqrt{X_{0}} Q\left(-1, T_{1}\right)=Y_{0} Q\left(0, T_{1}\right)$. This restricts the functions $a(\cdot)$ and $b(\cdot)$ in the linear solution to (307), by

$$
Y_{0} a\left(T_{1}\right)=\left(W_{0}+Y_{0}\right) \sqrt{X_{0}}\left[a\left(T_{1}\right)-b\left(T_{1}\right)\right] .
$$

We have thus shown that the expansion on the $\left(n, T_{1}\right)$ scale is given by

$$
\begin{aligned}
& \pi(k, r) \sim Q(0,0) \rho^{-1} \\
& \cdot \frac{\left(1-\sqrt{X_{0}}\right)^{2}}{\left(W_{0}+Y_{0}\right) \sqrt{X_{0}}\left(\sqrt{X_{0}}-X_{0}-Y_{0}\right)}\left(\sqrt{X_{0}}\right)^{n} \\
& \cdot\left(\frac{Y_{0}}{Y_{0}+W_{0}}\right)^{p}\left[n+\frac{\sqrt{X_{0}}\left(Y_{0}+W_{0}\right)}{\left(Y_{0}+W_{0}\right) \sqrt{X_{0}}-Y_{0}}\right] f_{*}(T) \\
& \cdot \exp \left[-\frac{W_{0}}{2 Y_{0}\left(W_{0}+Y_{0}\right)} \rho^{1 / 3} T_{1}^{2}\right. \\
& \left.-\frac{W_{0}\left(W_{0}+2 Y_{0}\right)}{Y_{0}\left(W_{0}+Y_{0}\right)^{2}} T_{1}^{3}\right] .
\end{aligned}
$$

Here we made $a(\cdot)$ proportional to $f_{*}(\cdot)$, which we will determine shortly, and computed $b(\cdot)$ from (308). Now we require (309), for $n \rightarrow \infty$, to match to the $\left(\nu_{1}, T_{1}\right)$ scale result, for $v_{1} \rightarrow 0$. But in view of (306) we see that the matching is possible provided that

$$
f_{*}(T)=\sum_{j=0}^{\infty} e^{-\left|r_{j}\right| T}
$$

Thus we now have three expansions valid for $n=O(1)$, on the scales $p=O(1), p=O\left(\rho^{2 / 3}\right)$, and $p=O(\rho)$ with $Y<Y_{0}$. The matching between the $p=O(1)$ result and (309) follows by letting $p \rightarrow \infty$ in (241) (corresponding to (253) for region $\left.\mathscr{R}_{4}\right)$ and letting $T \rightarrow 0$. The matching condition is satisfied if

$$
\begin{aligned}
& \frac{1}{2 \sqrt{\pi}} p^{-3 / 2} \frac{\sqrt{W_{0}+Y_{0}}\left(1-\sqrt{X_{0}}\right)}{X_{0}^{1 / 4}\left(\sqrt{X_{0}}-X_{0}-Y_{0}\right)} \\
& \sim \frac{\left(1-\sqrt{X_{0}}\right)^{2} \rho^{-1}}{\left(W_{0}+Y_{0}\right) \sqrt{X_{0}}\left(\sqrt{X_{0}}-X_{0}-Y_{0}\right)} f_{*}(T),
\end{aligned}
$$

in some intermediate limit where $p \rightarrow \infty$ and $T_{1}=\rho^{-2 / 3} p \rightarrow$ 0 . Simplifying (311) by using (283) we must show that

$$
f_{*}(T) \sim \frac{1}{2 \sqrt{\pi}} T^{-3 / 2}, \quad T \longrightarrow 0 .
$$


The Airy roots $r_{j}$ are well known to satisfy $-r_{j} \sim(3 j \pi / 2)^{2 / 3}$, $j \rightarrow \infty$, so for $T \rightarrow 0$ we approximate the sum in (310) by an integral to get

$$
\begin{aligned}
\sum_{j=0}^{\infty} e^{-\left|r_{j}\right| T} & \sim \int_{0}^{\infty} \exp \left[-\left(\frac{3}{2} \xi \pi\right)^{2 / 3} T\right] d \xi \\
& =\frac{2}{3 \pi} T^{-3 / 2}\left(\int_{0}^{\infty} e^{-u} \frac{3}{2} \sqrt{u} d u\right) \\
& =\frac{1}{\pi} T^{-3 / 2} \Gamma\left(\frac{3}{2}\right)
\end{aligned}
$$

which verifies (312).

Now we match (309) for $T \rightarrow \infty$ with (109) as $Y \rightarrow Y_{0}$. For $T \rightarrow \infty$ we have $f_{*}(T) \sim e^{-\left|r_{0}\right| T}$ as the zeroth term in the sum in (310) dominates. By expanding (109) for $Y \rightarrow Y_{0}$, after canceling some common factors the matching implies that

$$
\begin{aligned}
& \frac{\rho^{-1} Q(0,0) W_{0}}{\left(W_{0}+Y_{0}\right) \sqrt{X_{0}}\left(\sqrt{X_{0}}-X_{0}-Y_{0}\right)}\left(\frac{Y_{0}}{Y_{0}+W_{0}}\right)^{p} e^{r T} \\
& \cdot \exp \left[-\frac{W_{0}}{2 Y_{0}\left(W_{0}+Y_{0}\right)} \rho^{1 / 3} T_{1}^{2}\right. \\
& \left.-\frac{W_{0}\left(W_{0}+2 Y_{0}\right)}{Y_{0}^{2}\left(W_{0}+Y_{0}\right)^{2}} T_{1}^{3}\right] \sim \rho^{-1 / 6} C_{1}(\rho) \\
& \cdot e^{\rho \Phi\left(X_{0}, Y_{0}\right)} e^{\rho \Phi_{Y}\left(X_{0}, Y_{0}\right)\left(Y-Y_{0}\right)} e^{\rho^{1 / 3} \Phi_{*}\left(Y_{0}\right)} \\
& \cdot \exp \left[\frac{1}{2} \rho \Phi_{Y Y}\left(X_{0}, Y_{0}\right)\left(Y-Y_{0}\right)^{2}\right. \\
& +\frac{1}{6} \rho \Phi_{Y Y Y}\left(X_{0}, Y_{0}\right)\left(Y-Y_{0}\right)^{3} \\
& \left.+\rho^{1 / 3} \Phi_{*}^{\prime}\left(Y_{0}\right)\left(Y-Y_{0}\right)\right] \\
& \cdot \exp \left[-\frac{1+\sqrt{X_{0}}}{2 \sqrt{X_{0}}} \log \left(\frac{Y_{0}+W_{0}}{1-\sqrt{X_{0}}}\right)\right] \frac{1}{\sqrt{2 \pi}} \frac{1}{\operatorname{Ai}^{\prime}\left(r_{0}\right)} \\
& \cdot \frac{\left(Y_{0}+W_{0}\right) \sqrt{X_{0}}-Y_{0}}{\sqrt{Y_{0}} X_{0}^{5 / 6}\left(1-\sqrt{X_{0}}\right)^{1 / 6}} \text {. }
\end{aligned}
$$

Now,

$$
\begin{gathered}
e^{\rho \Phi_{Y}\left(X_{0}, Y_{0}\right)\left(Y-Y_{0}\right)}=e^{-p \Phi_{Y}\left(X_{0}, Y_{0}\right)}=\left(\frac{Y_{0}}{Y_{0}+W_{0}}\right)^{p} \\
\exp \left[\rho^{1 / 3} \Phi_{*}^{\prime}\left(Y_{0}\right)\left(Y-Y_{0}\right)\right]=\exp \left[-T_{1} \Phi_{*}^{\prime}\left(Y_{0}\right)\right] \\
\quad=\exp \left[r_{0} T_{1} \frac{\left(1-\sqrt{X_{0}}\right)^{2 / 3}}{\left(W_{0}+Y_{0}\right) X_{0}^{1 / 6}}\right]=e^{r_{0} T} .
\end{gathered}
$$

By using (110) to compute $\Phi_{Y Y}$ and $\Phi_{Y Y Y}$ and noting that

$$
\begin{aligned}
& \rho\left(Y-Y_{0}\right)^{2}=\rho^{1 / 3} T_{1}^{2}, \\
& \rho\left(Y-Y_{0}\right)^{3}=-T_{1}^{3}
\end{aligned}
$$

we see that the dependence on $T_{1}$ is exactly the same in the left- and right-hand sides of (314). Thus we have a relation between the constants $Q(0,0)$ and $C_{1}$ for region $\mathscr{R}_{4}$, and since $Q(0,0) \sim 1-X_{0}-Y_{0}$ we have derived the expression for $C_{1}$ in (41).

The analysis for region $\mathscr{R}_{4}$ breaks down as $Y_{0} \rightarrow \sqrt{X_{0}}-$ $X_{0}$, as then the expressions in (284), (293), (309), and (314) all become singular. We thus examine the transitional case $\mathscr{R}_{3} \cap \mathscr{R}_{4}$, where $X_{0}+Y_{0}=\sqrt{X_{0}}+O\left(\rho^{-1 / 3}\right)$. Then we certainly have $Q(0,0) \sim 1-X_{0}-Y_{0}$ and (241) applies for any $\mathscr{R}_{j}$. But now on parabolic scales, where $n, p \rightarrow \infty$ with $n^{2} / p$ fixed, $Q(n, p)$ has the expansion in (254). We now define $\overline{\mathscr{F}}(\nu, T)$ by setting

$$
\begin{gathered}
\pi(k, r) \sim Q(0,0) \rho^{-1 / 3}\left(\frac{1-\sqrt{X_{0}}}{X_{0}}\right)^{1 / 3}\left(\sqrt{X_{0}}\right)^{n} \\
\cdot\left(\frac{Y_{0}}{Y_{0}+W_{0}}\right)^{p} \exp \left[-\frac{W_{0}}{2 Y_{0}\left(W_{0}+Y_{0}\right)} \rho^{1 / 3} T_{1}^{2}\right. \\
\left.-\frac{W_{0}\left(2 Y_{0}+W_{0}\right)}{6 Y_{0}^{2}\left(W_{0}+Y_{0}\right)^{2}} T_{1}^{3}\right] \overline{\mathscr{F}}(\nu, T)
\end{gathered}
$$

since the factors in (317) correspond to the same sequence of transformations we made for the analysis of the interior of $\mathscr{R}_{4}$, and $\overline{\mathscr{F}}$ will again satisfy the PDE

$$
\overline{\mathscr{F}}_{\nu v}-\rho \overline{\mathscr{F}}=\overline{\mathscr{F}}_{T} ; \quad v>0, T>0 .
$$

Note that in (317) we included the factor $\rho^{-1 / 3}$ whereas in (284) we have the two factors $\rho^{-2 / 3}$ and $\left[\sqrt{X_{0}}-X_{0}-Y_{0}\right]^{-1}$, as the product of these becomes, in region $\mathscr{R}_{3} \cap \mathscr{R}_{4}, O\left(\rho^{-2 / 3}\right) \times$ $O\left(\rho^{1 / 3}\right)=O\left(\rho^{-1 / 3}\right)$. Including the factor $\rho^{-1 / 3}$ will insure that $\overline{\mathscr{F}}$ is $O(1)$ for $\rho \rightarrow \infty$, and the factor $\left(1-\sqrt{X_{0}}\right)^{1 / 3} X_{0}^{-1 / 3}$ is purely for convenience. But for region $\mathscr{R}_{3} \cap \mathscr{R}_{4}$ we must reexamine the boundary condition in (288), which in terms of $\overline{\mathscr{F}}$ becomes

$$
\begin{aligned}
& {\left[\sqrt{X_{0}}+O\left(\rho^{-1}\right)\right] \overline{\mathscr{F}}\left(-\rho^{1 / 3}, T_{1}\right)=\frac{Y_{0}}{Y_{0}+W_{0}}[1} \\
& \left.\quad-\frac{W_{0}}{Y_{0}\left(W_{0}+Y_{0}\right)} \rho^{-1 / 3} T_{1}+O\left(\rho^{-2 / 3}\right)\right] \overline{\mathscr{F}}\left(0, T_{1}\right. \\
& \left.\quad+\rho^{-2 / 3}\right) .
\end{aligned}
$$

But now

$$
\begin{aligned}
\left(Y_{0}\right. & \left.+W_{0}\right) \sqrt{X_{0}}-Y_{0} \\
& =\left(1-\sqrt{X_{0}}\right)\left(\sqrt{X_{0}}-X_{0}-Y_{0}\right) \\
& =-\left(1-\sqrt{X_{0}}\right) \delta_{*} \rho^{-1 / 3}
\end{aligned}
$$

so that the limiting boundary condition from (319) becomes

$$
\sqrt{X_{0}} \frac{\partial \overline{\mathscr{F}}}{\partial \nu_{1}}+\left(\delta_{*}-T_{1}\right) \overline{\mathscr{F}}=0, \quad v_{1}=0
$$


Then scaling $\delta_{*}=X_{0}^{1 / 6}\left(1-\sqrt{X_{0}}\right)^{1 / 3} \delta_{1}$, in terms of $(\nu, T)(321)$ becomes

$$
\overline{\mathscr{F}}_{\nu}(0, T)+\left(\delta_{1}-T\right) \overline{\mathscr{F}}(0, T)=0, \quad T>0 .
$$

A matching condition for $\overline{\mathscr{F}}$, as $v, T \rightarrow 0$ with $\nu=O(\sqrt{T})$, is obtained by comparing (317) to (254). From the definitions of $\delta$ and $\delta_{*}$ we have $\delta \rho^{-1 / 2}=\delta_{*} \rho^{-1 / 3}$ so that $\delta=\delta_{*} \rho^{1 / 6}$. Then also

$$
\begin{aligned}
\frac{p \delta^{2}}{\rho} & =T_{1} \delta_{*}^{2}, \\
\frac{n \delta}{\sqrt{\rho}} & =v_{1} \delta_{*}, \\
\frac{n^{2}}{p} & =\frac{v_{1}^{2}}{T_{1}}
\end{aligned}
$$

are all $O(1)$, while $p^{-1 / 2}=\rho^{-1 / 3} T_{1}^{-1 / 2}$ and $\delta \rho^{-1 / 2}=\delta_{*} \rho^{-1 / 3}$, so in the matching region, where $T_{1}$ is small but $\delta_{*}=O(1)$, the Gaussian first term in the braces in (254) dominates the error function second term, and the matching will imply that

$$
\begin{aligned}
& \left(\frac{1-\sqrt{X_{0}}}{X_{0}}\right)^{1 / 3} \overline{\mathscr{F}}(v, T) \\
& \sim \frac{1}{\sqrt{\pi}} \frac{\left(1-\sqrt{X_{0}}\right)^{1 / 2}}{X_{0}^{1 / 4} \sqrt{T_{1}}} \exp \left(-\frac{1-\sqrt{X_{0}}}{4 \sqrt{X_{0}}} \frac{v_{1}^{2}}{T_{1}}\right)
\end{aligned}
$$

and in terms of $(\nu, T)$ this simplifies to

$$
\begin{aligned}
\overline{\mathscr{F}}(\nu, T) \sim \frac{1}{\sqrt{\pi T}} \exp \left(-\frac{\nu^{2}}{4 T}\right) ; \\
v, T \longrightarrow 0 \text { with } \nu=O(\sqrt{T}) .
\end{aligned}
$$

Here we also used $Y_{0}+W_{0} \sim 1-\sqrt{X_{0}}$ in region $\mathscr{R}_{3} \cap \mathscr{R}_{4}$, so that $T_{1} \sim\left(1-\sqrt{X_{0}}\right)^{1 / 3} X_{0}^{1 / 6} T$.

The matching condition in (325) may be replaced by the condition $\left.\overline{\mathscr{F}}\right|_{T=0}=\delta(\nu)$ and we analyzed such problems in [20] where it was found that the solution to (318) with (322) is given by

$$
\begin{aligned}
& \bar{F}(\nu, T)=\frac{1}{2 \pi i} \\
& \cdot \int_{-i \infty}^{i \infty} \frac{\operatorname{Ai}(\nu+\theta)}{\operatorname{Ai}^{2}(\theta)} e^{\theta\left(T-\delta_{1}\right)}\left(\int_{\theta}^{\infty} e^{\delta_{1} u} \operatorname{Ai}(u) d u\right) d \theta .
\end{aligned}
$$

The right-hand side of (326) clearly satisfies (318), since this PDE is separable and any function of the form $g(\theta) \operatorname{Ai}(\nu+$ $\theta) e^{\theta T}$ is a solution, for any function $g(\cdot)$ and any complex $\theta$. We can also verify that (322) holds along $\nu=0$, by using

$$
\begin{aligned}
& \frac{\operatorname{Ai}^{\prime}(\theta)}{[\operatorname{Ai}(\theta)]^{2}} e^{\theta\left(T-\delta_{1}\right)}+\frac{\delta_{1}-T}{\operatorname{Ai}(\theta)} e^{\theta\left(T-\delta_{1}\right)} \\
& =-\frac{d}{d \theta}\left[\frac{e^{\theta\left(T-\delta_{1}\right)}}{\operatorname{Ai}(\theta)}\right]
\end{aligned}
$$

and some integration by parts in (326). To verify the matching condition in (325) we expand the integrand in (326) for $v \rightarrow$ $0, T \rightarrow 0$, and $\theta \rightarrow \infty$, using (190) to approximate the Airy function. We have $\operatorname{Ai}(\nu+\theta) / \operatorname{Ai}(\theta) \sim e^{-v \sqrt{\theta}}$ and

$$
\begin{aligned}
& e^{-\theta \delta_{1}} \int_{\theta}^{\infty} e^{\delta_{1} u} \operatorname{Ai}(u) d u=\int_{0}^{\infty} e^{\delta_{1} \xi} \operatorname{Ai}(\theta+\xi) d \xi \\
& \sim e^{-(2 / 3) \theta^{3 / 2}} \frac{1}{\sqrt{4 \pi}} \int_{0}^{\infty} \exp \left[-\sqrt{\theta} \xi+O\left(\frac{\xi^{2}}{\sqrt{\theta}}\right)\right] \\
& \cdot\left[1+\delta_{1} \xi+O\left(\xi^{2}\right)\right] \\
& \cdot \theta^{-1 / 4}\left[1-\frac{\xi}{4 \theta}+O\left(\frac{\xi^{2}}{\theta^{2}}\right)\right] d \xi=e^{-(2 / 3) \theta^{3 / 2}} \\
& \cdot \frac{1}{\sqrt{4 \pi}} \theta^{-1 / 4}\left[\frac{1}{\sqrt{\theta}}+\frac{\delta_{1}}{\theta}+o\left(\theta^{-1}\right)\right]=\operatorname{Ai}(\theta)\left[\frac{1}{\sqrt{\theta}}\right. \\
& \left.+\frac{\delta_{1}}{\theta}+o\left(\theta^{-1}\right)\right] .
\end{aligned}
$$

Here we also expanded the integrand for small $\xi$, since by Watson's lemma the main contribution comes from the range $\xi=O\left(\theta^{-1 / 2}\right)$. Using (328) in (326) and noting that

$$
\begin{aligned}
& \frac{1}{2 \pi i} \int_{-i \infty}^{i \infty} e^{\theta T-\sqrt{\theta} \nu} \frac{d \theta}{\sqrt{\theta}}=\frac{1}{\sqrt{\pi T}} \exp \left(-\frac{\nu^{2}}{4 T}\right), \\
& \frac{1}{2 \pi i} \int_{-i \infty}^{i \infty} e^{\theta T-\sqrt{\theta} \nu} \frac{d \theta}{\theta}=\frac{1}{\sqrt{2 \pi}} \int_{\nu / \sqrt{2 T}}^{\infty} e^{-u^{2} / 2} d u
\end{aligned}
$$

we obtain, for $v, T \rightarrow 0$ with $v=O(\sqrt{T})$,

$$
\begin{aligned}
\overline{\mathscr{F}}(\nu, T)= & \frac{1}{\sqrt{\pi T}} \exp \left(-\frac{\nu^{2}}{4 T}\right) \\
& +\sqrt{\frac{2}{\pi}} \delta_{1} \int_{\nu / \sqrt{2 T}}^{\infty} e^{-u^{2} / 2} d u+o(1) .
\end{aligned}
$$

The expression in (330) is a two-term asymptotic approximation in this limit, and the leading term verifies the matching condition in (325). The correction term in (330), which is proportional to $\delta_{1}$, will asymptotically match to the term proportional to the error function in (254), as in the matching region $p \delta^{2} / \rho=T_{1} \delta_{*}^{2}$ and $n \delta / \sqrt{\rho}=\nu_{1} \delta_{*}$ are $o(1)$, and

$$
\begin{aligned}
\eta_{0} & \sim \frac{\left(1-\sqrt{X_{0}}\right)^{1 / 2}}{\sqrt{2} X_{0}^{1 / 4}} \frac{\nu_{1}}{\sqrt{T_{1}}} \sim \frac{v}{\sqrt{2 T}}, \\
\sqrt{\frac{2}{X_{0}}} \frac{\delta}{\sqrt{\rho}} & =\rho^{-1 / 3}\left(\frac{1-\sqrt{X_{0}}}{X_{0}}\right)^{1 / 3} \sqrt{2} \delta_{1} .
\end{aligned}
$$

We proceed to determine $C_{1}$. Now the approximation in (209) (with (326)) remains valid on the scale $n=O(1)$, since $\overline{\mathscr{F}}(0, T) \neq 0$. Thus for $n=O(1) \pi(k, r)$ can be approximated by (317) with $\overline{\mathscr{F}}(\nu, T)$ replaced by $\overline{\mathscr{F}}(0, T)$ and for $T \rightarrow \infty$ 
the expansion of (326) is determined by the pole at $\theta=r_{0}<0$, which is a simple pole if $v=0$. Hence,

$$
\begin{aligned}
\overline{\mathscr{F}}(0, T) \sim \frac{e^{r_{0}\left(T-\delta_{1}\right)}}{\operatorname{Ai}^{\prime}\left(r_{0}\right)}\left(\int_{r_{0}}^{\infty} e^{\delta_{1} u} \operatorname{Ai}(u) d u\right), & \\
& T \longrightarrow \infty .
\end{aligned}
$$

For $n=O(1)$ and $0<Y<Y_{0}$, the result in (109) holds, and this remains finite as $Y \uparrow Y_{0}$ even if $X_{0}+Y_{0}=\sqrt{X_{0}}$. In parameter region $\mathscr{R}_{3} \cap \mathscr{R}_{4}$ we have $Y_{0}+W_{0} \sim 1-\sqrt{X_{0}}$ and then as $Y \uparrow Y_{0}$, (109) becomes

$$
\begin{aligned}
& \rho^{-1 / 6} C_{1}(\rho)\left(\sqrt{X_{0}}\right)^{n} \\
& \cdot e^{\rho \Phi\left(X_{0}, Y_{0}\right)} e^{-p \Phi_{Y}\left(X_{0}, Y_{0}\right)} e^{\rho^{1 / 3} \Phi_{*}\left(Y_{0}\right)} e^{r_{0} T} \\
& \cdot \exp \left[\frac{1}{2} \rho^{1 / 3} \Phi_{Y Y}\left(X_{0}, Y_{0}\right) T_{1}^{2}\right. \\
& \left.-\frac{1}{6} \Phi_{Y Y Y}\left(X_{0}, Y_{0}\right) T_{1}^{3}\right] \frac{1}{\sqrt{2 \pi}} \frac{1}{\mathrm{Ai}^{\prime}\left(r_{0}\right)}(1 \\
& \left.-\sqrt{X_{0}}\right)^{1 / 3} X_{0}^{-7 / 12} .
\end{aligned}
$$

Comparing (333) to (317) with (332), the exponential parts agree as for region $\mathscr{R}_{4}$, and we now have

$$
\begin{aligned}
& \rho^{1 / 3} \Phi_{*}\left(Y_{0}\right) \\
& =-\rho^{1 / 3} r_{0} \frac{\left(1-\sqrt{X_{0}}\right)^{2 / 3}}{X_{0}^{1 / 6}} \log \left(\frac{Y_{0}+W_{0}}{1-\sqrt{X_{0}}}\right) \\
& =-\rho^{1 / 3} r_{0} \frac{\left(1-\sqrt{X_{0}}\right)^{2 / 3}}{X_{0}^{1 / 6}} \log \left(1+\frac{\rho^{-1 / 3} \delta_{*}}{1-\sqrt{X_{0}}}\right) \\
& \sim-r_{0}\left(1-\sqrt{X_{0}}\right)^{-1 / 3} X_{0}^{-1 / 6} \delta_{*}=-r_{0} \delta_{1} .
\end{aligned}
$$

Thus (333) agrees with (317), with $\nu=0$ and $T \rightarrow \infty$, if

$$
\begin{gathered}
Q(0,0) \rho^{-1 / 3} X_{0}^{-1 / 3}\left(\int_{r_{0}}^{\infty} e^{\delta_{1} u} \operatorname{Ai}(u) d u\right) \\
\sim \frac{C_{1}(\rho)}{\sqrt{2 \pi}} \rho^{-1 / 6} X_{0}^{-7 / 12} e^{\rho \Phi\left(X_{0}, Y_{0}\right)} .
\end{gathered}
$$

But now $Q(0,0) \sim 1-X_{0}-Y_{0} \sim 1-\sqrt{X_{0}}$ and thus (335) leads to the result in (37).

We have now determined the values of $Q(0,0), C_{0}(\rho)$, $C(\rho)$, and $C_{1}(\rho)$ for all possible regions $\mathscr{R}_{j}$ of parameter space, thus establishing Propositions 1 and 2 . We also have analyzed all of the relevant scales near the corner point $\left(X_{0}, Y_{0}\right)$ of the state space and thus established all parts of Proposition 15. As a final step we briefly discuss the asymptotic matching between the ray expansion in $\mathscr{D}_{0}$ and the expansion on the $(\nu, T)$ scale in $(169)$. We only consider region $\mathscr{R}_{4}$ and note that this matching will involve letting
$(X, Y) \rightarrow\left(X_{0}, Y_{0}\right)$ along parabolic scales with $\left(Y_{0}-Y\right)^{2} /\left(X_{0}-\right.$ $X)\left(=T_{1}^{2} / \nu_{1}\right)$ held fixed and letting $\nu_{1}, T_{1} \rightarrow \infty$ in (169). We have already verified that along linear scales, where $(X, Y) \rightarrow$ $\left(X_{0}, Y_{0}\right)$ with $\left(Y_{0}-Y\right) /\left(X_{0}-X\right)(=p / n)$ fixed, the $\mathscr{D}_{0}$ ray expansion matches directly to (168). The matching of the $\mathscr{D}_{0}$ and $(\nu, T)$ scale expansions will show that there are no "gaps" in the asymptotics, which would require the analysis of yet other scales.

We first expand $\mathscr{F}(\nu, T)$ in (170) for $\nu, T \rightarrow \infty$, and we note that, along parabolic scales with $v=O\left(T^{2}\right), v / T^{2}>1$ corresponds to moving from the corner $\left(X_{0}, Y_{0}\right)$ in region $\mathscr{D}_{0}$, while $v / T^{2}<1$ corresponds to moving into $\mathscr{D}_{-}$. By using (190) to approximate the integrand in (170) and shifting the contour to the right, to the range $\operatorname{Re}(\theta) \gg 1$, we are led to

$$
\begin{gathered}
\mathscr{F}(\nu, T) \sim \frac{1}{2 \pi i} \int_{\mathrm{Br}}\left(\frac{\theta}{\nu+\theta}\right)^{1 / 4} e^{\theta T} \\
\cdot \exp \left\{\frac{2}{3}\left[\theta^{3 / 2}-(\theta+\nu)^{3 / 2}\right]\right\} d \theta
\end{gathered}
$$

Here we can view $\theta$ as being scaled to be $O(\nu)$ and $T$ to be $O(\sqrt{v})$. The integrand in (336) has a saddle point where

$$
\begin{aligned}
& \frac{d}{d \theta}\left[\theta T+\frac{2}{3} \theta^{3 / 2}-\frac{2}{3}(\theta+\nu)^{3 / 2}\right]=T+\sqrt{\theta}-\sqrt{\theta+\nu} \\
& \quad=0
\end{aligned}
$$

and this occurs when

$$
\theta=\theta_{s} \equiv \frac{\left(\nu-T^{2}\right)^{2}}{4 T^{2}}=\frac{v^{2}}{4 T^{2}}-\frac{v}{2}+\frac{T^{2}}{4}
$$

Taking $\nu / T^{2}>1$ then a standard saddle point estimate leads to

$$
\begin{aligned}
& \mathscr{F} \sim \frac{1}{\sqrt{2 \pi}}\left[\frac{1}{2 \sqrt{\theta_{s}}}-\frac{1}{2 \sqrt{\theta_{s}+v}}\right]^{-1 / 2}\left(\frac{\theta_{s}}{\theta_{s}+v}\right)^{1 / 4} \\
& \cdot \exp \left\{\theta_{s} T+\frac{2}{3}\left[\theta_{s}^{3 / 2}-\left(\theta_{s}+\nu\right)^{3 / 2}\right]\right\}=\frac{\nu-T^{2}}{2 \sqrt{\pi} T^{3 / 2}} \\
& \cdot \exp \left(\frac{1}{12} T^{3}-\frac{1}{2} \nu T-\frac{1}{4} \frac{\nu^{2}}{T}\right) ; \quad \nu, T \rightarrow \infty .
\end{aligned}
$$

By using (339) in (169) we match the result to the $\mathscr{D}_{0}$ ray expansion, which is $\rho^{-1 / 2} Q(0,0) K(X, Y) e^{\rho \Psi(X, Y)}$, as $(X, Y) \rightarrow$ $\left(X_{0}, Y_{0}\right)$. By separating the exponential factors from the algebraic ones, the matching will hold if both

$$
\begin{aligned}
& \rho^{-1 / 2} K(X, Y) \sim \rho^{-2 / 3} \\
& \cdot \frac{\left(1-\sqrt{\mathrm{X}_{0}}\right)^{5 / 3} X_{0}^{-1 / 6}}{2 \sqrt{\pi}\left(W_{0}+Y_{0}\right)\left(\sqrt{X_{0}}-X_{0}-Y_{0}\right)} \frac{\nu-T^{2}}{T^{3 / 2}},
\end{aligned}
$$




$$
\begin{aligned}
& e^{\rho \Psi(X, Y)} \sim\left(\sqrt{X_{0}}\right)^{n}\left(\frac{Y_{0}}{Y_{0}+W_{0}}\right)^{p} \\
& \cdot \exp \left[-\rho^{1 / 3} \frac{W_{0}}{2 Y_{0}\left(W_{0}+Y_{0}\right)} T_{1}^{2}\right] \\
& \cdot \exp \left[-\frac{W_{0}\left(W_{0}+2 Y_{0}\right)}{6 Y_{0}^{2}\left(W_{0}+Y_{0}\right)^{2}} T_{1}^{3}+\frac{1}{12} T^{3}-\frac{1}{2} \nu T\right. \\
& \left.-\frac{1}{4} \frac{\nu^{2}}{T}\right] .
\end{aligned}
$$

We thus proceed to evaluate $K$ and $\Psi$ as $(X, Y) \rightarrow\left(X_{0}, Y_{0}\right)$ along parabolic scales, where $\left(Y_{0}-Y\right)^{2} /\left(X_{0}-X\right)$ is fixed. In this limit we have $A \rightarrow A_{\text {min }}=1-\sqrt{X_{0}}$ and $B \rightarrow B_{\text {min }}=$ $W_{0} /\left(Y_{0}+W_{0}\right)$, and it is useful to rewrite (68) as

$$
\begin{aligned}
& X_{0}-X=\left(1-e^{-\tau}\right)\left(\frac{X_{0}}{1-A}+A e^{\tau}-1\right), \\
& Y_{0}-Y=\frac{Y_{0}}{1-B}\left(1-e^{-\tau}\right) .
\end{aligned}
$$

The function $K_{0}(A)$ in (275) vanishes in this limit, in view of the factor $(1-A)^{2}-X_{0}$, and we have

$$
\begin{aligned}
& K_{0}(A) \sim \frac{\sqrt{2 Y_{0}}\left(1-\sqrt{X_{0}}\right)\left(A-A_{\min }\right)}{\sqrt{\pi}\left(\sqrt{X_{0}}-Y_{0}-X_{0}\right)\left(W_{0}+Y_{0}\right)}, \\
& A \longrightarrow 1-\sqrt{X_{0}} .
\end{aligned}
$$

Expanding the Jacobian $\Delta$ in (71) for $\tau \rightarrow 0$ and $A \rightarrow A_{\text {min }}$ leads to

$$
\Delta \sim-2 \tau\left(W_{0}+Y_{0}\right) ; \tau \longrightarrow 0, A \longrightarrow 1-\sqrt{X_{0}} .
$$

But from (342) we have $Y_{0}-Y=\rho^{-1 / 3} T_{1} \sim\left(W_{0}+Y_{0}\right) \tau$ so that

$$
|\Delta|^{-1 / 2} \sim \rho^{1 / 6} \frac{1}{\sqrt{2 T}} \frac{\left(1-\sqrt{X_{0}}\right)^{1 / 3}}{X_{0}^{1 / 12} \sqrt{Y_{0}+W_{0}}},
$$

where we wrote the result in terms of $T$ rather than $T_{1}$. From the first expression in (342) we have

$$
X_{0}-X \sim \tau\left[2\left(A-A_{\min }\right)+A_{\min } \tau\right]
$$

so that

$$
\begin{aligned}
A & -A_{\min } \sim \frac{1}{2 \tau}\left[X_{0}-X-\left(1-\sqrt{X_{0}}\right) \tau^{2}\right] \\
& \sim \frac{1}{2} \frac{W_{0}+Y_{0}}{Y_{0}-Y}\left[X_{0}-X-\left(1-\sqrt{X_{0}}\right) \frac{\left(Y_{0}-Y\right)^{2}}{\left(W_{0}+Y\right)^{2}}\right] \\
& =\frac{1}{2} \rho^{-1 / 3} \frac{W_{0}+Y_{0}}{T_{1}}\left[\nu_{1}-\frac{1-\sqrt{X_{0}}}{\left(W_{0}+Y_{0}\right)^{2}} T_{1}^{2}\right] \\
& =\frac{1}{2} \rho^{-1 / 3} \mathrm{X}_{0}^{1 / 6}\left(1-\sqrt{X_{0}}\right)^{1 / 3} \frac{v-T^{2}}{T} .
\end{aligned}
$$

Now

$$
\begin{aligned}
K & (X, Y) \\
& =\left(e^{-\tau}-A\right)^{-1 / 2}\left(e^{-\tau}-B\right)^{-1 / 2}|\Delta|^{-1 / 2} K_{0}(A) \\
& \sim X_{0}^{-1 / 4}\left(Y_{0}+W_{0}\right)^{1 / 2} Y_{0}^{-1 / 2}|\Delta|^{-1 / 2} K_{0}(A),
\end{aligned}
$$

so using (347) and (345) we can easily verify that (340) is satisfied.

To verify (341) we first note that $\Psi\left(X_{0}, Y_{0}\right)=0$,

$$
\begin{aligned}
& \exp \left[\rho\left(X-X_{0}\right) \Psi_{X}\left(X_{0}, Y_{0}\right)\right] \\
& \quad=\exp \left[\rho\left(X_{0}-X\right) \log \left(1-A_{\min }\right)\right]=\left(\sqrt{X_{0}}\right)^{n}, \\
& \exp \left[\rho\left(Y-Y_{0}\right) \Psi_{Y}\left(X_{0}, Y_{0}\right)\right]=\left[\frac{Y_{0}}{\left(Y_{0}+W_{0}\right)}\right]^{p} .
\end{aligned}
$$

Then peeling off the linear part of $\Psi$ near $X=X_{0}, Y=Y_{0}$, (341) is equivalent to showing that

$$
\begin{aligned}
\rho[ & \Psi(X, Y)+\left(X_{0}-X\right) \Psi_{X}\left(X_{0}, Y_{0}\right) \\
+ & \left.\left(Y_{0}-Y\right) \Psi_{Y}\left(X_{0}, Y_{0}\right)\right] \sim-\rho^{1 / 3} \frac{W_{0}}{2 Y_{0}\left(W_{0}+Y_{0}\right)} \\
& \cdot T_{1}^{2}-\frac{W_{0}\left(W_{0}+2 Y_{0}\right)}{6 Y_{0}^{2}\left(W_{0}+Y_{0}\right)^{2}} T_{1}^{3}+\frac{1}{12} T^{3}-\frac{1}{2} \nu T-\frac{1}{4} \frac{\nu^{2}}{T}
\end{aligned}
$$

We can rewrite $\Psi$ in (66) as

$$
\begin{aligned}
& \Psi(X, Y)+\left(X_{0}-X\right) \Psi_{X}\left(X_{0}, Y_{0}\right) \\
& \quad+\left(Y_{0}-Y\right) \Psi_{Y}\left(X_{0}, Y_{0}\right) \\
& =\left(X_{0}-X\right) \log \left(\frac{1-A e^{\tau}}{1-A_{\min }}\right) \\
& \quad+\left(Y_{0}-Y\right) \log \left(\frac{1-B e^{\tau}}{1-B_{\min }}\right) \\
& \quad-X_{0} \log \left(\frac{1-A e^{\tau}}{1-A}\right)-Y_{0} \log \left(\frac{1-B e^{\tau}}{1-B}\right) \\
& \quad+A\left(1-e^{\tau}\right),
\end{aligned}
$$

where we have partially expressed the right-hand side of (351) in terms of $X_{0}-X=\rho^{-2 / 3} v_{1}$ and $Y_{0}-Y=\rho^{-1 / 3} T_{1}$. Next we 
expand (351) as a triple Taylor series about $\tau=0, A=A_{\text {min }}$, and $B=B_{\min }$. We have

$$
\begin{aligned}
A(1 & \left.-e^{\tau}\right)-X_{0} \log \left[1-\frac{A\left(e^{\tau}-1\right)}{1-A}\right] \\
& -Y_{0} \log \left[1-\frac{B\left(e^{\tau}-1\right)}{1-B}\right] \\
= & {\left[\frac{X_{0} A}{1-A}+\frac{Y_{0} B}{1-B}-A\right]\left(e^{\tau}-1\right) } \\
& +\left[\frac{X_{0} A^{2}}{2(1-A)^{2}}+\frac{Y_{0} B^{2}}{2(1-B)^{2}}\right]\left(e^{\tau}-1\right)^{2} \\
& +\left[\frac{X_{0} A^{3}}{3(1-A)^{3}}+\frac{Y_{0} B^{3}}{3(1-B)^{3}}\right]\left(e^{\tau}-1\right)^{3} \\
& +O\left(\tau^{4}\right) .
\end{aligned}
$$

But the relation in (216) implies that the coefficient of $\left(e^{\tau}-1\right)$ in (352) vanishes and also that

$$
B-B_{\min } \sim-\frac{1}{\sqrt{X_{0}}} \frac{Y_{0}}{\left(Y_{0}+W_{0}\right)^{2}}\left(A-A_{\min }\right)^{2} .
$$

By (347), on the $(\nu, T)$ scale, $A-A_{\min }$ is $O\left(\rho^{-1 / 3}\right)$ so that $B-$ $B_{\min }=O\left(\rho^{-2 / 3}\right)$, and thus (352) may be further approximated by (using $\left.\left(e^{\tau}-1\right)^{2}=\tau^{2}+\tau^{3}+O\left(\tau^{4}\right)\right)$

$$
\begin{aligned}
& {\left[\frac{X_{0} A_{\min }^{2}}{2\left(1-A_{\min }\right)^{2}}+\frac{Y_{0} B_{\min }^{2}}{2\left(1-B_{\min }\right)^{2}}+\frac{X_{0} A_{\min }^{3}}{3\left(1-A_{\min }\right)^{3}}\right.} \\
& \left.+\frac{Y_{0} B_{\min }^{3}}{3\left(1-B_{\min }\right)^{3}}\right] \tau^{3}+\left[\frac{X_{0} A_{\min }^{2}}{2\left(1-A_{\min }\right)^{2}}\right. \\
& \left.+\frac{Y_{0} B_{\min }^{2}}{2\left(1-B_{\min }\right)^{2}}\right] \tau^{2}+\left[\frac{X_{0} A_{\min }}{\left(1-A_{\min }\right)^{2}}\right. \\
& \left.+\frac{X_{0} A_{\min }^{2}}{\left(1-A_{\min }\right)^{3}}\right]\left(A-A_{\min }\right) \tau^{2}
\end{aligned}
$$

with an $O\left(\tau^{4}\right)$ error, which is not needed for the matching verification since $\exp \left[O\left(\rho \tau^{4}\right)\right]=\exp \left[O\left(\rho^{-1 / 3}\right)\right] \sim 1$. We also have

$$
\begin{aligned}
& \left(X_{0}-X\right) \log \left(\frac{1-A e^{\tau}}{1-A_{\min }}\right)=\rho^{-2 / 3} v_{1} \log [1 \\
& \quad-\frac{A-A_{\min }}{1-A_{\min }}-\frac{A_{\min } \tau}{1-A_{\min }} \\
& \left.\quad+O\left(\tau^{2}, \tau\left(A-A_{\min }\right)\right)\right] \sim-\frac{\rho^{-2 / 3} v_{1}}{\sqrt{X_{0}}}\left[A-A_{\text {min }}\right.
\end{aligned}
$$

$$
\begin{aligned}
& \left.+A_{\min } \tau\right] \sim-\frac{\rho^{-1} \nu}{X_{0}^{1 / 6}\left(1-\sqrt{X_{0}}\right)^{1 / 3}}\left[\frac{1}{2} X_{0}^{1 / 6}(1\right. \\
& \left.\left.-\sqrt{X_{0}}\right)^{1 / 3} \frac{\nu-T^{2}}{T}+\frac{\left(1-\sqrt{X_{0}}\right)}{W_{0}+Y_{0}} T_{1}\right]=-\frac{v}{2 \rho} \\
& \quad \frac{\nu+T^{2}}{T}, \\
& \left(Y_{0}-Y\right) \log \left(\frac{1-B e^{\tau}}{1-B_{\min }}\right)=\left(Y_{0}-Y\right) \log [1 \\
& \left.-\frac{B-B_{\min }}{1-B_{\min }}-\frac{B_{\min }\left(e^{\tau}-1\right)}{1-B_{\min }}+O\left(\left(B-B_{\min }\right)^{2}\right)\right] \\
& =\rho^{-1 / 3} T_{1}\left[-\frac{B-B_{\min }}{1-B_{\min }}-\frac{B_{\min } \tau}{1-B_{\min }}\right. \\
& \left.-\frac{B_{\min } \tau^{2}}{2\left(1-B_{\min }\right)^{2}}+O\left(\tau^{3}\right)\right] .
\end{aligned}
$$

From (342) we have

$$
\begin{aligned}
\left(Y_{0}-Y\right)(1-B) & =\rho^{-1 / 3} T_{1}\left[1-B_{\min }+O\left(\rho^{-2 / 3}\right)\right] \\
& =Y_{0}\left(1-e^{-\tau}\right) \\
& =Y_{0}\left[\tau-\frac{1}{2} \tau^{2}+O\left(\tau^{3}\right)\right]
\end{aligned}
$$

so we can refine the estimate above (345) to

$$
\tau=\rho^{-1 / 3} \frac{T_{1}}{W_{0}+Y_{0}}+\frac{1}{2} \rho^{-2 / 3} \frac{T_{1}^{2}}{\left(W_{0}+Y_{0}\right)^{2}}+O\left(\rho^{-1}\right) .
$$

Adding the expressions in (354) and (355) and using the estimates in (347), (353), and (357), (351) becomes, when multiplied by $\rho$,

$$
\begin{aligned}
& {\left[\frac{\left(1-\sqrt{X_{0}}\right)^{2}}{2}+\frac{W_{0}^{2}}{2 Y_{0}}+\frac{\left(1-\sqrt{X_{0}}\right)^{3}}{3 \sqrt{X_{0}}}+\frac{W_{0}^{3}}{3 Y_{0}^{2}}\right]} \\
& \cdot \frac{T_{1}^{3}}{\left(W_{0}+Y_{0}\right)^{3}}+\left[1-\sqrt{X_{0}}+\frac{\left(1-\sqrt{X_{0}}\right)^{2}}{\sqrt{X_{0}}}\right] \\
& +\frac{X_{0}^{1 / 6}\left(1-\sqrt{X_{0}}\right)^{1 / 3}}{2\left(W_{0}+Y_{0}\right)^{2}} \frac{T_{1}^{2}\left(\nu-T^{2}\right)}{T} \\
& +\left[\frac{\left(1-\sqrt{X_{0}}\right)^{2}}{2}+\frac{W_{0}^{2}}{2 Y_{0}}\right] \\
& +\left[\rho^{1 / 3} \frac{T_{1}^{2}}{\left(W_{0}+Y_{0}\right)^{2}}+\frac{T_{1}^{3}}{\left(W_{0}+Y_{0}\right)^{3}}\right]-\frac{\nu^{2}}{2 T}-\frac{\nu T}{2} \\
& +\frac{W_{0}+Y_{0}}{Y_{0}} T_{1} \frac{Y_{0}}{\left(W_{0}+Y_{0}\right)^{2}} \frac{1}{\sqrt{X_{0}}}
\end{aligned}
$$




$$
\begin{aligned}
& \cdot \frac{X_{0}^{1 / 3}\left(1-\sqrt{X_{0}}\right)^{2 / 3}}{4}\left(\frac{\nu}{T}-T\right)^{2}-\frac{W_{0}\left(W_{0}+Y_{0}\right)}{w Y_{0}^{2}} \\
& \cdot T_{1}\left(\frac{T_{1}}{W_{0}+Y_{0}}\right)^{2} \\
& -\frac{W_{0}}{Y_{0}}\left[\rho^{1 / 3} \frac{T_{1}^{2}}{W_{0}+Y_{0}}+\frac{T_{1}^{3}}{2\left(W_{0}+Y_{0}\right)^{2}}\right] .
\end{aligned}
$$

Comparing (358) to (350) we find, after some simplification, that they are identical. The comparison is facilitated by separately comparing terms proportional to $\rho^{1 / 3} T_{1}^{2}, \nu^{2} / T$, $\nu T$, and $T^{3}$. This verifies the matching between the $\mathscr{D}_{0}$ ray expansion and that on the $(\nu, T)$ scale. The Airy functions that arose on the $(\nu, T)$ scale disappear in the limit where $\nu, T \rightarrow \infty$. If we let $v, T \rightarrow \infty$ with $v / T^{2}<1$, then the expansion of $\mathscr{F}(\nu, T)$ is much different, and now we have

$$
\begin{aligned}
& \mathscr{F}(\nu, T) \\
& \quad \sim \frac{1}{2 \sqrt{\pi} \mathrm{Ai}^{\prime}\left(r_{0}\right)} \frac{1}{v^{1 / 4}} \exp \left[-\frac{2}{3} \nu^{3 / 2}+r_{0}(T-\sqrt{\nu})\right],
\end{aligned}
$$

as the pole at $\theta=r_{0}$ determines the asymptotic behavior. We can show that then (169) with (359) agrees with the ray expansion in $\mathscr{D}_{-}$, that is, $C_{1} L(X, Y) e^{\rho \Phi(X, Y)} e^{\rho^{1 / 3} \Phi_{*}\left(s_{1}\right)}$, as the latter is expanded for $(X, Y) \rightarrow\left(X_{0}, Y_{0}\right)$, for parameter region $\mathscr{R}_{4}$. The case where $\nu / T^{2} \approx 1$ will be important in determining the transition layer expansion that applies where $\mathscr{D}_{0}$ meets $\mathscr{D}_{-}$, which is along the curve $Y=Y_{c}(X)$. Then neither (339) nor (359) apply.

\section{Asymptotic Expansions near State Space Boundaries}

We discuss the four boundary segments, $X=0, X=X_{0}$, $Y=0$, and $Y=Y_{0}$, of the state space rectangle, avoiding for now the corner points. From Figures $3-6$ we see that $\mathscr{D}_{+}$will border $X=0$ and $X=X_{0}$ for parameter regions $\mathscr{R}_{2}$ and $\mathscr{R}_{3}$ and also border $Y=0$ for region $\mathscr{R}_{1}$. As we previously discussed the ray expansion in $\mathscr{D}_{+}$remains valid near $X=X_{0}$ but breaks down near $Y=0\left(\mathscr{R}_{1}\right)$ and $X=0\left(\mathscr{R}_{2} \cup \mathscr{R}_{3}\right)$. The analysis of $Y=0$ (corresponding to the scales $r=O(1)$ and $r=O(\sqrt{\rho}))$ is identical to that of the infinite capacity model, and we show in [10] how to construct appropriate boundary layer corrections; this leads to (88)-(95) in Proposition 8. The analysis near $X=0$ (with $0<Y<\left[\left(X_{0}+Y_{0}\right)^{2}-X_{0}\right] /\left(X_{0}+\right.$ $\left.\left.Y_{0}\right)^{2}\right)$ is also very similar to that in [10], except that if $X_{0}+$ $Y_{0}<1\left(\mathscr{R}_{2} \cup \mathscr{R}_{3}\right)$ we must use the values of $C(\rho)$ in (32) and (33). Thus both the $\mathscr{D}_{+}$ray expansion and the boundary layer expansion, which applies for $k=O(1)$, are rescaled by a constant. We thus obtain (114).

For parameter region(s) $\mathscr{R}_{2} \cup \mathscr{R}_{3} \cup \mathscr{R}_{4}$, $\mathscr{D}_{-}$meets the line $X=X_{0}$, and there the expansions in (109)-(112) apply. Their derivation for $\mathscr{R}_{2}$ is identical to that in [10], while for the other subcases we must simply multiply both the $\mathscr{D}_{\text {_ }}$ ray expansion and the boundary layer corrections by the appropriate $C_{1}(\rho)$, from among (35), (36), (37), and (41). Similarly, the boundary layer correction along $Y=0$ is given by (96), with the appropriate value of $C_{1}(\rho)$. For $\mathscr{R}_{2} \cup \mathscr{R}_{3}$ this covers the entire range $0<X<X_{0}$, but for $\mathscr{R}_{4}$ only the range $X_{L}<X<X_{0}$ (with $X_{L}$ defined in item (vii) in Proposition 8), as in the range $0<X<X_{L}, \mathscr{D}_{0}$ meets $Y=0$.

Here we will only examine where $\mathscr{D}_{0}$ meets the state space boundaries, and this occurs along $Y=Y_{0}$ for all regions $\mathscr{R}_{j}$, along $X=0$ with $\left[\left(X_{0}+Y_{0}\right)^{2}-X_{0}\right] /\left(X_{0}+Y_{0}\right)^{2}<Y<Y_{0}$ for $\mathscr{R}_{1} \cup \mathscr{R}_{2} \cup \mathscr{R}_{3}$, and all $0<Y<Y_{0}$ for $\mathscr{R}_{4}$, and along $Y=0$ with $0<X<X_{L}$ for $\mathscr{R}_{4}$ only. We thus proceed to construct boundary layer corrections to the $\mathscr{D}_{0}$ ray expansion for these three boundary segments.

5.1. The Boundary Segment $X=0$. We consider the scale $k=$ $O(1)$ and let

$$
\pi(k, r)=C_{2}(\rho) e^{\rho \Psi(0, Y)} \rho^{k} \Pi_{k}(Y ; \rho) .
$$

Then the main balance equation (3), after dividing by $\exp [\rho \Psi(0, Y)]$, becomes

$$
\begin{aligned}
& {[\rho(1+Y)+k] \Pi_{k}(Y ; \rho)=(\rho Y+1) \Pi_{k}\left(Y+\rho^{-1} ; \rho\right)} \\
& \cdot \exp \left\{\rho\left[\Psi\left(0, Y+\rho^{-1}\right)-\Psi(0, Y)\right]\right\}+\rho(k+1) \\
& \cdot \Pi_{k+1}(Y ; \rho)+\Pi_{k-1}(Y ; \rho) .
\end{aligned}
$$

Taking $\Pi_{k}(Y)$ to be the leading order approximation to $\Pi_{k}(Y ; \rho)$, dividing (361) by $\rho$, and letting $\rho \rightarrow \infty$ lead to the limiting equation

$$
\left[1+Y-Y e^{\Psi_{Y}(0, Y)}\right] \Pi_{k}(Y)=(k+1) \Pi_{k+1}(Y),
$$

$k \geqslant 1$.

By examining the boundary condition in (6) we conclude that (362) holds also if $k=0$, and thus

$$
\Pi_{k}(Y)=\frac{\Pi_{0}(Y)}{k !}\left[1+Y-Y e^{\Psi_{Y}(0, Y)}\right]^{k}
$$

To determine $C_{2}(\rho)$ and $\Pi_{0}(Y)$ we asymptotically match (360) to the $\mathscr{D}_{0}$ ray expansion. We thus expand $\rho^{-1 / 2} Q(0,0) K(X, Y) e^{\rho \Psi(X, Y)}$ as $X \rightarrow 0$ and compare the result to the large $k$ expansion of (360). By using Stirling's formula we see that the matching is possible if, as $X \rightarrow 0$,

$$
\begin{aligned}
& \Psi(X, Y) \\
& =\Psi(0, Y)-X \log X \\
& \quad+X\left[1+\log \left(1+Y-Y e^{\Psi_{Y}(0, Y)}\right)\right]+o(X), \\
& \frac{Q(0,0)}{\sqrt{\rho}} K(X, Y) \sim \frac{C_{2}(\rho)}{\sqrt{2 \pi \rho X}} \Pi_{0}(Y), \quad X \longrightarrow 0 .
\end{aligned}
$$

Note that the exponential factor in (360) must be included for the matching to be possible. Thus we could set $C_{2}(\rho)=$ $Q(0,0)$ and then $\Pi_{0}(Y)$ will be the limit of $\sqrt{2 \pi X} K(X, Y)$ as $X \rightarrow 0$, which we show below to be finite and nonzero. 
From (68) we see that $X=0$ corresponds to $A=e^{-\tau}$ and we set $A_{1}(Y)=A(0, Y)$, where we view $A$ as a function of $(X, Y)$ via the mapping in (68). Then we also set $B_{1}(Y)=$ $B(0, Y)$ and from (68) find that

$$
A_{1}-B_{1}=\frac{Y\left(1-A_{1}\right)}{Y_{0}-Y} \text {. }
$$

Using (366) in (67) leads to a quadratic equation for $A_{1}(Y)$, whose solution is given by (118). Also, replacing $\left(e^{\tau}, A, B\right)$ by $\left(1 / A_{1}, A_{1}, B_{1}\right)$ in (66) leads to the expression in (119) for $\Psi(0, Y)$, which is an explicit function of $Y$. As $X \rightarrow 0$, $X /\left(e^{-\tau}-A\right)$ is finite and from (68) we obtain

$$
e^{-\tau}-A \sim \frac{A_{1}\left(1-A_{1}\right)}{A_{1}\left(A_{1}+X_{0}-2\right)+1} X, \quad X \longrightarrow 0 .
$$

Evaluating the Jacobian $\Delta$ in (71) along $X=0$ leads to $\Delta_{L}(Y)$ in (120). Thus as $X \rightarrow 0$ we have

$$
\begin{gathered}
\left(e^{-\tau}-A\right)^{-1 / 2}\left(e^{-\tau}-B\right)^{-1 / 2}|\Delta|^{-1 / 2} K_{0}(A) \\
\sim\left(A_{1}-B_{1}\right)^{-1 / 2}\left|\Delta_{L}\right|^{-1 / 2} K_{0}\left(A_{1}\right) \\
\cdot \sqrt{\frac{A_{1}\left(A_{1}+X_{0}-2\right)+1}{A_{1}\left(1-A_{1}\right)}} \frac{1}{\sqrt{X}} .
\end{gathered}
$$

We have thus identified $\Pi_{0}(Y)$ as

$$
\begin{aligned}
\Pi_{0}(Y)= & \left(A_{1}-B_{1}\right)^{-1 / 2}\left|\Delta_{L}\right|^{-1 / 2} \sqrt{2 \pi} K_{0}\left(A_{1}\right) \\
& \cdot \sqrt{\frac{A_{1}\left(A_{1}+X_{0}-2\right)+1}{A_{1}\left(1-A_{1}\right)}} .
\end{aligned}
$$

Using (366) and $K_{0}(\cdot)$ in (275) we see that (360) agrees with (117) as $X \rightarrow 0$, if

$$
1+Y-Y e^{\Psi_{Y}(0, Y)}=\frac{A_{1}\left(A_{1}+X_{0}-2\right)+1}{1-A_{1}}
$$

and $\Psi$ has the small $X$ behavior indicated in (364). Now, $\Psi_{Y}=$ $-\log \left(1-B e^{\tau}\right)$ so that $\Psi_{Y}(0, Y)=-\log \left(1-B_{1} / A_{1}\right)$ and using (366) leads to

$$
\begin{aligned}
1+Y-Y e^{\Psi_{Y}(0, Y)} & =1+Y-\frac{Y A_{1}}{A_{1}-B_{1}} \\
& =1+Y-\frac{\left(Y_{0}-Y\right)}{1-A_{1}} A_{1} .
\end{aligned}
$$

But from (118) we see that $A_{1}^{2}+\left(X_{0}+Y_{0}-1\right) A_{1}-Y=0$ so that the right-hand sides of (370) and (371) agree.

Using (68) we have, as $X \rightarrow 0$,

$$
\begin{aligned}
& \Psi_{X}+\log X=-\log \left(1-A e^{\tau}\right) \\
&+\log \left[\left(e^{-\tau}-A\right)\left(\frac{X_{0}}{1-A}-1+e^{\tau}\right)\right] \\
& \sim \log \left[\left(\frac{X_{0}}{1-A}-1\right) e^{-\tau}+1\right] \\
& \sim \log \left[\frac{X_{0} A_{1}}{1-A_{1}}+1-A_{1}\right] .
\end{aligned}
$$

Integrating the above, noting that $\Psi_{X}+\log X$ is an analytic function of $X$, we conclude that

$$
\begin{aligned}
& \Psi(X, Y)+X \log X-X \\
& \quad=X \log \left(\frac{A_{1}^{2}+\left(X_{0}-2\right) A_{1}+1}{1-A_{1}}\right) O\left(X^{2}\right)
\end{aligned}
$$

and this verifies (364). We have thus shown that the asymptotic matching holds and also determined $\Pi_{0}(Y)$. This completes the derivation of (117)-(120).

For region $\mathscr{R}_{4}$, (117) holds for all $0<Y<Y_{0}$, and then the expansion matches to the corner expansions that apply for $Y=O\left(\rho^{-1}\right)(r=O(1))$ and $Y=Y_{0}-O\left(\rho^{-1}\right)(p=R-r=$ $O(1))$. But for region $\mathscr{R}_{1} \cup \mathscr{R}_{2} \cup \mathscr{R}_{3}$ and $k=O(1)$, (117) holds only for $Y_{U}<Y<Y_{0}$, while (114) applies for $0<Y<Y_{U}$. To connect these it is necessary to construct another expansion for $Y-Y_{U}=O\left(\rho^{-1 / 2}\right)$. Using the variables $k$ and $y_{*} \equiv \sqrt{\rho}(Y-$ $\left.Y_{U}\right)=O(1)$ we conclude from (3) and (6) that

$$
\begin{aligned}
\pi(k, r) \sim & C_{3}(\rho) e^{\rho \Psi\left(0, Y_{U}+\rho^{-1 / 2} y_{*}\right)} \\
& \cdot \frac{\rho^{k}}{k !}\left(1+Y_{0}-\frac{Y_{0}}{X_{0}+Y_{0}}\right)^{k} \Pi_{*}\left(y_{*}\right),
\end{aligned}
$$

where we note that $1+Y-Y e^{\Psi_{Y}(0, Y)}=1+Y_{0}-Y_{0} /\left(X_{0}+Y_{0}\right)$ when $Y=Y_{U}$, since $A_{1}\left(Y_{U}\right)=Y_{0} /\left(X_{0}+Y_{0}\right)$. To determine $\Pi_{*}(\cdot)$ we first infer its behaviors as $y_{*} \rightarrow \pm \infty$ by asymptotically matching (374) to the expansions that apply for $Y<Y_{U}$ and $Y>Y_{U}$.

We expand the result in (114) as $Y \uparrow Y_{U}$. We have $D_{0}\left(Y_{U}\right)=D_{0}(\tilde{Y}(0))=Y_{0} /\left(X_{0}+Y_{0}\right)$ so that

$$
\begin{aligned}
1+ & Y_{U}-D_{0}^{2}\left(Y_{U}\right) \\
& =\frac{A_{1}\left(Y_{U}\right)\left(A_{1}\left(Y_{U}\right)+X_{0}-2\right)+1}{1-A_{1}\left(Y_{U}\right)} \\
& =Y_{0}+\frac{X_{0}}{X_{0}+Y_{0}}, \\
\Psi_{+, Y} & \left(0, Y_{U}\right)=\Psi_{Y}\left(0, Y_{U}\right) \\
& =-\log \left[\frac{\left(X_{0}+Y_{0}\right)^{2}-X_{0}}{Y_{0}}\right] .
\end{aligned}
$$

After some calculation we find that

$$
\begin{aligned}
& \frac{1-D_{0}\left(Y_{U}\right)}{D_{0}\left(Y_{U}\right)} \\
& \cdot \frac{Y_{U}}{\sqrt{X_{0}}}\left[Y_{U}+2 D_{0}^{2}\left(Y_{U}\right)\left(1-D_{0}\left(Y_{U}\right)\right)\right]^{-1 / 2} \\
& =\sqrt{\frac{X_{0}}{Y_{0}}}\left[1-\frac{X_{0}}{\left(X_{0}+Y_{0}\right)^{2}}\right] \\
& \cdot \frac{\left(X_{0}+Y_{0}\right)^{3 / 2}}{\sqrt{\left(X_{0}+Y_{0}\right)^{3}+X_{0}\left(Y_{0}-X_{0}\right)}} .
\end{aligned}
$$


Furthermore,

$$
\begin{aligned}
\Psi\left(0, Y_{U}\right)= & \Psi_{+}\left(0, Y_{U}\right)+1-X_{0}-X_{0} \\
& +\left(X_{0}+Y_{0}\right) \log \left(X_{0}+Y_{0}\right)
\end{aligned}
$$

and we note that, in view of Propositions 1 and 2,

$$
C(\rho) \sim \sqrt{2 \pi} \sqrt{X_{0}+Y_{0}} Q(0,0) e^{\rho\left[\Psi\left(0, Y_{U}\right)-\Psi_{+}\left(0, Y_{U}\right)\right]}
$$

which holds in regions $\mathscr{R}_{1} \cup \mathscr{R}_{2} \cup \mathscr{R}_{3}$. Using (375)-(378) to infer the behavior of (114) as $Y \uparrow Y_{U}$ and comparing the result to (374) we can take $C_{3}(\rho)=\sqrt{2 \pi \rho} Q(0,0)$ and then, as $y_{*} \rightarrow-\infty$,

$$
\begin{aligned}
\Pi_{*}\left(y_{*}\right) \exp \left[\frac{1}{2} \Psi_{Y Y}\left(0, Y_{U}\right) y_{*}^{2}\right] \\
\sim \frac{\left(X_{0}+Y_{0}\right)^{2}-X_{0}}{\sqrt{\left(X_{0}+Y_{0}\right)^{3}+X_{0}\left(Y_{0}-X_{0}\right)}} \sqrt{\frac{X_{0}}{Y_{0}}} \\
\cdot \exp \left[\frac{1}{2} \Psi_{+, Y Y}\left(0, Y_{U}\right) y_{*}^{2}\right] .
\end{aligned}
$$

Next we expand (117) as $Y \downarrow Y_{U}$. Since $\left(X_{0}+Y_{0}\right) A_{1}\left(Y_{U}\right)=$ $Y_{0}$, (117) is singular in this limit, and we use

$$
\begin{aligned}
Y_{0} & -A_{1}(Y)\left(X_{0}+Y_{0}\right) \\
& \sim-A_{1}^{\prime}\left(Y_{U}\right)\left(X_{0}+Y_{0}\right)\left(Y-Y_{U}\right) \\
& =-A_{1}^{\prime}\left(Y_{U}\right)\left(X_{0}+Y_{0}\right) y_{*} \rho^{-1 / 2}
\end{aligned}
$$

to obtain

$$
\begin{aligned}
& \sqrt{\frac{Y_{0}}{Y\left(Y_{0}-Y\right)} \frac{\sqrt{A_{1}\left(A_{1}+X_{0}-2\right)+1}\left[\left(1-A_{1}\right)^{2}-X_{0}\right] \sqrt{A_{1}}}{\left|\Delta_{L}(Y)\right|^{1 / 2}\left[Y_{0}-A_{1}\left(X_{0}+Y_{0}\right)\right]}} \\
& \sim \frac{\sqrt{\rho}}{y_{*}} \frac{\sqrt{A_{1}\left(Y_{U}\right)\left(A_{1}\left(Y_{U}\right)+X_{0}-2\right)+1}\left[\left(1-A_{1}\left(Y_{U}\right)\right)^{2}-X_{0}\right] \sqrt{A_{1}\left(Y_{U}\right)}}{\left|\Delta_{L}\left(Y_{U}\right)\right|^{1 / 2}\left[-\left(X_{0}+Y_{0}\right)\right] A_{1}^{\prime}\left(Y_{U}\right)} \\
& =\frac{\sqrt{\rho}}{y_{*}} \frac{X_{0}}{\left(X_{0}+Y_{0}\right)^{5 / 2}} \sqrt{\left(X_{0}+Y_{0}\right)^{2}-X_{0}} \sqrt{\left(X_{0}+Y_{0}\right)^{2}+Y_{0}-X_{0}} \\
& =\frac{\sqrt{\rho}}{y_{*}} \sqrt{\frac{X_{0}}{Y_{0}}}\left[\left(X_{0}+Y_{0}\right)^{2}-X_{0}\right]\left[\left(X_{0}+Y_{0}\right)^{3}+X_{0}\left(Y_{0}-X_{0}\right)\right]^{-1 / 2} \frac{1}{a_{c}},
\end{aligned}
$$

where $a_{c}$ was defined in (125). To obtain (381) we also used

$$
\begin{aligned}
& A_{1}^{\prime}\left(Y_{U}\right)=\frac{X_{0}+Y_{0}}{\left(X_{0}+Y_{0}\right)^{2}+Y_{0}-X_{0}}, \\
& \left|\Delta_{L}\left(Y_{U}\right)\right| \\
& \quad=\frac{\left(X_{0}+Y_{0}\right)^{2}+Y_{0}-X_{0}}{\left(X_{0}+Y_{0}\right)^{2}}\left[\left(X_{0}+Y_{0}\right) Y_{0}+X_{0}\right],
\end{aligned}
$$

which follow from (118) and (120). Denoting the ratio of the left- and right-hand sides of (379) by $f\left(y_{*}\right)$, the matching conditions as $y_{*} \rightarrow \pm \infty$ may be written as

$$
\begin{aligned}
& f\left(y_{*}\right) \longrightarrow 1, \quad y_{*} \longrightarrow-\infty \\
& f\left(y_{*}\right) \sim \frac{1}{\sqrt{2 \pi} a_{c} y_{*}} \\
& \quad \cdot \exp \left\{\frac{y_{*}^{2}}{2}\left[\Psi_{+, Y Y}\left(0, Y_{U}\right)-\Psi_{Y Y}\left(0, Y_{U}\right)\right]\right\},
\end{aligned}
$$

After some calculation we can show that

$$
\Psi_{+, Y Y}\left(0, Y_{U}\right)-a_{c}^{2}=\Psi_{Y Y}\left(0, Y_{U}\right)
$$

so that the error function

$$
f\left(y_{*}\right)=\frac{1}{\sqrt{2 \pi}} \int_{a_{c} y_{*}}^{\infty} e^{-u^{2} / 2} d u
$$

satisfies the conditions in (383). To determine $f\left(y_{*}\right)$ completely we need to match (374), with $C_{3}=\sqrt{2 \pi \rho} Q(0,0)$, to the expansion in the transition layer where $\mathscr{D}_{+}$and $\mathscr{D}_{0}$ meet, which is given by (176). This matching has $X \rightarrow 0$ in (176) and $k \rightarrow \infty$ in (374), with $\eta_{*}$ fixed. Noting that as $X \rightarrow 0$

$$
\begin{aligned}
\eta_{*} & =\sqrt{\rho}[Y-\tilde{Y}(X)]=\sqrt{\rho}\left[Y-Y_{U}+O(X)\right] \\
& =y_{*}+O\left(\rho^{-1 / 2}\right)
\end{aligned}
$$

we can show, using (178) and (184)-(187), that $b\left(t_{*}\right) \rightarrow a_{c}^{-2}$ as $X \rightarrow 0$ and thus (385) determines $f\left(y_{*}\right)$ for all $y_{*}=O(1)$.

5.2. The Boundary Segment $Y=0$. We consider $r=O(1)$, in ranges where $\mathscr{D}_{0}$ meets the $x$-axis, which can occur only for 
$\mathscr{R}_{4}$. The analysis for where $\mathscr{D}_{+}\left(\right.$region $\mathscr{R}_{1}$ ) or $\mathscr{D}_{-}$(regions $\mathscr{R}_{2}$ and $\mathscr{R}_{3}$ ) meet the $x$-axis is very similar to that in [10] so we omit it. By examining (3), noting that this equation holds even if $r=0$, on the scale $r=O(1)$ with $k=\rho X, X>0$, we find that

$$
\pi(k, r) \sim C_{4}(\rho) \rho^{r} \widetilde{\Pi}_{r}(X) e^{\rho \Psi(X, 0)},
$$

where $\widetilde{\Pi}_{r}(X)$ will satisfy

$$
\begin{aligned}
& {\left[1+X-e^{-\Psi_{X}(X, 0)}-X e^{\Psi_{X}(X, 0)}\right] \widetilde{\Pi}_{r}(X)} \\
& =(r+1) \widetilde{\Pi}_{r+1}(X) .
\end{aligned}
$$

Noting that $\Psi_{X}(X, 0)=-\log \left(1-A e^{\tau}\right)$, we define $A_{0}(\cdot)$ by $A_{0}(X)=A(X, 0)$. A ray in $\mathscr{D}_{0}$ reaches the $x$-axis when $e^{-\tau}=$ $B$ so we also let $B_{0}(X)=B(X, 0)$. Then evaluating (197) along $Y=0$ leads to

$$
\begin{aligned}
1+ & X-X e^{\Psi_{X}(X, 0)}-e^{-\Psi_{X}(X, 0)}=\lim _{Y \rightarrow 0}\left[Y e^{\Psi_{Y}}\right] \\
& =\lim _{e^{-\tau} \rightarrow B}\left\{Y_{0} \frac{B-e^{-\tau}}{B-1} \exp \left[-\log \left(1-B e^{\tau}\right)\right]\right\} \\
& =\frac{Y_{0} B_{0}}{1-B_{0}}
\end{aligned}
$$

so that the solution to $(388)$ is

$$
\widetilde{\Pi}_{r}(X)=\widetilde{\Pi}_{0}(X) \frac{1}{r !}\left[\frac{Y_{0} B_{0}(Y)}{1-B_{0}(Y)}\right]^{r} .
$$

We proceed to determine the constant $C_{4}(\rho)$ and function $\widetilde{\Pi}_{0}(\cdot)$ by asymptotically matching $(387)$ to the $\mathscr{D}_{0}$ ray expansion.

First we note that setting $e^{-\tau}=B_{0}$ in (66) and replacing $(A, B)$ by $\left(A_{0}, B_{0}\right)$ lead to the expression in (99) for $\Psi(X, 0)$. As $Y \rightarrow 0$ we also have, in view of (389),

$$
\Psi_{Y}(X, Y)=-\log Y+\log \left(\frac{Y_{0} B_{0}}{1-B_{0}}\right)+o(1) .
$$

By integrating (391) we see that the $\mathscr{D}_{0}$ ray expansion behaves for $Y \rightarrow 0$ as

$$
\begin{gathered}
\frac{Q(0,0)}{\sqrt{\rho}} K(X, Y) e^{-\rho Y \log Y} e^{\rho Y} \exp \left[\rho Y \log \left(\frac{Y_{0} B_{0}}{1-B_{0}}\right)\right] \\
=\frac{\rho^{r}}{r^{r}} e^{r}\left(\frac{Y_{0} B_{0}}{1-B_{0}}\right)^{r} \frac{\left(1-X_{0}-Y_{0}\right)}{\sqrt{\rho}} K(X, Y) .
\end{gathered}
$$

Here we used $Q(0,0) \sim 1-X_{0}-Y_{0}$ (in $\left.\mathscr{R}_{4}\right)$ and $\rho Y=$ $r$. Expanding $r$ ! in (390) by Stirling's formula we see the matching is possible if $C_{4}=1-X_{0}-Y_{0}$ and

$$
K(X, Y) \sim \frac{1}{\sqrt{2 \pi Y}} \widetilde{\Pi}_{0}(X), \quad Y \longrightarrow 0 .
$$

We denote by $g(X)$ the limit of $[\sqrt{2 \pi Y} K(X, Y)]$ as $Y \rightarrow 0$. Writing

$$
\begin{aligned}
& K(X, Y) \\
& \quad=\left(e^{-\tau}-B\right)^{-1 / 2}\left(e^{-\tau}-A\right)^{-1 / 2}|\Delta|^{-1 / 2} K_{0}(A)
\end{aligned}
$$

we simply replace $A$ by $A_{0}(X)$ in the factors that are not singular and use

$$
\left(e^{-\tau}-B\right)^{-1 / 2}=\left[\frac{Y}{Y_{0}}(1-B)\right]^{-1 / 2} \sim \frac{1}{\sqrt{Y}} \sqrt{\frac{Y_{0}}{1-B_{0}}}
$$

Then $g(X)$ becomes precisely the expression in (102). We have thus determined $\widetilde{\Pi}_{0}(X)$ as $g(X)$ and thus derived (98)-(102).

The approximation in (387) applies only for $0<X<X_{L}$, and (96) applies for $X_{L}<X<X_{0}$. But for $X \approx X_{L}$ a new approximation is needed, as $g(X)$ vanishes as $X \uparrow X_{L}$, due to the factor $\left(1-A_{0}\right)^{2}-X_{0}$, since $1-A_{0}\left(X_{L}\right)=\sqrt{X_{0}}$. Since $B_{0}\left(X_{L}\right)=W_{0} /\left(W_{0}+Y_{0}\right)$ we find from (102) that

$$
g(X)
$$

$$
\begin{array}{r}
\sim \sqrt{\frac{W_{0}+Y_{0}}{2 W_{0}+Y_{0}} \frac{\sqrt{1-\sqrt{X_{0}}}}{\sqrt{X_{0}}} \frac{X_{0}-\left(1-A_{0}\right)^{2}}{\left(\sqrt{X_{0}}-X_{0}-Y_{0}\right)^{3 / 2}},} \\
X \longrightarrow X_{L} .
\end{array}
$$

By implicit differentiation of (100) we find that

$$
A_{0}^{\prime}\left(X_{L}\right)=-\frac{W_{0}\left(W_{0}+Y_{0}\right)}{Y_{0}\left(2 W_{0}+Y_{0}\right)}
$$

Using

$$
\begin{aligned}
X_{0}-\left(1-A_{0}\right)^{2} & \sim 2\left(1-A_{0}\right) A_{0}^{\prime}\left(X_{L}\right)\left(X-X_{L}\right) \\
& =2 \sqrt{X_{0}} A_{0}^{\prime}\left(X_{L}\right)\left(X-X_{L}\right)
\end{aligned}
$$

and (397) we obtain from (396) the expression in (104), which will be used for asymptotic matching verifications.

We consider the scale $X-X_{L}=\rho^{-1 / 3} \Lambda=O\left(\rho^{-1 / 3}\right)$, retaining $r=O(1)$. From the balance equations we can conclude that the expansion has the form

$$
\begin{aligned}
& C_{5}(\rho) e^{\rho \Psi(X, 0)}\left[\frac{Y_{0} B_{0}\left(X_{L}\right)}{1-B_{0}\left(X_{L}\right)}\right]^{r} \frac{\rho^{r}}{r !} g\left(X_{L}\right) \sim C_{5}(\rho) \\
& \cdot e^{\rho \Psi\left(X_{L}, 0\right)} \frac{\rho^{r} W_{0}^{r}}{r !} \\
& \cdot \exp \left[\rho^{2 / 3} \Psi_{X}\left(X_{L}, 0\right) \Lambda+\frac{1}{2} \rho^{1 / 3} \Psi_{X X}\left(X_{L}, 0\right) \Lambda^{2}\right] \\
& \cdot \widehat{\Pi}(\Lambda),
\end{aligned}
$$

where the last exponential factor follows from expanding $\Psi(X, 0)$ about $X=X_{L}$, and these are necessary to have a chance of matching to the expansion for $X<X_{L}$. By comparing (399) to the behavior of (98) as $X \uparrow X_{L}$ we immediately conclude that $C_{5}(\rho)=O\left(\rho^{-1 / 3}\right)$ so we set $C_{5}(\rho)=\rho^{-1 / 3}\left(1-X_{0}-Y_{0}\right)$, and then

$$
\widehat{\Pi}(\Lambda) \sim \exp \left[\frac{1}{6} \Psi_{X X X}\left(X_{L}, 0\right) \Lambda^{3}\right]
$$




$$
\cdot \frac{2\left(W_{0}+Y_{0}\right)^{3 / 2} W_{0}^{5 / 4}}{Y_{0}\left(2 W_{0}+Y_{0}\right)^{3 / 2}\left(\sqrt{X_{0}}-X_{0}-Y_{0}\right)^{3 / 2}}(-\Lambda),
$$$$
\Lambda \longrightarrow-\infty \text {. }
$$

(400)

From (101) we find that

$$
\frac{Y_{0}}{\left(1-B_{0}\right)^{2}} B_{0}^{\prime}(X)=\left[1-\frac{X_{0}}{\left(1-A_{0}\right)^{2}}\right] A_{0}^{\prime}(X)
$$

so that $B_{0}^{\prime}\left(X_{L}\right)=0$. Then from (99) we find that

$$
\Psi_{X X}(X, 0)=\left(\frac{1}{B_{0}}-\frac{1}{B_{0}-A_{0}}\right) B_{0}^{\prime}+\frac{A_{0}^{\prime}}{B_{0}-A_{0}}
$$

and thus $\Psi_{X X}\left(X_{L}, 0\right)=A_{0}^{\prime}\left(X_{L}\right) /\left[B_{0}\left(X_{L}\right)-1+\sqrt{X_{0}}\right]$ is as in (107). From (402) we also conclude that

$$
\begin{aligned}
& \Psi_{X X X}\left(X_{L}, 0\right) \\
& =\left[\frac{1}{B_{0}\left(X_{L}\right)}-\frac{1}{B_{0}\left(X_{L}\right)-A_{0}\left(X_{L}\right)}\right] B_{0}^{\prime \prime}\left(X_{L}\right) \\
& \quad+\frac{A_{0}^{\prime \prime}\left(X_{L}\right)}{B_{0}\left(X_{L}\right)-A_{0}\left(X_{L}\right)} \\
& \quad+\left[\frac{A_{0}^{\prime}\left(X_{L}\right)}{B_{0}\left(X_{L}\right)-A_{0}\left(X_{L}\right)}\right]^{2} .
\end{aligned}
$$

and thus

$$
\begin{aligned}
\Psi_{X X X}\left(X_{L}, 0\right) & \\
=- & \frac{2}{\sqrt{X_{0}}} \frac{W_{0}^{5 / 2}\left(W_{0}+Y_{0}\right)^{3}}{Y_{0}^{2}\left(2 W_{0}+Y_{0}\right)^{3}} \frac{1}{\sqrt{X_{0}}-X_{0}-Y_{0}} \\
& +\frac{W_{0}\left(W_{0}+Y_{0}\right)^{4}}{Y_{0}^{2}\left(2 W_{0}+Y_{0}\right)^{2}} \frac{1}{\left(\sqrt{X_{0}}-X_{0}-Y_{0}\right)^{2}} .
\end{aligned}
$$

We next derive a matching condition for $\widehat{\Pi}(\Lambda)$ as $\Lambda \rightarrow \infty$. We first note from (97) that if we write $s_{1}^{0}+W_{0}=\sqrt{W_{0}} f(X)$ then

$$
\begin{aligned}
& f(X) \\
& \quad=\frac{1}{2}\left[W_{0}+1-X+\sqrt{\left(W_{0}+1-X\right)^{2}-4 W_{0}}\right], \\
& f\left(X_{L}\right)=\frac{W_{0}+Y_{0}}{1-\sqrt{X_{0}}}=\frac{W_{0}+Y_{0}}{\sqrt{W_{0}}} \Longrightarrow s_{1}^{0}\left(X_{L}\right)=Y_{0} .
\end{aligned}
$$

From (406) we then obtain

$$
\begin{aligned}
f^{\prime}\left(X_{L}\right) & =-\frac{\left(Y_{0}+W_{0}\right)^{2}}{Y_{0}\left(Y_{0}+2 W_{0}\right)}, \\
f^{\prime \prime}\left(X_{L}\right) & =-\frac{2 W_{0}^{5 / 2}\left(Y_{0}+W_{0}\right)^{3}}{Y_{0}^{3}\left(Y_{0}+2 W_{0}\right)^{3}} .
\end{aligned}
$$

From (80) with $Y=0$ we have $\Phi_{X}(X, 0)=-\log [1-f(X)]$ from which we can show that

$$
\begin{aligned}
& \Phi_{X}\left(X_{L}, 0\right)=\Psi_{X}\left(X_{L}, 0\right), \\
& \Phi_{X X}\left(X_{L}, 0\right)=\Psi_{X X}\left(X_{L}, 0\right), \\
& \Phi_{X X X}\left(X_{L}, 0\right) \\
& =\frac{W_{0}\left(W_{0}+Y_{0}\right)^{3}\left[Y_{0}^{3}+3 W_{0} Y_{0}^{2}+4 W_{0}^{2} Y_{0}-2 \sqrt{X_{0}} W_{0}^{5 / 2}\right]}{Y_{0}^{3}\left(2 W_{0}+Y_{0}\right)^{3}\left(\sqrt{X_{0}}-X_{0}-Y_{0}\right)^{2}} .
\end{aligned}
$$

By using the expression for $C_{1}$ in (41) we have

$$
\begin{aligned}
& \Phi\left(X_{L}, 0\right)-\Phi\left(X_{0}, Y_{0}\right)=\Psi\left(X_{L}, 0\right), \\
& \rho^{1 / 3}\left[\Phi_{*}\left(s_{1}^{0}\right)-\Phi_{*}\left(Y_{0}\right)\right]=-\rho^{1 / 3} r_{0} \frac{\left(1-\sqrt{X_{0}}\right)^{2 / 3}}{X_{0}^{1 / 6}} \\
& \cdot \log \left(\frac{s_{1}^{0}+W_{0}}{Y_{0}+W_{0}}\right)=-\rho^{1 / 3} r_{0} \frac{\left(1-\sqrt{X_{0}}\right)^{2 / 3}}{X_{0}^{1 / 6}} \\
& \cdot \log \left(\frac{f(X)}{f\left(X_{L}\right)}\right) \sim-r_{0} \Lambda \frac{\left(1-\sqrt{X_{0}}\right)^{2 / 3}}{X_{0}^{1 / 6}} \frac{f^{\prime}\left(X_{L}\right)}{f\left(X_{L}\right)} \\
& \quad=r_{0} \Lambda \frac{Y_{0}+W_{0}}{Y_{0}\left(Y_{0}+2 W_{0}\right)} \frac{\left(1-\sqrt{X_{0}}\right)^{5 / 3}}{X_{0}^{1 / 6}},
\end{aligned}
$$

$$
\begin{aligned}
& \sqrt{\rho} C_{1} \frac{\left(1-\sqrt{X_{0}}\right)^{1 / 6}}{X_{0}^{1 / 6}\left[\operatorname{Ai}^{\prime}\left(r_{0}\right)\right]^{2}} \\
& \cdot \frac{W_{0}+s_{1}^{0}}{\sqrt{s_{1}^{0}\left(s_{1}^{0}+2 W_{0}\right)}}\left(\frac{\sqrt{W_{0}}}{s_{1}^{0}+W_{0}}\right)^{1 / 2 \sqrt{X_{0}}} \\
& \cdot \sqrt{\sqrt{X_{0}}-X_{0}-s_{1}^{0}} \sim \rho^{-1 / 3} \frac{\left(1-X_{0}-Y_{0}\right)}{\operatorname{Ai}^{\prime}\left(r_{0}\right)}
\end{aligned}
$$




$$
\begin{aligned}
& \cdot \sqrt{2 \pi} \sqrt{\frac{W_{0}+Y_{0}}{Y_{0}+2 W_{0}}} \frac{\left(1-\sqrt{X_{0}}\right)^{5 / 6} X_{0}^{1 / 6}}{\left(\sqrt{X_{0}}-X_{0}-Y_{0}\right)^{3 / 2}} \\
& \cdot \exp \left[-\rho \Phi\left(X_{0}, Y_{0}\right)-\rho^{1 / 3} \Phi_{*}\left(Y_{0}\right)\right] .
\end{aligned}
$$

With (409)-(411) we have obtained the behavior of (96) as $X \downarrow X_{L}$ and thus derived the matching condition

$$
\begin{aligned}
& \widehat{\Pi}(\Lambda) \sim \exp \left[\frac{1}{6} \Phi_{X X X}\left(\mathrm{X}_{L}, 0\right) \Lambda^{3}\right. \\
& \left.\quad+r_{0} \Lambda \frac{Y_{0}+W_{0}}{Y_{0}\left(Y_{0}+2 W_{0}\right)} \frac{\left(1-\sqrt{X_{0}}\right)^{5 / 3}}{X_{0}^{1 / 6}}\right] \frac{1}{\mathrm{Ai}^{\prime}\left(r_{0}\right)} \\
& \quad \sqrt{\frac{W_{0}+Y_{0}}{Y_{0}+2 W_{0}}} \frac{\left(1-\sqrt{X_{0}}\right)^{5 / 6} X_{0}^{1 / 6}}{\left(\sqrt{X_{0}}-X_{0}-Y_{0}\right)^{3 / 2}}, \quad \Lambda \longrightarrow+\infty .
\end{aligned}
$$

Thus (400) and (412) yield the behavior of $\widehat{\Pi}(\Lambda)$ as $\Lambda \rightarrow \pm \infty$, but to determine this function for all $\Lambda$ we must use a third matching condition, to the transition layer in Proposition 18, which applies for $Y-Y_{c}(X)=\rho^{-1 / 3} \eta_{* *}=$ $O\left(\rho^{-1 / 3}\right)$. Thus we let $X \rightarrow X_{L}$ in (191) and let $r \rightarrow \infty$ in (399), in such a way that $\Lambda$ is fixed. Since $\Lambda=\rho^{1 / 3}\left(X-X_{L}\right)$ this means that

$$
\begin{aligned}
\eta_{* *} & =\rho^{1 / 3}\left[Y-Y_{c}(X)\right] \\
& \sim \rho^{1 / 3}\left[\frac{r}{\rho}-Y_{c}^{\prime}\left(X_{L}\right)\left(X-X_{L}\right)\right] \sim-Y_{c}^{\prime}\left(X_{L}\right) \Lambda
\end{aligned}
$$

remains also $O(1)$. From (86) we have

$$
Y_{c}^{\prime}\left(X_{L}\right)=\frac{W_{0}^{3 / 2}\left(Y_{0}+W_{0}\right)}{Y_{0}\left(Y_{0}+2 W_{0}\right)}
$$

We can expand $\rho\left[\Phi\left(X, Y_{c}+\rho^{-1 / 3} \eta_{* *}\right)-\Phi\left(X_{0}, Y_{0}\right)\right]$ in (191) as $X \rightarrow X_{L}$ which is equivalent to expanding $\rho[\Phi(X, Y)-$ $\left.\Phi\left(X_{0}, Y_{0}\right)\right]$ for $(X, Y) \rightarrow\left(X_{L}, 0\right)$ and show that the exponential factors in (191) agree with those in (399), after $r$ ! is approximated by Stirling's formula, so that

$$
\begin{aligned}
\frac{\rho^{r} W_{0}^{r}}{r !} \sim & (2 \pi)^{-1 / 2} r^{-1 / 2} \\
& \cdot \exp \left\{-\rho Y \log Y+\rho Y\left[1+\log \left(W_{0}\right)\right]\right\} .
\end{aligned}
$$

Then using $C_{5}=\rho^{-1 / 3}\left(1-X_{0}-Y_{0}\right)$ we compare algebraic factors in (399) and (191), which yields

$$
\begin{aligned}
& \frac{1}{\sqrt{2 \pi}}\left(1-X_{0}-Y_{0}\right) \rho^{-1 / 3}(\rho Y)^{-1 / 2} \widehat{\Pi}(\Lambda) \\
& \quad \sim\left(1-X_{0}-Y_{0}\right) \rho^{-5 / 6} \frac{1}{\sqrt{2 \pi}} \frac{\left(1-\sqrt{X_{0}}\right)^{5 / 6} X_{0}^{1 / 6}}{\left(\sqrt{X_{0}}-X_{0}-Y_{0}\right)^{3 / 2}}
\end{aligned}
$$

$$
\begin{aligned}
& \sqrt{\frac{W_{0}+Y_{0}}{2 W_{0}+Y_{0}}} \frac{1}{\sqrt{Y_{C}^{\prime}\left(X_{L}\right)\left(X-X_{L}\right)}} \\
& \cdot \exp \left[\frac{1}{6} \Lambda^{3} \Phi_{X X X}\left(X_{L}, 0\right)\right] \frac{1}{2 \pi i} \int_{-i \infty}^{i \infty} \frac{e^{\star \theta}}{\mathrm{Ai}(\theta)} d \theta,
\end{aligned}
$$

where

$$
\star=\Lambda \frac{Y_{0}+W_{0}}{Y_{0}\left(Y_{0}+2 W_{0}\right)} \frac{\left(1-\sqrt{X_{0}}\right)^{5 / 3}}{X_{0}^{1 / 6}} .
$$

In this limit $Y \sim Y_{c}^{\prime}\left(X_{L}\right)\left(X-X_{L}\right)$ and $\Lambda$ is fixed, so that (416) determines $\widehat{\Pi}(\cdot)$ as

$$
\begin{aligned}
\widehat{\Pi}(\Lambda)= & \frac{\left(1-\sqrt{X_{0}}\right)^{5 / 6} X_{0}^{1 / 6}}{\left(\sqrt{X_{0}}-X_{0}-Y_{0}\right)^{3 / 2}} \\
& \cdot \sqrt{\frac{W_{0}+Y_{0}}{2 W_{0}+Y_{0}}} \exp \left[\frac{1}{6} \Lambda^{3} \Phi_{X X X}\left(X_{L}, 0\right)\right] \frac{1}{2 \pi i} \\
& \cdot \int_{-i \infty}^{i \infty} \frac{e^{\star \theta}}{\operatorname{Ai}(\theta)} d \theta .
\end{aligned}
$$

We have thus derived the result in (105). By using the asymptotic results in (192) and (193) (with $\star$ replaced by $-\star$ ) we see immediately that (412) holds. The matching condition in (400) will also hold since

$$
\frac{1}{6} \Phi_{X X X}\left(X_{L}, 0\right)+\frac{1}{3}\left(\frac{\star}{\Lambda}\right)^{3}=\frac{1}{6} \Psi_{X X X}\left(X_{L}, 0\right),
$$

by (417), (405), and (410). This completes our analysis of the scale $r=O(1)$.

5.3. The Boundary Segment $Y=Y_{0}$. We consider the scale $p=\rho\left(Y_{0}-Y\right)=O(1)$ and $0<X<X_{0}$. Now the analysis will be the same for any region $\mathscr{R}_{j}$, and the expansion we construct will hold everywhere except near the corner points $(X, Y)=\left(0, Y_{0}\right)$ and $\left(X_{0}, Y_{0}\right)$. By expanding (3) and the boundary condition in (5) along $r=R$, we find that an asymptotic solution in this range is given by

$$
\pi(k, r) \sim C_{6}(\rho) \frac{\rho^{p}}{p !}\left[Y_{0}\left(e^{\tau_{U}}-1\right)\right]^{p} e^{\rho \Psi\left(X, Y_{0}\right)} \bar{g}(X),
$$

where $\tau_{U}=\tau_{U}(X)$ is given in (128). Again, (420) must contain the factor $\exp \left[\rho \Psi\left(X, Y_{0}\right)\right]$ in order to have a chance of matching to the $\mathscr{D}_{0}$ ray expansion, and this factor determines the geometric factor in $p$. We must only determine $C_{6}(\rho)$ and $\bar{g}(X)$ by asymptotic matching. Also,

As $Y \rightarrow Y_{0}$ we have $A \rightarrow A_{\max }$ and $B \rightarrow-\infty$ in (68).

$$
\Psi_{Y}=-\log (-B)-\tau+o(1), \quad Y \longrightarrow Y_{0}
$$

and from (68) we obtain

$$
-B \sim \frac{Y_{0}\left(1-e^{-\tau_{U}}\right)}{Y_{0}-Y},
$$


where $\tau_{U}=\tau_{U}(X)$ is obtained by solving the first equation in (68) with $A$ replaced by $A_{\max }$. Using (421) and (422) we conclude that

$$
\begin{aligned}
\Psi(X, Y)= & \Psi\left(X, Y_{0}\right)+\left(Y-Y_{0}\right) \log \left(Y_{0}-Y\right)+Y_{0} \\
& -Y+\left(Y_{0}-Y\right) \log \left[Y_{0}\left(e^{\tau_{U}}-1\right)\right] \\
& +o\left(Y_{0}-Y\right), \quad Y \longrightarrow Y_{0} .
\end{aligned}
$$

Expanding $p$ ! by Stirling's formula and comparing the result to

$$
Q(0,0) \rho^{-1 / 2} K(X, Y) e^{\rho \Psi(X, Y)}
$$

as $Y \rightarrow Y_{U}$ we see that we can take $C_{6}=Q(0,0) \sim 1-X_{0}-Y_{0}$ and the matching will hold if

$$
\begin{aligned}
\frac{1}{\sqrt{2 \pi p}} \bar{g}(X) & =\rho^{-1 / 2} \frac{1}{\sqrt{2 \pi\left(Y_{0}-Y\right)}} \bar{g}(X) \\
& \sim \rho^{-1 / 2} K(X, Y), \quad Y \longrightarrow Y_{0} .
\end{aligned}
$$

To expand (70) as $Y \rightarrow Y_{0}$ we note that in this limit $\left(e^{-\tau}-\right.$ $A)^{-1 / 2}$ and $|\Delta|^{-1 / 2}$ are finite, while $\left(e^{-\tau}-B\right)^{-1 / 2} \sim(-B)^{-1 / 2}$ vanishes and $K_{0}(A)$ is singular, due to the factor $Y_{0}+(1-$ $\left.X_{0}-Y_{0}\right) A-A^{2}$ in the denominator. We thus have

$$
\begin{array}{r}
K(X, Y) \sim \frac{1}{\sqrt{-B}}\left(e^{-\tau_{U}}-A_{\max }\right)^{-1 / 2}\left|\Delta_{U}(X)\right|^{-1 / 2} \\
\cdot \sqrt{\frac{Y_{0}}{2 \pi}} \frac{\left(1-A_{\max }\right)^{2}-X_{0}}{1-X_{0}-Y_{0}-2 A_{\max }} \frac{-1}{A-A_{\max }}, \\
Y \longrightarrow Y_{0} .
\end{array}
$$

Here $\Delta_{U}(X)$ is the Jacobian in (71) evaluated along $Y=Y_{0}$, and we also used the identity $A_{\max }\left(A_{\max }-1\right)=Y_{0}-A_{\max }\left(X_{0}+\right.$ $\left.Y_{0}\right)$. From (67) we see that

$$
B \sim \frac{A_{\max }\left(1-A_{\max }-X_{0}\right)}{1-X_{0}-Y_{0}-2 A_{\max }} \frac{1}{A-A_{\max }}
$$

so that with (422) we have, as $Y \uparrow Y_{0}$,

$$
\begin{aligned}
K(X, Y) \sim & \frac{1}{\sqrt{2 \pi}} \frac{1}{\sqrt{Y_{0}-Y}} \sqrt{\frac{1-e^{-\tau_{U}}}{e^{-\tau_{U}}-A_{\max }}} \\
& \cdot \frac{X_{0}-\left(1-A_{\max }\right)^{2}}{1-A_{\max }}\left|\Delta_{U}\right|^{-1 / 2} .
\end{aligned}
$$

Using (428) in (425) we can identify $\bar{g}(X)$ and then (420) becomes the same as (127).

Finally we note that as $X \uparrow X_{0}$, (127) will asymptotically match to (168), the approximation valid for $n, p=O(1)$. For $n \rightarrow \infty$ with $p=O(1)$, (168) can be approximated by (247).
As $X \rightarrow X_{0}$ we have $\tau_{U}(X) \rightarrow 0, \Psi\left(X_{0}, Y_{0}\right)=0, \Psi_{X}\left(X_{0}, Y_{0}\right)=$ $-\log \left(1-A_{\text {max }}\right)$, and $z_{+}(0)\left(1-A_{\max }\right)=1$. Then

$$
\begin{aligned}
Y_{0}\left(e^{\tau_{U}(X)}-1\right) & \sim Y_{0} \tau_{U}^{\prime}\left(X_{0}\right)\left(X-X_{0}\right) \\
& =-\frac{n}{\rho} Y_{0} \tau_{U}^{\prime}\left(X_{0}\right) \\
& =\frac{n}{\rho} \frac{Y_{0}}{\sqrt{\left(1+X_{0}+Y_{0}\right)^{2}-4 X_{0}}}
\end{aligned}
$$

and thus, for $X \rightarrow X_{0}$, (127) reduces to (247). This completes the analysis of the scale $p=R-r=O(1)$.

\section{Approximations near State Space Corners}

We consider the four state space corners. The upper right corner $(X, Y)=\left(X_{0}, Y_{0}\right)$ was analyzed already in Section 4, since this was necessary to completely determine the ray expansion in $\mathscr{D}_{0}$. The analysis of the lower right corner $(X, Y)=\left(X_{0}, 0\right)$ is essentially the same as that for the infinite capacity model in [10]. For regions $\mathscr{R}_{1}$ and $\mathscr{R}_{2}$ the leading terms for $\pi(k, r)$ near this corner are the same as for the infinite capacity model, while for regions $\mathscr{R}_{3}$ and $\mathscr{R}_{4}$ the analysis differs only through the multiplicative constant $C_{1}$. Thus we omit the analysis of these two corners, focusing on the lower and upper left corners, $(0,0)$ and $\left(0, Y_{0}\right)$.

6.1. The Corner $(X, Y)=(0,0)$. For regions $\mathscr{R}_{1} \cup \mathscr{R}_{2} \cup \mathscr{R}_{3}$ the analysis is very similar to that in [10], leading to (132)-(136) in Proposition 13. For region $\mathscr{R}_{4}$ we set

$$
\pi(k, r)=C_{7}(\rho) e^{\rho \Psi(0,0)} \rho^{k+r} Q_{*}(k, r ; \rho),
$$

recalling that $\Psi(0,0)=-X_{0}-Y_{0}+\left(X_{0}+Y_{0}\right) \log \left(X_{0}+Y_{0}\right)$. Dividing the main balance equation in (3) by $\rho$, using (430), and letting $Q_{*}(k, r)$ denote the leading term approximation for $Q_{*}(k, r ; \rho)$, we obtain in the limit $\rho \rightarrow \infty$

$$
\begin{aligned}
Q_{*}(k, r)= & (r+1) Q_{*}(k, r+1) \\
& +(k+1) Q_{*}(k+1, r) ; \quad k \geqslant 1, r \geqslant 0 .
\end{aligned}
$$

Note that (6) implies that (431) holds along $k=0, r \geqslant 0$. Equation (431) has many different solutions, and anything of the form $\alpha_{0}^{k} \beta_{0}^{r} /(k ! r !)$ will be a solution provided that $\alpha_{0}+\beta_{0}=$ 1. Let us write

$$
Q_{*}(k, r)=\frac{1}{k ! r !} \alpha_{0}^{k}\left(1-\alpha_{0}\right)^{r}
$$

and we will show by asymptotic matching that only one value of $\alpha_{0}$ is needed, and the matching will also determine the appropriate value. For regions $\mathscr{R}_{1} \cup \mathscr{R}_{2} \cup \mathscr{R}_{3}$ the same argument was used in [10] to determine $\alpha_{0}$ as $1-W_{0}=2 \sqrt{X_{0}}-$ $X_{0}$, leading to (136). For region $\mathscr{R}_{4}$ we can asymptotically match (430) to (117) (then $r \rightarrow \infty$ with $k=O(1)$ ), to (98) (then $k \rightarrow \infty$ with $r=O(1)$ ), or to the $\mathscr{D}_{0}$ ray expansion (then $k, r \rightarrow \infty$ with $k / r$ fixed). We discuss only the first two matchings, as the third will lead to the same conclusion. 
For fixed $k$ and $r \rightarrow \infty,(430)$ and (432) become

$$
\begin{aligned}
& \pi(k, r) \sim C_{7}(\rho) \frac{\rho^{k+r}}{k ! r !} e^{\rho \Psi(0,0)} \alpha_{0}^{k}\left(1-\alpha_{0}\right)^{r} \sim \frac{C_{7}(\rho)}{\sqrt{2 \pi \rho Y}} \\
& . \frac{\rho^{k}}{k !} \alpha_{0}^{k} \exp \{\rho[\Psi(0,0)-Y \log Y+Y \\
& \left.\left.+Y \log \left(1-\alpha_{0}\right)\right]\right\},
\end{aligned}
$$

where the last formula holds in the matching region where $r \rightarrow \infty$ but $Y=r / \rho \rightarrow 0$, and we wrote the expression in terms of $Y$. To expand (117) as $Y \rightarrow 0$, we observe from (118) that $A_{1}(0)=1-X_{0}-Y_{0}$. Then comparing the geometric factors in $k$, in (117) and (433), we must have

$$
\alpha_{0}=\frac{A_{1}(0)\left(A_{1}(0)+X_{0}-2\right)+1}{1-A_{1}(0)}=Y_{0}+\frac{X_{0}}{X_{0}+Y_{0}} .
$$

From (119) we find that as $Y \rightarrow 0$

$$
\begin{aligned}
\Psi(0, Y)= & \Psi(0,0)+Y-Y \log Y \\
& +Y \log \left[\frac{Y_{0}\left(1-X_{0}-Y_{0}\right)}{X_{0}+Y_{0}}\right]+o(Y)
\end{aligned}
$$

which implies that $1-\alpha_{0}=Y_{0}\left(1-X_{0}-Y_{0}\right) /\left(X_{0}+Y_{0}\right)$, consistent with (434). The matching also implies that

$$
\frac{C_{7}(\rho)}{\sqrt{2 \pi \rho Y}} \sim Q(0,0) \frac{\sqrt{A_{1}(0)} \sqrt{A_{1}(0)\left(A_{1}(0)+X_{0}-2\right)+1}\left\{\left[1-A_{1}(0)\right]^{2}-X_{0}\right\}}{\sqrt{Y} \sqrt{\left|\Delta_{L}(0)\right|}\left[Y_{0}-A_{1}(0)\left(X_{0}+Y_{0}\right)\right]} .
$$

After some calculation we find from (120) that

$$
-\Delta_{L}(0)=\left(1-X_{0}-Y_{0}\right) \frac{X_{0}+Y_{0}\left(X_{0}+Y_{0}\right)}{X_{0}+Y_{0}}
$$

and thus

$$
C_{7}(\rho) \sim \sqrt{\rho} \sqrt{2 \pi\left(X_{0}+Y_{0}\right)} Q(0,0) .
$$

We have thus derived (137), since $Q(0,0) \sim 1-X_{0}-Y_{0}$ for region $\mathscr{R}_{4}$.

The same conclusions follow by matching to (98), as then, for fixed $r$ and $k \rightarrow \infty$, (430) with (432) becomes

$$
\begin{aligned}
& \pi(k, r) \sim \frac{C_{7}(\rho)}{\sqrt{2 \pi \rho X}} \frac{\rho^{r}}{r !}\left(1-\alpha_{0}\right)^{r} \\
& \cdot \exp \left\{\rho\left[\Psi(0,0)-X \log X+X+X \log \left(\alpha_{0}\right)\right]\right\} .
\end{aligned}
$$

We expand (98) as $X \rightarrow 0$. We have $A_{0}(0)=1-X_{0}-Y_{0}$ and from (100) find that

$$
A_{0}^{\prime}(0)=-\frac{Y_{0}}{X_{0}+Y_{0}\left(X_{0}+Y_{0}\right)} .
$$

Then also $B_{0}(0)=1-X_{0}-Y_{0}$ and, using (101),

$$
B_{0}^{\prime}(0)=-\frac{\left(X_{0}+Y_{0}\right)^{2}-X_{0}}{X_{0}+Y_{0}\left(X_{0}+Y_{0}\right)}
$$

We have, as $X \rightarrow 0$,

$$
\begin{aligned}
& \Psi_{X}(X, 0)+\log X=\log X-\log \left[1-\frac{A_{0}(X)}{B_{0}(X)}\right] \\
& \quad=\log \left[B_{0}(X)\right]-\log \left[\frac{B_{0}(X)-A_{0}(X)}{X}\right] \longrightarrow \\
& \log \left[B_{0}(0)\right]-\log \left[B_{0}^{\prime}(0)-A_{0}^{\prime}(0)\right] \\
& \quad=\log \left[Y_{0}+\frac{X_{0}}{X_{0}+Y_{0}}\right] .
\end{aligned}
$$

By using (442) to infer the small $X$ behavior of $\Psi(X, 0)$ and comparing the result to the exponential part of (439), we again conclude that $\alpha_{0}$ is as in (434). To expand $g(X)$ in (102) as $X \rightarrow 0$ we note that it is singular due to the factor

$$
\begin{aligned}
& {\left[B_{0}(X)-A_{0}(X)\right]^{-1 / 2}} \\
& \quad \sim X^{-1 / 2}\left[B_{0}^{\prime}(0)-A_{0}^{\prime}(0)\right]^{-1 / 2} .
\end{aligned}
$$

Then after some calculation, using (440) and (441) we find that $g(X)$ has the expansion in (103) as $X \rightarrow 0$. Expanding (98) as $X \rightarrow 0$ and comparing the result to (439), noting that $Y_{0} B_{0}(0) /\left[1-B_{0}(0)\right]=1-\alpha_{0}$, regain the expression in (438) for $C_{7}(\rho)$. We can also easily verify that (137) matches to the $\mathscr{D}_{0}$ ray expansion, by expanding the latter for $X, Y \rightarrow 0$ along lines of constant slope $Y / X$.

6.2. The Corner $(X, Y)=\left(0, Y_{0}\right)$. Now we use the variables $k$ and $p=R-r$, with

$$
\pi(k, r)=C_{8}(\rho) e^{\rho \Psi\left(0, Y_{0}\right)} \rho^{k+p} \widehat{Q}(k, p ; \rho) .
$$

Near this corner only the balance equations in (3), (5), (6), and (7) apply. Using (444) in (3) we obtain

$$
\begin{aligned}
(\rho+ & \left.\rho Y_{0}+k-p\right) \widehat{Q}(k, p ; \rho) \\
= & {\left[Y_{0}+(1-p) \rho^{-1}\right] \widehat{Q}(k, p-1 ; \rho) } \\
& \quad+\rho(k+1) \widehat{Q}(k+1, p ; \rho)+\widehat{Q}(k-1, p ; \rho) .
\end{aligned}
$$

Letting $\widehat{Q}(k, p)$ be the limiting form of $\widehat{Q}(k, p ; \rho)$ as $\rho \rightarrow \infty$, (445) leads to

$$
\left(1+Y_{0}\right) \widehat{Q}(k, p)=(k+1) \widehat{Q}(k+1, p)
$$


whose most general solution is

$$
\widehat{Q}(k, p)=\frac{\left(1+Y_{0}\right)^{k}}{k !} \bar{Q}(p) .
$$

Equations (5) -(7) provide, in the limit $\rho \rightarrow \infty$, no additional information. To determine $\bar{Q}(p)$ in (447) we use asymptotic matching to the boundary layer expansion in (127), which applies for $p=O(1)$ and $0<X<X_{0}$.

As $X \rightarrow 0$ we have $\tau_{U}(X) \rightarrow \tau_{U}(0)=-\log \left(A_{\max }\right)$, and then $\Psi\left(0, Y_{0}\right)$ is as in (131). Also, from (68) with $(A, \tau)$ replaced by $\left(A_{\max }, \tau_{U}\right)$ we find that

$$
1-A_{\max } e^{\tau_{U}(X)} \sim \frac{X}{1+Y_{0}}, \quad X \longrightarrow 0
$$

and then $\Psi_{X}\left(X, Y_{0}\right)+\log X \rightarrow \log \left(1+Y_{0}\right)$ as $X \rightarrow 0$. Using the above in (127) we find that for $X \rightarrow 0$ we have

$$
\begin{gathered}
\pi(k, r) \sim Q(0,0) e^{\rho \Psi\left(0, Y_{0}\right)} e^{\rho[X \log X-X]} e^{\rho X \log \left(1+Y_{0}\right)} \\
\cdot \frac{1}{p !}\left[Y_{0}\left(\frac{1}{A_{\max }}-1\right)\right]^{p} \\
\cdot \sqrt{\frac{1-A_{\max }}{A_{\max }}}\left[\left(1-X_{0}-Y_{0}\right)^{2}+4 Y_{0}\right]^{1 / 4}
\end{gathered}
$$

By comparing (449) to the large $k$ expansion of (444), with (447), we can take

$$
C_{8}(\rho)=\sqrt{2 \pi \rho} Q(0,0)
$$

and then $\bar{Q}(\cdot)$ is determined as

$$
\begin{aligned}
\bar{Q}(p)= & \frac{1}{p !}\left(Y_{0} \frac{1-A_{\max }}{A_{\max }}\right)^{p} \\
& \cdot \sqrt{\frac{1-A_{\max }}{A_{\max }}}\left[\left(1-X_{0}-Y_{0}\right)^{2}+4 Y_{0}\right]^{1 / 4}
\end{aligned}
$$

We have thus established the result in (130).

We conclude by showing that (130), for $p \rightarrow \infty$ and fixed $k$, matches asymptotically to (117), for $Y \uparrow Y_{0}$ and fixed $k$. In this limit, (117) is singular due to the factor $1 / \sqrt{Y_{0}-Y}=$ $\sqrt{\rho / p}$. First we note from (118) that $A_{1}\left(Y_{0}\right)=A_{\max }$ and from (119) we get

$$
\Psi_{Y}(0, Y)=\log \left[\frac{\left(Y_{0}-Y\right) A_{1}(Y)}{Y\left(1-A_{1}(Y)\right)}\right]
$$

and thus

$$
\begin{aligned}
& \Psi(0, Y)-\Psi\left(0, Y_{0}\right) \\
& =\left(Y-Y_{0}\right) \log \left(Y_{0}-Y\right)+Y_{0}-Y \\
& \quad+\left(Y-Y_{0}\right) \log \left[\frac{A_{\max }}{1-A_{\max }} \frac{1}{Y_{0}}\right]+o\left(Y-Y_{0}\right) .
\end{aligned}
$$

Thus,

$$
\begin{array}{r}
e^{\rho \Psi(0, Y)} \sim e^{\rho \Psi\left(0, Y_{0}\right)}\left[\frac{1-A_{\max }}{A_{\max }} Y_{0}\right]^{p}\left(\frac{\rho}{p}\right)^{p} e^{p}, \\
Y \longrightarrow Y_{0} .
\end{array}
$$

After some calculation we find from (120) that

$$
\begin{aligned}
-\Delta_{L}\left(Y_{0}\right) & =\left(1+Y_{0}\right)\left[\frac{X_{0}}{\left(1-A_{\max }\right)^{2}}-1\right]\left(1-A_{\max }\right) \\
& =\left(1+Y_{0}\right)\left[\left(1-X_{0}-Y_{0}\right)^{2}+4 Y_{0}\right]^{1 / 2}
\end{aligned}
$$

and also

$$
\begin{aligned}
& \frac{\sqrt{A_{\max }} \sqrt{A_{\text {max }}\left(A_{\text {max }}+X_{0}-2\right)+1}\left[\left(1-A_{\max }\right)^{2}-X_{0}\right]}{Y_{0}-\left(X_{0}+Y_{0}\right) A_{\max }} \\
& =\frac{\sqrt{A_{\max }\left(A_{\max }+X_{0}-2\right)+1}}{\sqrt{A_{\max }}\left(1-A_{\max }\right)}\left[\left(1-A_{\max }\right)^{2}-X_{0}\right] \\
& =\sqrt{1+Y_{0}} \sqrt{\frac{1-A_{\max }}{A_{\max }}} \sqrt{\left(1-X_{0}-Y_{0}\right)^{2}+4 Y_{0}} .
\end{aligned}
$$

With (454)-(456) the expansion of (117) agrees precisely with the large $p$ behavior of (444), with (447), (450), and (451). This completes the matching verification.

\section{Approximations near Transition Layers}

We analyze the vicinities of the curves where the regions $\mathscr{D}_{0}$, $\mathscr{D}_{+}$, and $\mathscr{D}_{-}$meet. From Figures 3-6 we see that, for $\mathscr{R}_{1}, \mathscr{D}_{0}$ meets $\mathscr{D}_{+}$along the curve $Y=\tilde{Y}(X)$. For regions $\mathscr{R}_{2} \cup \mathscr{R}_{3}, \mathscr{D}_{0}$ also meets $\mathscr{D}_{+}$, while $\mathscr{D}_{+}$and $\mathscr{D}_{-}$meet along $Y=Y_{*}(X)$. For region $\mathscr{R}_{4}, \mathscr{D}_{0}$ and $\mathscr{D}_{-}$meet along $Y=Y_{c}(X)$. The analysis for $Y=Y_{*}(X)$, with the scaling $Y-Y_{*}(X)=O\left(\rho^{-1 / 3}\right)$, is carried out in [10] and we omit it here. For $\mathscr{R}_{2}$ the analysis is exactly as in [10], while for $\mathscr{R}_{2} \cap \mathscr{R}_{3}$ and $\mathscr{R}_{3}$ the $\mathscr{D}_{+}$and $\mathscr{D}_{-}$ray expansions must be multiplied by the appropriate constants $C$ and $C_{1}$, but the analysis is otherwise unchanged. We thus obtain the result in (188).

7.1. Transition Layer near $Y=Y_{c}(X)$, Region $\mathscr{R}_{4}$. This layer arises only for parameter region $\mathscr{R}_{4}$. We set $\rho^{1 / 3}\left[Y-Y_{c}(X)\right]=$ $\eta_{* *}=O(1)$ and use $\left(X, \eta_{* *}\right)$ as the variables. Let us write $L(X, Y)$ in the ray expansion in (79) as

$$
\begin{aligned}
& L(X, Y)=\frac{\left(W_{0}+s_{1}\right)^{2}}{\sqrt{Y} \sqrt{W_{0}+Y}} \frac{1}{\sqrt{s_{1}-Y}} \\
& \cdot \frac{1}{\sqrt{2 W_{0}+s_{1}+Y}}\left[1-\frac{\sqrt{W_{0}}\left(W_{0}+s_{1}\right)}{W_{0}+Y}\right]^{-1 / 2} \\
& \cdot L_{0}\left(s_{1}\right) \equiv L_{1}(X, Y) L_{0}\left(s_{1}\right),
\end{aligned}
$$


where

$$
\begin{gathered}
L_{0}\left(s_{1}\right)=\frac{1}{2 \pi}\left[\operatorname{Ai}^{\prime}\left(r_{0}\right)\right]^{-2} X_{0}^{-1 / 6}\left(1-\sqrt{X_{0}}\right)^{-5 / 6} \\
\cdot \frac{\left(s_{1}+W_{0}\right) \sqrt{X_{0}}-s_{1}}{\sqrt{W_{0}+s_{1}}} \\
\cdot \exp \left[-\left(\frac{1}{2}+\frac{1}{2 \sqrt{X_{0}}}\right) \log \left(\frac{W_{0}+s_{1}}{1-\sqrt{X_{0}}}\right)\right] .
\end{gathered}
$$

The scaling $Y-Y_{c}(X)=O\left(\rho^{-1 / 3}\right)$ corresponds to rays that have $s_{1} \approx Y_{0}$ and more precisely $s_{1}-Y_{0}=O\left(\rho^{-1 / 3}\right)$. We thus set

$$
s_{1}=Y_{1}+\rho^{-1 / 3} \widetilde{s}_{1} .
$$

In view of (75) and (86) we have

$$
\frac{Y_{c}(X)+W_{0}}{Y_{0}+W_{0}}=\frac{Y_{*}(X)+W_{0}}{\sqrt{W_{0}}}
$$

which relates the two curves $Y_{c}$ and $Y_{*}$. From the definition of $s_{1}$ in (81) we then have

$$
s_{1}+W_{0}=Y_{0}+W_{0}+\rho^{-1 / 3} \widetilde{s}_{1}=\frac{\sqrt{W_{0}}\left(Y+W_{0}\right)}{Y_{*}(X)+W_{0}}
$$

so that

$$
\begin{aligned}
\widetilde{s}_{1} & =\rho^{-1 / 3} \sqrt{W_{0}}\left[\frac{Y+W_{0}}{Y_{*}(X)+W_{0}}-\frac{Y_{c}(X)+W_{0}}{Y_{*}(X)+W_{0}}\right] \\
& =\rho^{-1 / 3} \frac{\sqrt{W_{0}}}{Y_{*}(X)+W_{0}}\left[Y-Y_{c}(X)\right] \\
& =\frac{Y_{0}+W_{0}}{Y_{c}(X)+W_{0}} \eta_{* *} .
\end{aligned}
$$

Consider the balance equation (17) on the scale $\eta_{* *}=$ $O(1)$, with $0<X<X_{0}$. An asymptotic solution is given by

$$
C_{1} e^{\rho \Phi(X, Y)} e^{\rho^{1 / 3} \Phi_{1}(X, Y)} L(X, Y) \mathscr{L}\left(X, \eta_{* *}\right),
$$

where we can replace $Y$ by $Y_{c}(X)+\rho^{-1 / 3} \eta_{* *}$, so the expansion is in terms of $X$ and $\eta_{* *}$. We can also view $\mathscr{L}$ as being a function of the ray variables $t$ and $\widetilde{s}_{1}$. But then the product $L \mathscr{L}$ will satisfy the transport equation in (198). Since $L$ is a particular solution, $\mathscr{L}$ must be constant along a ray and thus a function of $\widetilde{s}_{1}$ but not $t$, so in view of (462) we write

$$
\mathscr{L}\left(X, \eta_{* *}\right)=\mathscr{L}_{0}\left(\frac{Y_{0}+W_{0}}{Y_{c}(X)+W_{0}} \eta_{* *}\right) .
$$

We thus write the expansion in the transition layer as

$$
\begin{aligned}
& \pi(k, r) \sim C_{1} L\left(X, Y_{c}(X)\right) \mathscr{L}_{0}\left(\frac{Y_{0}+W_{0}}{Y_{c}(X)+W_{0}} \eta_{* *}\right) \\
& \cdot \exp \left[\rho \Phi\left(X, Y_{c}(X)+\rho^{-1 / 3} \eta_{* *}\right)\right. \\
& \left.+\rho^{1 / 3} \Phi\left(X, Y_{c}(X)+\rho^{-1 / 3} \eta_{* *}\right)\right],
\end{aligned}
$$

where we used $L(X, Y) \sim L\left(X, Y_{c}(X)\right)$. If (465) is to asymptotically match to the $\mathscr{D}_{-}$ray expansion we must have

$$
\mathscr{L}_{0}(-\infty)=1
$$

Using the fact that $\Phi_{1}(X, Y)=\Phi_{*}\left(s_{1}\right)$ we have

$$
\begin{aligned}
\rho^{1 / 3} & {\left[\Phi_{*}\left(s_{1}\right)-\Phi\left(Y_{0}\right)\right] } \\
= & \rho^{1 / 3} r_{0}(1-\sqrt{X})^{2 / 3} X_{0}^{-1 / 6} \log \left(\frac{W_{0}+Y_{0}}{W_{0}+s_{1}}\right) \\
& \sim-\frac{r_{0} \eta_{* *}\left(1-\sqrt{X_{0}}\right)^{2 / 3}}{X_{0}^{1 / 6}\left[W_{0}+Y_{c}(X)\right]} .
\end{aligned}
$$

Next we examine the ray expansion in $\mathscr{D}_{0}$ near the curve $Y=Y_{c}(X)$. This will yield a matching condition for $\mathscr{L}_{0}(\cdot)$ as $\eta_{* *} \rightarrow+\infty$. We can easily establish the following continuity conditions between $\Psi(X, Y)$ and $\Phi(X, Y)$ across $Y=Y_{c}(X)$

$$
\begin{aligned}
\Psi\left(X, Y_{c}(X)\right) & =\Phi_{*}\left(X, Y_{c}(X)\right)-\Phi\left(X_{0}, Y_{0}\right), \\
\Psi_{Y}\left(X, Y_{c}(X)\right) & =\Phi_{Y}\left(X, Y_{c}(X)\right), \\
\Psi_{Y Y}\left(X, Y_{c}(X)\right) & =\Phi_{Y Y}\left(X, Y_{c}(X)\right),
\end{aligned}
$$

where we recall that $Y=Y_{c}(X)$ corresponds to the $\mathscr{D}_{0}$ ray with $A=1-\sqrt{X_{0}}, B=W_{0} /\left(W_{0}+Y_{0}\right)$. To this end we note that

$$
\begin{aligned}
\Psi_{Y}(X, Y)= & \log \left[B+(1-B) \frac{Y}{Y_{0}}\right] \\
& -\log \left[(1-B) \frac{Y}{Y_{0}}\right],
\end{aligned}
$$

where we expressed $\tau$ in terms of $B$ and $Y$ using (68). Along $Y=Y_{c}$ we have $\partial B / \partial Y=0$ so that from (469) we obtain

$$
\begin{aligned}
\Psi_{Y Y}= & \frac{Y_{0}}{1-B} \frac{1}{Y_{0} B+(1-B) Y} \frac{\partial B}{\partial Y}+\frac{1-B}{Y_{0} B+(1-B) Y} \\
& -\frac{1}{Y}
\end{aligned}
$$

and hence

$$
\Psi_{Y Y}\left(X, Y_{c}(X)\right)=-\frac{W_{0}}{Y_{c}\left(Y_{c}+W_{0}\right)} .
$$

From the relation between $B$ and $A$ in (67) we have

$$
\frac{Y_{0}}{(1-B)^{2}} \frac{\partial B}{\partial Y}=\left[1-\frac{X_{0}}{(1-A)^{2}}\right] \frac{\partial A}{\partial Y}
$$

and thus

$$
\frac{\partial^{2} B}{\partial Y^{2}}=-\frac{2 Y_{0}}{\sqrt{X_{0}}\left(Y_{0}+W_{0}\right)^{2}}\left(\frac{\partial A}{\partial Y}\right)^{2},
$$

at $Y=Y_{c}(X)$. 
It also follows from (470) that

$$
\begin{aligned}
\Psi_{Y Y Y} & \left(X, Y_{c}(X)\right) \\
= & \frac{1}{Y_{c}^{2}}-\frac{1}{\left(W_{0}+Y_{c}\right)^{2}} \\
& +\frac{\left(Y_{0}+W_{0}\right)^{2}}{Y_{0}\left(Y_{c}+W_{0}\right)} \frac{\partial^{2} B}{\partial Y^{2}}\left(X, Y_{c}(X)\right) .
\end{aligned}
$$

Eliminating $\tau$ in (68) leads to

$$
\begin{aligned}
& (X+Y-1)\left[B+(1-B) \frac{Y}{Y_{0}}\right]+A \\
& \quad=\left(A+X_{0}+Y_{0}-1\right)\left[B+(1-B) \frac{Y}{Y_{0}}\right]^{2} .
\end{aligned}
$$

By implicit differentiation of (475) we obtain

$$
\frac{\partial A}{\partial Y}\left(X, Y_{c}(X)\right)=\frac{\sqrt{W_{0}}}{W_{0}+Y_{c}(X)}
$$

and then using (473) in (474) leads to

$$
\begin{aligned}
\Psi_{Y Y Y}\left(X, Y_{c}(X)\right)= & \frac{1}{Y_{c}^{2}}-\frac{1}{\left(W_{0}+Y_{c}\right)^{2}} \\
& -\frac{2 W_{0}}{\sqrt{X_{0}}} \frac{1}{\left(W_{0}+Y_{c}\right)^{3}} \\
= & \Phi_{Y Y Y}\left(X, Y_{c}(X)\right) \\
& -\frac{2 W_{0}}{\sqrt{X_{0}}} \frac{1}{\left(W_{0}+Y_{c}\right)^{3}} .
\end{aligned}
$$

Combining (468) with (477) we have

$$
\begin{aligned}
& \rho\left\{\Psi\left(X, Y_{c}+\rho^{-1 / 3} \eta_{* *}\right)\right. \\
& \left.-\left[\Phi\left(X, Y_{c}+\rho^{-1 / 3} \eta_{* *}\right)-\Phi\left(X_{0}, Y_{0}\right)\right]\right\} \sim-\frac{1}{3} \\
& \quad \cdot \frac{(1-\sqrt{X})^{2}}{\sqrt{X_{0}}}\left(\frac{\eta_{* *}}{W_{0}+Y_{c}(X)}\right)^{3} . \\
& \text { As } Y \rightarrow Y_{c}(X), e^{-\tau} \rightarrow\left(W_{0}+Y_{c}\right) /\left(W_{0}+Y_{0}\right), \\
& \left(e^{-\tau}-A\right)\left(e^{-\tau}-B\right) \\
& \sim \frac{Y_{c}}{\left(Y_{0}+W_{0}\right)^{2}}\left[Y_{c}+\sqrt{X_{0}} W_{0}-\sqrt{W_{0}} Y_{0}\right], \\
& -\Delta \sim \frac{Y_{0}-Y_{c}}{W_{0}+Y_{0}}\left(Y_{0}+Y_{c}+2 W_{0}\right) .
\end{aligned}
$$

The function $K_{0}(A)$ vanishes as $A \rightarrow 1-\sqrt{X_{0}}$, in view of the factor $\left[(1-A)^{2}-X_{0}\right]$, and we have

$$
\begin{aligned}
& K_{0}(A) \\
& \quad \sim \frac{2 \sqrt{Y_{0}}\left(1-\sqrt{X_{0}}\right)^{2}}{\sqrt{2 \pi}\left(Y_{0}+W_{0}\right)\left(\sqrt{X_{0}}-X_{0}-Y_{0}\right)\left(W_{0}+Y_{c}\right)} \\
& \quad \cdot \rho^{-1 / 3} \eta_{* *}, \quad Y \longrightarrow Y_{c} .
\end{aligned}
$$

Combining (479) and (480) leads to, for $Y \rightarrow Y_{c}(s)$,

$$
\begin{array}{rl}
C_{0} K(X, Y) \sim 2 & 2\left(1-X_{0}-Y_{0}\right) \rho^{-5 / 6} \eta_{* *} \\
& \cdot \frac{\left(1-\sqrt{X_{0}}\right)^{2} \sqrt{Y_{0}} \sqrt{W_{0}+Y_{0}}}{\sqrt{Y_{0}-Y_{c}} \sqrt{Y_{c}+Y_{0}+2 W_{0}}} \frac{1}{\sqrt{2 \pi}} \\
& \cdot \frac{1}{W_{0}+Y_{c}} \frac{1}{\sqrt{X_{0}}-X_{0}-Y_{0}} \\
& \cdot \frac{1}{\sqrt{Y_{c}+W_{0}-\sqrt{W_{0}}\left(W_{0}+Y_{0}\right)}} .
\end{array}
$$

Using the expression in (41) for $C_{1}$ and the continuity conditions in (468) and (477), we compare (465) to the $\mathscr{D}_{0}$ ray expansion as $Y \rightarrow Y_{c}(X)$ to conclude that

$$
\begin{aligned}
& \mathscr{L}_{0}(\star) \sim 2 \mathrm{Ai}^{\prime}\left(r_{0}\right)\left(1-\sqrt{X_{0}}\right)^{2 / 3} X_{0}^{-1 / 6} \frac{\star}{Y_{0}+W_{0}} \\
& \cdot \exp \left[r_{0} \frac{\left(1-\sqrt{X_{0}}\right)^{2 / 3}}{X_{0}^{1 / 6}} \frac{\star}{Y_{0}+W_{0}}\right. \\
& \left.-\frac{1}{3} \frac{\left(1-\sqrt{X_{0}}\right)^{2}}{\sqrt{X_{0}}}\left(\frac{\star}{Y_{0}+W_{0}}\right)^{3}\right], \quad \star \longrightarrow+\infty .
\end{aligned}
$$

Thus (466) and (482) give the behaviors of $\mathscr{L}_{0}(\star)$ as $\star \rightarrow$ $\pm \infty$. But to determine $\mathscr{L}_{0}(\cdot)$ completely we must use asymptotic matching to the corner approximation in (169), which applies on the $(\nu, T)$ scale. We thus expand (465) for $X \rightarrow X_{0}$ and asymptotically match this to (169), expanding the latter for $v \rightarrow \infty, T \rightarrow \infty$ but with $T-\sqrt{v}=O(1)$. In this limit we have

$$
\begin{aligned}
& \frac{1}{2 \pi i} \int_{-i \infty}^{i \infty} \frac{\operatorname{Ai}(\nu+\theta)}{\operatorname{Ai}(\theta)} e^{\theta T} d \theta \\
& \quad \frac{e^{-(2 / 3) \nu^{3 / 2}}}{2 \sqrt{\pi} \nu^{1 / 4}}\left[\frac{1}{2 \pi i} \int_{-i \infty}^{i \infty} \frac{e^{(T-\sqrt{\nu}) \theta}}{\operatorname{Ai}(\theta)} d \theta\right] .
\end{aligned}
$$

Recalling that $X_{0}-X=\rho^{-2 / 3} v_{1}$ we have

$$
Y_{0}-Y_{c}(X) \sim \frac{Y_{0}+W_{0}}{W_{0}^{1 / 4}} \rho^{-1 / 3} \sqrt{\nu_{1}}
$$


and then

$$
\begin{aligned}
\eta_{* *} & =\rho^{1 / 3}\left[Y-Y_{c}(X)\right]=-T_{1}+\rho^{1 / 3}\left[Y_{0}-Y_{c}(X)\right] \\
& \sim \frac{\left(Y_{0}+W_{0}\right) X_{0}^{1 / 6}}{\left(1-\sqrt{X_{0}}\right)^{2 / 3}}(\sqrt{\nu}-T),
\end{aligned}
$$

$$
(X, Y) \longrightarrow\left(X_{0}, Y_{0}\right)
$$

Apart from the exponential factor

$$
\exp \left\{\rho\left[\Phi\left(X, Y_{c}(X)+\rho^{-1 / 3} \eta_{* *}\right)-\Phi\left(X_{0}, Y_{0}\right)\right]\right\},
$$

as $Y \rightarrow Y_{c}$ the expression in (465) becomes

$$
\begin{aligned}
& \left(1-X_{0}-Y_{0}\right) \rho^{-5 / 6} \frac{1}{\sqrt{2 \pi}}\left[\mathrm{Ai}^{\prime}\left(r_{0}\right)\right]^{-1} \\
& \cdot \frac{\left(1-\sqrt{X_{0}}\right)^{4 / 3} X_{0}^{-1 / 12}}{\sqrt{2\left(W_{0}+Y_{0}\right)}\left(\sqrt{X_{0}}-X_{0}-Y_{0}\right)}\left[\frac{Y_{0}+W_{0}}{W_{0}^{1 / 4}}\right. \\
& \left.\cdot \rho^{-1 / 3} \sqrt{\nu_{1}}\right]^{-1 / 2} \exp \left[-r_{0}(\sqrt{\nu}-T)\right] \\
& \cdot \mathscr{L}_{0}\left(\frac{\left(Y_{0}+W_{0}\right) X_{0}^{1 / 6}}{\left(1-\sqrt{X_{0}}\right)^{2 / 3}}(\sqrt{\nu}-T)\right) .
\end{aligned}
$$

Here we note that $L_{1}\left(X, Y_{c}(X)\right)$ is singular as $X \rightarrow X_{0}$, in view of the factor $\left(s_{1}-Y_{c}\right)^{-1 / 2}$, and in this limit

$$
\begin{aligned}
s_{1}-Y_{c} & =s_{1}-Y_{c}+Y_{0}-Y_{c}(X) \\
& \sim \frac{Y_{0}+W_{0}}{W_{0}^{1 / 4}} \sqrt{X_{0}-X} .
\end{aligned}
$$

The limit of (169) as $T, v \rightarrow \infty$, using (483), apart from some exponential factors, which will automatically match those from (486), is given by

$$
\begin{gathered}
\rho^{-2 / 3} \frac{\left(1-\sqrt{X_{0}}\right)^{5 / 3}\left(1-X_{0}-Y_{0}\right)}{\left(Y_{0}+W_{0}\right)\left(\sqrt{X_{0}}-X_{0}-Y_{0}\right)} \\
\cdot \frac{v^{-1 / 4}}{2 \sqrt{\pi}}\left[\frac{1}{2 \pi i} \int_{-i \infty}^{i \infty} \frac{e^{(T-\sqrt{v} \theta}}{\operatorname{Ai}(\theta)} d \theta\right] .
\end{gathered}
$$

Comparing (487) with (489) determines $\mathscr{L}_{0}(\cdot)$ as

$$
\begin{aligned}
\mathscr{L}_{0} & \left(\frac{\left(Y_{0}+W_{0}\right) X_{0}^{1 / 6}}{\left(1-\sqrt{X_{0}}\right)^{2 / 3}} \mathcal{U}\right) \\
= & \frac{\operatorname{Ai}^{\prime}\left(r_{0}\right) e^{r_{0}} \mathcal{U}}{2 \pi i} \int_{-i \infty}^{i \infty} \frac{e^{-\theta \mathscr{U}}}{\operatorname{Ai}(\theta)} d \theta .
\end{aligned}
$$

With (490), (465) becomes the same as (191), so we have established Proposition 8. Note also that (490) is consistent with (466), since for $\mathcal{U} \rightarrow-\infty$ the asymptotics of the contour integral are determined by the pole at $\theta=r_{0}<0$. For
$\mathcal{U} \rightarrow+\infty$ a standard saddle point calculation shows that (490) is asymptotic to

$$
\operatorname{Ai}^{\prime}\left(r_{0}\right) e^{r_{0} \mathscr{U}}(2 \mathscr{U}) e^{-\mathcal{U}^{3} / 3}
$$

which is consistent with (482).

7.2. Transition Layer near $Y=Y_{c}(X)$, Region $\mathscr{R}_{3} \cap \mathscr{R}_{4}$. We take $X_{0}+Y_{0}=\sqrt{X_{0}}+\rho^{-1 / 3} \delta_{*}$. Now the curves $\tilde{Y}(X), Y_{*}(X)$, and $Y_{c}(X)$ are all close to each other, coinciding if $\delta_{*}=0$. Most of the analysis closely parallels that in $\mathscr{R}_{4}$, so we include here fewer of the details. We again use the variables $X$ and $\eta_{* *}$ to find that in the transition layer

$$
\begin{aligned}
& \pi(k, r) \\
& \sim \exp \left\{\rho\left[\Phi\left(X, Y_{c}+\rho^{-1 / 3} \eta_{* *}\right)-\Phi\left(X_{0}, Y_{0}\right)\right]\right\} \\
& \cdot \exp \left[-r_{0} \frac{\left(1-\sqrt{X_{0}}\right)^{2 / 3}}{X_{0}^{1 / 6}\left(W_{0}+Y_{c}\right)} \eta_{* *}\right] \\
& \cdot \rho^{-1 / 2} \mathscr{L}_{1}\left(\frac{Y_{0}+W_{0}}{Y_{c}+W_{0}} \eta_{* *}\right) \frac{1}{Y_{c}^{0}(X)} \\
& \cdot \frac{1}{\sqrt{\sqrt{X_{0}}-X_{0}-Y_{c}^{0}(X)}} \\
& \cdot \frac{1}{\sqrt{Y_{c}^{0}(X)+\left(1-\sqrt{X_{0}}\right)\left(2-\sqrt{X_{0}}\right)}}(1 \\
& \left.-\sqrt{X_{0}}\right)^{5 / 3} X_{0}^{1 / 12} \frac{1}{\sqrt{2 \pi}}\left[\mathrm{Ai}^{\prime}\left(r_{0}\right)\right]^{-2} \\
& \cdot\left(\int_{r_{0}}^{\infty} e^{\delta_{1} u} \mathrm{Ai}(u) d u\right) \cdot
\end{aligned}
$$

The factors in (492) that precede $\mathscr{L}_{1}$ come from $e^{\rho \Phi(X, Y)}, C_{1}$ (now given by (37)), $e^{\rho^{1 / 3} \Phi_{1}(X, Y)}, L_{1}(X, Y)$, and $L_{0}\left(s_{1}\right)$, except we exclude from $L_{0}(\cdot)$ the factor $\left(s_{1}+W_{0}\right) \sqrt{X_{0}}-s_{1}$, which vanishes if $s_{1} \rightarrow Y_{0}$ and $Y_{0} \rightarrow \sqrt{X_{0}}-X_{0}$. Instead we include an extra factor of $\rho^{-1 / 3}$, and then $\rho^{-1 / 2}=\rho^{-1 / 3} \rho^{-1 / 6}$, where $\rho^{-1 / 6}$ comes from $C_{1}$ in (37). Also, in (492)

$$
\left.Y_{c}^{0}(X) \equiv Y_{c}(X)\right|_{Y_{0}=\sqrt{X_{0}}-X_{0}} .
$$

In region $\mathscr{R}_{3} \cap \mathscr{R}_{4}$ we have $W_{0}+Y_{0}-\sqrt{W_{0}}\left(W_{0}+Y_{0}\right) \sim Y_{c}$ and $Y_{0}+2 W_{0} \sim 2+X_{0}-3 \sqrt{X_{0}}$, and also

$$
\begin{gathered}
\frac{L_{0}\left(s_{1}\right)}{\left(s_{1}+W_{0}\right) \sqrt{X_{0}}-s_{1}} \longrightarrow \\
\frac{1}{2 \pi}\left[\operatorname{Ai}^{\prime}\left(r_{0}\right)\right]^{-2} X_{0}^{-1 / 6}\left(1-\sqrt{X_{0}}\right)^{-4 / 3}, \\
s_{1} \longrightarrow Y_{0} .
\end{gathered}
$$

Thus apart from the factors $\rho^{-1 / 3} \mathscr{L}_{1}(\cdot),(492)$ is just the $\mathscr{D}_{-}$ ray expansion expanded near $Y \rightarrow Y_{c}(X)\left(\right.$ or $s_{1} \rightarrow Y_{0}$ ), 
divided by $\left(s_{1}+W_{0}\right) \sqrt{X_{0}}-s_{1}$. Thus (492) matches to the $\mathscr{D}_{-}$ ray expansion if

$$
\begin{gathered}
\left.\rho^{-1 / 3} \mathscr{L}_{1}\left(\frac{Y_{0}+W_{0}}{Y_{c}+W_{0}} \eta_{* *}\right)\right|_{\eta_{* *} \rightarrow-\infty} \\
\left.\sim\left[\left(s_{1}+W_{0}\right) \sqrt{X_{0}}-s_{1}\right]\right|_{s_{1} \rightarrow Y_{0}} .
\end{gathered}
$$

But the left side of (495), in view of (461) and (462), becomes

$$
\begin{aligned}
& \left(s_{1}+W_{0}\right) \sqrt{X_{0}}-s_{1} \\
& \quad \sim\left(1-\sqrt{X_{0}}\right)^{2} \rho^{-1 / 3}\left(\frac{-\eta_{* *}}{Y_{c}+W_{0}}\right) .
\end{aligned}
$$

Now $Y_{0}+W_{0} \sim 1-\sqrt{X_{0}}$ so that (495) and (496) lead to

$$
\mathscr{L}_{1}(U) \sim\left(1-\sqrt{X_{0}}\right)(-U), \quad U \longrightarrow-\infty .
$$

Next we match (492) to the $\mathscr{D}_{0}$ ray expansion and thus infer the behavior of $\mathscr{L}_{1}(U)$ as $U \rightarrow+\infty$. Now $C_{0} \sim(1-$ $\left.X_{0}-Y_{0}\right) \rho^{-1 / 2} \sim\left(1-\sqrt{X_{0}}\right) \rho^{-1 / 2}$. First evaluating $K(X, Y)$ at $Y_{0}=\sqrt{X_{0}}-X_{0}$ and then letting $Y \rightarrow Y_{c}^{0}$ we get

$$
\begin{gathered}
{\left.\left[\left.K(X, Y)\right|_{Y_{0}=\sqrt{X_{0}}-X_{0}}\right]\right|_{Y \rightarrow Y_{c}^{0}} \sim \sqrt{\frac{2}{\pi} \frac{X_{0}^{1 / 4}\left(1-\sqrt{X_{0}}\right)^{2}}{Y_{c}^{0}(X)}}} \\
\cdot \frac{1}{\sqrt{\sqrt{X_{0}}-X_{0}-Y_{c}^{0}(X)}} \frac{1}{\sqrt{W_{0}+\sqrt{W_{0}}+Y_{c}^{0}(X)}} .
\end{gathered}
$$

The estimate in (478) still holds so if $C_{0} K(X, Y) e^{\rho \Psi(X, Y)}$ is to agree, for $Y \rightarrow Y_{c}$, with the large $\eta_{* *}$ asymptotics of (492), we must have

$$
\begin{aligned}
& {\left[\operatorname{Ai}^{\prime}\left(r_{0}\right)\right]^{-2}\left(\int_{r_{0}}^{\infty} e^{\delta_{1} u} \operatorname{Ai}(u) d u\right)\left(1-\sqrt{X_{0}}\right)^{5 / 3}} \\
& \cdot X_{0}^{1 / 12} \mathscr{L}_{1}\left(\frac{Y_{0}+W_{0}}{Y_{c}+W_{0}} \eta_{* *}\right) \sim 2 X_{0}^{1 / 4}\left(1-\sqrt{X_{0}}\right)^{3} \\
& \cdot \exp \left[r_{0} \frac{\left(1-\sqrt{X_{0}}\right)^{2 / 3}}{X_{0}^{1 / 6}\left(W_{0}+Y_{0}\right)} \eta_{* *}\right. \\
& \left.-\frac{1}{3} \frac{\left(1-\sqrt{X_{0}}\right)^{2}}{\sqrt{X_{0}}}\left(\frac{\eta_{* *}}{W_{0}+Y_{c}}\right)^{3}\right]
\end{aligned}
$$

We thus have the matching condition

$$
\begin{aligned}
& \mathscr{L}_{1}(U) \sim 2 X^{1 / 6}\left(1-\sqrt{X_{0}}\right)^{4 / 3}\left[\operatorname{Ai}^{\prime}\left(r_{0}\right)\right]^{2} \\
& \cdot\left(\int_{r_{0}}^{\infty} e^{\delta_{1} u} \operatorname{Ai}(u) d u\right)^{-1} \\
& \cdot \exp \left[\frac{r_{0} U}{X_{0}^{1 / 6}\left(1-\sqrt{X_{0}}\right)^{1 / 3}}-\frac{U^{3}}{3 \sqrt{X_{0}}\left(1-\sqrt{X_{0}}\right)}\right], \\
& U \longrightarrow+\infty .
\end{aligned}
$$

To determine $\mathscr{L}_{1}(\cdot)$ completely we need to match (492) to the corner layer expansion in (173). Using

$$
\operatorname{Ai}(\nu+\theta) \sim 2^{-1} \pi^{-1 / 2} \nu^{-1 / 4} \exp \left(-\frac{2}{3} \nu^{3 / 2}-\sqrt{\nu} \theta\right)
$$

in the integral we expand (173) for $\nu, T \rightarrow \infty$ with $T-\sqrt{v}=$ $O(1)$. The result contains some exponential factors and some algebraic ones, with the latter being

$$
\begin{gathered}
\rho^{-1 / 3}\left(1-\sqrt{X_{0}}\right)^{4 / 3} X_{0}^{-1 / 3} v^{-1 / 4} \frac{1}{2 \sqrt{\pi}} \frac{1}{2 \pi i} \\
\cdot \int_{-i \infty}^{i \infty} \frac{e^{\theta\left(T-\sqrt{\nu}-\delta_{1}\right)}}{[\operatorname{Ai}(\theta)]^{2}}\left(\int_{\theta}^{\infty} e^{\delta_{1} u} \operatorname{Ai}(u) d u\right) d \theta .
\end{gathered}
$$

Now we evaluate the algebraic part of (492), for $X \rightarrow X_{0}$. From (460), since $Y_{0}+W_{0} \rightarrow 1-\sqrt{X_{0}}=\sqrt{W_{0}}$, we see that $Y_{c}^{0}(X)$ is the same as $Y_{*}(X)$, which is independent of $Y_{0}$. We also have $Y_{*}\left(X_{0}\right)=\sqrt{X_{0}}-X_{0}$ so that (498) becomes singular as $X \rightarrow X_{0}$. We have

$$
\begin{aligned}
& \sqrt{X_{0}}-X_{0}-Y_{*}(X) \\
& =\frac{1}{2}\left[X-X_{0}+\sqrt{X_{0}-X} \sqrt{\left(2-\sqrt{X_{0}}\right)^{2}-X}\right] \\
& \sim\left(1-\sqrt{X_{0}}\right)^{1 / 2} \rho^{-1 / 3} \sqrt{\nu_{1}} .
\end{aligned}
$$

The result in (485) still applies, now simplifying to

$$
\eta_{* *} \sim X_{0}^{1 / 6}\left(1-\sqrt{X_{0}}\right)^{1 / 3}(\sqrt{v}-T) .
$$

With (503) and (504), the algebraic part of (492) becomes

$$
\begin{gathered}
\rho^{-1 / 2} e^{-r_{0}(T-\sqrt{v})} \frac{1}{2 \sqrt{\pi}} \rho^{-1 / 6} \nu^{-1 / 4} X_{0}^{-1 / 4}\left[\operatorname{Ai}^{\prime}\left(r_{0}\right)\right]^{-2} \\
\cdot\left(\int_{r_{0}}^{\infty} e^{\delta_{1} u} \mathrm{Ai}(u) d u\right) \\
\cdot \mathscr{L}_{1}\left(X_{0}^{1 / 6}\left(1-\sqrt{X_{0}}\right)^{1 / 3}(\sqrt{v}-T)\right) .
\end{gathered}
$$

Comparing (502) to (505) determines $\mathscr{L}_{1}(\cdot)$ as

$$
\begin{gathered}
\mathscr{L}_{1}(U)=X_{0}^{1 / 6}\left(1-\sqrt{X_{0}}\right)^{4 / 3}\left[\mathrm{Ai}^{\prime}\left(r_{0}\right)\right]^{2} \\
\cdot\left[\int_{r_{0}}^{\infty} e^{\delta_{1} u} \operatorname{Ai}(u) d u\right]^{-1} \frac{1}{2 \pi i} \\
\cdot \int_{-i \infty}^{i \infty} \frac{e^{-\theta \delta_{1}}}{\operatorname{Ai}^{2}(\theta)}\left(\int_{\theta}^{\infty} e^{\delta_{1} u} \operatorname{Ai}(u) d u\right) \\
\cdot \exp \left[-\frac{U \theta}{X_{0}^{1 / 6}\left(1-\sqrt{X_{0}}\right)^{1 / 3}}\right] d \theta \\
\cdot \exp \left[r_{0} \frac{U}{X_{0}^{1 / 6}\left(1-\sqrt{X_{0}}\right)^{1 / 3}}\right] .
\end{gathered}
$$


We have thus established the result in (194). By asymptotically expanding the contour integral in (506) in the limits $U \rightarrow$ $\pm \infty$ we can easily verify that (497) and (500) are satisfied.

7.3. Transition Layer near $Y=\tilde{Y}(X)$, Region $\mathscr{R}_{1} \cup \mathscr{R}_{2} \cup \mathscr{R}_{3}$. We consider the curve that separates $\mathscr{D}_{0}$ from $\mathscr{D}_{+}$. We use the scaling

$$
Y=\tilde{Y}(X)+\rho^{-1 / 2} \eta_{*}, \quad \eta_{*}=O(1)
$$

and expand the joint distribution as

$$
\begin{gathered}
\pi(k, r) \sim C(\rho) F\left(X, \eta_{*}\right) K_{+}(X, \tilde{Y}) \exp \left[\rho \Psi_{+}(X, \tilde{Y})\right. \\
\left.+\sqrt{\rho} \Psi_{+, Y}(X, \tilde{Y}) \eta_{*}+\frac{1}{2} \Psi_{+, Y Y}(X, \tilde{Y}) \eta_{*}^{2}\right] .
\end{gathered}
$$

The exponential factor in (508) corresponds to the expansions of $\Psi_{+}(X, Y)$ about $Y=\tilde{Y}(X)$. If (508) as $\eta_{*} \rightarrow-\infty$ is to match to the $\mathscr{D}_{+}$ray expansion we must have

$$
F(X,-\infty)=1, \quad \forall 0<X<X_{0} .
$$

We use (508) in the main balance equation (3) and after a lengthy calculation we find that $F\left(X, \eta_{*}\right)$ satisfies the parabolic PDE

$$
\begin{aligned}
& \frac{1}{2}\left[\left(\tilde{Y}^{\prime}\right)^{2}\left(X e^{\Psi_{+, X}}+e^{-\Psi_{+, X}}\right)+\tilde{Y} e^{\Psi_{+, Y}}\right] F_{\eta_{*} \eta_{*}} \\
& \quad+\eta_{*} e^{\Psi_{+, Y}} F_{\eta_{*}}+\left(X e^{\Psi_{+, X}}-e^{-\Psi_{+, X}}\right) F_{X}=0,
\end{aligned}
$$

for $X \in\left(0, X_{0}\right)$ and $\eta_{*} \in(-\infty, \infty)$. In (510), $\Psi_{+}$is understood to be evaluated at $(X, Y)=(X, \tilde{Y}(X))$. We can write the curve $\tilde{Y}(X)$ in parametric form, as

$$
\begin{aligned}
X & =\left(1-\frac{Y_{0}}{X_{0}+Y_{0}} e^{t_{*}}\right)\left[1+\left(X_{0}+Y_{0}-1\right) e^{-t_{*}}\right], \\
\tilde{Y}(X) & =\frac{Y_{0}}{X_{0}+Y_{0}}\left[X_{0}+Y_{0}-1+e^{-t_{*}}\right],
\end{aligned}
$$

with the latter equation corresponding to (178). Since $t_{*}=$ $t_{*}(X)$ we note that

$$
X e^{\Psi_{+, X}(X, \widetilde{Y}(X))}-e^{-\Psi_{+, X}(X, \widetilde{Y}(X))}=-\frac{d X}{d t_{*}}
$$

and define $M_{*}$ from

$$
\begin{aligned}
M_{*}= & {\left[\widetilde{Y}^{\prime}(X)\right]^{2}\left[X e^{\Psi_{+, X}(X, \tilde{Y}(X))}+e^{-\Psi_{+, X}(X, \tilde{Y}(X))}\right] } \\
& +\tilde{Y}(X) e^{\Psi_{+, Y}(X, \tilde{Y}(X))} .
\end{aligned}
$$

We can view $M_{*}$ as being a function of either $X$ or $t_{*}$. In terms of $t_{*}$ we have

$$
\begin{gathered}
M_{*}\left(t_{*}\right)=\frac{Y_{0} e^{-t_{*}}}{X_{0}+Y_{0}} \\
+\left[2+\left(X_{0}+Y_{0}-1\right) e^{-t_{*}}-\frac{Y_{0}}{X_{0}+Y_{0}} e^{t_{*}}\right] \\
+\left[\frac{Y_{0} e^{-t_{*}}}{Y_{0} e^{t_{*}}+\left(X_{0}+Y_{0}\right)\left(X_{0}+Y_{0}-1\right) e^{-t_{*}}}\right]^{2} .
\end{gathered}
$$

Changing variables from $\left(X, \eta_{*}\right)$ to $\left(t_{*}, \eta_{*}\right)$ the PDE in (510) becomes

$$
\begin{aligned}
\frac{1}{2} M_{*} F_{\eta_{*} \eta_{*}}+\frac{\eta_{*}}{1+\left(X_{0}+Y_{0}-1\right) e^{t_{*}}} F_{\eta_{*}}=F_{t_{*}}, & \\
& t_{*}>0
\end{aligned}
$$

and note that $t_{*}=0$ corresponds to $X=X_{0}$, as $\tilde{Y}\left(X_{0}\right)=Y_{0}$.

Next we assume that $F\left(t_{*}, \eta_{*}\right)$ will be a function of a single "similarity" variable, which we call $\star=\eta_{*} a\left(t_{*}\right)$ and with which (515) becomes the ordinary differential equation

$$
\begin{aligned}
& \frac{1}{2} M_{*} a^{2}\left(t_{*}\right) F_{0}^{\prime \prime}(*)+\left[B\left(t_{*}\right)-\frac{a^{\prime}\left(t_{*}\right)}{a\left(t_{*}\right)}\right] \star F_{0}^{\prime}(*) \\
& \quad=0
\end{aligned}
$$

where

$$
\begin{aligned}
F_{0}\left(\eta_{*} a\left(t_{*}\right)\right) & =F\left(t_{*}, \eta_{*}\right), \\
B\left(t_{*}\right) & =\left[1+\left(X_{0}+Y_{0}-1\right) e^{t_{*}}\right]^{-1} .
\end{aligned}
$$

Such a similarity solution is possible if $a\left(t_{*}\right)$ satisfies the nonlinear ODE

$$
B\left(t_{*}\right)-\frac{a^{\prime}\left(t_{*}\right)}{a\left(t_{*}\right)}=\frac{1}{2} M_{*}\left(t_{*}\right) a^{2}\left(t_{*}\right) .
$$

Setting $a\left(t_{*}\right)=\left[b\left(t_{*}\right)\right]^{-1 / 2}$, the Bernoulli equation in (518) becomes the linear equation

$$
b^{\prime}\left(t_{*}\right)+2 B\left(t_{*}\right) b\left(t_{*}\right)=M_{*}\left(t_{*}\right) .
$$

Solving (519) subject to $b(0)=0$ leads to the expression in (184). With (518), (516) becomes

$$
F_{0}^{\prime \prime}(\star)+\star F_{0}^{\prime}(\star)=0
$$

and (509) implies that $F(-\infty)=1$, and thus

$$
F_{0}\left(\frac{\eta_{*}}{\sqrt{b\left(t_{*}\right)}}\right)=\frac{1}{\sqrt{2 \pi}} \int_{\eta_{*} / \sqrt{b\left(t_{*}\right)}}^{\infty} e^{-u^{2} / 2} d u .
$$

We have thus derived (176).

With (521) substituted for $F\left(X, \eta_{*}\right)$ in (508), we can show that as $\eta_{*} \rightarrow+\infty,(508)$ asymptotically matches to the $\mathscr{D}_{0}$ ray expansion. As $\eta_{*} \rightarrow \infty$ from (521) we have

$$
\begin{array}{r}
F_{0}\left(\frac{\eta_{*}}{\sqrt{b\left(t_{*}\right)}}\right) \sim \frac{\sqrt{b\left(t_{*}\right)}}{\sqrt{2 \pi}} \frac{1}{\eta_{*}} \exp \left[-\frac{\eta_{*}^{2}}{2 b\left(t_{*}\right)}\right], \\
\eta_{*} \longrightarrow \infty .
\end{array}
$$

We can easily establish the continuity conditions

$$
\begin{aligned}
\Psi(X, \tilde{Y}(X)) & =\Psi_{+}(X, \tilde{Y}(X))-\Psi_{+}\left(X_{0}, Y_{0}\right), \\
\Psi_{Y}(X, \tilde{Y}(X)) & =\Psi_{+, Y}(X, \tilde{Y}(X))
\end{aligned}
$$


and, after a lengthy calculation, show that

$$
\Psi_{+, Y Y}(X, \tilde{Y}(X))=\frac{1}{b\left(t_{*}\right)}+\Psi_{Y Y}(X, \tilde{Y}(X)) \text {. }
$$

Thus expanding the $\mathscr{D}_{0}$ ray expansion, $C_{0} K(X, Y) e^{\rho \Psi(X, Y)}$, as $Y \downarrow \tilde{Y}(X)$ and comparing this to the large $\eta_{*}$ expansion of (508), using (523) and (524), lead to

$$
\begin{aligned}
& C_{0} K(X, Y) \\
& \sim C K_{+}(X, \tilde{Y}(X)) \frac{\sqrt{b\left(t_{*}\right)}}{\sqrt{2 \pi}} \frac{e^{\rho \Psi_{+}\left(X_{0}, Y_{0}\right)}}{\sqrt{\rho[Y-\tilde{Y}(X)]}}, \\
& Y \longrightarrow \tilde{Y}(X) .
\end{aligned}
$$

But from Proposition 2 we see that, for $\mathscr{R}_{1} \cup \mathscr{R}_{2} \cup \mathscr{R}_{3}$,

$$
\begin{aligned}
& \frac{C}{C_{0}} \sim \sqrt{2 \pi \rho} \sqrt{X_{0}+Y_{0}} \exp \left\{\rho \left[1-X_{0}-Y_{0}\right.\right. \\
& \left.\left.\quad+\left(X_{0}+Y_{0}\right) \log \left(X_{0}+Y_{0}\right)\right]\right\}, \\
& \Psi_{+}\left(X_{0}, Y_{0}\right)=-1+X_{0}+Y_{0}-\left(X_{0}+Y_{0}\right) \log \left(X_{0}\right. \\
& \left.\quad+Y_{0}\right) .
\end{aligned}
$$

The curve $Y=\tilde{Y}(X)$ corresponds to the ray $A=Y_{0} /\left(X_{0}+Y_{0}\right)$, and then $B=1-X_{0}-Y_{0}$. From (70) we see that $K$ becomes singular as $Y \rightarrow \tilde{Y}(X)$, due to the factor $\left[Y_{0}-A\left(X_{0}+Y_{0}\right)\right]^{-1}$. This singularity precisely matches that in the right-hand side of (525), and yet another lengthy calculation shows that

$$
\begin{aligned}
& \lim _{Y \rightarrow \widetilde{Y}(X)}\{[Y-\tilde{Y}(X)] K(X, Y)\} \\
& \quad=\sqrt{X_{0}+Y_{0}} \sqrt{b\left(t_{*}\right)} K_{+}(X, \tilde{Y}(X)),
\end{aligned}
$$

which establishes the matching.

We note that the PDE (515) contains many solutions other than the similarity solution. An initial condition as $t_{*} \rightarrow$ 0 can be obtained by asymptotically matching (508) to the corner layer valid on the $(n, p)$ scale. This will determine the solution to (515) uniquely, but the matching will again lead to the conclusion that $F$ is given by (521). Below we only briefly verify that the matching holds. Near the corner the curve $Y=\tilde{Y}(X)$ can be approximated by the straight line $Y-Y_{0}=\tilde{Y}^{\prime}\left(X_{0}\right)\left(X-X_{0}\right)$, which corresponds to

$$
\frac{Y_{0}-Y}{X_{0}-X}=\frac{p}{n}=\frac{Y_{0}}{\left(X_{0}+Y_{0}\right)^{2}-X_{0}}=\frac{1}{\theta_{1}},
$$

where $\theta_{1}$ was defined in Proposition 20. Thus we must show that (508) for $X \rightarrow X_{0}$ is the same as (251). The exponential parts agree automatically and $C(\rho) \sim \rho^{-1 / 2} Q(0,0)$, so we must only show that, for $X \rightarrow X_{0}$,

$$
\begin{gathered}
K_{+}(X, \tilde{Y}) \sqrt{X_{0}+Y_{0}} \int_{\eta_{*} / \sqrt{b\left(t_{*}\right)}}^{\infty} e^{-u^{2} / 2} d u \\
\sim \frac{\left(X_{0}+Y_{0}\right)^{2}-X_{0}}{\left(X_{0}+Y_{0}\right)^{2}} \frac{1}{\sqrt{2 \pi}} \int_{-\infty}^{\eta} e^{-u^{2} / 2} d u .
\end{gathered}
$$

But

$$
\begin{aligned}
K_{+} & \left(X_{0}, Y_{0}\right) \\
& =(2 \pi)^{-1 / 2}\left[\left(X_{0}+Y_{0}\right)^{2}-X_{0}\right]\left(X_{0}+Y_{0}\right)^{-5 / 2}, \\
\eta_{*} & =\sqrt{\rho}[Y-\tilde{Y}(X)]=\sqrt{\rho}\left[Y-Y_{0}+Y_{0}-\tilde{Y}(X)\right] \\
& =-\frac{p}{\sqrt{\rho}}+\sqrt{\rho}\left[Y_{0}-\tilde{Y}(X)\right] \\
& \sim \frac{1}{\sqrt{\rho}}\left[-p+\frac{Y_{0}}{\left(X_{0}+Y_{0}\right)^{2}-X_{0}} n\right] .
\end{aligned}
$$

From (184)-(187) we obtain, as $X \rightarrow X_{0}$ or $t_{*} \rightarrow 0$,

$$
\begin{aligned}
& b\left(t_{*}\right) \\
& \sim \frac{Y_{0}\left(X_{0}+Y_{0}\right)\left[\left(X_{0}+Y_{0}\right)^{3}+\left(X_{0}+Y_{0}\right)\left(Y_{0}-2 X_{0}\right)+X_{0}\right]}{\left[\left(X_{0}+Y_{0}\right)^{2}-X_{0}\right]^{3}} \frac{n}{\rho}
\end{aligned}
$$

since $n / p \sim \theta_{1}$, as $t_{*} \rightarrow 0 \eta_{*} / \sqrt{b\left(t_{*}\right)}$ approaches $-\eta$ in (252), which verifies (529). This completes the analysis of the transition from $\mathscr{D}_{+}$to $\mathscr{D}_{0}$.

\section{Numerical Studies and Discussion}

Next we show that our asymptotic results can also be used to accurately estimate the probabilities $\pi(k, r)$ in ranges where there is little mass. We will consider $\pi(0, R)$ which is the (very unlikely) situation where no primary spaces are occupied but all of the secondary spaces are full. To estimate $\pi(0, R)$ we must use the expansion that is valid for $k=O(1)$ and $p=R-$ $r=O(1)$, and this corresponds to (130). Thus we set $k=p=0$ in (130) and note that $Q(0,0)$ has the different expansions in Proposition 1 , according to regions in parameter space. In Table 1 we take $X_{0}=2, Y_{0}=1$ and use the expression for $Q(0,0)$ that holds in parameter region $\mathscr{R}_{1}$. We see that the exact and asymptotic results agree to three decimal places even if $\rho$ is as small as 2 . In Table 2 we have $X_{0}=0.8$ and $Y_{0}=0.4$, and $Q(0,0)$ is again computed from (30). Now the agreement is not quite as good as in Table 1, and we typically have errors of about $10 \%$. This is probably due to the fact that numerically $X_{0}+Y_{0}=1.2$, which exceeds one only slightly, so it may be preferable to use (29) to approximate $Q(0,0)$. In Tables 3 and 4 we take, respectively, $X_{0}=0.4, Y_{0}=0.4$ and $X_{0}=0.4, Y_{0}=0.2$. Table 3 corresponds to region $\mathscr{R}_{3}$ and Table 4 to region $\mathscr{R}_{4}$. The agreement is certainly better for region $\mathscr{R}_{4}$, as again the sum $X_{0}+Y_{0}$ is further away from the critical value of one. Here we used $Q(0,0) \sim 1-X_{0}-Y_{0}$ for region(s) $\mathscr{R}_{3} \cup \mathscr{R}_{4}$.

Tables $1-4$ show that the very small values of $\pi(0, R)$ are well predicted by the asymptotic formula(s).

To summarize, we have done a rather thorough asymptotic analysis for this storage allocation model. We have shown that as long as $X_{0}+Y_{0}>1(m+R>\rho)$ the effects of the finiteness of the secondary storage capacity occur only for $(X, Y) \in \mathscr{D}_{0}$ and for those state space regions that border $\mathscr{D}_{0}$. However, for $X_{0}+Y_{0}<1(m+R<\rho)$ the entire state 
TABLE 1: $\pi(0, R)$ for region $\mathscr{R}_{1}, X_{0}=2, Y_{0}=1$.

\begin{tabular}{lcc}
\hline$\rho$ & Exact & Asymptotic \\
\hline 2 & $5.41\left(10^{-4}\right)$ & $5.41\left(10^{-4}\right)$ \\
4 & $2.54\left(10^{-7}\right)$ & $2.54\left(10^{-7}\right)$ \\
6 & $1.19\left(10^{-10}\right)$ & $1.19\left(10^{-10}\right)$ \\
8 & $5.58\left(10^{-14}\right)$ & $5.58\left(10^{-14}\right)$ \\
10 & $2.62\left(10^{-17}\right)$ & $2.62\left(10^{-17}\right)$ \\
\hline
\end{tabular}

TABLE 2: $\pi(0, R)$ for region $\mathscr{R}_{2}, X_{0}=0.8, Y_{0}=0.4$.

\begin{tabular}{lcc}
\hline$\rho$ & Exact & Asymptotic \\
\hline 5 & $1.47\left(10^{-3}\right)$ & $1.13\left(10^{-3}\right)$ \\
10 & $1.70\left(10^{-6}\right)$ & $1.35\left(10^{-6}\right)$ \\
15 & $1.96\left(10^{-9}\right)$ & $1.61\left(10^{-9}\right)$ \\
20 & $2.27\left(10^{-12}\right)$ & $1.92\left(10^{-12}\right)$ \\
25 & $2.65\left(10^{-15}\right)$ & $2.29\left(10^{-15}\right)$ \\
30 & $3.09\left(10^{-18}\right)$ & $2.73\left(10^{-18}\right)$ \\
35 & $3.63\left(10^{-21}\right)$ & $3.25\left(10^{-21}\right)$ \\
\hline
\end{tabular}

TABLE 3: $\pi(0, R)$ for region $\mathscr{R}_{3}, X_{0}=0.4, Y_{0}=0.4$.

\begin{tabular}{lcc}
\hline$\rho$ & Exact & Asymptotic \\
\hline 10 & $1.86\left(10^{-4}\right)$ & $1.08\left(10^{-4}\right)$ \\
20 & $2.29\left(10^{-8}\right)$ & $1.56\left(10^{-8}\right)$ \\
30 & $2.64\left(10^{-12}\right)$ & $1.94\left(10^{-12}\right)$ \\
40 & $2.96\left(10^{-16}\right)$ & $2.28\left(10^{-16}\right)$ \\
50 & $3.25\left(10^{-20}\right)$ & $2.59\left(10^{-20}\right)$ \\
60 & $3.53\left(10^{-24}\right)$ & $2.89\left(10^{-24}\right)$ \\
70 & $3.81\left(10^{-28}\right)$ & $3.18\left(10^{-28}\right)$ \\
\hline
\end{tabular}

TABLE $4: \pi(0, R)$ for region $\mathscr{R}_{4}, X_{0}=0.4, Y_{0}=0.2$.

\begin{tabular}{lcc}
\hline$\rho$ & Exact & Asymptotic \\
\hline 10 & $5.13\left(10^{-4}\right)$ & $4.17\left(10^{-4}\right)$ \\
20 & $1.33\left(10^{-7}\right)$ & $1.17\left(10^{-7}\right)$ \\
30 & $3.12\left(10^{-11}\right)$ & $2.83\left(10^{-11}\right)$ \\
40 & $6.99\left(10^{-15}\right)$ & $6.48\left(10^{-15}\right)$ \\
50 & $1.53\left(10^{-18}\right)$ & $1.43\left(10^{-18}\right)$ \\
60 & $3.29\left(10^{-22}\right)$ & $3.11\left(10^{-22}\right)$ \\
70 & $6.98\left(10^{-26}\right)$ & $6.66\left(10^{-26}\right)$ \\
\hline
\end{tabular}

space is affected by the finite capacity, as then there are not enough storage spaces to satisfy the demand for them. For $X_{0}+Y_{0}<1$ it proves useful to consider the numbers, $m-N_{1}$ and $R-N_{2}$, of empty primary and secondary spaces, and then the steady state distribution $\pi(k, r)=\pi(m-n, R-p)$ has for $\rho \rightarrow \infty$ the limiting form in (50). Even though the marginal distributions of $N_{1}$ and the sum $N_{1}+N_{2}$ are particularly simple, the joint distribution of $\left(N_{1}, N_{2}\right)$ is quite complicated, as the analysis involves many different ranges of the parameter space (cf. Figure 2) and the state space (cf. Figures 3-6). In some ranges special functions such as Airy and parabolic cylinder functions play a key role.

\section{Competing Interests}

The authors declare that they have no competing interests.

\section{Acknowledgments}

This work is partially supported by a Faculty Development Grant from Columbia College Chicago.

\section{References}

[1] D. E. Knuth, Fundamental Algorithms, vol. 1, Addison-Wesley, Reading, Mass, USA, 3rd edition, 1997.

[2] S. Xie and C. Knessl, "On the transient behavior of the Erlang loss model: heavy usage asymptotics," SIAM Journal on Applied Mathematics, vol. 53, no. 2, pp. 555-599, 1993.

[3] L. Kosten, "Uber sperrungswahrscheinlichkeiten bei staffelschltungen," ElectraNachr.-Tech, vol. 14, pp. 5-12, 1937.

[4] D. Aldous, "Some interesting processes arising as heavy traffic limits in an $M / M / \infty$ storage process," Stochastic Processes and Their Applications, vol. 22, pp. 291-313, 1986.

[5] E. G. Coffman Jr., T. T. Kadota, and L. A. Shepp, "A stochastic model of fragmentation in dynamic storage allocation," SIAM Journal on Computing, vol. 14, no. 2, pp. 416-425, 1985.

[6] G. F. Newell, The $M / M / \infty$ Service System with Ranked Servers in Heavy Traffic, Springer, New York, NY, USA, 1984.

[7] J. Preater, "A perpetuity and the $M / M / \infty$ ranked server system," Journal of Applied Probability, vol. 34, no. 2, pp. 508513, 1997.

[8] E. Sohn and C. Knessl, "A simple direct solution to a storage allocation model," Applied Mathematics Letters, vol. 21, no. 2, pp. 172-175, 2008.

[9] C. Knessl, "Asymptotic expansions for a stochastic model of queue storage," The Annals of Applied Probability, vol. 10, no. 2, pp. 592-615, 2000.

[10] C. Knessl, "Geometrical optics and models of computer memory fragmentation," Studies in Applied Mathematics, vol. 111, no. 2, pp. 185-238, 2003.

[11] C. Knessl, "Some asymptotic results for the $M / M / \infty$ queue with ranked servers," Queueing Systems, vol. 47, no. 3, pp. 201250, 2004.

[12] E. Sohn and C. Knessl, "The distribution of wasted spaces in the $M / M / \infty$ queue with ranked servers," Advances in Applied Probability, vol. 40, pp. 835-855, 2008.

[13] E. Sohn and C. Knessl, "On a storage allocationmodel with finite capacity," European Journal of Applied Mathematics, 2016.

[14] R. Courant and D. Hilbert, Methods of Mathematical Physics, Partial Differential Equations, vol. 2, Wiley-Interscience, New York, NY, USA, 1989.

[15] N. Bleistein and R. A. Handelsman, Asymptotic Expansions of Integrals, Dover, New York, NY, USA, 2nd edition, 1986.

[16] C. M. Bender and S. A. Orszag, Advanced Mathematical Methods for Scientists and Engineers, McGraw-Hill, New York, NY, USA, 1978.

[17] R. Wong, Asymptotic Approximation of Integrals, SIAM, Philadelphia, Pa, USA, 2001.

[18] P. Flajolet and R. Sedgewick, Analytic Combinatorics, Cambridge University Press, Cambridge, UK, 2009.

[19] W. Szpankowski, Average Case Analysis of Algorithms on Sequences, Wiley-Interscience, New York, NY, USA, 2001. 
[20] C. Knessl and Y. Yang, "Analysis of a Brownian particle moving in a time-dependent drift field," Asymptotic Analysis, vol. 27, no. 3-4, pp. 281-319, 2001.

[21] C. Knessl, "On two-dimensional convection-diffusion past a circle," SIAM Journal on Applied Mathematics, vol. 62, no. 1, pp. 310-335, 2001. 


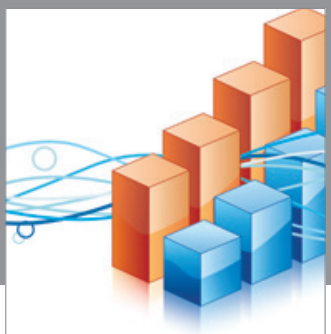

Advances in

Operations Research

vatem alat4

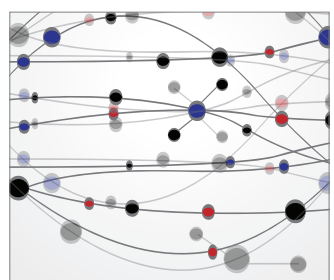

\section{The Scientific} World Journal
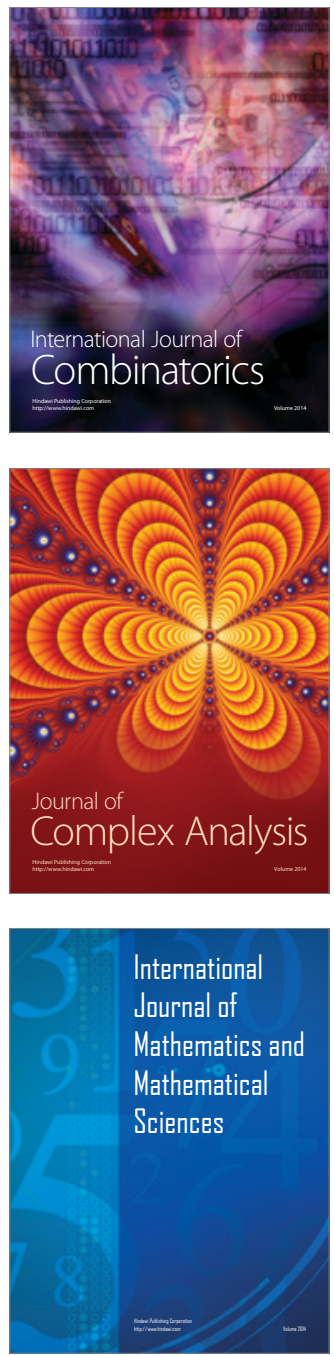
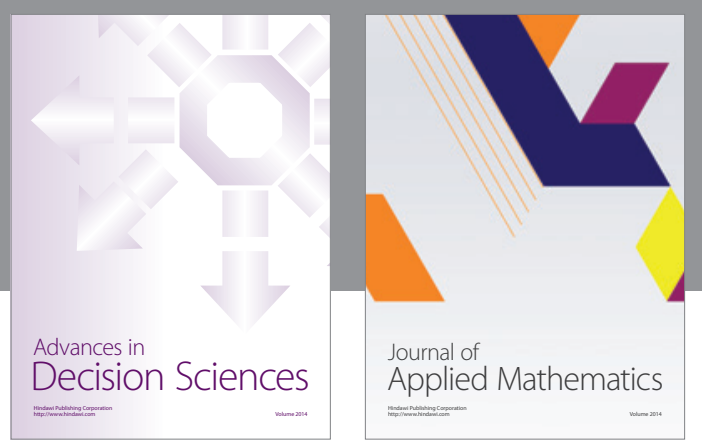

Algebra

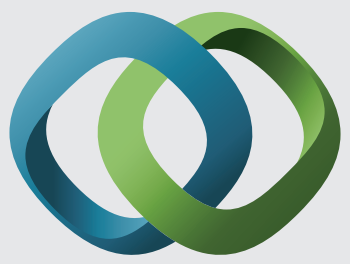

\section{Hindawi}

Submit your manuscripts at

http://www.hindawi.com
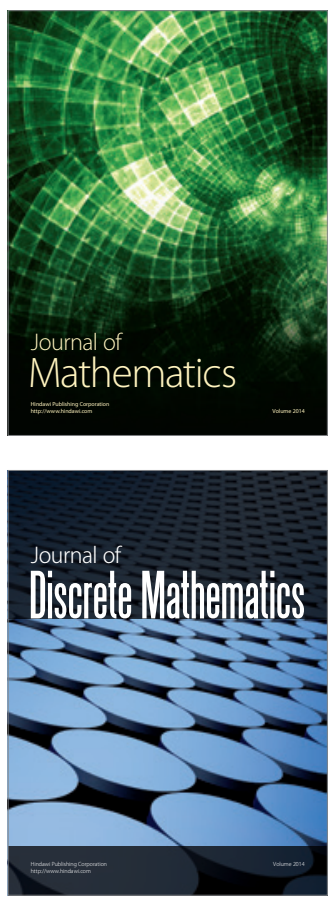

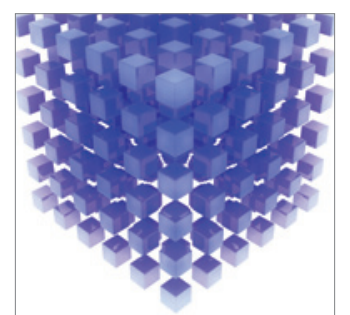

Mathematical Problems in Engineering
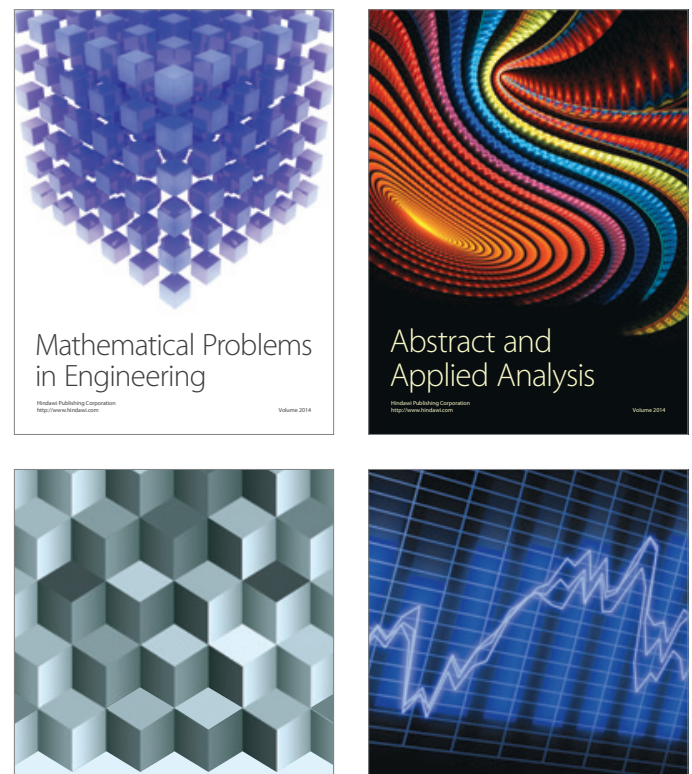

Journal of

Function Spaces

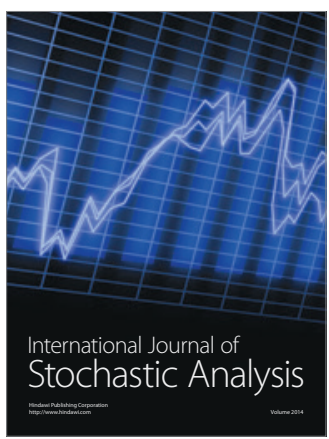

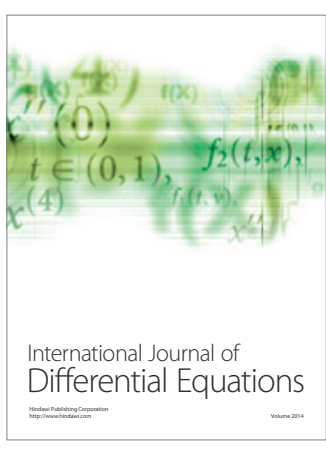
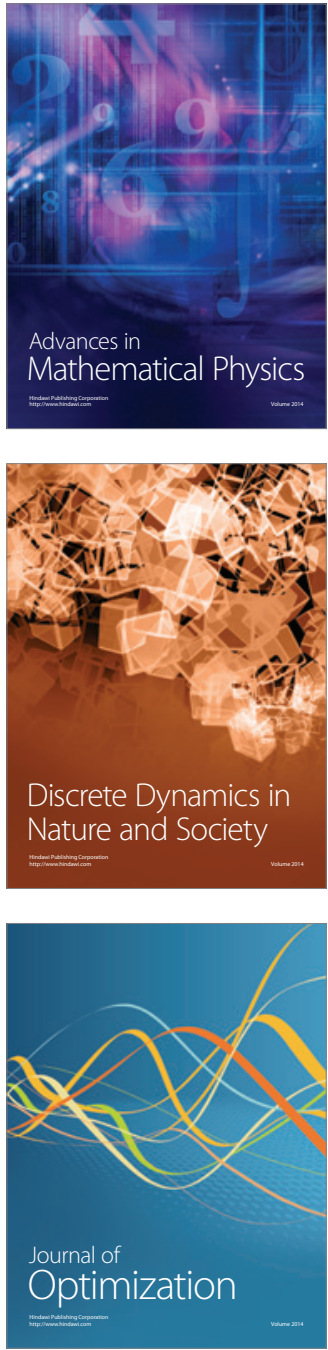